\title{
A dearomatizing, thionium ion cyclization for the synthesis of functionalized, azaspirocyclic cyclohexadienones
}

\author{
Caroline Ovens ${ }^{+}$, Nathaniel G. Martin ${ }^{\S}$ and David J. Procter ${ }^{+*}$ \\ ${ }^{+}$The University of Manchester, Oxford Road, Manchester, M13 9PL, UK \\ ${ }^{\S}$ AstraZeneca, Mereside, Alderley Park, Macclesfield, Cheshire, SK10 4TG, UK \\ *E-mail: david.j.procter@manchester.ac.uk \\ Fax: (+0044) 01612754939
}

S2-S3 General experimental

S3-S43 Experimental

S44-S54 NMR spectra for $\alpha$-hydroxyamides

S55-S76 NMR spectra for cyclisations

S77-S85 NMR spectra for spirocycle modifications

S86 X-ray crystal structures for $\mathbf{1 0}$ and $\mathbf{3 1}$ 


\section{General Experimental}

All reactions were carried out under an inert nitrogen atmosphere unless otherwise stated. Glassware for inert atmosphere reactions was oven-dried and cooled under a flow of nitrogen. Tetrahydrofuran was distilled over sodium wire and benzophenone; dichloromethane was distilled over calcium hydride. Triethylamine was distilled from calcium hydride and stored over $\mathrm{KOH}$. DMSO was distilled over calcium hydride, under reduced pressure, and stored over molecular sieves. All other solvents and reagents were purchased from commercial sources and used as supplied.

NMR spectra were recorded on one of the following spectrometers: Varian 300 MHz, Varian $400 \mathrm{MHz}$, Bruker Avance $400 \mathrm{MHz}$, Bruker DPX400 MHz, Bruker DPX500 MHz, Bruker $500 \mathrm{MHz}$. All chemical shift values are reported in ppm, with coupling constants in Hz. The notation of signals is: -

$\delta_{\mathrm{H}}$ chemical shift in ppm (number of protons, multiplicity, $J$ value(s), proton assignment) $\delta_{\mathrm{C}}$ chemical shift in ppm (carbon assignment)

If assignment is ambiguous, for example in the case of overlapping aromatic signals, a range of shifts is reported.

NMR assignments were perfomed with the aid of COSY, HMQC, DEPT 135 and DEPT 90 experiments.

Routine TLC analysis was carried out on aluminium sheets coated with Merck silica gel $60 \mathrm{~F} 254,0.2 \mathrm{~mm}$ thickness. Solvent systems were either $40-60^{\circ}$ petroleum ether/ethyl acetate mixtures or methanol/dichloromethane mixtures. Plates were viewed with a 254 $\mathrm{nm}$ ultraviolet lamp and dipped in aqueous potassium permanganate or DNP.

Flash column chromatography was carried out on Fisher Scientific $35-70 \mu, 60 \mathrm{~A}$ silica gel. Fluorous solid phase extraction (FSPE) was performed on FluoroFlash ${ }^{\circledR}$ silica gel. 
Low-resolution mass spectra were recorded on a Fissions VG Trio 2000 quadrupole mass spectrometer or a Thermo LTQ FT spectrometer. Spectra were obtained using electron impact ionisation (EI) and chemical ionisation (CI) techniques, or positive and/or negative electrospray ionisation.

High-resolution mass spectra were recorded on a Thermo Finnigan MAT 95XP mass spectrometer.

GC/MS spectra were recorded on a Waters GCT Premier spectrometer or a Shimadzu GCMS QP2010 spectrometer.

Melting points were measured on a Sanyo Gallenkamp variable heater apparatus and are uncorrected.

IR spectra were recorded on an ATI Mattson Genesis Series FTIR spectrometer as evaporated films (from dichloromethane) or neat, using sodium chloride windows.

\section{Experimental}

General procedure A: To the secondary benzylamine (1 eq) in $\mathrm{CH}_{2} \mathrm{Cl}_{2}(5 \mathrm{ml} / \mathrm{mmol})$ was added acetoxyacetic acid (1.2 eq), 1-hydroxybenzotriazole hydrate (0.2 eq) and EDCI (1.2 eq) and the mixture stirred at room temperature for 16-27 hours. The reaction mixture was washed with $1 \mathrm{M} \mathrm{HCl}(\times 3)$ and the organic layer dried $\left(\mathrm{Na}_{2} \mathrm{SO}_{4}\right)$ and concentrated in vacuo to give the crude amide. To the acetoxyacetamide (1 eq) in 2:1 $\mathrm{MeOH} / \mathrm{H}_{2} \mathrm{O}(6 \mathrm{ml} / \mathrm{mmol})$ was added $\mathrm{K}_{2} \mathrm{CO}_{3}$ (4 eq) and the resulting mixture stirred at room temperature for 2-27 hours. $\mathrm{MeOH}$ was removed in vacuo, the residue taken up in $\mathrm{H}_{2} \mathrm{O}$ and extracted with ethyl acetate $(\times 3)$. The combined organic layers were dried $\left(\mathrm{Na}_{2} \mathrm{SO}_{4}\right)$ and concentrated in vacuo to afford $\alpha$-hydroxyamide.

General procedure B: To oxalyl chloride (1.1 eq) in $\mathrm{CH}_{2} \mathrm{Cl}_{2}(4 \mathrm{ml} / \mathrm{mmol})$ was added DMSO (2 eq) in $\mathrm{CH}_{2} \mathrm{Cl}_{2}(1 \mathrm{ml} / \mathrm{mmol})$ via cannula at $-78{ }^{\circ} \mathrm{C}$. The mixture was stirred for 30 minutes at $-78{ }^{\circ} \mathrm{C}$. $\alpha$-Hydroxyamide $(1 \mathrm{eq})$ in $\mathrm{CH}_{2} \mathrm{Cl}_{2}(4 \mathrm{ml} / \mathrm{mmol})$ was added via cannula at $-78{ }^{\circ} \mathrm{C}$ and the mixture was stirred at this temperature for 1 hour. 
Triethylamine (5 eq) was added and the mixture allowed to warm to room temperature. The resulting yellow solution was stirred at room temperature for 1-26 hours. The organic layer was washed with aqueous saturated $\mathrm{NaHCO}_{3}(\times 3)$, dried $\left(\mathrm{Na}_{2} \mathrm{SO}_{4}\right)$ and concentrated in vacuo to yield crude glyoxamide, usually as a yellow foam. To the crude glyoxamide (1 eq) in $\mathrm{CH}_{2} \mathrm{Cl}_{2}(12 \mathrm{ml} / \mathrm{mmol})$ was added $1 \mathrm{H}, 1 \mathrm{H}, 2 \mathrm{H}, 2 \mathrm{H}$-perfluorodecane-1thiol $(0.7 \mathrm{eq})$ or a non-fluorous thiol $(1 \mathrm{eq})$ and the mixture was stirred at room temperature for 12-40 hours. Trifluoroacetic anhydride ( 9 eq) was added and the mixture was stirred at room temperature for 1 hour before the addition of $\mathrm{BF}_{3} \cdot \mathrm{OEt}_{2}(5 \mathrm{eq})$. After 3-6 hours stirring at room temperature, during which time the solution adopted an intense colour, aqueous saturated $\mathrm{NaHCO}_{3}$ was added resulting in vigorous effervescence. The aqueous layer was extracted with $\mathrm{CH}_{2} \mathrm{Cl}_{2}(\times 3)$. The combined organic layers were washed with aqueous saturated $\mathrm{NaHCO}_{3}(\times 3)$, dried $\left(\mathrm{Na}_{2} \mathrm{SO}_{4}\right)$ and concentrated in vacuo to yield crude spirocycle.

Where indicated aqueous saturated $\mathrm{NaHCO}_{3}$ solution was exchanged for $1 \mathrm{M} \mathrm{NaOH}$.

\section{2-Hydroxy- $N$-iso-propyl- $N$-(2,4,6-trimethoxybenzyl)acetamide 39}

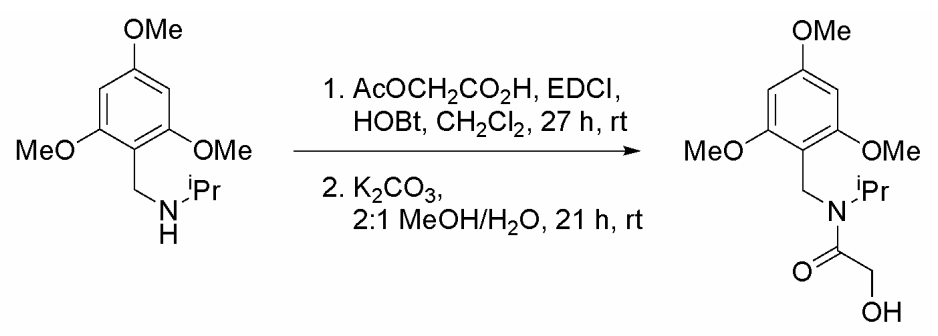

Following general procedure A: $N$-iso-Propyl- $N-2,4,6$-trimethoxybenzylamine $(11.2 \mathrm{~g}$, $46.9 \mathrm{mmol}, 1 \mathrm{eq}$ ) was stirred with acetoxyacetic acid (6.64 g, $56.2 \mathrm{mmol}, 1.2 \mathrm{eq}), \mathrm{HOBt}$ $(1.27 \mathrm{~g}, 9.37 \mathrm{mmol}, 0.2 \mathrm{eq})$ and EDCI $(10.8 \mathrm{~g}, 56.2 \mathrm{mmol}, 1.2 \mathrm{eq})$. The resulting crude amide (14.3 g, $42.1 \mathrm{mmol}, 1 \mathrm{eq})$ was stirred with $\mathrm{K}_{2} \mathrm{CO}_{3}(23.3 \mathrm{~g}, 168 \mathrm{mmol}, 4 \mathrm{eq})$. Recrystallisation (EtOH) of the white solid afforded 2-hydroxy- $N$-iso-propyl- $N$-(2,4,6trimethoxybenzyl)acetamide 39 as a white crystalline solid (9.76 g, $32.9 \mathrm{mmol}, 70 \%$ over two steps). 
$\delta_{\mathrm{H}}\left(500 \mathrm{MHz}, \mathrm{CDCl}_{3}\right) 1.11\left(6 \mathrm{H}, \mathrm{d}, J=6.9 \mathrm{~Hz}, 2 \times \mathrm{C}_{3} \mathrm{CH}\right), 3.80\left(6 \mathrm{H}, \mathrm{s}, 2 \times \underline{\mathrm{C}}_{3} \mathrm{O}\right), 3.82$ $\left(3 \mathrm{H}, \mathrm{s}, \underline{\mathrm{C}}_{3} \mathrm{O}\right), 3.88\left(1 \mathrm{H}\right.$, septet, $\left.J=6.9 \mathrm{~Hz}, \mathrm{C} \underline{\mathrm{H}}\left(\mathrm{CH}_{3}\right)_{2}\right), 3.92\left(1 \mathrm{H}, \mathrm{t}, J=4.2 \mathrm{~Hz}, \mathrm{CH}_{2} \mathrm{OH}\right)$, $4.24\left(2 \mathrm{H}, \mathrm{s}, \mathrm{C}_{2} \mathrm{~N}\right), 4.34\left(2 \mathrm{H}, \mathrm{d}, J=4.2 \mathrm{~Hz}, \underline{\mathrm{C}}_{2} \mathrm{OH}\right), 6.11(2 \mathrm{H}, \mathrm{s}, 2 \times \mathrm{Ar} \underline{\mathrm{H}}) ; \delta_{\mathrm{C}}(125$ $\left.\mathrm{MHz}, \mathrm{CDCl}_{3}\right) 19.5\left(2 \times \underline{\mathrm{CH}}_{3} \mathrm{CH}\right), 37.3\left(\underline{\mathrm{CH}}_{2} \mathrm{~N}\right), 49.3\left(\underline{\mathrm{CH}}\left(\mathrm{CH}_{3}\right)_{2}\right), 55.3\left(\underline{\mathrm{CH}}_{3} \mathrm{O}\right), 55.6(2 \times$ $\left.\underline{\mathrm{CH}}_{3} \mathrm{O}\right), 60.6\left(\underline{\mathrm{C}} \mathrm{H}_{2} \mathrm{O}\right), 90.4(2 \times \mathrm{Ar} \underline{\mathrm{CH}}), 104.7(\mathrm{Ar} \underline{\mathrm{C}}), 159.7\left(2 \times \underline{\mathrm{C}}\left(\mathrm{OCH}_{3}\right)\right), 161.3$ $\left(\underline{\mathrm{C}}\left(\mathrm{OCH}_{3}\right)\right), 171.5(\mathrm{NCO}) ; v_{\max }\left(\mathrm{CH}_{2} \mathrm{Cl}_{2}\right.$ evaporated film/ $\left./ \mathrm{cm}^{-1}\right) 3391$ (broad, $\left.\mathrm{OH}\right), 2966$ and $2930(\mathrm{CH}), 1634$ (amide $\mathrm{CO}), 1609(\mathrm{C}=\mathrm{C}) ; \mathrm{m} / \mathrm{z}\left(\mathrm{EI}^{+}\right) 298\left(\mathrm{MH}^{+}, 12 \%\right), 254(27 \%)$, 181 (100\%); m/z (CI+) $298\left(\mathrm{MH}^{+}, 94 \%\right), 254$ (62\%), 181 (100\%); $\mathrm{C}_{15} \mathrm{H}_{24} \mathrm{NO}_{5}\left(\mathrm{MH}^{+}\right)$ found 298.1648, expected 298.1649; $\mathrm{mp}(\mathrm{EtOH}) 117.7-119.1^{\circ} \mathrm{C}$.

\section{2-Hydroxy- $N$-(2,4,6-trimethoxybenzyl)- $N$-(phenylsulfonylethyl)acetamide 40}
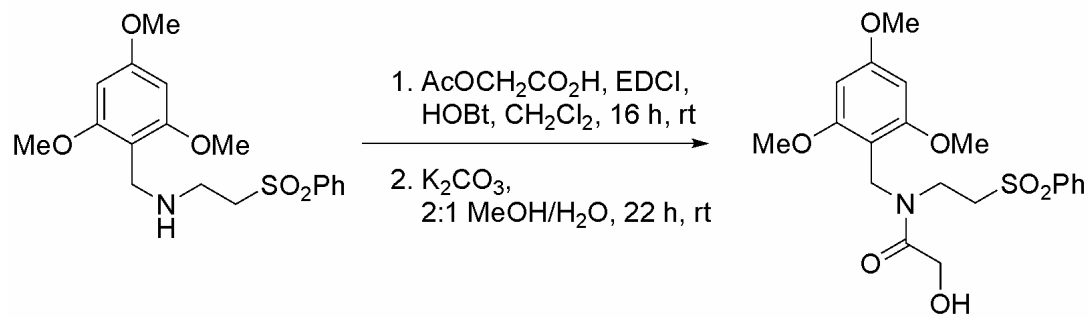

Following general procedure A: $N$-(2,4,6-Trimethoxybenzyl)- $N$-(phenylsulfonylethyl) amine $(0.93 \mathrm{~g}, 2.55 \mathrm{mmol}, 1 \mathrm{eq})$ was stirred with acetoxyacetic acid $(0.95 \mathrm{~g}, 3.06 \mathrm{mmol}$, $3.2 \mathrm{eq}), \mathrm{HOBt}(69 \mathrm{mg}, 0.51 \mathrm{mmol}, 0.2 \mathrm{eq})$ and EDCI (0.59 g, $30.6 \mathrm{mmol}, 1.2 \mathrm{eq})$. The resulting crude amide $(1.15 \mathrm{~g}, 2.47 \mathrm{mmol}, 1 \mathrm{eq})$ was stirred with $\mathrm{K}_{2} \mathrm{CO}_{3}(1.37 \mathrm{~g}, 9.87$ mmol, 4 eq). Recrystallisation (EtOH) afforded 2-hydroxy- $N$-(2,4,6-trimethoxybenzyl)$N$-(phenylsulfonylethyl)acetamide $\mathbf{4 0}$ as a white crystalline solid $(0.76 \mathrm{~g}, 1.79 \mathrm{mmol}$, $72 \%$ over two steps).

$\delta_{\mathrm{H}}\left(500 \mathrm{MHz}, \mathrm{CDCl}_{3}\right) 3.08-3.15\left(2 \mathrm{H}, \mathrm{m}, \mathrm{C}_{2} \mathrm{CH}_{2} \mathrm{SO}_{2} \mathrm{Ph}\right), 3.41(1 \mathrm{H}, \mathrm{t}, J=4.1 \mathrm{~Hz}$, $\left.\mathrm{CH}_{2} \mathrm{OH}\right)$ 3.49-3.55 (2H, m, $\left.\mathrm{C}_{2} \mathrm{CH}_{2} \mathrm{SO}_{2} \mathrm{Ph}\right), 3.73\left(6 \mathrm{H}, \mathrm{s}, 2 \times \mathrm{C}_{3} \mathrm{O}\right), 3.77\left(3 \mathrm{H}, \mathrm{s}, \mathrm{C}_{3} \mathrm{O}\right)$, $4.16\left(2 \mathrm{H}, \mathrm{s}, \mathrm{C}_{2} \mathrm{~N}\right), 4.26\left(2 \mathrm{H}, \mathrm{d}, J=4.1 \mathrm{~Hz}, \underline{\mathrm{C}}_{2} \mathrm{OH}\right), 6.04(2 \mathrm{H}, \mathrm{s}, 2 \times \mathrm{Ar} \underline{\mathrm{H}}) ; 7.46-7.51$ $(2 \mathrm{H}, \mathrm{m}, 2 \times \mathrm{Ar} \underline{\mathrm{H}}), 7.55-7.61(1 \mathrm{H}, \mathrm{m}, \mathrm{Ar} \underline{\mathrm{H}}), 7.75-7.78(2 \mathrm{H}, \mathrm{m}, 2 \times \operatorname{Ar} \underline{\mathrm{H}}) ; \delta_{\mathrm{C}}(125 \mathrm{MHz}$, $\left.\mathrm{CDCl}_{3}\right) 38.3\left(\underline{\mathrm{C}} \mathrm{H}_{2} \mathrm{~N}\right), 39.3\left(\underline{\mathrm{CH}}_{2} \mathrm{CH}_{2} \mathrm{SO}_{2} \mathrm{Ph}\right), 52.7\left(\underline{\mathrm{CH}}_{2} \mathrm{SO}_{2}\right), 55.4\left(\underline{\mathrm{C}}{ }_{3} \mathrm{O}\right), 55.7(2 \times$ $\left.\underline{\mathrm{CH}}_{3} \mathrm{O}\right), 60.0\left(\underline{\mathrm{CH}}_{2} \mathrm{O}\right), 90.3(2 \times \mathrm{Ar} \underline{\mathrm{CH}}), 102.6(\mathrm{Ar} \underline{\mathrm{C}}), 127.9(2 \times \mathrm{Ar} \underline{\mathrm{CH}}), 129.3(2 \times$ 
$\mathrm{Ar} \underline{\mathrm{CH}}), 133.8(\mathrm{Ar} \underline{\mathrm{CH}}), 139.1(\mathrm{Ar} \underline{\mathrm{C}}), 159.6\left(2 \times \underline{\mathrm{C}}\left(\mathrm{OCH}_{3}\right)\right), 161.9\left(\underline{\mathrm{C}}\left(\mathrm{OCH}_{3}\right)\right), 172.0$ (NCO); $v_{\max }\left(\mathrm{CH}_{2} \mathrm{Cl}_{2}\right.$ evaporated film/ $\left.\mathrm{cm}^{-1}\right) 3432$ (broad, $\left.\mathrm{OH}\right), 2943$ and $2842(\mathrm{CH})$, 1642 (amide CO), $1610(\mathrm{C}=\mathrm{C}) ; \mathrm{m} / \mathrm{z}\left(\mathrm{ES}^{+}\right) 446(100 \%, \mathrm{M}+\mathrm{Na}), 424\left(72 \%, \mathrm{MH}^{+}\right), 181$ (10\%); $\mathrm{C}_{20} \mathrm{H}_{26} \mathrm{NO}_{7} \mathrm{~S}\left(\mathrm{MH}^{+}\right)$found 424.1429, expected 424.1424; mp (EtOH), 134.8$135.9^{\circ} \mathrm{C}$.

\section{2-Hydroxy- $N$-(2,4-dimethoxybenzyl)- $N$-(2-phenylsulfonylethyl)acetamide 41}
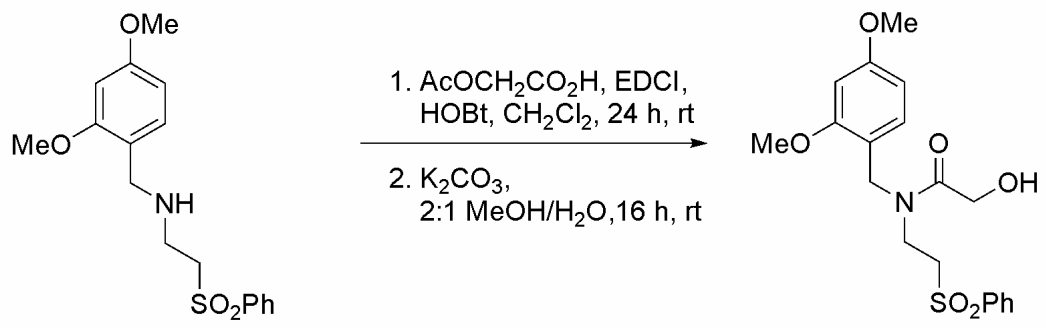

Following general procedure A: N-2,4-Dimethoxybenzyl- $N$-(2-phenylsulfonylethyl) amine $(3.60 \mathrm{~g}, 10.7 \mathrm{mmol}, 1 \mathrm{eq})$ was stirred with acetoxyacetic acid $(1.60 \mathrm{~g}, 12.9 \mathrm{mmol}$, $1.2 \mathrm{eq}), \mathrm{HOBt}(290 \mathrm{mg}, 2.15 \mathrm{mmol}, 0.2 \mathrm{eq})$ and $\mathrm{EDCI}$ ( $2.45 \mathrm{~g}, 12.9 \mathrm{mmol}, 1.2 \mathrm{eq})$. The resulting crude amide $(4.67 \mathrm{~g}, 10.7 \mathrm{mmol}, 1 \mathrm{eq})$ was stirred with $\mathrm{K}_{2} \mathrm{CO}_{3}(6.30 \mathrm{~g}, 45.6$ mmol, 4 eq). Recrystallisation (EtOH) of the cream solid afforded 2-hydroxy- $N$-(2,4dimethoxybenzyl)- $N$-(2-phenylsulfonylethyl)acetamide 41 as a white solid (2.54 g, 6.46 mmol, $60 \%$ over two steps).

$\delta_{\mathrm{H}}\left(300 \mathrm{MHz}, \mathrm{CDCl}_{3}\right.$, rotamer ratio 15:1) $3.15-3.30$ (minor, $2 \mathrm{H}, \mathrm{t}, J=7.1 \mathrm{~Hz}, \mathrm{C}_{2} \mathrm{SO}_{2}$ and major, $2 \mathrm{H}, \mathrm{m}, \mathrm{C}_{2} \mathrm{SO}_{2}$ ), $3.38-3.66$ (minor, $2 \mathrm{H}, \mathrm{m}, \mathrm{C}_{2} \mathrm{CH}_{2} \mathrm{SO}_{2}$ and major, $2 \mathrm{H}, \mathrm{m}$, $\mathrm{C}_{2} \mathrm{CH}_{2} \mathrm{SO}_{2}$ ), 3.71 (minor, $3 \mathrm{H}, \mathrm{s}, \mathrm{C}_{3} \mathrm{O}$ ), 3.74 (major, $3 \mathrm{H}, \mathrm{s}, \mathrm{C}_{3} \mathrm{O}$ ), 3.75 (minor, $3 \mathrm{H}, \mathrm{s}$, $\mathrm{C}_{3} \mathrm{O}$ ), 3.78 (major, $3 \mathrm{H}, \mathrm{s}, \mathrm{C}_{3} \mathrm{O}$ ), 4.02 (minor, $2 \mathrm{H}, \mathrm{s}, \mathrm{C}_{2} \mathrm{OH}$ ), 4.19 (major, $2 \mathrm{H}, \mathrm{s}$, $\mathrm{C}_{2} \mathrm{OH}$ ), 4.20 (major, $2 \mathrm{H}, \mathrm{s}, \mathrm{C}_{2} \mathrm{~N}$ ), 4.44 (minor, $2 \mathrm{H}, \mathrm{s}, \mathrm{C}_{2} \mathrm{~N}$ ), $6.36-6.43$ (minor, $2 \mathrm{H}$, m, $2 \times \operatorname{Ar} \underline{H}$ and major, $2 \mathrm{H}, \mathrm{m}, 2 \times \mathrm{Ar} \underline{\mathrm{H}}$ ), 6.93 (major, $1 \mathrm{H}, \mathrm{d}, \mathrm{J}=7.9 \mathrm{~Hz}, \operatorname{Ar} \underline{\mathrm{H}}$ ), 7.11 (minor, $1 \mathrm{H}, \mathrm{d}, \mathrm{J}=9.3 \mathrm{~Hz}, \mathrm{Ar} \underline{\mathrm{H}}$ ), $7.49-7.59$ (minor, $2 \mathrm{H}, \mathrm{m}, 2 \times \mathrm{Ar} \underline{\mathrm{H}}$ and major, $2 \mathrm{H}, \mathrm{m}, 2$ $\times \operatorname{Ar} \underline{\mathrm{H}}$ ), $7.61-7.71$ (minor, 1H, m, Ar$\underline{\mathrm{H}}$ and major, 1H, m, Ar$\underline{\mathrm{H}}$ ), $7.80-7.86$ (minor, $2 \mathrm{H}, \mathrm{m}, 2 \times \mathrm{Ar} \underline{\mathrm{H}}$ and major, $2 \mathrm{H}, \mathrm{m}, 2 \times \mathrm{Ar} \underline{\mathrm{H}}) ; \delta_{\mathrm{C}}\left(75 \mathrm{MHz}, \mathrm{CDCl}_{3}\right)$ major rotamer 40.5 
$\left(\underline{\mathrm{CH}_{2}} \mathrm{CH}_{2} \mathrm{SO}_{2}\right), 46.4\left(\underline{\mathrm{CH}}_{2} \mathrm{~N}\right), 53.0\left(\underline{\mathrm{CH}_{2}} \mathrm{SO}_{2}\right), 55.6\left(\underline{\left.\mathrm{CH}_{3} \mathrm{O}\right),} 55.6\left(\underline{\mathrm{CH}}_{3} \mathrm{O}\right), 60.3\left(\underline{\mathrm{CH}}_{2} \mathrm{OH}\right)\right.$, $99.0(\mathrm{Ar} \underline{\mathrm{CH}}), 104.5(\mathrm{Ar} \underline{\mathrm{CH}}), 115.2(\mathrm{Ar} \underline{\mathrm{C}}), 128.0(2 \times \mathrm{Ar} \underline{\mathrm{CH}}), 129.6$ (2 $\times$ ArCH$), 130.5$

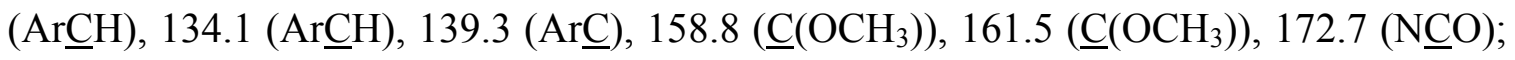
$v_{\max }\left(\mathrm{CH}_{2} \mathrm{Cl}_{2}\right.$ evaporated film/ $\left.\mathrm{cm}^{-1}\right) 3431$ (broad, $\mathrm{OH}$ ), 2936 and $2839(\mathrm{CH}), 1648$ (amide $\mathrm{CO}), 1614$ and $1588(\mathrm{C}=\mathrm{C}) ; \mathrm{m} / \mathrm{z}\left(\mathrm{CI}^{+}\right) 394\left(\mathrm{MH}^{+}, 100 \%\right), 151(57 \%) ; \mathrm{m} / \mathrm{z}\left(\mathrm{EI}^{+}\right) 151$ $(100 \%) ; \mathrm{C}_{19} \mathrm{H}_{24} \mathrm{NO}_{6} \mathrm{~S}\left(\mathrm{MH}^{+}\right)$found 394.1318, expected 394.1319; mp (EtOH) 122.3$123.9^{\circ} \mathrm{C}$.

\section{$N$-Tert-butyl-2-hydroxy- $N$-(2,4-dimethoxybenzyl)acetoxyacetamide 42}
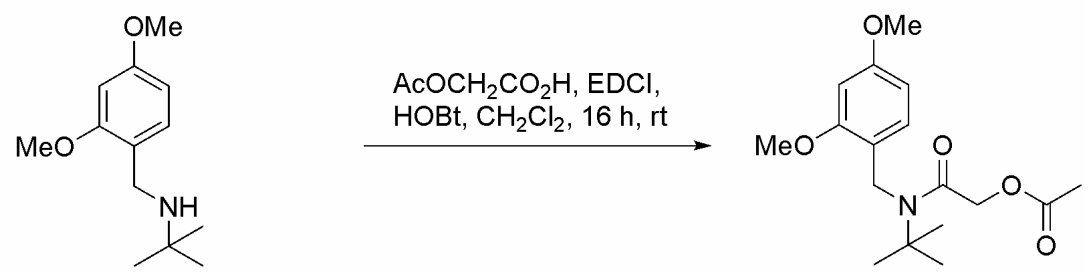

Acetoxyacetyl chloride $(0.72 \mathrm{ml}, 6.73 \mathrm{mmol}, 1.5 \mathrm{eq})$ and triethylamine $(1.13 \mathrm{ml}, 8.07$ mmol, 1.8 eq) were added to $N$-tert-butyl-2,4-dimethoxybenzylamine (1.0 g, $4.48 \mathrm{mmol}$, $1 \mathrm{eq})$ in THF $(25 \mathrm{ml})$ at $0{ }^{\circ} \mathrm{C}$. The resulting solution was stirred at room temperature for 16 hours. Filtration and washing of the reaction mixture with $\mathrm{Et}_{2} \mathrm{O}(3 \times 20 \mathrm{ml})$ and concentration of the filtrate in vacuo gave the crude acetoxyacetamide as a brown oil. Purification by flash column chromatography (3:3:1 petroleum ether/ $\mathrm{CH}_{2} \mathrm{Cl}_{2} / \mathrm{EtOAc}$ ) afforded $N$-tert-butyl-2-hydroxy- $N$-(2,4-dimethoxybenzyl)acetoxyacetamide 42 as a yellow solid (1.20 g, $3.72 \mathrm{mmol}, 83 \%)$.

$\delta_{\mathrm{H}}\left(500 \mathrm{MHz}, \mathrm{CDCl}_{3}\right) 1.43\left(9 \mathrm{H}, \mathrm{s}, 3 \times \mathrm{C}_{3} \mathrm{C}\right), 2.16\left(3 \mathrm{H}, \mathrm{s}, \mathrm{CH}_{3} \mathrm{C}=\mathrm{O}\right), 3.81(3 \mathrm{H}, \mathrm{s}$, $\left.\mathrm{C}_{3} \mathrm{O}\right), 3.82\left(3 \mathrm{H}, \mathrm{s}, \mathrm{C}_{3} \mathrm{O}\right), 4.39\left(2 \mathrm{H}, \mathrm{s}, \mathrm{CH}_{2} \mathrm{~N}\right), 4.62\left(2 \mathrm{H}, \mathrm{s}, \mathrm{C}_{2} \mathrm{O}\right), 6.45(1 \mathrm{H}, \mathrm{d}, J=2.5$ $\left.\mathrm{Hz},\left(\mathrm{CH}_{3} \mathrm{O}\right) \mathrm{CC} \underline{\mathrm{HC}}\left(\mathrm{OCH}_{3}\right)\right), 6.52\left(1 \mathrm{H}, \mathrm{dd}, J=8.5 \mathrm{~Hz}, 2.5 \mathrm{~Hz},\left(\mathrm{CH}_{3} \mathrm{O}\right) \mathrm{CC} \underline{\mathrm{HCH}}\right), 7.20$ $\left(1 \mathrm{H}, \mathrm{d}, J=8.5 \mathrm{~Hz},\left(\mathrm{CH}_{3} \mathrm{O}\right) \mathrm{CCHC} \underline{\mathrm{H}}\right) ; \delta_{\mathrm{C}}\left(125 \mathrm{MHz}, \mathrm{CDCl}_{3}\right) 20.6\left(\mathrm{CH}_{3} \mathrm{CO}_{2}\right), 28.2(3 \times$

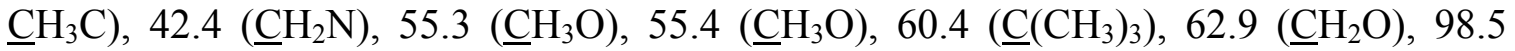
$\left(\left(\mathrm{CH}_{3} \mathrm{O}\right) \mathrm{C} \underline{\mathrm{CHC}}\left(\mathrm{OCH}_{3}\right)\right), 103.8\left(\left(\mathrm{CH}_{3} \mathrm{O}\right) \mathrm{C} \underline{\mathrm{CHCH}}\right), 118.3(\mathrm{Ar} \underline{\mathrm{C}}), 126.9\left(\left(\mathrm{CH}_{3} \mathrm{O}\right) \mathrm{CCHC} H\right)$,

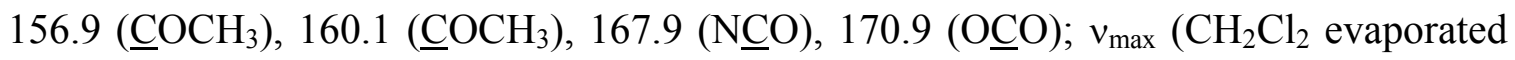
film, $\mathrm{cm}^{-1}$ ) 2963 and $2935(\mathrm{CH}), 1749$ (ester $\mathrm{C}=\mathrm{O}$ ), 1668 (amide $\mathrm{C}=\mathrm{O}$ ), 1616 and 1589 
$(\mathrm{C}=\mathrm{C}) ; \mathrm{m} / \mathrm{z}\left(\mathrm{CI}^{+}\right) 324\left(\mathrm{MH}^{+}, 100 \%\right), 266$ (23\%), $174(21 \%), 151(100 \%) ; \mathrm{m} / \mathrm{z}\left(\mathrm{EI}^{+}\right) 266$ (89\%), 166 (21\%), 151 (27\%), 57 (48\%), 43 (100\%); $\mathrm{C}_{17} \mathrm{H}_{26} \mathrm{NO}_{5}\left(\mathrm{MH}^{+}\right)$found 324.1808, expected 324.1805; $\mathrm{mp}(\mathrm{MeOH}) 87.2-88.4{ }^{\circ} \mathrm{C}$.

\section{$N$-Tert-butyl-2-hydroxy- $N$-(2,4-dimethoxybenzyl)acetamide 43}

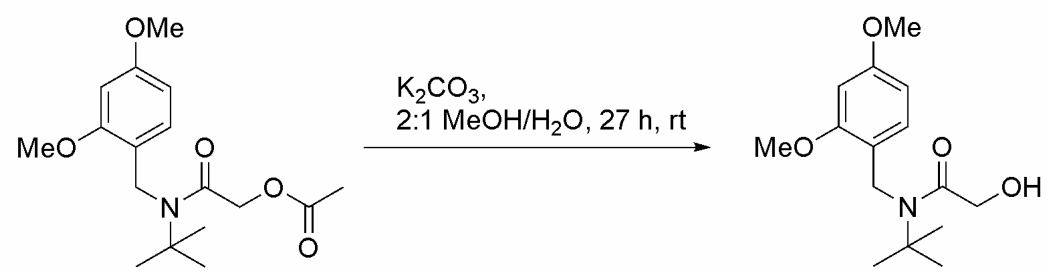

$N$-tert-Butyl-2-hydroxy- $N$-(2,4-dimethoxybenzyl)acetoxyacetamide $42(1.10$ g, 3.41 mmol, 1 eq) was stirred with $\mathrm{K}_{2} \mathrm{CO}_{3}\left(1.83 \mathrm{~g}, 13.2 \mathrm{mmol}\right.$, 4 eq) in 2:1 MeOH$/ \mathrm{H}_{2} \mathrm{O}(15 \mathrm{ml})$ for 27 hours. $\mathrm{H}_{2} \mathrm{O}(40 \mathrm{ml})$ was added and the aqueous layer was extracted with EtOAc (3 $\times 50 \mathrm{ml})$. The combined organic layers were dried $\left(\mathrm{Na}_{2} \mathrm{SO}_{4}\right)$ and concentrated in vacuo to afford $\mathrm{N}$-tert-butyl-2-hydroxy- $\mathrm{N}$-(2,4-dimethoxybenzyl)acetamide $\mathbf{4 3}$ as a cream solid (652 mg, $2.32 \mathrm{mmol}, 68 \%$ ). No further purification was required.

$\delta_{\mathrm{H}}\left(500 \mathrm{MHz}, \mathrm{CDCl}_{3}\right) 1.46\left(9 \mathrm{H}, \mathrm{s}, 3 \times \mathrm{C}_{3} \mathrm{C}\right), 3.81\left(3 \mathrm{H}, \mathrm{s}, \underline{\mathrm{C}}_{3} \mathrm{O}\right), 3.82\left(3 \mathrm{H}, \mathrm{s}, \mathrm{C}_{3} \mathrm{O}\right)$, $4.06\left(2 \mathrm{H}, \mathrm{s}, \mathrm{C}_{2} \mathrm{O}\right), 4.33\left(2 \mathrm{H}, \mathrm{s}, \underline{\mathrm{C}}_{2} \mathrm{~N}\right), 6.44-6.49(2 \mathrm{H}, \mathrm{m}, 2 \times \mathrm{Ar} \underline{\mathrm{H}}), 7.01(1 \mathrm{H}, \mathrm{d}, J=8.2$ $\mathrm{Hz}, \mathrm{Ar} \underline{\mathrm{H}}) ; \delta_{\mathrm{C}}\left(125 \mathrm{MHz}, \mathrm{CDCl}_{3}\right) 28.2\left(3 \times \underline{\mathrm{CH}}_{3} \mathrm{C}\right), 41.5\left(\underline{\mathrm{CH}}_{2} \mathrm{~N}\right), 55.3\left(\underline{\mathrm{CH}}_{3} \mathrm{O}\right), 55.4$ $\left(\underline{\mathrm{C}} \mathrm{H}_{3} \mathrm{O}\right), 58.5\left(\underline{\mathrm{CH}}_{2} \mathrm{O}\right), 61.0\left(\left(\mathrm{CH}_{3}\right)_{3} \underline{\mathrm{C}}\right), 98.5(\mathrm{Ar} \underline{\mathrm{CH}}), 103.9$ (Ar $\left.\underline{\mathrm{CH}}\right), 118.3(\mathrm{Ar} \underline{\mathrm{C}}), 126.4$ $(\mathrm{Ar} \underline{\mathrm{CH}}), 156.9\left(\underline{\mathrm{C}}\left(\mathrm{OCH}_{3}\right)\right), 160.1\left(\underline{\mathrm{C}}\left(\mathrm{OCH}_{3}\right)\right), 172.8(\mathrm{~N} \underline{\mathrm{CO}}) ; v_{\max }\left(\mathrm{CH}_{2} \mathrm{Cl}_{2}\right.$ evaporated film $/ \mathrm{cm}^{-1}$ ) 3411 (broad, OH), 2966 and $2839(\mathrm{CH}), 1649$ (amide $\mathrm{C}=\mathrm{O}$ ), 1618 and 1590 $(\mathrm{C}=\mathrm{C}) ; \mathrm{m} / \mathrm{z}\left(\mathrm{ES}^{+}\right) 304(41 \%, \mathrm{M}+\mathrm{Na}), 224(6 \%), 151(7 \%) ; \mathrm{C}_{15} \mathrm{H}_{23} \mathrm{NO}_{4} \mathrm{Na}(\mathrm{M}+\mathrm{Na})$ found 304.1531, expected 304.1519, $\mathrm{mp}(\mathrm{MeOH}) 125.5-127.3^{\circ} \mathrm{C}$. 


\section{2-Hydroxy- $N$-iso-propyl- $N$-(2,4-dimethoxybenzyl)acetamide 44}
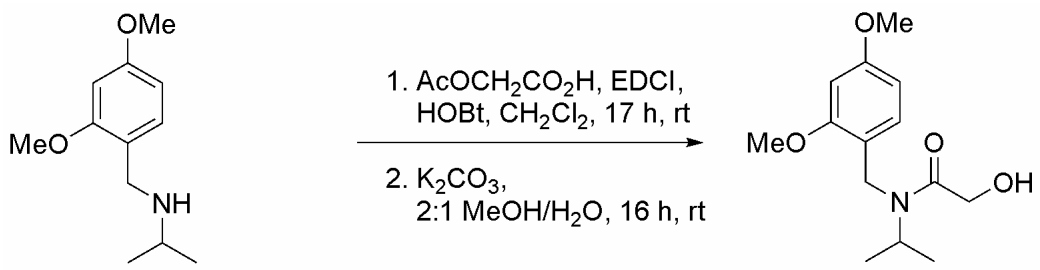

Following general procedure A: $N$-iso-Propyl- $N$-2,4-dimethoxybenzylamine (1.64 g, 7.85 mmol, 1 eq) was stirred with acetoxyacetic acid (1.11 g, 9.42 mmol, $1.2 \mathrm{eq}), \mathrm{HOBt}$ (212 $\mathrm{mg}, 1.57 \mathrm{mmol}, 0.2 \mathrm{eq})$ and EDCI (1.81 g, $9.42 \mathrm{mmol}, 1.2 \mathrm{eq})$. The resulting crude amide (2.42 g, 7.85 mmol, 1 eq) was stirred with $\mathrm{K}_{2} \mathrm{CO}_{3}$ (4.40 g, $\left.31.8 \mathrm{mmol}, 4 \mathrm{eq}\right) . \quad 2$ hydroxy- $N$-iso-propyl- $N$-(2,4-dimethoxybenzyl)acetamide $\mathbf{4 4}$ was obtained as a cloudy oil (1.65 g, $6.18 \mathrm{mmol}, 79 \%$ over two steps). No further purification was required.

$\delta_{\mathrm{H}}\left(300 \mathrm{MHz}, \mathrm{CDCl}_{3}\right.$, rotamer ratio 2:1) 1.17 (major, $6 \mathrm{H}, \mathrm{d}, J=6.9 \mathrm{~Hz}, 2 \times \mathrm{CH}_{3} \mathrm{CH}$ ), 1.18 (minor, $6 \mathrm{H}, \mathrm{d}, J=6.6 \mathrm{~Hz}, 2 \times \mathrm{C}_{3} \mathrm{CH}$ ), 3.79-3.90 (minor, $1 \mathrm{H}, \mathrm{m}, \mathrm{C} \underline{\mathrm{H}}\left(\mathrm{CH}_{3}\right)_{2}$ ), 3.82 (minor, $3 \mathrm{H}, \mathrm{s}, \mathrm{C}_{3} \mathrm{O}$ ), 3.84 (major, $3 \mathrm{H}, \mathrm{s}, \mathrm{C}_{3} \mathrm{O}$ ), 3.86 (major, $3 \mathrm{H}, \mathrm{s}, \mathrm{C}_{3} \mathrm{O}$ ), 3.87 (minor, $3 \mathrm{H}, \mathrm{s}, \mathrm{C}_{3} \mathrm{O}$ ), 4.12 (major, $2 \mathrm{H}, \mathrm{s}, \mathrm{C}_{2} \mathrm{O}$ ), 4.26 (major, $2 \mathrm{H}, \mathrm{s}, \mathrm{C}_{2} \mathrm{~N}$ ), 4.34 (minor, $2 \mathrm{H}, \mathrm{s}, \mathrm{C}_{2} \mathrm{O}$ ), 4.58 (minor, $2 \mathrm{H}, \mathrm{s}, \underline{\mathrm{C}}_{2} \mathrm{~N}$ ), 4.72 (major, $1 \mathrm{H}$, septet, $J=6.9 \mathrm{~Hz}$, $\left.\mathrm{C} \underline{\mathrm{H}}\left(\mathrm{CH}_{3}\right)_{2}\right), 6.43-6.52$ (major, $2 \mathrm{H}, \mathrm{m}, 2 \times \mathrm{Ar} \underline{\mathrm{H}}$ and minor, $2 \mathrm{H}, \mathrm{m}, 2 \times \mathrm{Ar} \underline{\mathrm{H}}$ ), 6.98-7.08 (major, $1 \mathrm{H}, \mathrm{m}, \mathrm{Ar} \underline{\mathrm{H}}$ and minor, $1 \mathrm{H}, \mathrm{m}, \mathrm{Ar} \underline{\mathrm{H}}) ; \delta_{\mathrm{C}}\left(75 \mathrm{MHz}, \mathrm{CDCl}_{3}\right)$ major rotamer $20.2(2$ $\left.\times \underline{\mathrm{C}}_{3} \mathrm{CH}\right), 40.7\left(\underline{\mathrm{CH}}_{2} \mathrm{~N}\right), 47.7\left(\underline{\mathrm{CH}}\left(\mathrm{CH}_{3}\right)_{2}\right), 55.5\left(\underline{\mathrm{CH}}_{3} \mathrm{O}\right), 55.6\left(\underline{\mathrm{CH}}_{3} \mathrm{O}\right), 60.6\left(\underline{\mathrm{CH}}_{2} \mathrm{O}\right)$, $98.8(\mathrm{Ar} \underline{\mathrm{CH}}), 104.1(\mathrm{Ar} \underline{\mathrm{CH}}), 117.5(\mathrm{Ar} \underline{\mathrm{C}}), 127.5(\mathrm{Ar} \underline{\mathrm{CH}}), 157.8\left(\underline{\mathrm{C}}\left(\mathrm{OCH}_{3}\right)\right), 160.6$ $\left(\underline{\mathrm{C}}\left(\mathrm{OCH}_{3}\right)\right), 172.7(\mathrm{NCO}) ; v_{\max }\left(\right.$ neat $\left./ \mathrm{cm}^{-1}\right) 3413$ (broad, OH), 2970 and $2936(\mathrm{CH}), 1644$ (amide $\mathrm{C}=\mathrm{O}), 1620$ and 1590 ( $\mathrm{Ar} \mathrm{C}=\mathrm{C}) ; \mathrm{m} / \mathrm{z}\left(\mathrm{ES}^{+}\right) 290(16 \%, \mathrm{M}+\mathrm{Na}), 210$ (53\%), 151 (19\%); $\mathrm{C}_{14} \mathrm{H}_{21} \mathrm{NO}_{4} \mathrm{Na}(\mathrm{M}+\mathrm{Na})$ found 290.1375, expected 290.1363. 


\section{2-Hydroxy- $N$-iso-propyl-(2,4,5-trimethoxybenzyl)acetamide 45}
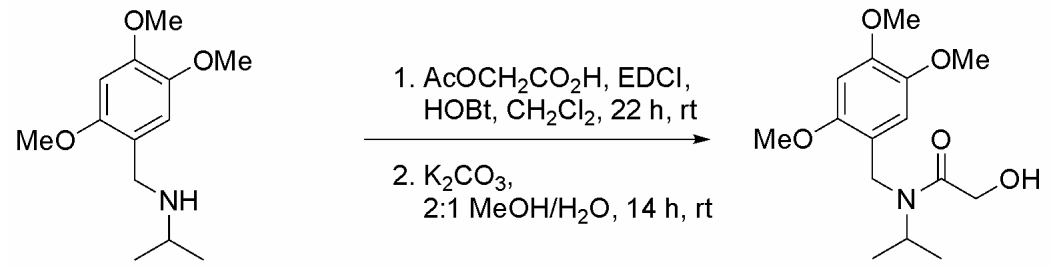

Following general procedure A: N-iso-Propyl-(2,4,5-trimethoxybenzyl) amine (3.05 g, $12.8 \mathrm{mmol}, 1 \mathrm{eq})$ was stirred with acetoxyacetic acid (1.81 g, $15.3 \mathrm{mmol}, 1.2 \mathrm{eq}), \mathrm{HOBt}$ (345 mg, $2.55 \mathrm{mmol}, 0.2 \mathrm{eq})$ and EDCI (2.94 g, $15.3 \mathrm{mmol}, 1.2 \mathrm{eq})$. The resulting crude amide (3.49 g, $10.3 \mathrm{mmol}, 1 \mathrm{eq})$ was stirred with $\mathrm{K}_{2} \mathrm{CO}_{3}(5.69 \mathrm{~g}, 41.2 \mathrm{mmol}, 4 \mathrm{eq})$ and recrystallisation of the crude product $(\mathrm{EtOH})$ afforded 2-hydroxy- $\mathrm{N}$-iso-propyl-(2,4,5trimethoxybenzyl)acetamide 45 as a cream solid (1.97 g, $6.63 \mathrm{mmol}, 52 \%$ over two steps).

$\delta_{\mathrm{H}}\left(500 \mathrm{MHz}, \mathrm{CDCl}_{3}\right.$, rotamer ratio 3:2) 1.15 (minor, $6 \mathrm{H}, \mathrm{d}, J=6.9 \mathrm{~Hz}, 2 \times \mathrm{C}_{3} \mathrm{CH}$ ), 1.16 (major, $6 \mathrm{H}, \mathrm{d}, J=6.9 \mathrm{~Hz}, 2 \times \mathrm{C}_{3} \mathrm{CH}$ ), 3.67 (major , $1 \mathrm{H}, \mathrm{t}, J=4.4 \mathrm{~Hz}, \mathrm{CH}_{2} \mathrm{O} \underline{\mathrm{H}}$ ), 3.73-3.79 (minor, $1 \mathrm{H}, \mathrm{m}, \mathrm{C} \underline{\mathrm{H}}\left(\mathrm{CH}_{3}\right)_{2}$ ), 3.79 (minor, $3 \mathrm{H}, \mathrm{s}, \mathrm{CH}_{3} \mathrm{O}$ ), 3.80 (major, $3 \mathrm{H}$, s, $\mathrm{CH}_{3} \mathrm{O}$ ), 3.84 (major, $3 \mathrm{H}, \mathrm{s}, \mathrm{CH}_{3} \mathrm{O}$ ), 3.85 (minor, $3 \mathrm{H}, \mathrm{s}, \mathrm{C}_{3} \mathrm{O}$ ), 3.88 (minor, $3 \mathrm{H}, \mathrm{s}$, $\mathrm{C}_{3} \mathrm{O}$ ), 3.89 (minor, $1 \mathrm{H}, \mathrm{t}, J=4.1 \mathrm{~Hz}, \mathrm{CH}_{2} \mathrm{O} \underline{\mathrm{H}}$ ), 3.90 (major, $3 \mathrm{H}, \mathrm{s}, \mathrm{C}_{3} \mathrm{O}$ ), 4.09 (major, $2 \mathrm{H}, \mathrm{d}, J=4.4 \mathrm{~Hz}, \underline{\mathrm{C}}_{2} \mathrm{OH}$ ), 4.25 (major, $2 \mathrm{H}, \mathrm{s}, \mathrm{C}_{2} \mathrm{~N}$ ), 4.30 (minor, $2 \mathrm{H}, \mathrm{d}, J=4.1 \mathrm{~Hz}$,

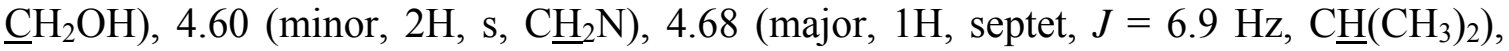

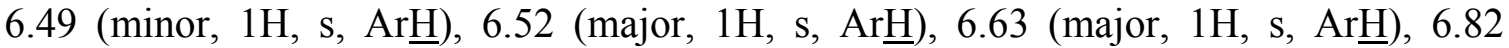
(minor, $1 \mathrm{H}, \mathrm{s}, \mathrm{Ar} \underline{\mathrm{H}}) ; \delta_{\mathrm{C}}\left(125 \mathrm{MHz}, \mathrm{CDCl}_{3}\right)$ major rotamer $20.0\left(2 \times \underline{\mathrm{CH}_{3} \mathrm{CH}}\right), 37.8$

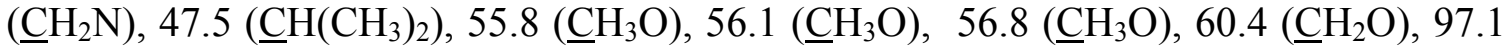

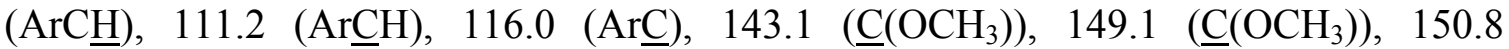
$\left(\underline{\mathrm{C}}\left(\mathrm{OCH}_{3}\right)\right), 172.4(\mathrm{NCO}) ; v_{\max }\left(\mathrm{CH}_{2} \mathrm{Cl}_{2}\right.$ evaporated film, $\left.\mathrm{cm}^{-1}\right) 3414$ (broad, $\left.\mathrm{OH}\right), 2937$ and $2964(\mathrm{CH}), 1643$ (amide $\mathrm{C}=\mathrm{O}), 1516(\mathrm{C}=\mathrm{C}) ; \mathrm{m} / \mathrm{z}\left(\mathrm{CI}^{+}\right) 298\left(\mathrm{MH}^{+}, 100 \%\right), 254$ (13\%), 181 (56\%, 117 (12\%); m/z (EI ) 195 (37\%), 181 (58\%), 77 (52\%), 50 (100\%); $\mathrm{C}_{15} \mathrm{H}_{24} \mathrm{NO}_{5}\left(\mathrm{MH}^{+}\right)$found 298.1654, expected 298.1649; anal. calcd. for $\mathrm{C}_{15} \mathrm{H}_{23} \mathrm{NO}_{5}$ : C $(60.53 \%), \mathrm{H}(8.06 \%) \mathrm{N}(4.68 \%)$; found C (60.59\%), H (7.80\%), N (4.71\%); mp (EtOH) $74.5-75-6^{\circ} \mathrm{C}$. 


\section{2-Hydroxy- $N$-iso-propyl-(2,3,4-trimethoxybenzyl)acetamide 46}
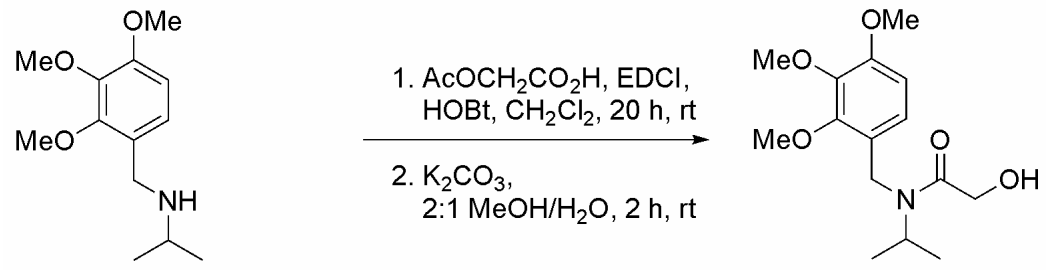

Following general procedure A: $N$-iso-Propyl-(2,3,4-trimethoxybenzyl) amine (3.00 g, $12.6 \mathrm{mmol}, 1 \mathrm{eq})$ was stirred with acetoxyacetic acid (1.78 g, $15.1 \mathrm{mmol}, 1.2 \mathrm{eq}), \mathrm{HOBt}$ (340 mg, $2.51 \mathrm{mmol}, 0.2 \mathrm{eq})$ and EDCI (2.89 g, $15.1 \mathrm{mmol}, 1.2 \mathrm{eq})$. The resulting crude amide (4.17 g, $12.3 \mathrm{mmol}, 1 \mathrm{eq})$ was stirred with $\mathrm{K}_{2} \mathrm{CO}_{3}(6.80 \mathrm{~g}, 49.2 \mathrm{mmol}, 4 \mathrm{eq})$ to afford 2-hydroxy- $\mathrm{N}$-iso-propyl-(2,3,4-trimethoxybenzyl)acetamide 46 as a colourless oil (3.33 g, $11.2 \mathrm{mmol}, 89 \%$ over two steps).

$\delta_{\mathrm{H}}\left(500 \mathrm{MHz}, \mathrm{CDCl}_{3}\right.$, rotamer ratio 1:1) 1.14 (both rotamers, $12 \mathrm{H}, \mathrm{d}, J=6.6 \mathrm{~Hz}, 4 \times$ $\mathrm{C}_{3} \mathrm{CH}$ ), 3.78 (one rotamer, $1 \mathrm{H}$, septet, $6.6 \mathrm{~Hz}, \underline{\mathrm{CH}}\left(\mathrm{CH}_{3}\right)_{2}$ ), 3.82 (one rotamer, $3 \mathrm{H}$, s, $\mathrm{C}_{3} \mathrm{O}$ ), 3.84 (one rotamer, $3 \mathrm{H}, \mathrm{s}, \mathrm{C}_{3} \mathrm{O}$ ), 3.85 (one rotamer, $3 \mathrm{H}, \mathrm{s}, \mathrm{CH}_{3} \mathrm{O}$ ), 3.86 (one rotamer, $3 \mathrm{H}, \mathrm{s}, \mathrm{CH}_{3} \mathrm{O}$ ), 3.92 (one rotamer, $3 \mathrm{H}, \mathrm{s}, \mathrm{CH}_{3} \mathrm{O}$ ), 3.93 (one rotamer, $3 \mathrm{H}, \mathrm{s}$, $\mathrm{C}_{3} \mathrm{O}$ ), 4.09 (one rotamer, $2 \mathrm{H}, \mathrm{s}, \mathrm{C}_{2} \mathrm{O}$ ), 4.25 (one rotamer, $2 \mathrm{H}, \mathrm{s}, \mathrm{C}_{2} \mathrm{~N}$ ), $4.30(2 \mathrm{H}, \mathrm{s}$, $\mathrm{C}_{2} \mathrm{O}$ ), 4.57 (one rotamer, $2 \mathrm{H}, \mathrm{s}, \mathrm{C}_{2} \mathrm{~N}$ ), 4.67 (one rotamer, $1 \mathrm{H}$, septet, $J=6.6 \mathrm{~Hz}$, $\mathrm{C} \underline{\mathrm{H}}\left(\mathrm{CH}_{3}\right)_{2}$ ), 6.59 (one rotamer, $1 \mathrm{H}, \mathrm{d}, J=8.7 \mathrm{~Hz}, \mathrm{Ar} \underline{\mathrm{H}}$ ), 6.61 (one rotamer, $1 \mathrm{H}, \mathrm{d}, J=8.7$ $\mathrm{Hz}, \mathrm{Ar} \underline{\mathrm{H}}$ ), 6.75 (one rotamer, $1 \mathrm{H}, \mathrm{d}, J=8.7 \mathrm{~Hz}, \mathrm{Ar} \underline{\mathrm{H}}$ ), 6.78 (one rotamer, $1 \mathrm{H}, \mathrm{d}, J=8.7$ $\mathrm{Hz}, \mathrm{Ar} \underline{\mathrm{H}}) ; \delta_{\mathrm{C}}\left(125 \mathrm{MHz}, \mathrm{CDCl}_{3}\right)$ one rotamer $20.0\left(2 \times \underline{\mathrm{CH}}_{3} \mathrm{CH}\right), 40.4\left(\underline{\mathrm{CH}}_{2} \mathrm{~N}\right), 47.5$

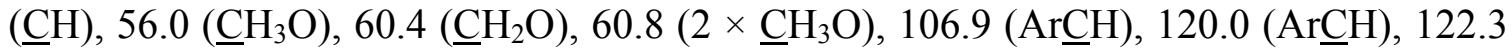

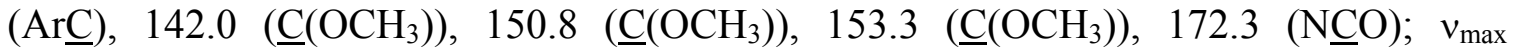
$\left(\right.$ neat $/ \mathrm{cm}^{-1}$ ) 3412 (broad, OH), 2969, 2939 and $2837(\mathrm{CH}), 1644$ (amide $\left.\mathrm{C}=\mathrm{O}\right), 1603$ and $1544(\mathrm{C}=\mathrm{C}) ; \mathrm{m} / \mathrm{z}\left(\mathrm{ES}^{+}\right) 320(79 \%, \mathrm{M}+\mathrm{Na}), 217(12 \%), 151 \quad(13 \%) ; \mathrm{C}_{15} \mathrm{H}_{23} \mathrm{NO}_{5} \mathrm{Na}$ $(\mathrm{M}+\mathrm{Na})$ found 320.1467, expected 320.1468. 


\section{2-Hydroxy- $N$-iso-propyl-(2,4-dimethoxy-3-methylbenzyl)acetamide 47}
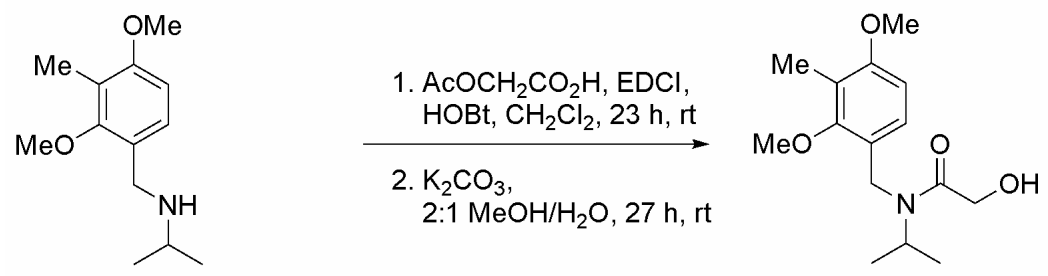

Following general procedure A: $N$-iso-Propyl-(2,4-dimethoxy-3-methylbenzyl) amine (2.40 g, $10.8 \mathrm{mmol}, 1 \mathrm{eq})$ was stirred with acetoxyacetic acid (1.53 g, $12.9 \mathrm{mmol}, 1.2 \mathrm{eq})$, HOBt (291 mg, $2.15 \mathrm{mmol}, 0.2 \mathrm{eq})$ and EDCI (2.48 g, $12.9 \mathrm{mmol}, 1.2 \mathrm{eq})$. The resulting crude amide (3.48 g, $10.8 \mathrm{mmol}, 1 \mathrm{eq})$ was stirred with $\mathrm{K}_{2} \mathrm{CO}_{3}(5.95 \mathrm{~g}, 43.0 \mathrm{mmol}, 4 \mathrm{eq})$ to afford 2-hydroxy- $N$-iso-propyl-(2,4-dimethoxy-3-methylbenzyl)acetamide 47 as a cream amorphous solid (2.57 g, $9.15 \mathrm{mmol}, 85 \%$ over two steps). No further purification was required.

$\delta_{\mathrm{H}}\left(500 \mathrm{MHz}, \mathrm{CDCl}_{3}\right.$, rotamer ratio 3:2) 1.13 (minor, $6 \mathrm{H}, \mathrm{d}, J=6.6 \mathrm{~Hz}, 2 \times \mathrm{C}_{3} \mathrm{CH}$ ), 1.16 (major, $6 \mathrm{H}, \mathrm{d}, J=6.9 \mathrm{~Hz}, 2 \times \mathrm{C}_{3} \mathrm{CH}$ ), 2.16 (minor, $3 \mathrm{H}, \mathrm{s}, \mathrm{ArCH}_{3}$ ), 2.17 (major, $3 \mathrm{H}, \mathrm{s}, \mathrm{ArC}_{3}$ ), 3.74 (major, $3 \mathrm{H}, \mathrm{s}, \mathrm{C}_{3} \mathrm{O}$ ), 3.75 (minor, $3 \mathrm{H}, \mathrm{s}, \mathrm{C}_{3} \mathrm{O}$ ), 3.78-3.84 (minor, $1 \mathrm{H}, \mathrm{m}, \mathrm{C} \underline{\mathrm{H}}\left(\mathrm{CH}_{3}\right)_{2}$ ), 3.80 (minor, $3 \mathrm{H}, \mathrm{s}, \mathrm{C}_{3} \mathrm{O}$ ), 3.82 (major, $3 \mathrm{H}, \mathrm{s}, \mathrm{C}_{3} \mathrm{O}$ ), 4.09 (major, $2 \mathrm{H}, \mathrm{s}, \mathrm{C}_{2} \mathrm{OH}$ ), 4.31 (minor, $2 \mathrm{H}, \mathrm{s}, \underline{\mathrm{CH}_{2}} \mathrm{OH}$ and major, $2 \mathrm{H}, \mathrm{s}, \underline{\mathrm{C}}_{2} \mathrm{~N}$ ), 4.63 (minor, $2 \mathrm{H}$, $\mathrm{s}, \underline{\mathrm{C}}_{2} \mathrm{~N}$ ), 4.71 (major, $1 \mathrm{H}$, septet, $J=6.9 \mathrm{~Hz}, \mathrm{C} \underline{\mathrm{H}}\left(\mathrm{CH}_{3}\right)_{2}$ ), 6.58 (minor, $1 \mathrm{H}, \mathrm{d}, J=8.5 \mathrm{~Hz}$, Ar్), 6.61 (major, 1H, d, $J=8.5 \mathrm{~Hz}, \operatorname{Ar} \underline{\mathrm{H}}$ ), 6.90 (major, 1H, d, $J=8.5 \mathrm{~Hz}, \operatorname{Ar} \underline{\mathrm{H}}$ ), 6.93 (minor, $1 \mathrm{H}, \mathrm{d}, J=8.5 \mathrm{~Hz}, \mathrm{Ar} \underline{\mathrm{H}}) ; \delta_{\mathrm{C}}\left(125 \mathrm{MHz}, \mathrm{CDCl}_{3}\right)$ major rotamer: $8.9\left(\mathrm{Ar}_{\underline{C H}}\right), 19.9$

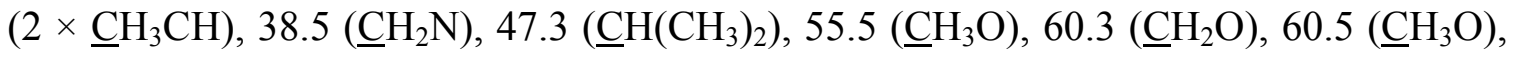

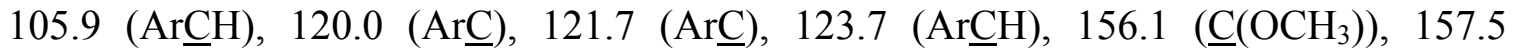
$\left(\underline{\mathrm{C}}\left(\mathrm{OCH}_{3}\right)\right), 172.3(\mathrm{NCO}) ; v_{\max }\left(\mathrm{CH}_{2} \mathrm{Cl}_{2}\right.$ evaporated film/ $\left./ \mathrm{cm}^{-1}\right) 3412$ (broad, OH), 2940 and $2837(\mathrm{CH}), 1644$ (amide $\mathrm{C}=\mathrm{O}), 1604(\mathrm{C}=\mathrm{C}) ; \mathrm{m} / \mathrm{z}\left(\mathrm{CI}^{+}\right) 282\left(\mathrm{MH}^{+}, 100 \%\right), 238$ (19\%), 134 (31\%); m/z (EI ) $238\left(\mathrm{MH}^{+}, 52 \%\right), 180$ (100\%), 165 (73\%), 105 (34\%), 91 (61\%), $77(48 \%) ; \mathrm{C}_{15} \mathrm{H}_{24} \mathrm{NO}_{4}\left(\mathrm{MH}^{+}\right)$found 282.1703, expected 282.1700. 


\section{$N$-(2-Fluoro-4-methoxybenzyl)-2-hydroxy- $N$-iso-propylacetamide 48}
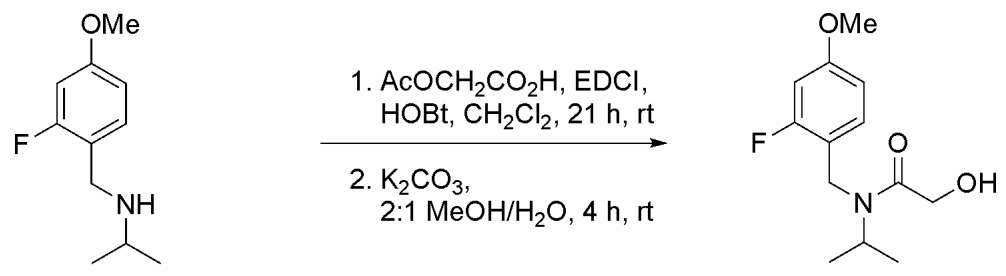

Following general procedure A: $N$-(2-Fluoro-4-methoxybenzyl)- $N$-iso-propylamine $(2.00$ g, $10.2 \mathrm{mmol}$, 1eq) was stirred with acetoxyacetic acid (1.44 g, $12.2 \mathrm{mmol}, 1.2 \mathrm{eq}), \mathrm{HOBt}$ (274 mg, $2.03 \mathrm{mmol}, 0.2 \mathrm{eq})$ and EDCI (2.34 g, $12.2 \mathrm{mmol}, 1.2 \mathrm{eq})$. The resulting crude amide (3.27 g, $11.0 \mathrm{mmol}, 1 \mathrm{eq})$ was stirred with $\mathrm{K}_{2} \mathrm{CO}_{3}(6.09 \mathrm{~g}, 44.0 \mathrm{mmol}, 4 \mathrm{eq})$. Purification by flash column chromatography on silica gel (3:3:1 petroleum ether/ $\mathrm{CH}_{2} \mathrm{Cl}_{2} / \mathrm{EtOAc}$ afforded $\quad \mathrm{N}$-(2-fluoro-4-methoxybenzyl)-2-hydroxy- $\mathrm{N}$-isopropylacetamide 48 as an orange oil (1.99 g, $7.8 \mathrm{mmol}, 76 \%$ over two steps).

$\delta_{\mathrm{H}}\left(500 \mathrm{MHz}, \mathrm{CDCl}_{3}\right.$, rotamer ratio 4:3) 1.08 (minor, $6 \mathrm{H}, \mathrm{d}, J=6.9 \mathrm{~Hz}, \mathrm{CH}_{3} \mathrm{CH}$ ), 1.09 (major, $6 \mathrm{H}, \mathrm{d}, J=6.6 \mathrm{~Hz}, \mathrm{CH}_{3} \mathrm{CH}$ ), $2.65-3.05$ (both rotamers, $1 \mathrm{H}$ and $1 \mathrm{H}$, br s, $\mathrm{O} \underline{\mathrm{H}}$ ), 3.67 - 3.75 (minor, $1 \mathrm{H}, \mathrm{m}, \mathrm{C} \underline{\mathrm{H}}\left(\mathrm{CH}_{3}\right)_{2}$ ), 3.71 (major, $3 \mathrm{H}, \mathrm{s}, \mathrm{C}_{3} \mathrm{O}$ ), 3.73 (minor, $3 \mathrm{H}$, s, $\mathrm{C}_{3} \mathrm{O}$ ), 4.04 (minor, $2 \mathrm{H}, \mathrm{s}, \mathrm{C}_{2} \mathrm{O}$ ), 4.22 (major, $2 \mathrm{H}, \mathrm{s}, \mathrm{C}_{2} \mathrm{O}$ and minor, $2 \mathrm{H}, \mathrm{s}, \mathrm{C}_{2} \mathrm{~N}$ ), 4.52 (major, $2 \mathrm{H}, \mathrm{s}, \mathrm{C}_{2} \mathrm{~N}$ ), 4.61 (major, $1 \mathrm{H}$, septet, $\left.J=6.6 \mathrm{~Hz}, \underline{\mathrm{C}}\left(\mathrm{CH}_{3}\right)_{2}\right), 6.47-6.64$ (major, $2 \mathrm{H}, \mathrm{m}, 2 \times \mathrm{Ar} \underline{\mathrm{H}}$ and minor, $2 \mathrm{H}, \mathrm{m}, 2 \times \mathrm{Ar} \underline{\mathrm{H}}$ ), 6.98 (minor, $1 \mathrm{H}$, apparent $\mathrm{t}, J=8.8$ $\mathrm{Hz}, \operatorname{Ar} \underline{\mathrm{H}}$ ), 7.12 (major, 1H, apparent t, $J=8.8 \mathrm{~Hz}, \mathrm{Ar} \underline{\mathrm{H}}) ; \delta_{\mathrm{C}}\left(125 \mathrm{MHz}, \mathrm{CDCl}_{3}\right.$ ) major

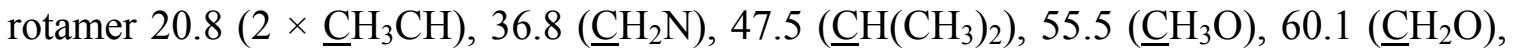
$101.3\left(\mathrm{~d}, J^{2}{ }_{\mathrm{CF}}=25 \mathrm{~Hz}, \mathrm{Ar} \underline{\mathrm{CHCF}}\right), 110.1\left(\mathrm{~d}, J_{\mathrm{CF}}^{4}=3 \mathrm{~Hz},\left(\mathrm{CH}_{3} \mathrm{O}\right) \mathrm{C} \underline{\mathrm{CHCH}}\right), 117.4\left(\mathrm{~d}, J^{2}{ }_{\mathrm{CF}}\right.$ $=14 \mathrm{~Hz}, \mathrm{Ar} \underline{\mathrm{C}}), 129.9\left(\mathrm{~d}, J_{\mathrm{CF}}^{3}=6 \mathrm{~Hz},\left(\mathrm{CH}_{3} \mathrm{O}\right) \mathrm{CCH} \underline{\mathrm{H}}\right), 159.9\left(\mathrm{~d}, J_{\mathrm{CF}}^{3}=11 \mathrm{~Hz}\right.$, $\left.\underline{\mathrm{C}}\left(\mathrm{OCH}_{3}\right)\right), 160.3\left(\mathrm{~d}, J_{\mathrm{CF}}^{1}=245 \mathrm{~Hz}, \mathrm{ArCHCF}\right), 172.0(\mathrm{NCO}) ; v_{\max }\left(\right.$ neat $\left./ \mathrm{cm}^{-1}\right) 3412(\mathrm{OH})$, 2975 and $2938(\mathrm{CH}), 1642$ (amide $\mathrm{C}=\mathrm{O}), 1588(\mathrm{C}=\mathrm{C}) ; \mathrm{m} / \mathrm{z}\left(\mathrm{ES}^{+}\right) 278(100 \%, \mathrm{M}+\mathrm{Na})$, $256\left(4 \%, \mathrm{MH}^{+}\right), 211(26 \%), 139(11 \%) ; \mathrm{C}_{13} \mathrm{H}_{18} \mathrm{NO}_{3} \mathrm{FNa}(\mathrm{M}+\mathrm{Na})$ found 278.1174, expected 278.1163. 


\section{2-Hydroxy- $N$-iso-propyl- $N$-(2,6-dimethoxybenzyl)acetamide 49}
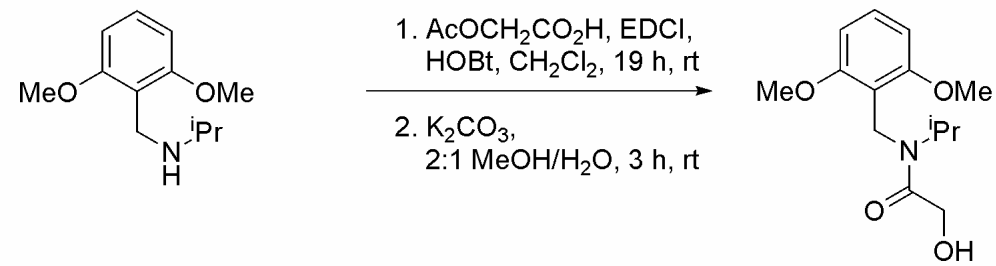

Following general procedure A: $N$-iso-Propyl- $N$-(2,6-dimethoxybenzyl) amine (1.36 g, $6.51 \mathrm{mmol}, 1 \mathrm{eq})$ was stirred with acetoxyacetic acid (0.92 g, $7.81 \mathrm{mmol}, 1.2 \mathrm{eq}), \mathrm{HOBt}$ (176 mg, $1.30 \mathrm{mmol}, 0.2 \mathrm{eq})$ and EDCI (1.50 g, $7.81 \mathrm{mmol}, 1.2 \mathrm{eq})$. The resulting crude amide (1.81 g, $5.86 \mathrm{mmol}, 1 \mathrm{eq})$ was stirred with $\mathrm{K}_{2} \mathrm{CO}_{3}(3.24 \mathrm{~g}, 23.4 \mathrm{mmol}, 4 \mathrm{eq})$ to afford 2-hydroxy- $N$-iso-propyl- $N$-(2,6-dimethoxybenzyl)acetamide 49 as a cream solid ( $2.57 \mathrm{~g}, 3.75 \mathrm{mmol}, 85 \%$ over two steps). No further purification was required.

$\delta_{\mathrm{H}}\left(500 \mathrm{MHz}, \mathrm{CDCl}_{3}\right.$, rotamer ratio 8:1) 1.03 (minor, $6 \mathrm{H}, \mathrm{d}, J=6.6 \mathrm{~Hz}, 2 \times \mathrm{C}_{3} \mathrm{CH}$ ), 1.09 (major, $6 \mathrm{H}, \mathrm{d}, J=6.9 \mathrm{~Hz}, 2 \times \mathrm{CH}_{3} \mathrm{CH}$ ), 3.61 (minor, $1 \mathrm{H}$, septet, $J=6.9 \mathrm{~Hz}$, $\mathrm{C} \underline{\mathrm{H}}\left(\mathrm{CH}_{3}\right)_{2}$ ), 3.80 (minor, $6 \mathrm{H}, \mathrm{s}, 2 \times \underline{\mathrm{CH}}_{3} \mathrm{O}$ ), 3.81 (major, $6 \mathrm{H}, \mathrm{s}, 2 \times \underline{\mathrm{CH}}_{3} \mathrm{O}$ ), 3.90 (major, $1 \mathrm{H}$, septet, $\left.J=6.9 \mathrm{~Hz}, \mathrm{C} \underline{\mathrm{H}}\left(\mathrm{CH}_{3}\right)_{2}\right), 3.92$ (major, $1 \mathrm{H}, \mathrm{t}, J=4.1 \mathrm{~Hz}, \mathrm{CH}_{2} \mathrm{O} \underline{\mathrm{H}}$ ), 4.18 (minor, $\mathrm{d}, J=5.8 \mathrm{~Hz}, \underline{\mathrm{CH}}_{2} \mathrm{OH}$ ), 4.32 (major, $2 \mathrm{H}, \mathrm{s}, \mathrm{CH}_{2} \mathrm{~N}$ ), 4.35 (major, $2 \mathrm{H}, \mathrm{d}, J=4.1 \mathrm{~Hz}$, $\mathrm{C}_{2} \mathrm{OH}$ ), 4.87 (minor, $2 \mathrm{H}, \mathrm{s}, \mathrm{C}_{2} \mathrm{~N}$ ), 6.53 (minor, $2 \mathrm{H}, \mathrm{d}, J=8.5 \mathrm{~Hz}, 2 \times$ ArH $) 6.55$ (major, $2 \mathrm{H}, \mathrm{d}, J=8.5 \mathrm{~Hz}, 2 \times \mathrm{Ar} \underline{\mathrm{H}}$ ) 7.20 (minor, $1 \mathrm{H}, \mathrm{t}, J=8.5 \mathrm{~Hz}, \operatorname{ArCHC} \underline{H C H}$ ), 7.24 (major, $1 \mathrm{H}, \mathrm{t}, J=8.5 \mathrm{~Hz}, \mathrm{ArCHCHCH}) ; \delta_{\mathrm{C}}\left(125 \mathrm{MHz}, \mathrm{CDCl}_{3}\right)$ major rotamer $19.5(2 \times$

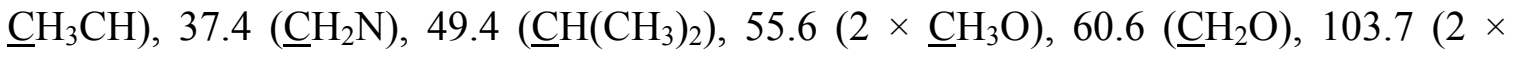
$\mathrm{Ar} \underline{\mathrm{C}} \mathrm{H}), 112.2(\mathrm{Ar} \underline{\mathrm{C}}), 129.6(\mathrm{Ar} \underline{\mathrm{CH}}), 159.0\left(2 \times \underline{\mathrm{C}}\left(\mathrm{OCH}_{3}\right)\right), 171.6(\mathrm{NC} \mathrm{O}) ; v_{\max }\left(\mathrm{CH}_{2} \mathrm{Cl}_{2}\right.$ evaporated film/ $\mathrm{cm}^{-1}$ ) 3400 (broad, OH), 2992, 2967 and $2842(\mathrm{CH}), 1643$ (amide C=O), $1595(\mathrm{C}=\mathrm{C}) ; \mathrm{m} / \mathrm{z}\left(\mathrm{ES}^{+}\right) 290(14 \%, \mathrm{M}+\mathrm{Na}), 210(8 \%) ; \mathrm{C}_{14} \mathrm{H}_{22} \mathrm{NO}_{4}\left(\mathrm{MH}^{+}\right)$found 268.1546, expected 268.1543; mp (iso-propanol) $64.1-65.2{ }^{\circ} \mathrm{C}$. 


\section{rac-2-iso-Propyl-6,10-dimethoxy-4-phenylsulfanyl-2-azaspiro-[4.5]-deca-6,9-diene-}

\section{3,8-dione 10}
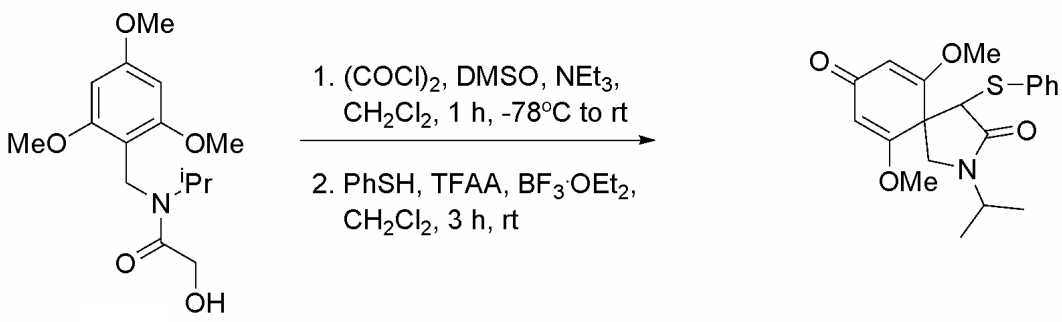

Following general procedure B: Treatment of the crude glyoxamide 2 (493 $\mathrm{mg}, 1.67$ mmol, 1 eq $)$ with thiophenol $(0.17 \mathrm{ml}, 1.67 \mathrm{mmol}, 1 \mathrm{eq})$, TFAA $(2.10 \mathrm{ml}, 15.0 \mathrm{mmol}, 9$ eq) and $\mathrm{BF}_{3} \cdot \mathrm{OEt}_{2}(1.04 \mathrm{ml}, 8.36 \mathrm{mmol}, 5 \mathrm{eq})$ gave a colourless oil. Purification by flash column chromatography on silica gel (40\%-80\% ethyl acetate/iso-hexane) afforded spirocycle 10 as a cream crystalline solid (412 mg, $1.11 \mathrm{mmol}, 66 \%$ over 2 steps).

$\delta_{\mathrm{H}}\left(400 \mathrm{MHz}, \mathrm{CDCl}_{3}\right) 1.16\left(6 \mathrm{H}, \mathrm{dd}, J=6.6 \mathrm{~Hz}, 2 \times \mathrm{CH}_{3} \mathrm{CH}\right), 3.37(1 \mathrm{H}, \mathrm{d}, J=9.7 \mathrm{~Hz}$, C $\left(1 \mathrm{H}\right.$, septet, $\left.J=6.6 \mathrm{~Hz}, \mathrm{C} \underline{\mathrm{H}}\left(\mathrm{CH}_{3}\right)_{2}\right), 4.58(1 \mathrm{H}, \mathrm{s}, \mathrm{C} \underline{\mathrm{HS}}), 5.28(1 \mathrm{H}, \mathrm{s}, \mathrm{C} \underline{\mathrm{HCOCH}}), 5.58(1 \mathrm{H}$, s, $\mathrm{CHCOC} \underline{\mathrm{H}}), 7.18-7.23(3 \mathrm{H}, \mathrm{m}, 3 \times \mathrm{Ar} \underline{\mathrm{H}}), 7.43-7.45(2 \mathrm{H}, \mathrm{m}, 2 \times \operatorname{Ar} \underline{\mathrm{H}}) ; \delta_{\mathrm{C}}(100 \mathrm{MHz}$, $\left.\mathrm{CDCl}_{3}\right) 18.9\left(\underline{\mathrm{CH}_{3} \mathrm{CH}}\right), 19.6\left(\underline{\mathrm{CH}}_{3} \mathrm{CH}\right), 43.7\left(\underline{\mathrm{CH}}\left(\mathrm{CH}_{3}\right)_{2}\right), 47.6\left(\underline{\mathrm{CH}}_{2} \mathrm{~N}\right), 51.0(\underline{\mathrm{C}}), 55.9$

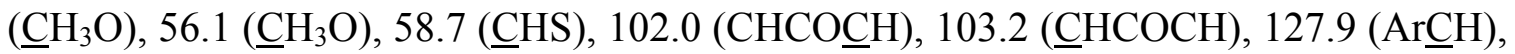
$128.8(2 \times \mathrm{Ar} \underline{\mathrm{CH}}), 133.1(2 \times \mathrm{ArC} H), 134.2(\mathrm{Ar} \underline{\mathrm{C}}), 168.4\left(\underline{\mathrm{C}}\left(\mathrm{OCH}_{3}\right)\right), 168.5\left(\underline{\mathrm{C}}\left(\mathrm{OCH}_{3}\right)\right)$, 172.4 (NCO), 187.1 (ㅁ); $v_{\max }\left(\mathrm{CH}_{2} \mathrm{Cl}_{2}\right.$ evaporated film/ $\left.\mathrm{cm}^{-1}\right) 2974$ and $2940(\mathrm{CH}), 1694$ $(\mathrm{C}=\mathrm{O}), 1658$ (lactam $\mathrm{C}=\mathrm{O}), 1628$ and $1598(\mathrm{C}=\mathrm{C}) ; \mathrm{m} / \mathrm{z}(\mathrm{ESI}) 396(\mathrm{M}+\mathrm{Na}, 8 \%), 374$ $\left(\mathrm{MH}^{+}, 100 \%\right) ; \mathrm{C}_{20} \mathrm{H}_{24} \mathrm{NO}_{4} \mathrm{~S}\left(\mathrm{MH}^{+}\right)$found 374.1425, expected 374.1421; mp $\left(\mathrm{CH}_{2} \mathrm{Cl}_{2}\right)$ 145.9-147. ${ }^{\circ} \mathrm{C}$. 


\section{rac-(1H,1H,2H,2H-Heptadecafluorodecylsulfanyl)-2-iso-propyl-6,10-dimethoxy-2-}

aza spiro-[4.5]-deca-6,9-diene-3,8-dione 11
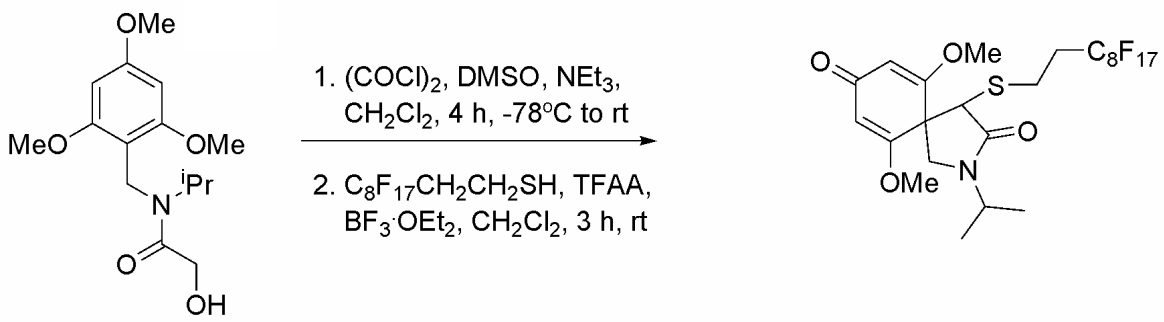

Following general procedure B: The crude glyoxamide $2(2.13 \mathrm{~g}, 7.23 \mathrm{mmol}, 1 \mathrm{eq})$ was treated with $1 \mathrm{H}, 1 \mathrm{H}, 2 \mathrm{H}, 2 \mathrm{H}$-perfluorodecane-1-thiol $(1.47 \mathrm{ml}, 5.04 \mathrm{mmol}, 0.7 \mathrm{eq}) \mathrm{TFAA}$ (9.11 ml, $65.1 \mathrm{mmol}, 9 \mathrm{eq})$ and $\mathrm{BF}_{3} \cdot \mathrm{OEt}_{2}(4.47 \mathrm{ml}, 36.0 \mathrm{mmol}, 5 \mathrm{eq})$. The reaction mixture was quenched and worked up with $1 \mathrm{M} \mathrm{NaOH}$ to give a yellow oil. Purification by fluorous solid phase extraction on FluoroFlash ${ }^{\circledR}$ silica gel (80\% acetonitrile/water, then $100 \%$ acetonitrile) gave spirocycle 11 as a yellow foam (2.75 g, 3.69 mmol, 73\% over 2 steps).

$\delta_{\mathrm{H}}\left(500 \mathrm{MHz}, \mathrm{CDCl}_{3}\right) 1.11\left(3 \mathrm{H}, \mathrm{d}, J=6.9 \mathrm{~Hz}, \mathrm{CH}_{3} \mathrm{CH}\right), 1.15(3 \mathrm{H}, \mathrm{d}, J=6.9 \mathrm{~Hz}$, $\left.\mathrm{C}_{3} \mathrm{CH}\right), 2.22-2.56\left(2 \mathrm{H}, \mathrm{m}, \underline{\mathrm{CH}}_{2} \mathrm{C}_{8} \mathrm{~F}_{17}\right), 2.77\left(2 \mathrm{H}, \mathrm{t}, J=8.2 \mathrm{~Hz}, \underline{\mathrm{C}}_{2} \mathrm{CH}_{2} \mathrm{C}_{8} \mathrm{~F}_{17}\right), 3.37(1 \mathrm{H}$, d, $J=9.8 \mathrm{~Hz}, \mathrm{C} \underline{H} H \mathrm{~N}), 3.64(1 \mathrm{H}, \mathrm{d}, J=9.8 \mathrm{~Hz}, \mathrm{CH} \underline{H N}), 3.67\left(3 \mathrm{H}, \mathrm{s}, \mathrm{CH}_{3} \mathrm{O}\right), 3.80(3 \mathrm{H}, \mathrm{s}$, $\left.\mathrm{C}_{3} \mathrm{O}\right), 4.06(1 \mathrm{H}, \mathrm{s}, \mathrm{C} \underline{\mathrm{HS}}), 4.43\left(1 \mathrm{H}\right.$, septet, $\left.J=6.9 \mathrm{~Hz}, \mathrm{C} \underline{H}\left(\mathrm{CH}_{3}\right)_{2}\right), 5.53(1 \mathrm{H}, \mathrm{s}$,

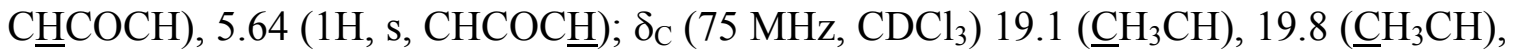

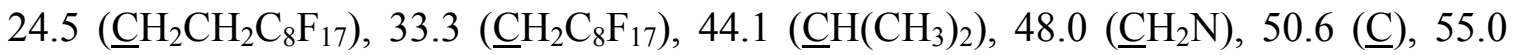
$(\underline{\mathrm{CHS}}), 56.1 \quad\left(\underline{\left.\mathrm{CH}_{3} \mathrm{O}\right)}, 56.6\left(\underline{\mathrm{CH}}_{3} \mathrm{O}\right), 102.1 \quad(\underline{\mathrm{CHCOCH}}), 103.8(\mathrm{CHCO} \underline{\mathrm{CH}}), 168.8\right.$ $\left(\underline{\mathrm{C}}\left(\mathrm{OCH}_{3}\right)\right), 169.2\left(\underline{\mathrm{C}}\left(\mathrm{OCH}_{3}\right)\right), 172.4(\mathrm{NCO}), 187.1$ ( $\left.\underline{\mathrm{CO}}\right) ; v_{\max }\left(\mathrm{CH}_{2} \mathrm{Cl}_{2}\right.$ evaporated film/ $\left.\mathrm{cm}^{-1}\right) 2977$ and $2943(\mathrm{CH}), 1631$ and $1599(\mathrm{C}=\mathrm{C}), 1695(\mathrm{C}=\mathrm{O}) 1659(\operatorname{lactam} \mathrm{C}=\mathrm{O})$; $\mathrm{m} / \mathrm{z}\left(\mathrm{ES}^{+}\right) 766(\mathrm{M}+\mathrm{Na}, 100 \%) ; \mathrm{C}_{24} \mathrm{H}_{22} \mathrm{NO}_{4} \mathrm{~F}_{17} \mathrm{SNa}(\mathrm{M}+\mathrm{Na})$ found 766.0895, expected 766.0890 . 
rac-4-Ethylsulfanyl-2-iso-propyl-6,10-dimethoxy-2-azaspiro-[4.5]-deca-6,9-diene3,8-dione 12
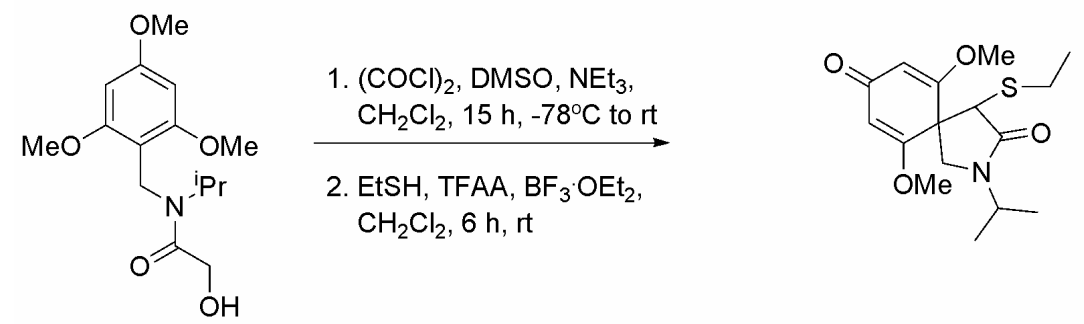

Following general procedure B: Treatment of the crude glyoxamide 2 (239 $\mathrm{mg}, 0.81$ mmol, 1 eq) with ethanethiol $(0.06 \mathrm{ml}, 0.81 \mathrm{mmol}, 1 \mathrm{eq})$, TFAA (1.03 ml, $7.29 \mathrm{mmol}, 9$ eq) and $\mathrm{BF}_{3} \cdot \mathrm{OEt}_{2}(0.51 \mathrm{ml}, 4.05 \mathrm{mmol}, 5 \mathrm{eq})$ gave a yellow oil. Removal of volatile impurities under high vacuum afforded spirocycle 12 as a yellow oil (229 mg, 0.70 mmol, $87 \%$ over 2 steps).

$\delta_{\mathrm{H}}\left(500 \mathrm{MHz}, \mathrm{CDCl}_{3}\right) 1.18\left(3 \mathrm{H}, \mathrm{d}, J=6.9 \mathrm{~Hz}, \mathrm{CH}_{3} \mathrm{CH}\right), 1.08-1.12\left(6 \mathrm{H}, \mathrm{m}, \mathrm{C}_{3} \mathrm{CH}\right.$ and $\left.\mathrm{C}_{3} \mathrm{CH}_{2}\right), 2.59\left(2 \mathrm{H}, \mathrm{qd}, J=7.6 \mathrm{~Hz}, 5.2 \mathrm{~Hz}, \underline{\mathrm{C}}_{2} \mathrm{CH}_{3}\right) 3.37(1 \mathrm{H}, \mathrm{d}, J=9.7 \mathrm{~Hz}, \mathrm{C} \underline{H} H \mathrm{~N})$, $3.62(1 \mathrm{H}, \mathrm{d}, J=9.7 \mathrm{~Hz}, \mathrm{CH} \underline{\mathrm{HN}}), 3.67\left(3 \mathrm{H}, \mathrm{s}, \mathrm{C}_{3} \mathrm{O}\right), 3.81\left(3 \mathrm{H}, \mathrm{s}, \mathrm{C}_{3} \mathrm{O}\right), 4.11(1 \mathrm{H}, \mathrm{s}$,

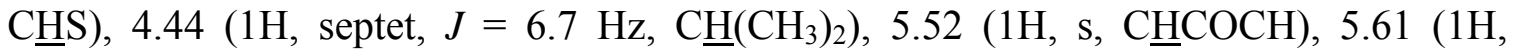
$\mathrm{CHCOC} \underline{\mathrm{H}}) ; \delta_{\mathrm{C}}\left(125 \mathrm{MHz}, \mathrm{CDCl}_{3}\right) 14.8\left(\underline{\mathrm{CH}}_{3} \mathrm{CH}_{2}\right), 18.9\left(\underline{\mathrm{C}} \mathrm{H}_{3} \mathrm{CH}\right), 19.6\left(\underline{\mathrm{CH}_{3} \mathrm{CH}}\right), 27.4$

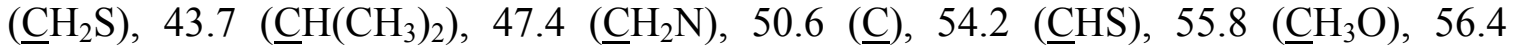
$\left(\underline{\mathrm{CH}_{3} \mathrm{O}}\right), 101.7(\mathrm{C} \underline{\mathrm{HCOCH}}), 103.3(\mathrm{CHCOC} \underline{\mathrm{H}}), 169.2\left(\underline{\mathrm{C}}\left(\mathrm{OCH}_{3}\right)\right), 169.6\left(\underline{\mathrm{C}}\left(\mathrm{OCH}_{3}\right)\right)$,

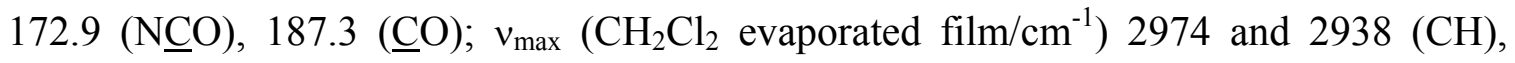
$1694(\mathrm{C}=\mathrm{O}), 1654(\operatorname{lactam} \mathrm{C}=\mathrm{O}), 1627$ and $1596(\mathrm{C}=\mathrm{C}) ; \mathrm{m} / \mathrm{z}\left(\mathrm{ES}^{+}\right) 326\left(\mathrm{MH}^{+}, 100 \%\right)$; $\mathrm{C}_{16} \mathrm{H}_{24} \mathrm{NO}_{4} \mathrm{~S}\left(\mathrm{MH}^{+}\right)$found 326.1424 , expected 326.1421. 


\section{rac-4-Hexylsulfanyl-2-iso-propyl-6,10-dimethoxy-2-azaspiro-[4.5]-deca-6,9-diene-}

\section{3,8-dione 13}
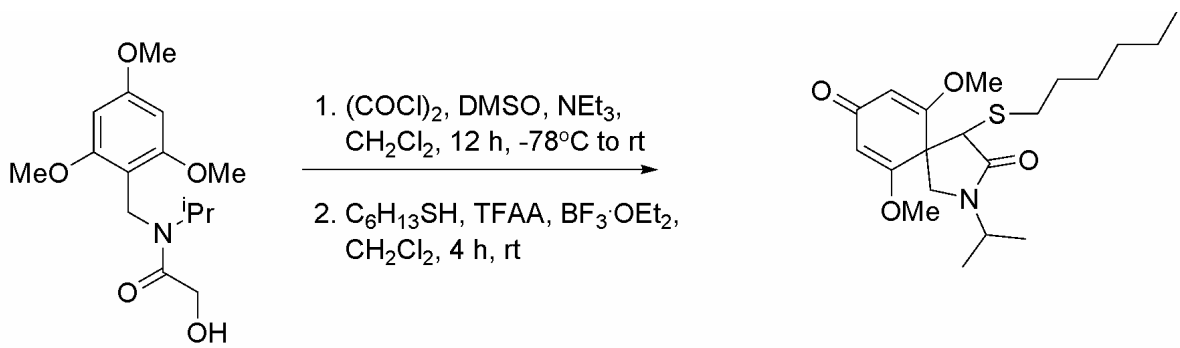

Following general procedure B: Treatment of the crude glyoxamide $2(0.70 \mathrm{~g}, 2.37$ mmol, 1 eq) with hexanethiol ( $0.33 \mathrm{ml}, 2.37 \mathrm{mmol}, 1$ eq), TFAA ( $3.02 \mathrm{ml}, 21.4 \mathrm{mmol}, 9$ eq) and $\mathrm{BF}_{3} \cdot \mathrm{OEt}_{2}(1.49 \mathrm{ml}, 11.9 \mathrm{mmol}, 5 \mathrm{eq})$ gave a brown oil. Removal of volatile impurities under high vacuum afforded spirocycle $\mathbf{1 3}$ as an orange oil (703 $\mathrm{mg}, 1.84$ mmol, $78 \%$ over 2 steps).

$\delta_{\mathrm{H}}\left(500 \mathrm{MHz}, \mathrm{CDCl}_{3}\right) 0.86\left(3 \mathrm{H}, \mathrm{t}, J=7.1 \mathrm{~Hz}, \mathrm{CH}_{2} \mathrm{C}_{3}\right), 1.13(3 \mathrm{H}, \mathrm{d}, J=6.9 \mathrm{~Hz}$, $\left.\mathrm{C}_{3} \mathrm{CH}\right), 1.16\left(3 \mathrm{H}, \mathrm{d}, J=6.9 \mathrm{~Hz}, \underline{\mathrm{CH}}_{3} \mathrm{CH}\right), 1.19-1.33\left(6 \mathrm{H}, \mathrm{m}, 3 \times \mathrm{C}_{2}\right), 1.43-1.51(2 \mathrm{H}$, $\left.\mathrm{m}, \mathrm{SCH}_{2} \mathrm{C}_{2} \mathrm{CH}_{2}\right), 2.57\left(2 \mathrm{H}, \mathrm{t}, J=7.4 \mathrm{~Hz}, \mathrm{SC}_{2} \mathrm{CH}_{2}\right), 3.37(1 \mathrm{H}, \mathrm{d}, J=9.8 \mathrm{~Hz}, \mathrm{C} \underline{H H N})$, $3.62(1 \mathrm{H}, \mathrm{d}, J=9.8 \mathrm{~Hz}, \mathrm{CH} \underline{\mathrm{HN}}), 3.68\left(3 \mathrm{H}, \mathrm{s}, \mathrm{C}_{3} \mathrm{O}\right), 3.81\left(3 \mathrm{H}, \mathrm{s}, \mathrm{C}_{3} \mathrm{O}\right), 4.10(1 \mathrm{H}, \mathrm{s}$, CㅌS), $4.45\left(1 \mathrm{H}\right.$, septet, $\left.J=6.9 \mathrm{~Hz}, \mathrm{C} \underline{\mathrm{H}}\left(\mathrm{CH}_{3}\right)_{2}\right), 5.53(1 \mathrm{H}, \mathrm{s}, \mathrm{C} \underline{\mathrm{HCOCH}}), 5.63(1 \mathrm{H}, \mathrm{s}$, $\mathrm{CHCOC} \underline{\mathrm{H}}) ; \delta_{\mathrm{C}}\left(125 \mathrm{MHz}, \mathrm{CDCl}_{3}\right) 14.0\left(\underline{\mathrm{CH}}_{3} \mathrm{CH}_{2}\right), 18.9\left(\underline{\mathrm{CH}}_{3} \mathrm{CH}\right), 19.6\left(\underline{\mathrm{C}} \mathrm{H}_{3} \mathrm{CH}\right), 22.5$ $\left(\underline{\mathrm{CH}}_{2}\right), 28.3\left(\underline{\mathrm{CH}}_{2}\right), 29.8\left(\mathrm{SCH}_{2} \underline{\mathrm{CH}}_{2}\right), 31.4\left(\underline{\mathrm{CH}}_{2}\right), 33.5\left(\mathrm{~S}_{\mathrm{CH}} \mathrm{CH}_{2}\right), 43.6\left(\underline{\mathrm{CH}}\left(\mathrm{CH}_{3}\right)_{2}\right)$, $47.4\left(\underline{\mathrm{CH}}_{2} \mathrm{~N}\right), 50.6(\underline{\mathrm{C}}), 54.5(\underline{\mathrm{C}} \mathrm{HS}), 55.8\left(\underline{\mathrm{CH}}_{3} \mathrm{O}\right), 56.3\left(\underline{\mathrm{CH}}_{3} \mathrm{O}\right), 102.7(\underline{\mathrm{CHCOCH}}), 103.3$ $(\mathrm{CHCO} \underline{\mathrm{CH}}), 169.2\left(\underline{\mathrm{C}}\left(\mathrm{OCH}_{3}\right)\right), 169.7\left(\underline{\mathrm{C}}\left(\mathrm{OCH}_{3}\right)\right), 172.9(\mathrm{~N} \underline{\mathrm{CO}}), 187.3(\underline{\mathrm{CO}}) ; v_{\max }$ (neat $\left./ \mathrm{cm}^{-1}\right) 2930$ and $2855(\mathrm{CH}), 1695(\mathrm{C}=\mathrm{O}) 1659($ lactam $\mathrm{C}=\mathrm{O}), 1631$ and $1597(\mathrm{C}=\mathrm{C})$; $\mathrm{m} / \mathrm{z}\left(\mathrm{ES}^{+}\right) 404(\mathrm{M}+\mathrm{Na}, 100 \%), 382\left(\mathrm{MH}^{+}, 72 \%\right) ; \mathrm{C}_{20} \mathrm{H}_{31} \mathrm{NO}_{4} \mathrm{SNa}(\mathrm{M}+\mathrm{Na})$ found 404.1861, expected 404.1866 . 


\section{rac-2-iso-Propyl-4-iso-propylsulfanyl-6,10-dimethoxy-2-azaspiro-[4.5]-deca-6,9-}

\section{diene-3,8-dione 14}
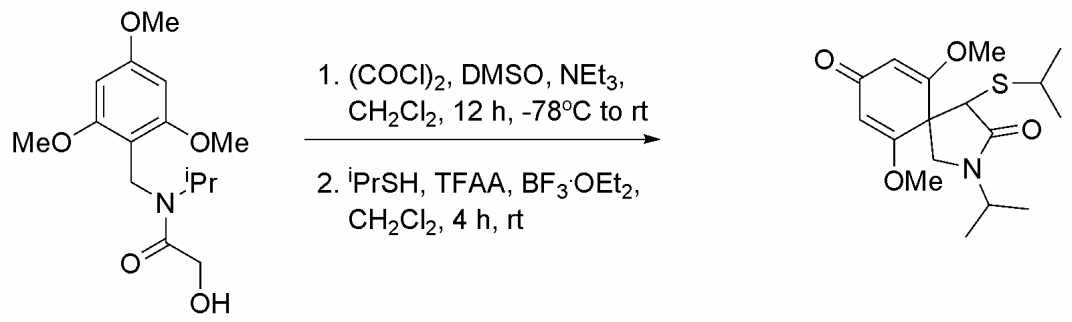

Following general procedure B: Treatment of the crude glyoxamide 2 (460 $\mathrm{mg}, 1.56$ mmol, 1 eq) with 2-propanethiol ( $0.15 \mathrm{ml}, 1.56 \mathrm{mmol}, 1 \mathrm{eq})$, TFAA (1.98 ml, $14.0 \mathrm{mmol}$, 9 eq) and $\mathrm{BF}_{3} \cdot \mathrm{OEt}_{2}(0.98 \mathrm{ml}, 7.80 \mathrm{mmol}, 5 \mathrm{eq})$ gave a brown oil. Removal of volatile impurities under high vacuum afforded spirocycle 14 a yellow oil (495 mg, 1.46 mmol, 94\% over 2 steps).

$\delta_{\mathrm{H}}\left(500 \mathrm{MHz}, \mathrm{CDCl}_{3}\right) 1.13\left(3 \mathrm{H}, \mathrm{d}, J=6.6 \mathrm{~Hz}, \underline{\mathrm{CH}}_{3} \mathrm{CH}\right), 1.15(3 \mathrm{H}, \mathrm{d}, J=6.6 \mathrm{~Hz}$, $\left.\mathrm{C}_{3} \mathrm{CH}\right), 1.17\left(3 \mathrm{H}, \mathrm{d}, J=6.6 \mathrm{~Hz}, \mathrm{C}_{3} \mathrm{CH}\right), 1.22\left(3 \mathrm{H}, \mathrm{d}, J=6.6 \mathrm{~Hz}, \underline{\mathrm{C}}_{3} \mathrm{CH}\right), 3.01(1 \mathrm{H}$, septet, $\left.J=6.6 \mathrm{~Hz}, \mathrm{SC} \underline{\mathrm{H}}\left(\mathrm{CH}_{3}\right)_{2}\right), 3.36(1 \mathrm{H}, \mathrm{d}, J=9.6 \mathrm{~Hz}, \mathrm{C} \underline{H} H \mathrm{~N}), 3.64(1 \mathrm{H}, \mathrm{d}, J=9.6 \mathrm{~Hz}$,

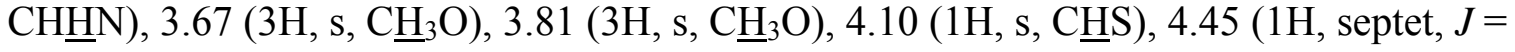
$\left.6.6 \mathrm{~Hz}, \mathrm{NC} \underline{\mathrm{H}}\left(\mathrm{CH}_{3}\right)_{2}\right), 5.52(1 \mathrm{H}, \mathrm{s}, \mathrm{C} \underline{\mathrm{HCOCH}}), 5.64(1 \mathrm{H}, \mathrm{s}, \mathrm{CHCOC} \underline{\mathrm{H}}) ; \delta_{\mathrm{C}}(125 \mathrm{MHz}$, $\left.\mathrm{CDCl}_{3}\right) 18.9\left(\underline{\mathrm{CH}}_{3} \mathrm{CH}\right), 19.5\left(\underline{\mathrm{CH}}_{3} \mathrm{CH}\right), 23.1\left(\underline{\mathrm{CH}}_{3} \mathrm{CH}\right), 23.6\left(\underline{\mathrm{CH}}_{3} \mathrm{CH}\right), 36.1\left(\left(\mathrm{CH}_{3}\right)_{2} \underline{\mathrm{CHS}}\right)$, $43.8\left(\left(\mathrm{CH}_{3}\right)_{2} \underline{\mathrm{C}} \mathrm{HN}\right), 47.4\left(\underline{\mathrm{CH}}_{2} \mathrm{~N}\right), 50.6(\underline{\mathrm{C}}), 52.6(\underline{\mathrm{CHS}}), 55.8\left(\underline{\mathrm{CH}}_{3} \mathrm{O}\right), 56.3\left(\underline{\mathrm{CH}}_{3} \mathrm{O}\right)$, $101.6(\underline{\mathrm{C} H C O C H}), 103.5(\mathrm{CHCO} \underline{\mathrm{H}}), 169.1\left(\underline{\mathrm{C}}\left(\mathrm{OCH}_{3}\right)\right), 169.9\left(\underline{\mathrm{C}}\left(\mathrm{OCH}_{3}\right)\right), 173.1$ (NCO), 187.5 (ㅁ); $v_{\max } 2974$ and $2941(\mathrm{CH}), 1694(\mathrm{C}=\mathrm{O}), 1656$ (lactam $\left.\mathrm{C}=\mathrm{O}\right), 1625$ and $1598(\mathrm{C}=\mathrm{C}) ; \mathrm{m} / \mathrm{z}\left(\mathrm{ES}^{+}\right) 362(\mathrm{M}+\mathrm{Na}, 100 \%), 340\left(\mathrm{MH}^{+}, 17 \%\right), 133(42 \%)$; $\mathrm{C}_{17} \mathrm{H}_{25} \mathrm{NO}_{4} \mathrm{SNa}(\mathrm{M}+\mathrm{Na})$ found 362.1390, expected 362.1397. 
rac-4-Cyclohexylsulfanyl-2-iso-propyl-6,10-dimethoxy-2-azaspiro-[4.5]-deca-6,9diene-3,8-dione 15
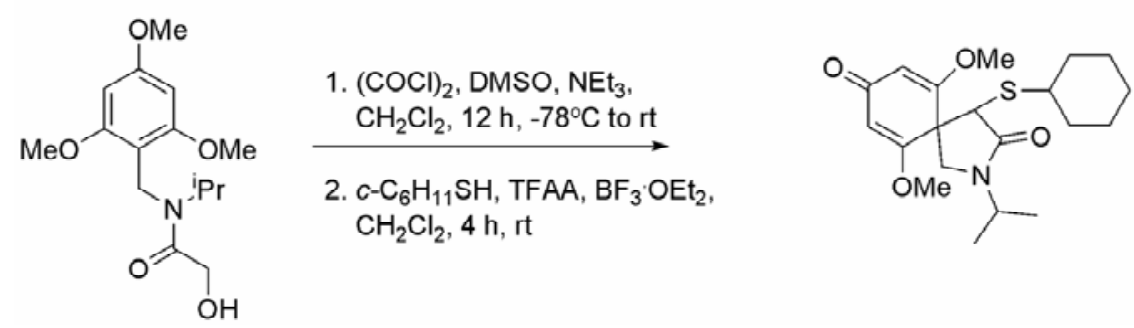

Following general procedure B: Treatment of the crude glyoxamide $2(0.50 \mathrm{~g}, 1.69$ mmol, 1 eq) with cyclohexanethiol $(0.21 \mathrm{ml}, 1.69 \mathrm{mmol}, 1 \mathrm{eq})$, TFAA (2.15 ml, 15.3 mmol, 9 eq $)$ and $\mathrm{BF}_{3} \cdot \mathrm{OEt}_{2}(1.06 \mathrm{ml}, 8.47 \mathrm{mmol}, 5 \mathrm{eq})$ gave a brown oil. Removal of volatile impurities under high vacuum afforded spirocycle 15 as an orange oil (566 mg, $1.49 \mathrm{mmol}, 88 \%$ over 2 steps).

$\delta_{\mathrm{H}}\left(500 \mathrm{MHz}, \mathrm{CDCl}_{3}\right) 1.05\left(3 \mathrm{H}, \mathrm{d}, J=6.7 \mathrm{~Hz}, \underline{\mathrm{CH}}_{3} \mathrm{CH}\right), 1.09(3 \mathrm{H}, \mathrm{d}, J=6.7 \mathrm{~Hz}$, $\left.\mathrm{C}_{3} \mathrm{CH}\right), 1.10-1.20(5 \mathrm{H}, \mathrm{m}, 5 \times \mathrm{CH}$ in cyclohexyl ring), 1.46-1.52 $(1 \mathrm{H}, \mathrm{m}, \mathrm{CH}$ in cyclohexyl ring), 1.60-1.73 $(3 \mathrm{H}, \mathrm{m}, 3 \times \mathrm{C} \underline{\mathrm{H}}$ in cyclohexyl ring), $1.84-1.92(1 \mathrm{H}, \mathrm{m}, \mathrm{C} \underline{\mathrm{H}}$ in cyclohexyl ring), 2.60-2.69 $\left(1 \mathrm{H}, \mathrm{m}, \mathrm{SC} \underline{\mathrm{H}}\left(\mathrm{CH}_{2}\right)_{2}\right), 3.30(1 \mathrm{H}, \mathrm{d}, J=9.8 \mathrm{~Hz}, \mathrm{C} \underline{\mathrm{H}} \mathrm{HN}), 3.57$ $(1 \mathrm{H}, \mathrm{d}, J=9.8 \mathrm{~Hz}, \mathrm{CH} \underline{\mathrm{HN}}), 3.61\left(3 \mathrm{H}, \mathrm{s}, \mathrm{C}_{3} \mathrm{O}\right), 3.74\left(3 \mathrm{H}, \mathrm{s}, \underline{\mathrm{C}}_{3} \mathrm{O}\right), 4.05$ (1H, s, C $\left.\mathrm{H}\right)$, $4.38\left(1 \mathrm{H}\right.$, septet, $\left.J=6.7 \mathrm{~Hz}, \underline{\mathrm{CH}}\left(\mathrm{CH}_{3}\right)_{2}\right), 5.47(1 \mathrm{H}, \mathrm{s}, \underline{\mathrm{CHCOCH}}), 5.60(1 \mathrm{H}, \mathrm{s}$, $\mathrm{CHCOC} \underline{\mathrm{H}}) ; \delta_{\mathrm{C}}\left(75 \mathrm{MHz}, \mathrm{CDCl}_{3}\right) 19.1\left(\underline{\mathrm{CH}}_{3} \mathrm{CH}\right), 19.7\left(\underline{\mathrm{C}} \mathrm{H}_{3} \mathrm{CH}\right), 25.8\left(\underline{\mathrm{CH}}_{2}\right), 26.1\left(\underline{\mathrm{CH}}_{2}\right)$, $26.2\left(\underline{\mathrm{CH}}_{2}\right), 33.4\left(\underline{\mathrm{CH}}_{2}\right), 34.0\left(\underline{\mathrm{CH}}_{2}\right), 44.1\left(\underline{\mathrm{CH}}\left(\mathrm{CH}_{3}\right)_{2}\right), 44.8\left(\mathrm{~S} \underline{\mathrm{CH}}\left(\mathrm{CH}_{2}\right)_{2}\right), 47.7\left(\underline{\mathrm{CH}}_{2} \mathrm{~N}\right)$,

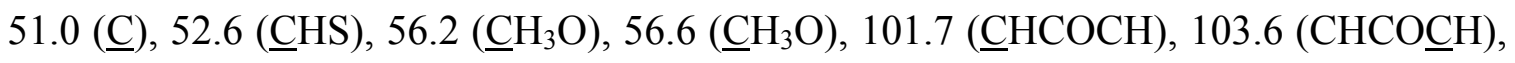
$169.6\left(\underline{\mathrm{C}}\left(\mathrm{OCH}_{3}\right)\right), 170.4\left(\underline{\mathrm{C}}\left(\mathrm{OCH}_{3}\right)\right), 173.6(\mathrm{NCO}), 188.0(\underline{\mathrm{CO}}) ; v_{\max }\left(\right.$ neat $\left./ \mathrm{cm}^{-1}\right) 2974$ and 2932 and $2852(\mathrm{CH}), 1695(\mathrm{C}=\mathrm{O}) 1656($ lactam $\mathrm{C}=\mathrm{O}), 1630$ and $1598(\mathrm{C}=\mathrm{C}) ; \mathrm{m} / \mathrm{z}\left(\mathrm{ES}^{+}\right)$ $402(\mathrm{M}+\mathrm{Na}, 36 \%), 380\left(\mathrm{MH}^{+}, 20 \%\right) ; \mathrm{C}_{20} \mathrm{H}_{29} \mathrm{NO}_{4} \mathrm{SNa}(\mathrm{M}+\mathrm{Na})$ found 402.1716, expected 402.1710 . 
rac-4-(1H,1H,2,H,2H-Heptadecafluorodecylsulfanyl)-6,10-dimethoxy-2-(2phenylsulfonyl ethyl)-2-aza spiro-[4.5]-deca-6,9-diene-3,8-dione 16
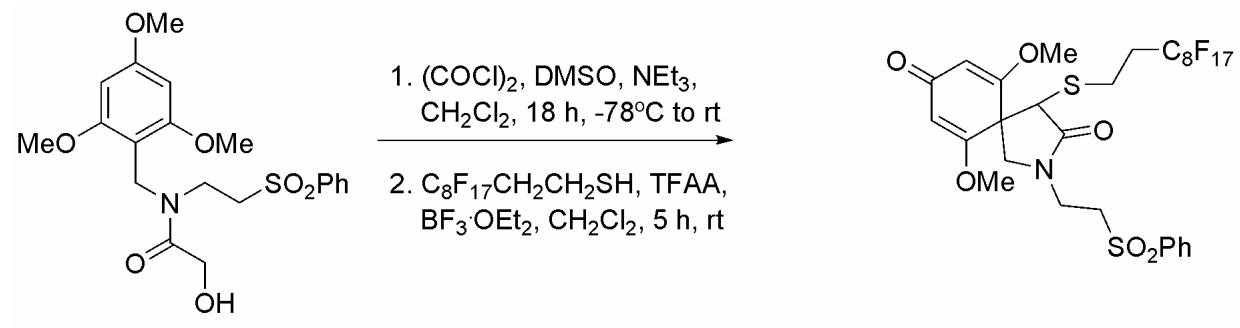

Following general procedure B: Treatment of the crude glyoxamide 3 (320 mg, 0.76 mmol, $1 \mathrm{eq})$ with $1 \mathrm{H}, 1 \mathrm{H}, 2 \mathrm{H}, 2 \mathrm{H}-$ perfluorodecane-1-thiol $(0.16 \mathrm{ml}, 0.53 \mathrm{mmol}, 0.7 \mathrm{eq})$, TFAA $(0.97 \mathrm{ml}, 6.84 \mathrm{mmol}, 9 \mathrm{eq})$ and $\mathrm{BF}_{3} \cdot \mathrm{OEt}_{2}(0.48 \mathrm{ml}, 3.80 \mathrm{mmol}, 5 \mathrm{eq})$ gave a yellow solid. Purification by fluorous solid phase extraction on FluoroFlash ${ }^{\circledR}$ silica gel (80\% methanol/water-100\% methanol) removed non-fluorous impurities then flash column chromatography on silica gel ( $80 \%$ ethyl acetate/petroleum ether) afforded spirocycle 16 as a yellow foam (237 mg, $0.27 \mathrm{mmol}, 51 \%$ over two steps).

$\delta_{\mathrm{H}}\left(500 \mathrm{MHz}, \mathrm{CDCl}_{3}\right)$ 2.20-2.51 (2H, m, $\left.\mathrm{C}_{2} \mathrm{C}_{8} \mathrm{~F}_{17}\right), 2.74-2.82\left(2 \mathrm{H}, \mathrm{m}, \mathrm{C}_{2} \mathrm{CH}_{2} \mathrm{C}_{8} \mathrm{~F}_{17}\right)$, 3.26-3.33 (1H, m, $\left.\underline{H H}_{2}\right)$, 3.45-3.54 (1H, m, $\left.\underline{\mathrm{HHCH}}_{2} \mathrm{SO}_{2}\right), 3.50(1 \mathrm{H}, \mathrm{d}, J=9.8 \mathrm{~Hz}$,

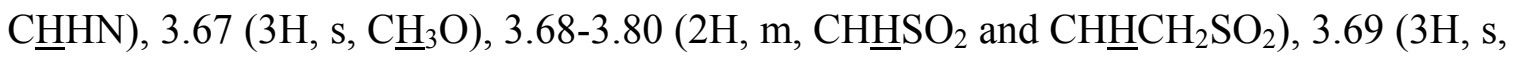

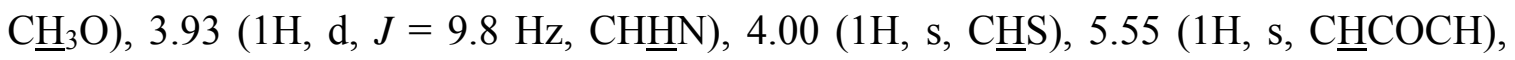
$5.65(1 \mathrm{H}, \mathrm{s}, \mathrm{CHCOC} \underline{\mathrm{H}}), 7.61(2 \mathrm{H}, \mathrm{m}, 2 \times \mathrm{Ar} \underline{\mathrm{H}}), 7.70(1 \mathrm{H}, \mathrm{tt}, J=7.6 \mathrm{~Hz}, 1.3 \mathrm{~Hz}, \mathrm{Ar} \underline{\mathrm{H}})$, $7.92(2 \mathrm{H}, \mathrm{d}, J=8.2 \mathrm{~Hz}, 2 \times \mathrm{Ar} \underline{\mathrm{H}}) ; \delta_{\mathrm{C}}\left(75 \mathrm{MHz}, \mathrm{CDCl}_{3}\right) 24.5\left(\underline{\mathrm{CH}}_{2} \mathrm{CH}_{2} \mathrm{C}_{8} \mathrm{~F}_{17}\right), 32.9$ $\left(\underline{\mathrm{CH}}_{2} \mathrm{C}_{8} \mathrm{~F}_{17}\right), 37.9\left(\underline{\mathrm{CH}}_{2}\right), 50.9(\underline{\mathrm{C}}), 53.1\left(\underline{\mathrm{CH}}_{2}\right), 53.4\left(\underline{\mathrm{CH}}_{2}\right), 54.1(\underline{\mathrm{C}} \mathrm{HS}), 56.5\left(\underline{\mathrm{CH}}_{3} \mathrm{O}\right)$, $56.7\left(\underline{\left.\mathrm{CH}_{3} \mathrm{O}\right),} 102.5(\underline{\mathrm{CHCOCH}}), 104.0(\mathrm{CHCO} \underline{\mathrm{H}}), 128.2(2 \times \mathrm{Ar} \underline{\mathrm{CH}}), 129.8(2 \times\right.$

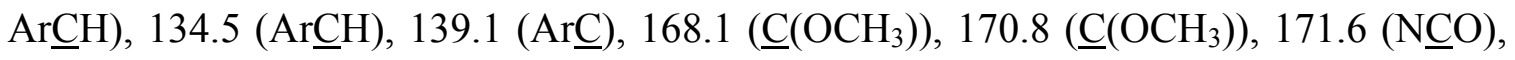
$186.8(\underline{\mathrm{CO}}) ; v_{\max }\left(\mathrm{CH}_{2} \mathrm{Cl}_{2}\right.$ evaporated film/ $\left.\mathrm{cm}^{-1}\right)$ 3061, 2977, 2942 and $2849(\mathrm{CH}), 1703$ $(\mathrm{C}=\mathrm{O}) 1651(\operatorname{lactam} \mathrm{C}=\mathrm{O}), 1598(\mathrm{C}=\mathrm{C}) ; \mathrm{m} / \mathrm{z}\left(\mathrm{ES}^{+}\right) 887\left(\mathrm{M}+\mathrm{NH}_{4}^{+}, 100 \%\right), 870\left(\mathrm{MH}^{+}\right.$, $9 \%) ; \mathrm{C}_{29} \mathrm{H}_{28} \mathrm{~N}_{2} \mathrm{O}_{6} \mathrm{~F}_{17} \mathrm{~S}_{2}\left(\mathrm{M}+\mathrm{NH}_{4}{ }^{+}\right)$found 887.1090, expected 887.1112. 
rac- $(4 R, 5 R) \quad$ and $\quad$ rac- $(4 R, 5 S)-4-(1 \mathrm{H}, 1 \mathrm{H}, 2 \mathrm{H}, 2 \mathrm{H}-\mathrm{Heptadecafluorodecylsulfanyl)-6-}$ methoxy-2-(2-phenylsulfonylethyl)-2-aza spiro-[4.5]-deca-6,9-diene-3,8-dione 17
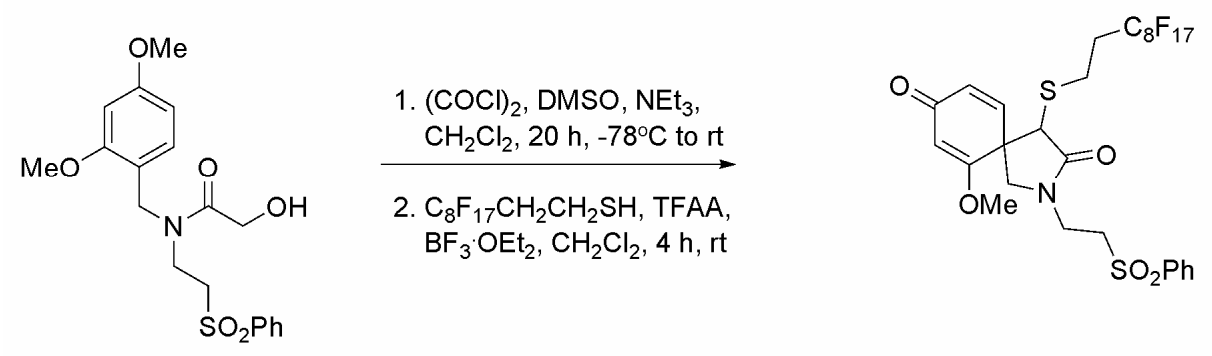

Following general procedure B: Treatment of the crude glyoxamide 4 (340 $\mathrm{mg}, 0.87$ mmol, 1 eq) with $1 \mathrm{H}, 1 \mathrm{H}, 2 \mathrm{H}, 2 \mathrm{H}$-perfluorodecane-1-thiol $(0.18 \mathrm{ml}, 0.61 \mathrm{mmol}, 0.7 \mathrm{eq})$, TFAA (1.11 ml, $7.83 \mathrm{mmol}, 9 \mathrm{eq})$ and $\mathrm{BF}_{3} \cdot \mathrm{OEt}_{2}(0.55 \mathrm{ml}, 4.35 \mathrm{mmol}, 5 \mathrm{eq})$ gave a 1:1 mixture of diastereoisomers, as a yellow solid. Purification by flash column chromatography on silica gel ( $80 \%$ ethyl acetate/petroleum ether) gave spirocycle $\mathbf{1 7}$ as a 1:1 mixture of diastereoisomers, as a yellow foam $(350 \mathrm{mg}, 0.42 \mathrm{mmol}, 69 \%$ over 2 steps).

For a 1:1 mixture of diasteroisomers: $\delta_{\mathrm{H}}\left(500 \mathrm{MHz}, \mathrm{CDCl}_{3}\right)$ 2.06-2.32 (both diastereoisomers, $4 \mathrm{H}, \mathrm{m}, 2 \times \mathrm{C}_{2} \mathrm{C}_{8} \mathrm{~F}_{17}$ ), 2.60-2.67 (both diastereoisomers, $4 \mathrm{H}, \mathrm{m}, 2 \times$ $\mathrm{C}_{2} \mathrm{CH}_{2} \mathrm{C}_{8} \mathrm{~F}_{17}$ ), 2.99-3.70 (both diastereoisomers, $8 \mathrm{H}, \mathrm{m}, 2 \times \mathrm{C}_{2} \mathrm{SO}_{2}$ and $2 \times$ $\underline{\mathrm{C}}_{2} \mathrm{CH}_{2} \mathrm{SO}_{2}$ ), 3.19 (one diastereoisomer, $1 \mathrm{H}, \mathrm{d}, J=9.4 \mathrm{~Hz}, \mathrm{C} \underline{\mathrm{HHN}}$ ), 3.34 (one diastereoisomer, $1 \mathrm{H}, \mathrm{s}, \mathrm{C} \underline{\mathrm{HS}}$ ), 3.36 (one diastereoisomer, $1 \mathrm{H}, \mathrm{d}, J=10.2 \mathrm{~Hz}, \mathrm{C} \underline{H} H \mathrm{~N}$ ), 3.49 (one diastereoisomer, $3 \mathrm{H}, \mathrm{s}, \mathrm{C}_{3} \mathrm{O}$ ), 3.54 (one diastereoisomer, $1 \mathrm{H}, \mathrm{d}, J=10.2 \mathrm{~Hz}$, $\mathrm{CH} \underline{\mathrm{HN}}$ ), 3.61 (one diastereoisomer, $3 \mathrm{H}, \mathrm{s}, \mathrm{CH}_{3} \mathrm{O}$ ), 3.75 (one diastereoisomer, $1 \mathrm{H}, \mathrm{d}, J=$ $9.4 \mathrm{~Hz}, \mathrm{CH} \underline{\mathrm{HN}}$ ), 3.84 (one diastereoisomer, 1H, s, CㅌS), 5.42 (one diastereoisomer, 1H, $\mathrm{s},\left(\mathrm{CH}_{3} \mathrm{O}\right) \mathrm{CC} \underline{\mathrm{HCO}}$ ), 5.56 (one diastereoisomer, $1 \mathrm{H}, \mathrm{s},\left(\mathrm{CH}_{3} \mathrm{O}\right) \mathrm{CC} \underline{\mathrm{HCO}}$ ), 6.10 (one diastereoisomer, $1 \mathrm{H}, \mathrm{d}, J=9.8 \mathrm{~Hz}, \underline{\mathrm{HCH}}$ ), 6.11 (one diastereoisomer, $1 \mathrm{H}, \mathrm{d}, J=9.8 \mathrm{~Hz}$, $\mathrm{C} \underline{\mathrm{HCH}}$ ), $6.41(1 \mathrm{H}, \mathrm{d}, J=9.8 \mathrm{~Hz}, \mathrm{CHC} \underline{\mathrm{H}}$ ), 6.54 (one diastereoisomer, $1 \mathrm{H}, \mathrm{d}, J=9.8 \mathrm{~Hz}$, $\mathrm{CHC} \underline{\mathrm{H}}$ ), 7.39 (both diastereoisomers, $4 \mathrm{H}$, apparent td, $J=7.5 \mathrm{~Hz}, J=1.7 \mathrm{~Hz}, 4 \times \mathrm{Ar} \underline{\mathrm{H}}$ ), 7.46-7.51 (both diastereoisomers, $2 \mathrm{H}, \mathrm{m}, 2 \times \mathrm{Ar} \underline{\mathrm{H}}$ ), 7.70-7.74 (both diastereoisomers, $4 \mathrm{H}$,

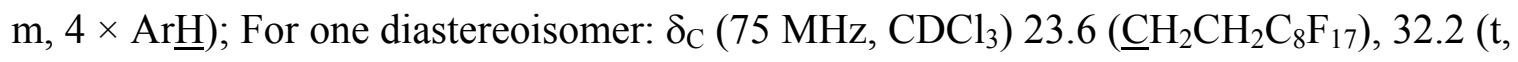
$\left.J^{2}{ }_{\mathrm{CF}}=23 \mathrm{~Hz}, \underline{\mathrm{C}} \mathrm{H}_{2} \mathrm{C}_{8} \mathrm{~F}_{17}\right), 37.9\left(\underline{\mathrm{CH}}_{2} \mathrm{CH}_{2} \mathrm{SO}_{2}\right), 48.8(\underline{\mathrm{C}}), 53.0\left(\underline{\mathrm{CH}_{2} \mathrm{~N}}\right), 53.1$ (ㄷS), 53.4 
$\left(\underline{\mathrm{C}} \mathrm{H}_{2} \mathrm{SO}_{2}\right), 56.3\left(\underline{\mathrm{CH}} \mathrm{H}_{3} \mathrm{O}\right), 105.4(\underline{\mathrm{CH}}), 128.2(2 \times \mathrm{Ar} \underline{\mathrm{CH}}), 129.7(2 \times \mathrm{Ar} \underline{\mathrm{CH}}), 130.6(\underline{\mathrm{CH}})$, $134.5(\mathrm{Ar} \underline{\mathrm{C}} \mathrm{H}), 139.0(\underline{\mathrm{C}}), 143.3(\underline{\mathrm{CH}}), 170.7\left(\underline{\mathrm{C}}\left(\mathrm{OCH}_{3}\right)\right), 174.0(\mathrm{~N} \underline{\mathrm{CO}}), 186.6(\underline{\mathrm{CO}}) ; v_{\max }$ $\left(\mathrm{CH}_{2} \mathrm{Cl}_{2}\right.$ evaporated film $\left./ \mathrm{cm}^{-1}\right)$ 3062, 2928 and $2852(\mathrm{CH}), 1698(\mathrm{C}=\mathrm{O}), 1660$ (lactam $\mathrm{C}=\mathrm{O}), 1633$ and $1599(\mathrm{C}=\mathrm{C}) ; v_{\max }\left(\mathrm{ES}^{+}\right) 839\left(\mathrm{M}^{+}, 100 \%\right) ; \mathrm{C}_{28} \mathrm{H}_{23} \mathrm{O}_{5} \mathrm{NF}_{17} \mathrm{~S}_{2}\left(\mathrm{MH}^{+}\right)$found 840.0741, expected 840.0744.

rac-(4R,5R) and rac-(4R,5S)-4-(1H,1H,2H,2H-Heptadecafluorodecylsulfanyl)-6methoxy-2-tert-butyl-2-aza spiro-[4.5]-deca-6,9-diene-3,8-dione 18
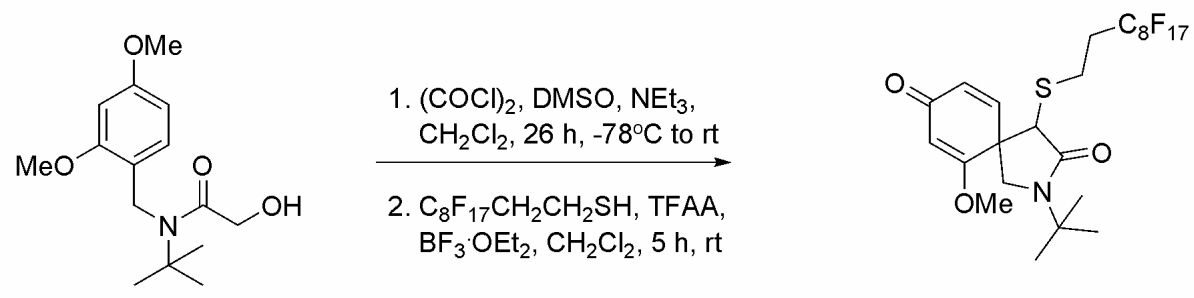

Following general procedure B: Treatment of the crude glyoxamide $5(88 \mathrm{mg}, 0.32$ mmol, 1 eq) with $1 \mathrm{H}, 1 \mathrm{H}, 2 \mathrm{H}, 2 \mathrm{H}$-perfluorodecane-1-thiol $(0.06 \mathrm{ml}, 0.22 \mathrm{mmol}, 0.7 \mathrm{eq})$ TFAA (0.40 ml, $2.84 \mathrm{mmol}, 9 \mathrm{eq})$ and $\mathrm{BF}_{3} \cdot \mathrm{OEt}_{2}(0.20 \mathrm{ml}, 1.58 \mathrm{mmol}, 5 \mathrm{eq})$ gave a 2:1 mixture of diastereoisomers, as a yellow solid. Purification by flash column chromatography on silica gel (50\% ethyl acetate/petroleum ether) afforded spirocycle $\mathbf{1 8}$ as a 2:1 mixture of diastereoisomers, as a yellow foam $(86 \mathrm{mg}, 0.18 \mathrm{mmol}, 54 \%$ over 2 steps).

For a 2:1 mixture of diasteroisomers: $\delta_{\mathrm{H}}\left(500 \mathrm{MHz}, \mathrm{CDCl}_{3}\right) 1.43$ (minor, $9 \mathrm{H}, \mathrm{s}, 3 \times$ $\mathrm{C}_{3} \mathrm{C}$ ), 1.44 (major, 9H, s, $3 \times \mathrm{C}_{3} \mathrm{C}$ ), 2.26-2.53 (major, $2 \mathrm{H}, \mathrm{m}, \mathrm{C}_{2} \mathrm{C}_{8} \mathrm{~F}_{17}$ and minor, $2 \mathrm{H}, \mathrm{m}, \mathrm{C}_{2} \mathrm{C}_{8} \mathrm{~F}_{17}$ ), 2.80-2.93 (major, $2 \mathrm{H}, \mathrm{m}, \mathrm{C}_{2} \mathrm{CH}_{2} \mathrm{C}_{8} \mathrm{~F}_{17}$ and minor, $2 \mathrm{H}, \mathrm{m}$, $\mathrm{C}_{2} \mathrm{CH}_{2} \mathrm{C}_{8} \mathrm{~F}_{17}$ ), 3.29 (major, $1 \mathrm{H}, \mathrm{d}, J=9.6 \mathrm{~Hz}, \mathrm{CH} H \mathrm{H}$ ), 3.54 (minor, $1 \mathrm{H}, \mathrm{d}, J=10.4 \mathrm{~Hz}$,

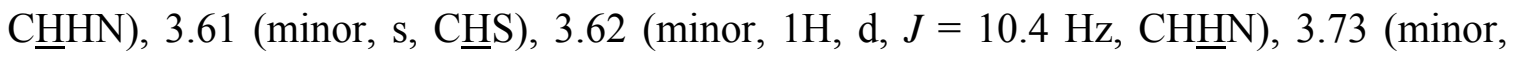
$3 \mathrm{H}, \mathrm{s}, \mathrm{C}_{3} \mathrm{O}$ ), 3.79 (major, $1 \mathrm{H}, \mathrm{d}, J=9.6 \mathrm{~Hz}, \mathrm{CH} \underline{\mathrm{HN}}$ ), 3.83 (major, $3 \mathrm{H}, \mathrm{s}, \mathrm{C}_{3} \mathrm{O}$ ), 4.06 (major, 1H, s, CㅌS), 5.63 (minor, $1 \mathrm{H}, \mathrm{d}, J=1.6 \mathrm{~Hz}, \mathrm{C} \underline{\mathrm{HCOCH}}=\mathrm{CH}$ ), 5.77 (major, $1 \mathrm{H}, \mathrm{d}$, $J=1.3 \mathrm{~Hz}, \mathrm{C} \underline{\mathrm{HCOCH}}=\mathrm{CH}$ ), 6.32 (minor, $1 \mathrm{H}, \mathrm{dd}, J=9.8 \mathrm{~Hz}, 1.6 \mathrm{~Hz}, \mathrm{COC} \underline{\mathrm{H}}=\mathrm{CH}$ ), 6.33 (major, $1 \mathrm{H}$, dd, $J=9.8 \mathrm{~Hz}, 1.3 \mathrm{~Hz}, \mathrm{COC} \underline{\mathrm{H}}=\mathrm{CH}$ ), 6.62 (minor, $1 \mathrm{H}, \mathrm{d}, J=9.8 \mathrm{~Hz}$, $\mathrm{COCH}=\mathrm{CH}$ ), 6.70 (major, $1 \mathrm{H}, \mathrm{d}, J=9.8 \mathrm{~Hz}, \mathrm{COCH}=\mathrm{C} \underline{\mathrm{H}}$ ); For the major diasteroisomer: 
$\delta_{\mathrm{C}}\left(125 \mathrm{MHz}, \mathrm{CDCl}_{3}\right) 23.4\left(\underline{\mathrm{CH}}_{2} \mathrm{CH}_{2} \mathrm{C}_{8} \mathrm{~F}_{17}\right), 27.5\left(3 \times \underline{\mathrm{CH}}_{3} \mathrm{C}\right), 32.2\left(\mathrm{t}, J^{2}{ }_{\mathrm{CF}}=23 \mathrm{~Hz}\right.$,

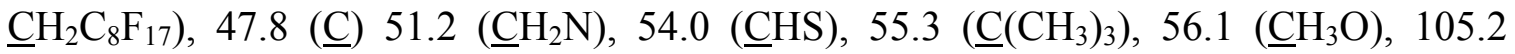
$(\underline{\mathrm{C}}), 129.7(\underline{\mathrm{C}}), 143.2(\underline{\mathrm{C}}), 169.7\left(\underline{\mathrm{C}}\left(\mathrm{OCH}_{3}\right)\right), 171.4(\mathrm{~N} \underline{\mathrm{CO}}), 186.4(\underline{\mathrm{CO}}) ; v_{\max }$ $\left(\mathrm{CH}_{2} \mathrm{Cl}_{2}\right.$ evaporated film/ $\left.\mathrm{cm}^{-1}\right) 3054$ and $2979(\mathrm{CH}), 1696(\mathrm{C}=\mathrm{O}), 1664$ (lactam $\left.\mathrm{C}=\mathrm{O}\right)$, 1632 and $1599(\mathrm{C}=\mathrm{C}) ; \mathrm{m} / \mathrm{z}\left(\mathrm{ES}^{+}\right) 745\left(\mathrm{M}+\mathrm{NH}_{4}{ }^{+}, 100 \%\right), 728\left(\mathrm{MH}^{+}, 31 \%\right), 276(29 \%)$, $174(42 \%) ; \mathrm{C}_{24} \mathrm{H}_{22} \mathrm{NO}_{3} \mathrm{~F}_{17} \mathrm{SNa}(\mathrm{M}+\mathrm{Na})$ found 750.0944 , expected 750.0941.

rac-(4R,5R)-4-(1H,1H,2H,2H-Heptadecafluorodecylsulfanyl)-2-iso-propyl-6methoxy-2-aza spiro-[4.5]-deca-6,9-diene-3,8-dione anti-19 rac-(4R,5S)-4-(1H,1H,2H,2H-Heptadecafluorodecylsulfanyl)-2-iso-propyl-6methoxy-2-aza spiro-[4.5]-deca-6,9-diene-3,8-dione syn-19
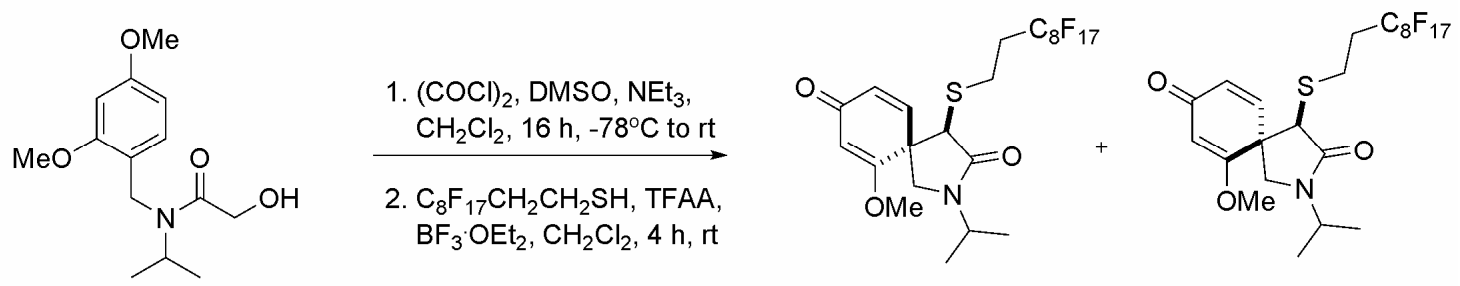

Following general procedure B: Treatment of the crude glyoxamide 6 (402 mg, 1.52 mmol, $1 \mathrm{eq})$ with $1 \mathrm{H}, 1 \mathrm{H}, 2 \mathrm{H}, 2 \mathrm{H}$-perfluorodecane-1-thiol $(0.31 \mathrm{ml}, 1.06 \mathrm{mmol}, 0.7 \mathrm{eq})$ TFAA (1.9 ml, $13.7 \mathrm{mmol}, 9 \mathrm{eq})$ and $\mathrm{BF}_{3} \cdot \mathrm{OEt}_{2}(0.94 \mathrm{ml}, 7.98 \mathrm{mmol}, 5 \mathrm{eq})$ gave a 2:1 mixture of diastereoisomers, as a yellow oil. Purification by flash column chromatography on silica gel (20\%-80\% ethyl acetate/iso-hexane) afforded spirocycle anti-19, as a yellow solid (238 $\mathrm{mg}, 0.33 \mathrm{mmol}, 31 \%$ over two steps).

$\delta_{\mathrm{H}}\left(300 \mathrm{MHz}, \mathrm{CDCl}_{3}\right) 1.16\left(6 \mathrm{H}, \mathrm{d}, J=6.9 \mathrm{~Hz}, 2 \times \mathrm{C}_{3} \mathrm{CH}\right), \quad 2.22-2.62(2 \mathrm{H}, \mathrm{m}$, $\left.\mathrm{C}_{2} \mathrm{C}_{8} \mathrm{~F}_{17}\right), 2.82-2.89\left(2 \mathrm{H}, \mathrm{m}, \mathrm{CH}_{2} \mathrm{CH}_{2} \mathrm{C}_{8} \mathrm{~F}_{17}\right), 3.15(1 \mathrm{H}, \mathrm{d}, J=9.7 \mathrm{~Hz}, \mathrm{C} \underline{\mathrm{HHN}}), 3.70(1 \mathrm{H}$, d, $J=9.7 \mathrm{~Hz}, \mathrm{CH} \underline{\mathrm{HN}}), 3.82\left(3 \mathrm{H}, \mathrm{s}, \mathrm{C}_{3} \mathrm{O}\right), 4.08(1 \mathrm{H}, \mathrm{s}, \mathrm{C} \underline{\mathrm{HS}}), 4.45$ (1H, septet, $J=6.9$ $\left.\mathrm{Hz}, \mathrm{C} \underline{\mathrm{H}}\left(\mathrm{CH}_{3}\right)_{2}\right), 5.76(1 \mathrm{H}, \mathrm{d}, J=1.5 \mathrm{~Hz}, \mathrm{C} \underline{\mathrm{HCOCH}}=\mathrm{CH}), 6.30(1 \mathrm{H}, \mathrm{dd}, J=10.0 \mathrm{~Hz}, 1.5$ $\mathrm{Hz}, \mathrm{CHCOC} \underline{\mathrm{H}}=\mathrm{CH}), 6.62(1 \mathrm{H}, \mathrm{d}, J=10.0 \mathrm{~Hz}, \mathrm{COCH}=\mathrm{C} \underline{\mathrm{H}}), \delta_{\mathrm{C}}\left(100 \mathrm{MHz}, \mathrm{CDCl}_{3}\right) 19.9$ $\left(2 \times \underline{\mathrm{CH}}_{3} \mathrm{CH}\right), 23.9\left(\underline{\mathrm{CH}}_{2} \mathrm{CH}_{2} \mathrm{C}_{8} \mathrm{~F}_{17}\right), 32.7\left(\mathrm{t}, J^{2}{ }_{\mathrm{CF}}=23 \mathrm{~Hz}, \underline{\mathrm{CH}}_{2} \mathrm{C}_{8} \mathrm{~F}_{17}\right), 44.1\left(\underline{\mathrm{CH}}\left(\mathrm{CH}_{3}\right)_{2}\right)$, $48.5\left(\underline{\mathrm{C}} \mathrm{H}_{2} \mathrm{~N}\right), 48.7(\underline{\mathrm{C}}), 53.9(\underline{\mathrm{CHS}}), 56.5\left(\underline{\mathrm{CH}}_{3} \mathrm{O}\right), 105.6(\underline{\mathrm{CHCO}}), 130.0(\mathrm{CO} \underline{\mathrm{CH}}=\mathrm{CH})$, 
$143.3(\mathrm{COCH}=\underline{\mathrm{CH}}), 169.3\left(\underline{\mathrm{C}}\left(\mathrm{OCH}_{3}\right)\right), 171.9(\mathrm{~N} \underline{\mathrm{CO}}), 186.7(\underline{\mathrm{CO}}) ; v_{\max }\left(\mathrm{CH}_{2} \mathrm{Cl}_{2}\right.$ evaporated film/ $\left.\mathrm{cm}^{-1}\right) 2978$ and $2947(\mathrm{CH}), 1696(\mathrm{C}=\mathrm{O}), 1664$ (lactam $\left.\mathrm{C}=\mathrm{O}\right), 1599$ $(\mathrm{C}=\mathrm{C}) ; \mathrm{m} / \mathrm{z}\left(\mathrm{ES}^{+}\right) 736(\mathrm{M}+\mathrm{Na}, 100 \%), 714\left(\mathrm{MH}^{+}, 8 \%\right), 542$ (21\%), 441 (30\%), 210 (9\%); $\mathrm{C}_{23} \mathrm{H}_{20} \mathrm{NO}_{3} \mathrm{~F}_{17} \mathrm{SNa}$ found 736.0778 , expected 736.0785; $\mathrm{mp}\left(\mathrm{CH}_{2} \mathrm{Cl}_{2}\right) 72.4-74.9{ }^{\circ} \mathrm{C}$.

Continued elution gave a 3:2 anti:syn mixture of 19 (139 mg, $0.19 \mathrm{mmol}, 18 \%$ over two steps), then afforded syn-19 as yellow oil (160 $\mathrm{mg}, 0.22 \mathrm{mmol}, 21 \%)$.

$\delta_{\mathrm{H}}\left(300 \mathrm{MHz}, \mathrm{CDCl}_{3}\right) 1.14\left(3 \mathrm{H}, \mathrm{d}, J=6.9 \mathrm{~Hz}, \mathrm{C}_{3} \mathrm{CH}\right), 1.16(3 \mathrm{H}, \mathrm{d}, J=6.9 \mathrm{~Hz}$, $\left.\mathrm{C}_{3} \mathrm{CH}\right), 2.24-2.65\left(2 \mathrm{H}, \mathrm{m}, \mathrm{C}_{2} \mathrm{C}_{8} \mathrm{~F}_{17}\right), 2.83-2.90\left(2 \mathrm{H}, \mathrm{m}, \mathrm{C}_{2} \mathrm{CH}_{2} \mathrm{C}_{8} \mathrm{~F}_{17}\right), 3.42(1 \mathrm{H}, \mathrm{d}, J$ $=10.4 \mathrm{~Hz}, \mathrm{C} \underline{H H N}), 3.50(1 \mathrm{H}, \mathrm{d}, J=10.4 \mathrm{~Hz}, \mathrm{CH} \underline{H N}), 3.65(1 \mathrm{H}, \mathrm{s}, \mathrm{C} \underline{\mathrm{HS}}), 3.70(3 \mathrm{H}, \mathrm{s}$, $\left.\mathrm{C}_{3} \mathrm{O}\right), 4.43\left(1 \mathrm{H}\right.$, septet, $\left.J=6.9 \mathrm{~Hz}, \mathrm{C} \underline{\mathrm{H}}\left(\mathrm{CH}_{3}\right)_{2}\right), 5.62(1 \mathrm{H}, \mathrm{d}, J=1.5 \mathrm{~Hz}$, $\mathrm{C} \underline{\mathrm{HCOCH}}=\mathrm{CH}), 6.31(1 \mathrm{H}, \mathrm{dd}, J=9.8,1.5 \mathrm{~Hz}, \mathrm{CHCOC} \underline{\mathrm{H}}=\mathrm{CH}), 6.64(1 \mathrm{H}, \mathrm{dd}, J=9.8 \mathrm{~Hz}$, $1.5 \mathrm{~Hz}, \mathrm{CHCOCH}=\mathrm{C} \underline{\mathrm{H}}) ; \delta_{\mathrm{C}}\left(100 \mathrm{MHz}, \mathrm{CDCl}_{3}\right) 18.8\left(\underline{\mathrm{CH}}_{3} \mathrm{CH}\right), 19.5\left(\underline{\mathrm{CH}}_{3} \mathrm{CH}\right), 24.3$ $\left(\underline{\mathrm{CH}}_{2} \mathrm{CH}_{2} \mathrm{C}_{8} \mathrm{~F}_{17}\right), 32.5\left(\mathrm{t}, J_{\mathrm{CF}}^{2}=22 \mathrm{~Hz}, \underline{\mathrm{C}} \mathrm{H}_{2} \mathrm{C}_{8} \mathrm{~F}_{17}\right), 43.8\left(\underline{\mathrm{CH}}\left(\mathrm{CH}_{3}\right)_{2}\right), 47.9\left(\underline{\mathrm{C}} \mathrm{H}_{2} \mathrm{~N}\right), 48.3$ () $), 55.5\left(\underline{\mathrm{CH}}_{3} \mathrm{O}\right), 56.1(\underline{\mathrm{CHS}}), 103.5(\underline{\mathrm{CHCOCH}}=\mathrm{CH}), 130.3(\mathrm{CHCOCH}=\mathrm{CH}), 144.6$ $(\mathrm{CHCOCH}=\underline{\mathrm{CH}}), 169.3\left(\underline{\mathrm{C}}\left(\mathrm{OCH}_{3}\right)\right), 174.1(\mathrm{~N} \underline{\mathrm{CO}}), 186.7(\underline{\mathrm{CO}}) ; v_{\max }\left(\mathrm{CH}_{2} \mathrm{Cl}_{2}\right.$ evaporated film/ $\left.\mathrm{cm}^{-1}\right) 2978$ and $2936(\mathrm{CH}), 1695(\mathrm{C}=\mathrm{O}), 1664(\mathrm{NC}=\mathrm{O}), 1598(\mathrm{C}=\mathrm{C}) ; \mathrm{m} / \mathrm{z}\left(\mathrm{ES}^{+}\right) 736$ $(\mathrm{M}+\mathrm{Na}, 100 \%), 441$ (34\%), 240 (32\%), $210(34 \%) ; \mathrm{C}_{23} \mathrm{H}_{20} \mathrm{NO}_{3} \mathrm{~F}_{17} \mathrm{SNa}(\mathrm{M}+\mathrm{Na})$ found 736.0779, expected 736.0785 .

rac-(4R,5R)-4-Ethylsulfanyl-2-iso-propyl-6-methoxy-2-azaspiro-[4.5]-deca-6,9-diene3,8-dione anti-20

rac-(4R,5S)-4-Ethylsulfanyl-2-iso-propyl-6-methoxy-2-azaspiro-[4.5]-deca-6,9-diene3,8-dione syn-20
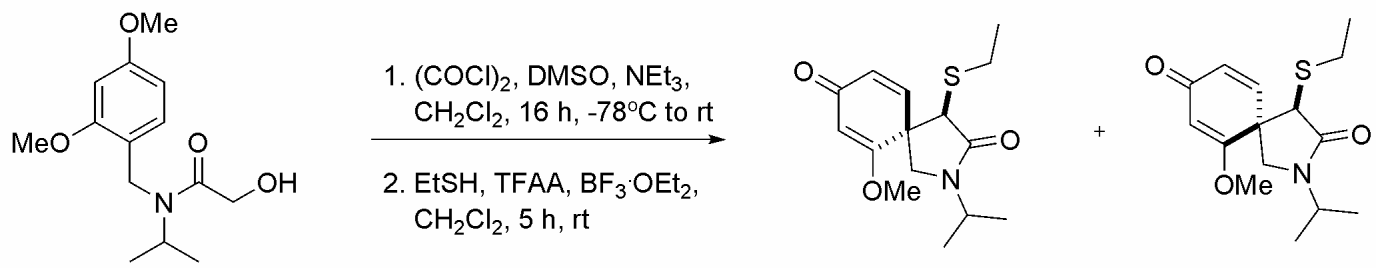

Following general procedure B: Treatment of the crude glyoxamide 6 (337 mg, 1.27 mmol, 1 eq) with ethanethiol $(0.09 \mathrm{ml}, 1.27 \mathrm{mmol}, 1 \mathrm{eq})$, TFAA (1.60 ml. $11.5 \mathrm{mmol}, 9$ 
eq) and $\mathrm{BF}_{3} \cdot \mathrm{OEt}_{2}(0.79 \mathrm{ml}, 6.36 \mathrm{mmol}, 5 \mathrm{eq})$ gave a yellow oil as a $3: 1$ mixture of diastereoisomers. Purification by flash column chromatography on silica gel (20\%-80\% ethyl acetate/iso-hexane) afforded spirocycle anti-20, as a yellow oil (140 mg, 0.47 mmol, $37 \%$ over 2 steps).

$\delta_{\mathrm{H}}\left(300 \mathrm{MHz}, \mathrm{CDCl}_{3}\right) 1.16\left(6 \mathrm{H}, \mathrm{d}, J=6.9 \mathrm{~Hz}, 2 \times \mathrm{C}_{3} \mathrm{CH}\right), 1.18(3 \mathrm{H}, \mathrm{t}, J=7.3 \mathrm{~Hz}$, $\left.\mathrm{C}_{3} \mathrm{CH}_{2}\right), 2.65\left(2 \mathrm{H}, \mathrm{qd}, J=7.3 \mathrm{~Hz}, 1.9 \mathrm{~Hz}, \mathrm{CH}_{3} \underline{\mathrm{C}}_{2}\right), 3.15(1 \mathrm{H}, \mathrm{d}, J=9.7 \mathrm{~Hz}, \mathrm{C} \underline{H} H \mathrm{H})$, $3.64(1 \mathrm{H}, \mathrm{d}, J=9.7 \mathrm{~Hz}, \mathrm{CH} \underline{\mathrm{HN}}), 3.81$ (3H, s, $\left.\underline{\mathrm{C}}_{3} \mathrm{O}\right), 4.09$ (1H, s, $\left.\mathrm{C} \underline{\mathrm{HS}}\right), 4.45$ (1H, septet, $\left.J=6.9 \mathrm{~Hz}, \mathrm{C} \underline{\mathrm{H}}\left(\mathrm{CH}_{3}\right)_{2}\right), 5.72(1 \mathrm{H}, \mathrm{d}, J=1.6 \mathrm{~Hz}, \mathrm{CHCOCH}=\mathrm{CH}), 6.28(1 \mathrm{H}, \mathrm{dd}, J=10.0$ $\mathrm{Hz}, 1.6 \mathrm{~Hz}, \mathrm{CHCOC} \underline{\mathrm{H}}=\mathrm{CH}), 6.67(1 \mathrm{H}, \mathrm{d}, J=10.0 \mathrm{~Hz}, \mathrm{CHCOCH}=\mathrm{CH}) ; \delta_{\mathrm{C}}(75 \mathrm{MHz}$, $\left.\mathrm{CDCl}_{3}\right) 14.6\left(\underline{\mathrm{CH}}_{3} \mathrm{CH}_{2} \mathrm{~S}\right), 19.8\left(2 \times \underline{\mathrm{CH}_{3}} \mathrm{CH}\right), 26.9\left(\mathrm{CH}_{3} \underline{\mathrm{C}} \mathrm{H}_{2} \mathrm{~S}\right), 43.8\left(\underline{\mathrm{CH}}\left(\mathrm{CH}_{3}\right)_{2}\right), 48.2$ $\left(\underline{\mathrm{C}} \mathrm{H}_{2} \mathrm{~N}\right), \quad 48.6 \quad(\underline{\mathrm{C}}), \quad 53.1 \quad(\underline{\mathrm{C}} \mathrm{HS}), \quad 56.4 \quad\left(\underline{\mathrm{C}}_{3} \mathrm{O}\right), \quad 105.0 \quad(\underline{\mathrm{C} H C O C H}=\mathrm{CH}), \quad 129.3$ $(\mathrm{CHCO} \underline{\mathrm{CH}}=\mathrm{CH}), 144.2(\mathrm{CHCOCH}=\underline{\mathrm{CH}}), 169.8\left(\underline{\mathrm{C}}\left(\mathrm{OCH}_{3}\right)\right), 172.6(\mathrm{NCO}), 187.0(\underline{\mathrm{CO}})$; $v_{\max }\left(\mathrm{CH}_{2} \mathrm{Cl}_{2}\right.$ evaporated film $\left./ \mathrm{cm}^{-1}\right) 2973$ and $2930(\mathrm{CH}), 1696(\mathrm{C}=\mathrm{O}), 1661$ (lactam $\mathrm{C}=\mathrm{O}), 1629$ and $1597(\mathrm{C}=\mathrm{C}) ; \mathrm{m} / \mathrm{z}\left(\mathrm{ES}^{+}\right) 296\left(\mathrm{MH}^{+}, 100 \%\right) ; \mathrm{C}_{15} \mathrm{H}_{22} \mathrm{NO}_{3} \mathrm{~S}\left(\mathrm{MH}^{+}\right)$found 296.1326, expected 296.1315.

Continued elution afforded spirocycle syn-20 as a yellow oil (86 mg, $0.29 \mathrm{mmol}, 23 \%$ over 2 steps).

$\delta_{\mathrm{H}}\left(300 \mathrm{MHz}, \mathrm{CDCl}_{3}\right) 1.14\left(3 \mathrm{H}, \mathrm{d}, J=6.7 \mathrm{~Hz}, \mathrm{C}_{3} \mathrm{CH}\right), 1.16(3 \mathrm{H}, \mathrm{d}, J=6.9 \mathrm{~Hz}$, $\left.\mathrm{C}_{3} \mathrm{CH}\right), 1.20\left(3 \mathrm{H}, \mathrm{t}, J=7.4 \mathrm{~Hz}, \mathrm{C}_{3} \mathrm{CH}_{2}\right), 2.61-2.74\left(2 \mathrm{H}, \mathrm{m}, \mathrm{CH}_{3} \underline{\mathrm{CH}}_{2}\right), 3.39(1 \mathrm{H}, \mathrm{d}, J=$ $10.4 \mathrm{~Hz}, \mathrm{C} \underline{H H N}), 3.48(1 \mathrm{H}, \mathrm{d}, J=10.4 \mathrm{~Hz}, \mathrm{CH} \underline{H N}), 3.69(1 \mathrm{H}, \mathrm{s}, \mathrm{C} \underline{H S}), 3.71(3 \mathrm{H}, \mathrm{s}$, $\left.\mathrm{C}_{3} \mathrm{O}\right), 4.44\left(1 \mathrm{H}\right.$, apparent septet, $\left.J=6.8 \mathrm{~Hz}, \mathrm{C} \underline{\mathrm{H}}\left(\mathrm{CH}_{3}\right)_{2}\right), 5.62(1 \mathrm{H}, \mathrm{d}, J=1.4 \mathrm{~Hz}$, $\mathrm{C} \underline{H C O C H}=\mathrm{CH}), 6.29(1 \mathrm{H}, \mathrm{dd}, J=9.8 \mathrm{~Hz}, 1.4 \mathrm{~Hz}, \mathrm{CHCOC} \underline{\mathrm{H}}=\mathrm{CH}), 6.64(1 \mathrm{H}, \mathrm{d}, J=9.8$ $\mathrm{Hz}, \mathrm{CHCOCH}=\mathrm{C} \underline{\mathrm{H}}) ; \delta_{\mathrm{C}}\left(75 \mathrm{MHz}, \mathrm{CDCl}_{3}\right) 15.0\left(\underline{\mathrm{CH}}_{3} \mathrm{CH}_{2} \mathrm{~S}\right), 19.2\left(\underline{\mathrm{C}}{ }_{3} \mathrm{CH}\right), 19.8$ $\left(\underline{\mathrm{CH}}_{3} \mathrm{CH}\right), 27.7\left(\mathrm{CH}_{3} \underline{\mathrm{CH}}_{2} \mathrm{~S}\right), 43.9\left(\underline{\mathrm{CH}}\left(\mathrm{CH}_{3}\right)_{2}\right), 48.0\left(\underline{\mathrm{CH}}_{2} \mathrm{~N}\right), 48.7(\underline{\mathrm{C}}), 55.5$ (ㅁS), 55.8 $\left(\underline{\mathrm{CH}}_{3} \mathrm{O}\right), 103.6(\underline{\mathrm{CHCOCH}}=\mathrm{CH}), 130.2(\mathrm{CHCO} \underline{\mathrm{CH}}=\mathrm{CH}), 145.6(\mathrm{CHCOCH}=\underline{\mathrm{CH}}), 170.0$ $\left(\underline{\mathrm{C}}\left(\mathrm{OCH}_{3}\right)\right), 175.3(\mathrm{~N} \underline{\mathrm{CO}}), 187.5(\underline{\mathrm{CO}}) ; v_{\max }\left(\mathrm{CH}_{2} \mathrm{Cl}_{2}\right.$ evaporated film/ $\left./ \mathrm{cm}^{-1}\right) 2973$ and $2941(\mathrm{CH}), 1693(\mathrm{C}=\mathrm{O}), 1660$ (lactam $\mathrm{C}=\mathrm{O}), 1626$ and $1595(\mathrm{C}=\mathrm{C}) ; \mathrm{m} / \mathrm{z}\left(\mathrm{ES}^{+}\right) 296$ $\left(\mathrm{MH}^{+}, 100 \%\right) ; \mathrm{C}_{15} \mathrm{H}_{22} \mathrm{NO}_{3} \mathrm{~S}\left(\mathrm{MH}^{+}\right)$found 296.1323, expected 296.1315 . 
rac-(4R,5R)-4-(1H,1H,2H,2H-Heptadecafluorodecylsulfanyl)-2-iso-propyl-6,9-

dimethoxy-2-aza spiro-[4.5]-deca-6,9-diene-3,8-dione anti-21

rac-(4R,5S)-4-(1H,1H,2H,2H-Heptadecafluorodecylsulfanyl)-2-iso-propyl-6,9-

dimethoxy-2-aza spiro-[4.5]-deca-6,9-diene-3,8-dione syn-21
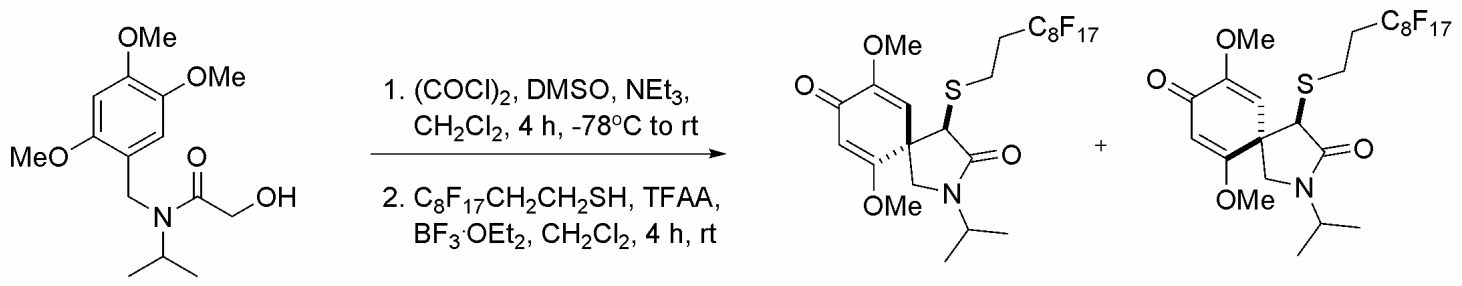

Following general procedure B: Treatment of the crude glyoxamide $7(0.45 \mathrm{~g}, 1.53$ mmol, 1 eq) with $1 \mathrm{H}, 1 \mathrm{H}, 2 \mathrm{H}, 2 \mathrm{H}-$ perfluorodecane-1-thiol $(0.31 \mathrm{ml}, 1.07 \mathrm{mmol}, 0.7 \mathrm{eq})$, TFAA (1.91 ml, $13.7 \mathrm{mmol}, 9 \mathrm{eq})$ and $\mathrm{BF}_{3} \cdot \mathrm{OEt}_{2}(0.95 \mathrm{ml}, 7.63 \mathrm{mmol}, 5 \mathrm{eq})$, then quench and work up with $1 \mathrm{M} \mathrm{NaOH}$ gave a 3:2 mixture of diastereoisomers, as a yellow oil. Purification by flash column chromatography on silica gel (50\%-80\% ethyl acetate/isohexane) afforded spirocycle anti-21, as a yellow oil (212 $\mathrm{mg}, 0.29 \mathrm{mmol}, 27 \%$ over two steps).

$\delta_{\mathrm{H}}\left(500 \mathrm{MHz}, \mathrm{CDCl}_{3}\right) 1.17\left(3 \mathrm{H}, \mathrm{d}, J=6.7 \mathrm{~Hz}, \mathrm{CH}_{3} \mathrm{CH}\right), 1.18(3 \mathrm{H}, \mathrm{d}, J=6.7 \mathrm{~Hz}$, $\left.\mathrm{C}_{3} \mathrm{CH}\right), 2.25-2.42\left(1 \mathrm{H}, \mathrm{m}, \mathrm{CH}_{2} \mathrm{CHHC}_{8} \mathrm{~F}_{17}\right), 2.45-2.60\left(1 \mathrm{H}, \mathrm{m}, \mathrm{CH}_{2} \mathrm{CH}_{\underline{H}} \mathrm{C}_{8} \mathrm{~F}_{17}\right), 2.82-$ $2.91\left(2 \mathrm{H}, \mathrm{m}, \underline{\mathrm{C}}_{2} \mathrm{CH}_{2} \mathrm{C}_{8} \mathrm{~F}_{17}\right), 3.17(1 \mathrm{H}, \mathrm{d}, J=9.6 \mathrm{~Hz}, \mathrm{C} \underline{\mathrm{HHN}}), 3.65\left(3 \mathrm{H}, \mathrm{s}, \mathrm{C}_{3} \mathrm{O}\right), 3.74$ $(1 \mathrm{H}, \mathrm{d}, J=9.6 \mathrm{~Hz}, \mathrm{CH} \underline{\mathrm{HN}}), 3.83\left(3 \mathrm{H}, \mathrm{s}, \mathrm{CH}_{3} \mathrm{O}\right), 4.03(1 \mathrm{H}, \mathrm{s}, \mathrm{C} \underline{\mathrm{HS}}), 4.47$ (1H, septet, $J=$ $\left.6.7 \mathrm{~Hz}, \mathrm{C} \underline{\mathrm{H}}\left(\mathrm{CH}_{3}\right)_{2}\right), 5.46(1 \mathrm{H}, \mathrm{s}, \mathrm{C} \underline{\mathrm{H}}), 5.81(1 \mathrm{H}, \mathrm{s}, \mathrm{C} \underline{\mathrm{H}}) ; \delta_{\mathrm{C}}\left(125 \mathrm{MHz}, \mathrm{CDCl}_{3}\right) 19.5(2 \times$

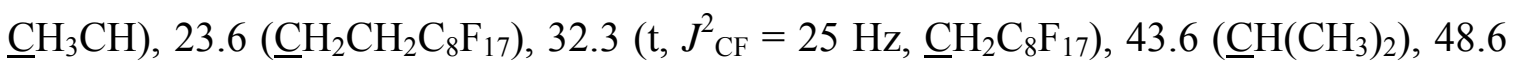
$(\underline{\mathrm{C}}), 49.4\left(\underline{\mathrm{CH}_{2}} \mathrm{~N}\right), 54.9(\underline{\mathrm{C}} \mathrm{HS}), 55.3\left(\underline{\mathrm{CH}}_{3} \mathrm{O}\right), 56.5\left(\underline{\mathrm{CH}}_{3} \mathrm{O}\right), 104.8(\underline{\mathrm{CH}}), 109.6(\underline{\mathrm{C}})$ ),

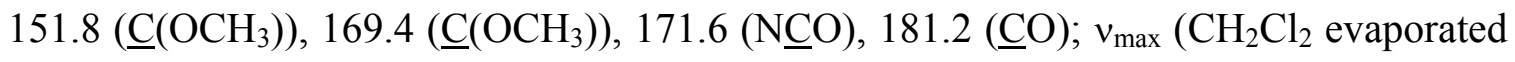
film/cm $\left.\mathrm{cm}^{-1}\right) 2977$ and $2941(\mathrm{CH}), 1696(\mathrm{C}=\mathrm{O}), 1664(\operatorname{lactam} \mathrm{C}=\mathrm{O}), 1610(\mathrm{C}=\mathrm{C}) ; \mathrm{m} / \mathrm{z}\left(\mathrm{ES}^{+}\right)$ $766(\mathrm{M}+\mathrm{Na}, 100 \%), 456(21 \%) ; \mathrm{C}_{24} \mathrm{H}_{22} \mathrm{NO}_{4} \mathrm{~F}_{17} \mathrm{SNa}(\mathrm{M}+\mathrm{Na})$ found 766.0877, expected 766.0890 .

Continued elution afforded a 1:1 anti:syn mixture of 21 (315 mg, $0.42 \mathrm{mmol}, 40 \%$ over two steps), then spirocycle syn-21 as yellow oil (36 mg, $0.048 \mathrm{mmol}, 5 \%$ over two steps) 
$\delta_{\mathrm{H}}\left(500 \mathrm{MHz}, \mathrm{CDCl}_{3}\right) 1.15\left(3 \mathrm{H}, \mathrm{d}, J=6.7 \mathrm{~Hz}, \mathrm{C}_{3} \mathrm{CH}\right), 1.17(3 \mathrm{H}, \mathrm{d}, J=6.7 \mathrm{~Hz}$, $\left.\mathrm{C}_{3} \mathrm{CH}\right), 2.31-2.45\left(1 \mathrm{H}, \mathrm{m}, \mathrm{CHHC}_{8} \mathrm{~F}_{17}\right), 2.46-2.62\left(1 \mathrm{H}, \mathrm{m}, \mathrm{CH}^{\mathrm{H} C} \mathrm{~F}_{8} \mathrm{~F}_{17}\right), 2.82-2.93(2 \mathrm{H}$, m, $\left.\underline{\mathrm{C}}_{2} \mathrm{CH}_{2} \mathrm{C}_{8} \mathrm{~F}_{17}\right), 3.44(1 \mathrm{H}, \mathrm{d}, J=10.2 \mathrm{~Hz}, \mathrm{C} \underline{\mathrm{HHN}}), 3.51(1 \mathrm{H}, \mathrm{d}, J=10.2 \mathrm{~Hz}, \mathrm{CH} \underline{\mathrm{HN}})$, $3.65(1 \mathrm{H}, \mathrm{s}, \mathrm{C} \underline{\mathrm{HS}}), 3.71\left(3 \mathrm{H}, \mathrm{s}, \mathrm{C}_{3} \mathrm{O}\right), 3.72\left(3 \mathrm{H}, \mathrm{s}, \mathrm{C}_{3} \mathrm{O}\right), 4.44(1 \mathrm{H}$, septet, $J=6.7 \mathrm{~Hz}$, $\left.\mathrm{C} \underline{\mathrm{H}}\left(\mathrm{CH}_{3}\right)_{2}\right), 5.45(1 \mathrm{H}, \mathrm{s}, \mathrm{C} \underline{\mathrm{H}}), 5.66(1 \mathrm{H}, \mathrm{s}, \mathrm{C} \underline{\mathrm{H}}) ; \delta_{\mathrm{C}}\left(100 \mathrm{MHz}, \mathrm{CDCl}_{3}\right) 18.8\left(\underline{\mathrm{C}} \mathrm{H}_{3} \mathrm{CH}\right)$, $19.5\left(\underline{\mathrm{C}} \mathrm{H}_{3} \mathrm{CH}\right), 24.4\left(\underline{\mathrm{CH}}_{2} \mathrm{CH}_{2} \mathrm{C}_{8} \mathrm{~F}_{17}\right), 32.7\left(\underline{\mathrm{CH}}_{2} \mathrm{C}_{8} \mathrm{~F}_{17}\right), 43.7\left(\underline{\mathrm{CH}}\left(\mathrm{CH}_{3}\right)_{2}\right), 48.2(\underline{\mathrm{C}}), 49.3$ $\left(\underline{\mathrm{CH}}_{2} \mathrm{~N}\right), 55.3\left(\underline{\mathrm{CH}}_{3} \mathrm{O}\right), 55.9\left(\underline{\mathrm{CH}}_{3} \mathrm{O}\right), 57.3(\underline{\mathrm{CHS}}), 103.0(\underline{\mathrm{CH}}), 111.5(\underline{\mathrm{CH}}), 151.9$ $\left(\underline{\mathrm{C}}\left(\mathrm{OCH}_{3}\right)\right), 169.6\left(\underline{\mathrm{C}}\left(\mathrm{OCH}_{3}\right)\right), 174.5(\mathrm{~N} \underline{\mathrm{CO}}), 181.4(\underline{\mathrm{CO}}) ; v_{\max }\left(\mathrm{CH}_{2} \mathrm{Cl}_{2}\right.$ evaporated film $\left./ \mathrm{cm}^{-1}\right) 2978$ and $2947(\mathrm{CH}), 1695(\mathrm{C}=\mathrm{O}), 1664$ (lactam $\left.\mathrm{C}=\mathrm{O}\right), 1603(\mathrm{C}=\mathrm{C}) ; \mathrm{m} / \mathrm{z}\left(\mathrm{ES}^{+}\right)$ $744\left(\mathrm{MH}^{+}, 100 \%\right), 214(19 \%) ; \mathrm{C}_{24} \mathrm{H}_{23} \mathrm{NO}_{4} \mathrm{~F}_{17} \mathrm{~S}\left(\mathrm{MH}^{+}\right)$found 744.1080, expected 744.1071 .

rac-(4R,5R)-4-Ethylsulfanyl-2-iso-propyl-6,9-dimethoxy-2-azaspiro-[4.5]-deca-6,9diene-3,8-dione anti-22 rac-(4R,5S)-4-ethylsulfanyl-2-iso-propyl-6,9-dimethoxy-2-azaspiro-[4.5]-deca-6,9diene-3,8-dione syn-22
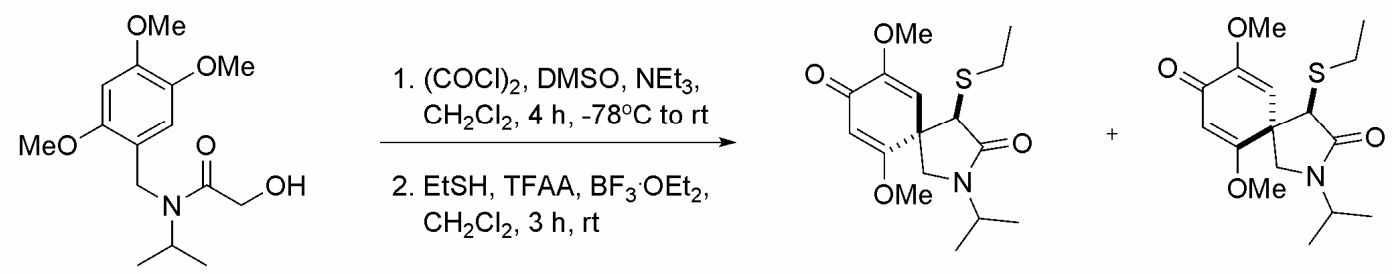

Following general procedure B: Treatment of the crude glyoxamide $7(0.24 \mathrm{~g}, 0.81$ mmol, 1 eq) with ethanethiol $(0.06 \mathrm{ml}, 0.81 \mathrm{mmol}, 1 \mathrm{eq})$, TFAA $(1.02 \mathrm{ml}, 7.32 \mathrm{mmol}, 9$ eq) and $\mathrm{BF}_{3} \cdot \mathrm{OEt}_{2}(0.51 \mathrm{ml}, 4.07 \mathrm{mmol}, 5 \mathrm{eq})$ gave a 3:1 mixture of diastereoisomers, as a yellow oil. Purification by flash column chromatography on silica gel (30\%-80\% ethyl acetate/iso-hexane) gave spirocycle anti-22 as a yellow amorphous solid (108 mg, 0.33 mmol, $41 \%$ over 2 steps).

$\delta_{\mathrm{H}}\left(400 \mathrm{MHz}, \mathrm{CDCl}_{3}\right) 1.17\left(3 \mathrm{H}, \mathrm{d}, J=6.8 \mathrm{~Hz}, \mathrm{C}_{3} \mathrm{CH}\right), 1.18(3 \mathrm{H}, \mathrm{d}, J=6.8 \mathrm{~Hz}$, $\left.\mathrm{C}_{3} \mathrm{CH}\right), 1.20\left(3 \mathrm{H}, \mathrm{t}, J=7.6 \mathrm{~Hz}, \mathrm{C}_{3} \mathrm{CH}_{2}\right), 2.62-2.76\left(2 \mathrm{H}, \mathrm{m}, \mathrm{CH}_{3} \underline{\mathrm{CH}}_{2}\right), 3.18(1 \mathrm{H}, \mathrm{d}, J=$ $9.6 \mathrm{~Hz}, \mathrm{C} \underline{\mathrm{HHN}}), 3.67\left(3 \mathrm{H}, \mathrm{s}, \mathrm{C}_{3} \mathrm{O}\right), 3.70(1 \mathrm{H}, \mathrm{d}, J=9.6 \mathrm{~Hz}, \mathrm{CH} \underline{\mathrm{HN}}), 3.83(3 \mathrm{H}, \mathrm{s}$, 
$\left.\mathrm{C}_{3} \mathrm{O}\right), 4.05(1 \mathrm{H}, \mathrm{s}, \mathrm{C} \underline{\mathrm{HS}}), 4.48\left(1 \mathrm{H}\right.$, septet, $\left.J=6.8 \mathrm{~Hz}, \mathrm{C} \underline{\mathrm{H}}\left(\mathrm{CH}_{3}\right)_{2}\right), 5.54(1 \mathrm{H}, \mathrm{s}, \mathrm{C} \underline{\mathrm{H}})$, $5.79(1 \mathrm{H}, \mathrm{s}, \mathrm{C} \underline{\mathrm{H}}) ; \delta_{\mathrm{C}}\left(100 \mathrm{MHz}, \mathrm{CDCl}_{3}\right) 14.5\left(\underline{\mathrm{CH}}_{3} \mathrm{CH}_{2}\right), 19.6\left(2 \times \underline{\mathrm{CH}}_{3} \mathrm{CH}\right), 26.7$ $\left(\mathrm{CH}_{3} \underline{\mathrm{CH}}_{2}\right), 43.4\left(\underline{\mathrm{C}} \mathrm{H}_{2} \mathrm{~N}\right), 48.7\left(\underline{\mathrm{CH}}\left(\mathrm{CH}_{3}\right)_{2}\right), 49.3(\underline{\mathrm{C}}), 54.0(\underline{\mathrm{C}} \mathrm{HS}), 55.4\left(\underline{\mathrm{CH}}_{3} \mathrm{O}\right), 56.4$ $\left(\underline{\mathrm{CH}}_{3} \mathrm{O}\right), 104.4(\underline{\mathrm{CH}}), 110.7(\underline{\mathrm{CH}}), 151.5\left(\underline{\mathrm{C}}\left(\mathrm{OCH}_{3}\right)\right), 170.0\left(\underline{\mathrm{C}}\left(\mathrm{OCH}_{3}\right)\right), 172.4(\mathrm{NCO})$, $181.6(\underline{\mathrm{CO}}) ; v_{\max }\left(\mathrm{CH}_{2} \mathrm{Cl}_{2}\right.$ evaporated film/ $\left.\mathrm{cm}^{-1}\right)$ 3071, 2983, 2936 and $2849(\mathrm{CH}), 1690$ $(\mathrm{C}=\mathrm{O}), 1650($ lactam $\mathrm{C}=\mathrm{O}), 1611(\mathrm{C}=\mathrm{C}) ; \mathrm{m} / \mathrm{z}\left(\mathrm{ES}^{+}\right) 348(\mathrm{M}+\mathrm{Na}, 11 \%), 326\left(\mathrm{MH}^{+}\right.$, $100 \%) ; \mathrm{C}_{16} \mathrm{H}_{24} \mathrm{NO}_{4} \mathrm{~S}\left(\mathrm{MH}^{+}\right)$found 326.1424, expected 326.1421 .

Continued elution gave spirocycle syn-22 as a yellow oil (35 mg, $0.11 \mathrm{mmol}, 13 \%$, over 2 steps).

$\delta_{\mathrm{H}}\left(400 \mathrm{MHz}, \mathrm{CDCl}_{3}\right) 1.14\left(3 \mathrm{H}, \mathrm{d}, J=6.8 \mathrm{~Hz}, \underline{\mathrm{CH}}_{3} \mathrm{CH}\right), 1.16(3 \mathrm{H}, \mathrm{d}, J=6.8 \mathrm{~Hz}$, $\left.\mathrm{C}_{3} \mathrm{CH}\right), 1.21\left(3 \mathrm{H}, \mathrm{t}, J=7.4 \mathrm{~Hz}, \mathrm{CH}_{3} \mathrm{CH}_{2}\right), 2.73-2.62\left(2 \mathrm{H}, \mathrm{m}, \mathrm{CH}_{3} \mathrm{CH}_{2}\right), 3.41(1 \mathrm{H}, \mathrm{d}, J=$

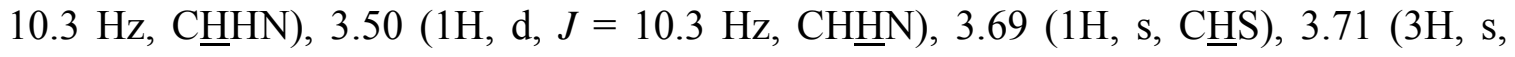
$\left.\mathrm{C}_{3} \mathrm{O}\right), 3.72\left(3 \mathrm{H}, \mathrm{s}, \underline{\mathrm{C}}_{3} \mathrm{O}\right), 4.45\left(1 \mathrm{H}\right.$, septet, $\left.J=6.8 \mathrm{~Hz}, \mathrm{C} \underline{\mathrm{H}}\left(\mathrm{CH}_{3}\right)_{2}\right), 5.45(1 \mathrm{H}, \mathrm{s}, \mathrm{C} \underline{\mathrm{H}})$, $5.65(1 \mathrm{H}, \mathrm{s}, \mathrm{C} \underline{\mathrm{H}}) ; \delta_{\mathrm{C}}\left(100 \mathrm{MHz}, \mathrm{CDCl}_{3}\right) 15.1\left(\underline{\mathrm{CH}}_{3} \mathrm{CH}_{2}\right), 19.3\left(\underline{\mathrm{CH}}_{3} \mathrm{CH}\right), 20.0\left(\underline{\mathrm{CH}}_{3} \mathrm{CH}\right)$, $27.9\left(\mathrm{CH}_{3} \underline{\mathrm{CH}_{2} \mathrm{~S}}\right), 43.9\left(\underline{\mathrm{CH}}\left(\mathrm{CH}_{3}\right)_{2}\right), 49.0\left(\underline{\mathrm{CH}_{2} \mathrm{~N}}\right), 49.5(\underline{\mathrm{C}}), 55.6\left(\underline{\left.\mathrm{CH}_{3} \mathrm{O}\right),}, 56.2\left(\underline{\mathrm{C}} \mathrm{H}_{3} \mathrm{O}\right)\right.$,

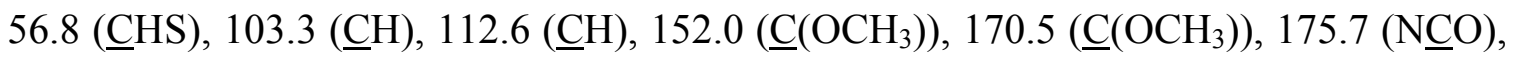
182.2 ( $\underline{\mathrm{CO}}) ; v_{\max }\left(\mathrm{CH}_{2} \mathrm{Cl}_{2}\right.$ evaporated film/ $\left./ \mathrm{cm}^{-1}\right) 2972$ and $2929(\mathrm{CH}), 1689(\mathrm{C}=\mathrm{O}), 1662$ (lactam $\mathrm{C}=\mathrm{O}), 1642$ and $1606(\mathrm{C}=\mathrm{C}) ; \mathrm{m} / \mathrm{z}\left(\mathrm{ES}^{+}\right) 348(\mathrm{M}+\mathrm{Na}, 12 \%), 326\left(\mathrm{MH}^{+}, 100 \%\right)$; $\mathrm{C}_{16} \mathrm{H}_{24} \mathrm{NO}_{4} \mathrm{~S}\left(\mathrm{MH}^{+}\right)$found 326.1424, expected 326.1421.

\section{rac-(4R,5R)-4-(1H,1H,2H,2H-Heptadecafluorodecylsulfanyl)-2-iso-propyl-6,7-} dimethoxy-2-aza spiro-[4.5]-deca-6,9-diene-3,8-dione 23
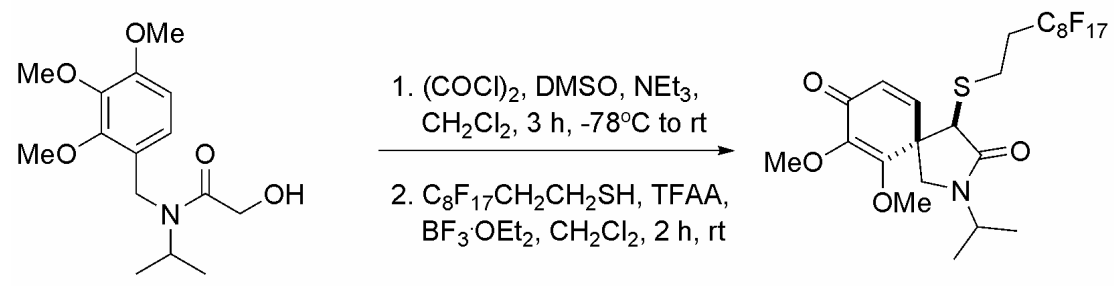

Treatment of the crude glyoxamide $8(720 \mathrm{mg}, 2.44 \mathrm{mmol}, 1 \mathrm{eq})$ with $1 \mathrm{H}, 1 \mathrm{H}, 2 \mathrm{H}, 2 \mathrm{H}-$ perfluorodecane-1-thiol (0.50 ml, $1.71 \mathrm{mmol}, 0.7 \mathrm{eq})$, TFAA (3.06 ml, $22.0 \mathrm{mmol}, 9 \mathrm{eq})$ and $\mathrm{BF}_{3} \cdot \mathrm{OEt}_{2}(1.51 \mathrm{ml}, 12.2 \mathrm{mmol}, 5 \mathrm{eq})$ gave a yellow solid. Purification by flash 
column chromatography on silica gel (20-70\% ethyl acetate/petroleum ether) afforded anti-spirocycle 23 as a yellow foam (690 mg, $0.93 \mathrm{mmol}, 54 \%$ over 2 steps).

$\delta_{\mathrm{H}}\left(500 \mathrm{MHz}, \mathrm{CDCl}_{3}\right) 1.15\left(3 \mathrm{H}, \mathrm{d}, J=6.0 \mathrm{~Hz}, \mathrm{CH}_{3} \mathrm{CH}\right), 1.17(3 \mathrm{H}, \mathrm{d}, J=6.0 \mathrm{~Hz}$, $\mathrm{C}_{3} \mathrm{CH}$ ), 2.30-2.60 (2H, m, $\left.\mathrm{CH}_{2} \mathrm{C}_{2} \mathrm{C}_{8} \mathrm{~F}_{17}\right), 2.83-2.94\left(2 \mathrm{H}, \mathrm{m}, \mathrm{C}_{2} \mathrm{CH}_{2} \mathrm{C}_{8} \mathrm{~F}_{17}\right), 3.10$ (1H, d, $J=9.7 \mathrm{~Hz}, \mathrm{C} \underline{H} H \mathrm{~N}), 3.68(1 \mathrm{H}, \mathrm{d}, J=9.7 \mathrm{~Hz}, \mathrm{CH} \underline{\mathrm{HN}}), 3.77\left(3 \mathrm{H}, \mathrm{s}, \mathrm{C}_{3} \mathrm{O}\right), 4.06(1 \mathrm{H}, \mathrm{s}$, C토), $4.18\left(3 \mathrm{H}, \mathrm{s}, \underline{\mathrm{C}}_{3} \mathrm{O}\right), 4.45\left(1 \mathrm{H}\right.$, septet, $\left.J=6.0 \mathrm{~Hz}, \mathrm{C} \underline{\mathrm{H}}\left(\mathrm{CH}_{3}\right)_{2}\right), 6.28(1 \mathrm{H}, \mathrm{d}, J=9.9$ $\mathrm{Hz}, \mathrm{C} \underline{H}=\mathrm{CH}), 5.54(1 \mathrm{H}, \mathrm{d}, J=9.9 \mathrm{~Hz}, \mathrm{CH}=\mathrm{C} \underline{\mathrm{H}}) ; \delta_{\mathrm{C}}\left(125 \mathrm{MHz}, \mathrm{CDCl}_{3}\right) 19.5(2 \times$ $\left.\underline{\mathrm{CH}_{3} \mathrm{CH}}\right), 23.7\left(\underline{\mathrm{CH}}_{2} \mathrm{CH}_{2} \mathrm{C}_{8} \mathrm{~F}_{17}\right), 32.2\left(\mathrm{t}, \mathrm{J}^{2}{ }_{\mathrm{CF}}=21 \mathrm{~Hz}, \underline{\mathrm{CH}}_{2} \mathrm{C}_{8} \mathrm{~F}_{17}\right), 43.6\left(\underline{\mathrm{CH}}\left(\mathrm{CH}_{3}\right)_{2}\right), 47.5$ $\left(\underline{\mathrm{CH}}_{2} \mathrm{~N}\right), 50.5(\underline{\mathrm{C}}), 53.3$ (때), $60.8\left(\underline{\mathrm{CH}}_{3} \mathrm{O}\right), 61.2\left(\underline{\mathrm{CH}}_{3} \mathrm{O}\right), 129.4(\underline{\mathrm{CH}}=\mathrm{CH}), 139.4$ $\left(\underline{\mathrm{C}}\left(\mathrm{OCH}_{3}\right)\right), 142.3(\mathrm{CH}=\underline{\mathrm{C}} \mathrm{H}), 157.3\left(\underline{\mathrm{C}}\left(\mathrm{OCH}_{3}\right)\right), 171.2(\mathrm{~N} \underline{\mathrm{CO}}), 183.4(\underline{\mathrm{CO}}) ; v_{\max }\left(\mathrm{CH}_{2} \mathrm{Cl}_{2}\right.$ evaporated film/cm $\left.{ }^{-1}\right) 1697(\mathrm{C}=\mathrm{O}) 1665$ (lactam $\left.\mathrm{C}=\mathrm{O}\right) 1604(\mathrm{C}=\mathrm{C}) ; \mathrm{m} / \mathrm{z}\left(\mathrm{ES}^{+}\right) 799$ $(\mathrm{M}+\mathrm{Na}, 100 \%), 744\left(\mathrm{MH}^{+}, 18 \%\right), 236(23 \%) ; \mathrm{C}_{24} \mathrm{H}_{22} \mathrm{NO}_{4} \mathrm{~F}_{17} \mathrm{SNa}(\mathrm{M}+\mathrm{Na})$ found 766.0892, expected 766.0890.

rac-(4R,5R)-4-(1H,1H,2H,2H-Heptadecafluorodecylsulfanyl)-2-iso-propyl-6methoxy-7-methyl-2-aza spiro-[4.5]-deca-6,9-diene-3,8-dione 24
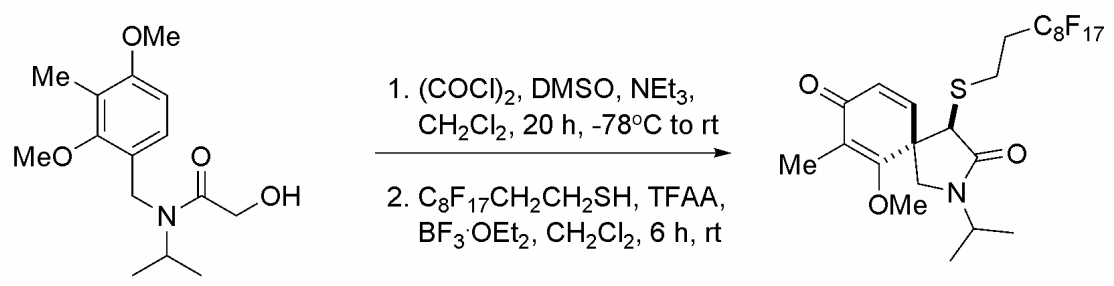

Treatment of the crude glyoxamide $9(430 \mathrm{mg}, 1.54 \mathrm{mmol}, 1 \mathrm{eq})$ with $1 \mathrm{H}, 1 \mathrm{H}, 2 \mathrm{H}, 2 \mathrm{H}-$ perfluorodecane-1-thiol (0.32 ml, $1.08 \mathrm{mmol}, 0.7 \mathrm{eq})$, TFAA (1.96 ml, $13.9 \mathrm{mmol}, 9 \mathrm{eq})$ and $\mathrm{BF}_{3} \cdot \mathrm{OEt}_{2}(0.97 \mathrm{ml}, 7.71 \mathrm{mmol}, 5 \mathrm{eq})$ gave an orange oil. Purification by flash column chromatography on silica gel (20\% ethyl acetate/petroleum ether) afforded antispirocycle 24 as a single diastereoisomer, as a yellow oil (394 mg, $0.53 \mathrm{mmol}, 50 \%$ over 2 steps).

$\delta_{\mathrm{H}}\left(500 \mathrm{MHz}, \mathrm{CDCl}_{3}\right) 1.18\left(6 \mathrm{H}, \mathrm{dd}, J=6.7 \mathrm{~Hz}, 2 \times \underline{\mathrm{CH}}_{3} \mathrm{CH}\right), 2.00\left(3 \mathrm{H}, \mathrm{s}, \mathrm{C}_{3} \mathrm{CCO}\right)$, 2.30-2.64 (2H, m, $\left.\mathrm{CH}_{2} \mathrm{C}_{2} \mathrm{C}_{8} \mathrm{~F}_{17}\right), 2.86-2.92\left(2 \mathrm{H}, \mathrm{m}, \mathrm{CH}_{2} \mathrm{CH}_{2} \mathrm{C}_{8} \mathrm{~F}_{17}\right), 3.09$ (1H, d, $J=9.5$ 


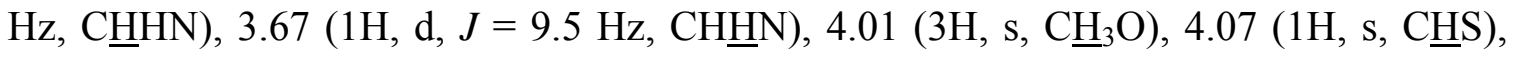
$4.47\left(1 \mathrm{H}\right.$, septet, $\left.J=6.7 \mathrm{~Hz}, \mathrm{C} \underline{\mathrm{H}}\left(\mathrm{CH}_{3}\right)_{2}\right), 6.36(1 \mathrm{H}, \mathrm{d}, J=10.1 \mathrm{~Hz}, \mathrm{C} \underline{H}=\mathrm{CH}), 5.63(1 \mathrm{H}, \mathrm{d}$, $J=10.1 \mathrm{~Hz}, \mathrm{CH}=\mathrm{C} \underline{\mathrm{H}}) ; \delta_{\mathrm{C}}\left(100 \mathrm{MHz}, \mathrm{CDCl}_{3}\right) 10.1\left(\underline{\mathrm{CH}_{3} \mathrm{CCO}}\right), 19.5\left(2 \times \underline{\mathrm{CH}_{3} \mathrm{CH}}\right), 23.8$ $\left(\underline{\mathrm{CH}_{2}} \mathrm{CH}_{2} \mathrm{C}_{8} \mathrm{~F}_{17}\right), 32.3\left(\mathrm{t}, J^{2}{ }_{\mathrm{CF}}=22 \mathrm{~Hz}, \underline{\mathrm{CH}}_{2} \mathrm{C}_{8} \mathrm{~F}_{17}\right), 43.6\left(\underline{\mathrm{CH}}\left(\mathrm{CH}_{3}\right)_{2}\right), 47.1\left(\underline{\mathrm{CH}_{2} \mathrm{~N}}\right), 49.9$

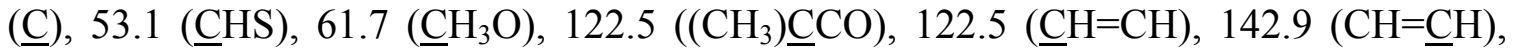
$167.2\left(\underline{\mathrm{C}}\left(\mathrm{OCH}_{3}\right)\right), 169.3(\mathrm{NCO}), 187.5(\underline{\mathrm{CO}}) ; v_{\max }\left(\mathrm{CH}_{2} \mathrm{Cl}_{2}\right.$ evaporated film/ $\left./ \mathrm{cm}^{-1}\right) 2977$ $(\mathrm{CH}), 1698(\mathrm{C}=\mathrm{O}) 1663(\operatorname{lactam} \mathrm{C}=\mathrm{O}), 1612(\mathrm{C}=\mathrm{C}) ; \mathrm{m} / \mathrm{z}\left(\mathrm{ES}^{+}\right) 745\left(\mathrm{M}+\mathrm{NH}_{4}^{+}, 100 \%\right)$, $728\left(\mathrm{MH}^{+}, 68 \%\right) ; \mathrm{C}_{24} \mathrm{H}_{26} \mathrm{~N}_{2} \mathrm{O}_{3} \mathrm{~F}_{17} \mathrm{~S}\left(\mathrm{M}+\mathrm{NH}_{4}{ }^{+}\right)$found 745.1389 , expected 745.1387.

\section{rac-2-(2-Fluoro-4-methoxyphenyl)- $N$-iso-propyl-2-(1H,1H,2H,2H- perfluorodecylsulfanyl)acetamide 26}
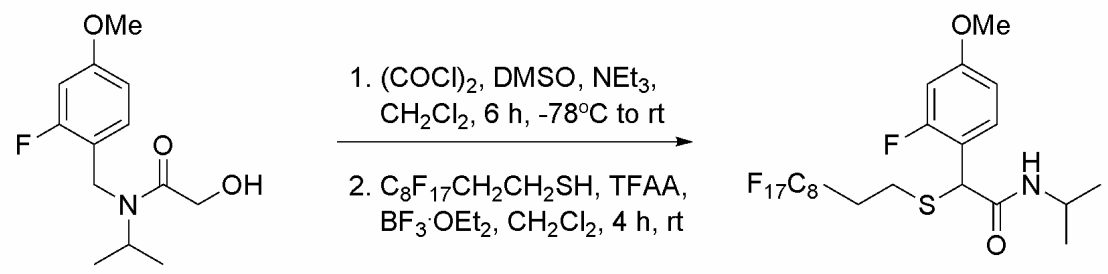

To oxalyl chloride $(0.22 \mathrm{ml}, 2.53 \mathrm{mmol}, 1.1 \mathrm{eq})$ in $\mathrm{CH}_{2} \mathrm{Cl}_{2}(10 \mathrm{ml})$ was added DMSO $(0.33 \mathrm{ml}, 4.60 \mathrm{mmol}, 2 \mathrm{eq})$ in $\mathrm{CH}_{2} \mathrm{Cl}_{2}(5 \mathrm{ml})$ via cannula at $-78{ }^{\circ} \mathrm{C}$. The mixture was then stirred for 30 minutes at $-78{ }^{\circ} \mathrm{C} . \quad N$-(2-Fluoro-4-methoxybenzyl)-2-hydroxy- $N$-iso-propyl acetamide $(0.59 \mathrm{~g}, 2.30 \mathrm{mmol}, 1 \mathrm{eq})$ in $\mathrm{CH}_{2} \mathrm{Cl}_{2}(10 \mathrm{ml})$ was added via cannula at $-78{ }^{\circ} \mathrm{C}$ and the mixture was stirred at this temperature for 1 hour. Triethylamine $(1.61 \mathrm{ml}, 11.5$ mmol, 5 eq) was added and the mixture allowed to warm to room temperature. The resulting yellow solution was stirred at room temperature for 6 hours. The organic layer was washed with aqueous saturated $\mathrm{NaHCO}_{3}(3 \times 25 \mathrm{ml})$, dried $\left(\mathrm{Na}_{2} \mathrm{SO}_{4}\right)$ and concentrated in vacuo to yield crude glyoxamide as a yellow foam. To the crude glyoxamide 25 (0.55 g, $2.17 \mathrm{mmol}, 1 \mathrm{eq})$ in $\mathrm{CH}_{2} \mathrm{Cl}_{2}(30 \mathrm{ml})$ was added $1 \mathrm{H}, 1 \mathrm{H}, 2 \mathrm{H}, 2 \mathrm{H}-$ perfluorodecane-1-thiol $(0.45 \mathrm{ml}, 1.52 \mathrm{mmol}, 0.7 \mathrm{eq})$ and the mixture was stirred at room temperature for 15 hours. Trifluoroacetic anhydride $(2.76 \mathrm{ml}, 19.6 \mathrm{mmol}, 9 \mathrm{eq})$ was added and the mixture was stirred at room temperature for 1 hour before the addition of $\mathrm{BF}_{3} \cdot \mathrm{OEt}_{2}(1.43 \mathrm{ml}, 10.9 \mathrm{mmol}, 5 \mathrm{eq})$. After 4 hours stirring at room temperature, during which time the solution became an intense purple colour, aqueous saturated $\mathrm{NaHCO}_{3}(20$ 
$\mathrm{ml}$ ) was added resulting in vigorous effervescence. The aqueous layer was extracted with $\mathrm{CH}_{2} \mathrm{Cl}_{2}(3 \times 30 \mathrm{ml})$ and the combined organic layers were washed with aqueous saturated $\mathrm{NaHCO}_{3}(3 \times 100 \mathrm{ml})$, dried $\left(\mathrm{Na}_{2} \mathrm{SO}_{4}\right)$ and concentrated in vacuo to afford a yellow oil. Purification by fluorous solid phase extraction on FluoroFlash ${ }^{\circledR}$ silica gel $(80 \%$ acetonitrile/water, then $100 \%$ acetonitrile) afforded the aryl migration product $\mathbf{2 6}$ as a yellow foam ( $0.65 \mathrm{~g}, 0.92 \mathrm{mmol}, 61 \%$ over 2 steps).

$\delta_{\mathrm{H}}\left(500 \mathrm{MHz}, \mathrm{CDCl}_{3}\right) 1.15\left(3 \mathrm{H}, \mathrm{d}, J=6.6 \mathrm{~Hz}, \mathrm{C}_{3} \mathrm{CH}\right), 1.18(3 \mathrm{H}, \mathrm{d}, J=6.6 \mathrm{~Hz}$, $\left.\mathrm{C}_{3} \mathrm{CH}\right), 2.28-2.45\left(2 \mathrm{H}, \mathrm{m}, \mathrm{CH}_{2} \mathrm{C}_{2} \mathrm{C}_{8} \mathrm{~F}_{17}\right), 2.72-2.82\left(2 \mathrm{H}, \mathrm{m}, \mathrm{C}_{2} \mathrm{CH}_{2} \mathrm{C}_{8} \mathrm{~F}_{17}\right), 3.79(3 \mathrm{H}$, s, $\left.\underline{\mathrm{C}}_{3} \mathrm{O}\right), 4.10\left(1 \mathrm{H}, \mathrm{m}, \mathrm{NHC} \underline{\mathrm{H}}\left(\mathrm{CH}_{3}\right)_{2}\right), 4.77(1 \mathrm{H}, \mathrm{s}, \mathrm{C} \underline{\mathrm{HS}}), 6.36(1 \mathrm{H}, \mathrm{d}, J=7.9 \mathrm{~Hz}$, $\mathrm{N} \underline{\mathrm{HCH}}), 6.63(1 \mathrm{H}, \mathrm{dd}, J=11.7 \mathrm{~Hz}, 2.5 \mathrm{~Hz}, \operatorname{Ar} \underline{\mathrm{H}}), 6.70(1 \mathrm{H}, \mathrm{dd}, J=8.5 \mathrm{~Hz}, 2.5 \mathrm{~Hz}, \operatorname{Ar} \underline{\mathrm{H}})$, $7.36(1 \mathrm{H}, \mathrm{t}, J=8.5 \mathrm{~Hz}, \mathrm{Ar} \underline{\mathrm{H}}) ; \delta_{\mathrm{C}}\left(125 \mathrm{MHz}, \mathrm{CDCl}_{3}\right) 22.5\left(2 \times\left(\underline{\mathrm{CH}}_{3}\right)_{2} \mathrm{CH}\right), 23.0$ $\left(\underline{\mathrm{CH}_{2}} \mathrm{CH}_{2} \mathrm{C}_{8} \mathrm{~F}_{17}\right), 31.5\left(\mathrm{t}, J_{\mathrm{CF}}^{2}=21 \mathrm{~Hz}, \mathrm{CH}_{2} \underline{\mathrm{CH}}_{2} \mathrm{C}_{8} \mathrm{~F}_{17}\right), 42.1\left(\underline{\mathrm{CH}}\left(\mathrm{CH}_{3}\right)_{2}\right), 47.0$ (ㄷS), $55.6\left(\underline{\mathrm{CH}}_{3} \mathrm{O}\right), 101.9\left(\mathrm{~d}, J_{\mathrm{CF}}^{2}=26 \mathrm{~Hz}, \mathrm{Ar} \underline{\mathrm{CH}}\right), 110.6\left(\mathrm{~d}, J_{\mathrm{CF}}^{4}=3 \mathrm{~Hz}, \mathrm{Ar} \underline{\mathrm{CH}}\right), 115.7\left(\mathrm{~d}, J_{\mathrm{CF}}^{3}\right.$ $=5 \mathrm{~Hz}, \operatorname{Ar} \underline{\mathrm{C}}), 129.8(\mathrm{Ar} \underline{\mathrm{C}} \mathrm{H}), 159.9\left(\mathrm{~d}, J_{\mathrm{CF}}^{3}=11 \mathrm{~Hz}, \operatorname{Ar} \underline{\mathrm{C}}\left(\mathrm{OCH}_{3}\right)\right), 161.8\left(\mathrm{~d}, J^{1}{ }_{\mathrm{CF}}=246\right.$ $\mathrm{Hz}, \mathrm{Ar} \underline{\mathrm{CF}}), 167.6$ (NCO); $v_{\max }\left(\mathrm{CH}_{2} \mathrm{Cl}_{2}\right.$ evaporated film/ $\left.\mathrm{cm}^{-1}\right) 3306$ (secondary $\mathrm{NH}$ ), 1651 (amide $\mathrm{C}=\mathrm{O}) ; \mathrm{m} / \mathrm{z}\left(\mathrm{ES}^{+}\right) 726(\mathrm{M}+23,100 \%), 704\left(\mathrm{MH}^{+}, 22 \%\right) ; \mathrm{C}_{22} \mathrm{H}_{20} \mathrm{NO}_{2} \mathrm{~F}_{18} \mathrm{~S}$ $\left(\mathrm{MH}^{+}\right)$found 704.0919, expected 704.0922.

rac-2-(2,6-Dimethoxyphenyl)- $N$-iso-propyl-2-(1H,1H,2H,2Hperfluorodecylsulfanyl)acetamide 28
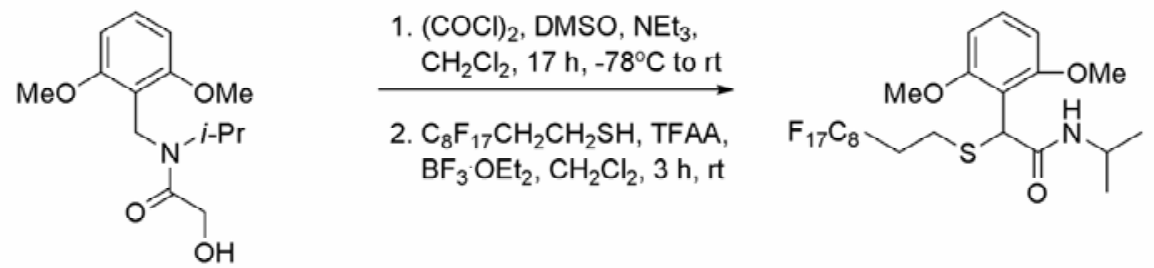

To oxalyl chloride $(0.08 \mathrm{ml}, 0.84 \mathrm{mmol}, 1.1 \mathrm{eq})$ in $\mathrm{CH}_{2} \mathrm{Cl}_{2}(5 \mathrm{ml})$ was added DMSO (0.13 ml, $1.87 \mathrm{mmol}, 2 \mathrm{eq})$ in $\mathrm{CH}_{2} \mathrm{Cl}_{2}(2 \mathrm{ml})$ via cannula at $-78{ }^{\circ} \mathrm{C}$. and the mixture was stirred for 1 hour at $-78{ }^{\circ} \mathrm{C}$. 2-Hydroxy- $N$-iso-propyl- $N$-(2,6-dimethoxybenzyl) acetamide (200 mg, $0.75 \mathrm{mmol}, 1 \mathrm{eq}$ ) in $\mathrm{CH}_{2} \mathrm{Cl}_{2}(5 \mathrm{ml})$ was added via cannula at $-78{ }^{\circ} \mathrm{C}$ and the mixture was stirred at this temperature for 1 hour. Triethylamine $(0.53 \mathrm{ml}, 3.74$ mmol, 5 eq) was added and the mixture allowed to warm to room temperature, then was 
stirred at room temperature for 17 hours. The organic layer was washed with aqueous saturated $\mathrm{NaHCO}_{3}(3 \times 20 \mathrm{ml})$, dried $\left(\mathrm{Na}_{2} \mathrm{SO}_{4}\right)$ and concentrated in vacuo to yield crude glyoxamide as a yellow oil. To the crude glyoxamide 27 (190 $\mathrm{mg}, 0.72 \mathrm{mmol}, 1 \mathrm{eq})$ in $\mathrm{CH}_{2} \mathrm{Cl}_{2}(8 \mathrm{ml})$ was added $1 \mathrm{H}, 1 \mathrm{H}, 2 \mathrm{H}, 2 \mathrm{H}$-perfluorodecane-1-thiol $(0.15 \mathrm{ml}, 0.50 \mathrm{mmol}$, 0.7 eq) and the mixture was stirred at room temperature for 18 hours. Trifluoroacetic anhydride $(0.91 \mathrm{ml}, 6.45 \mathrm{mmol}, 9 \mathrm{eq})$ was added and the mixture was stirred at room temperature for 1 hour before the addition of $\mathrm{BF}_{3} \cdot \mathrm{OEt}_{2}(0.45 \mathrm{ml}, 3.58 \mathrm{mmol}, 5 \mathrm{eq})$. After 3 hours stirring at room temperature, during which time the solution became an intense purple colour, aqueous saturated $\mathrm{NaHCO}_{3}(6 \mathrm{ml})$ was added resulting in vigorous effervescence. The aqueous layer was extracted with $\mathrm{CH}_{2} \mathrm{Cl}_{2}(3 \times 10 \mathrm{ml})$ and the combined organic layers were washed with aqueous saturated $\mathrm{NaHCO}_{3}(3 \times 30 \mathrm{ml})$, dried $\left(\mathrm{Na}_{2} \mathrm{SO}_{4}\right)$ and concentrated in vacuo to afford a yellow oil. Purification by flash column chromatography on silica gel (35\% ethyl acetate/petroleum ether) afforded aryl migration product 28 as a yellow solid (171 mg, $0.24 \mathrm{mmol}, 48 \%$ over 2 steps).

$\delta_{\mathrm{H}}\left(500 \mathrm{MHz}, \mathrm{CDCl}_{3}\right) 1.18\left(6 \mathrm{H}, \mathrm{d}, J=6.6 \mathrm{~Hz}, 2 \times \mathrm{C}_{3} \mathrm{CH}\right), 2.33-2.56(2 \mathrm{H}, \mathrm{m}$, $\left.\mathrm{CH}_{2} \underline{\mathrm{C}}_{2} \mathrm{C}_{8} \mathrm{~F}_{17}\right), 2.78\left(2 \mathrm{H}, \mathrm{t}, J=8.2 \mathrm{~Hz}, \underline{\mathrm{C}}_{2} \mathrm{CH}_{2} \mathrm{C}_{8} \mathrm{~F}_{17}\right), 3.82\left(6 \mathrm{H}, \mathrm{s}, 2 \times \mathrm{C}_{3} \mathrm{O}\right), 4.10$ $\left(1 \mathrm{H}, \mathrm{m}, \mathrm{NHC} \underline{\mathrm{H}}\left(\mathrm{CH}_{3}\right)_{2}\right), 5.05(1 \mathrm{H}, \mathrm{s}, \underline{\mathrm{CHS}}), 5.56(2 \mathrm{H}, \mathrm{d}, J=8.2 \mathrm{~Hz}, 2 \times \mathrm{Ar} \underline{\mathrm{H}}), 6.74(1 \mathrm{H}$, d, $J=7.9 \mathrm{~Hz}, \mathrm{~N} \underline{\mathrm{H}}), 7.23(1 \mathrm{H}, \mathrm{t}, J=8.2 \mathrm{~Hz}, \operatorname{Ar} \underline{\mathrm{H}}) ; \delta_{\mathrm{C}}\left(125 \mathrm{MHz}, \mathrm{CDCl}_{3}\right) 22.7(2 \times$ $\left.\underline{\mathrm{C}} \mathrm{H}_{3} \mathrm{CH}\right), 23.9\left(\underline{\mathrm{CH}}_{2} \mathrm{CH}_{2} \mathrm{C}_{8} \mathrm{~F}_{17}\right), 32.3\left(\mathrm{t}, J^{2}{ }_{\mathrm{CF}}=23 \mathrm{~Hz}, \mathrm{CH}_{2} \underline{\mathrm{CH}}_{2} \mathrm{C}_{8} \mathrm{~F}_{17}\right), 41.8\left(\underline{\mathrm{CH}}\left(\mathrm{CH}_{3}\right)_{2}\right)$, 44.7 (ㅁHS), $55.7\left(2 \times \underline{\mathrm{CH}}_{3} \mathrm{O}\right), 104.2(2 \times \mathrm{Ar} \underline{\mathrm{CH}}), 115.3(\mathrm{Ar} \underline{\mathrm{C}}), 129.4(\mathrm{Ar} \underline{\mathrm{CH}}), 157.6(2 \times$ $\left.\operatorname{Ar} \underline{C}\left(\mathrm{OCH}_{3}\right)\right), 168.8(\mathrm{~N} \underline{\mathrm{CO}}) ; v_{\max }\left(\mathrm{CH}_{2} \mathrm{Cl}_{2}\right.$ evaporated film/ $\left./ \mathrm{cm}^{-1}\right) 3369(\mathrm{NH}), 2970$ and $2936(\mathrm{CH}), 1665$ (amide $\mathrm{C}=\mathrm{O}), 1594$ and $1516(\mathrm{C}=\mathrm{C}) ; \mathrm{m} / \mathrm{z}\left(\mathrm{ES}^{+}\right) 738(100 \%, \mathrm{M}+\mathrm{Na})$, $426(42 \%) ; \mathrm{C}_{23} \mathrm{H}_{22} \mathrm{NO}_{3} \mathrm{SNa}(\mathrm{M}+\mathrm{Na})$ found 738.0942, expected 738.0941; mp $\left(\mathrm{CH}_{2} \mathrm{Cl}_{2}\right)$ $90.8-93.0^{\circ} \mathrm{C}$. 


\section{rac-4-Decylsulfanyl-2-iso-propyl-6,10-dimethoxy-2-azaspiro-[4.5]-deca-6,9-diene-}

\section{3,8-dione 35}
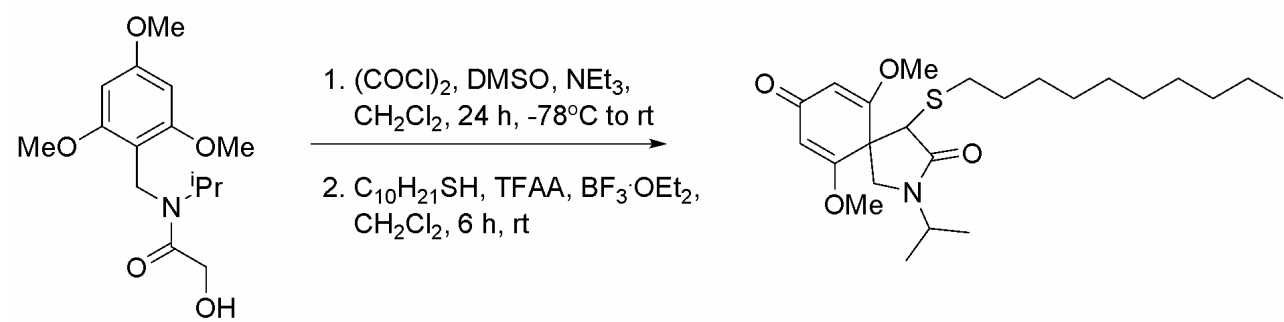

Following general procedure B: Treatment of the crude glyoxamide 2 (380 $\mathrm{mg}, 1.29$ mmol, 1 eq) with 1-decanethiol (0.27 ml, 1.29 mmol, 1 eq), TFAA (1.64 ml, $11.6 \mathrm{mmol}$, 9 eq) and $\mathrm{BF}_{3} \cdot \mathrm{OEt}_{2}(0.81 \mathrm{ml}, 6.44 \mathrm{mmol}, 5 \mathrm{eq})$ gave a yellow solid. Purification by flash column chromatography on silica gel (50\% ethyl acetate/petroleum ether) afforded spirocycle 35 as a yellow oil (280 mg, $0.64 \mathrm{mmol}, 50 \%$ over 2 steps).

$\delta_{\mathrm{H}}\left(500 \mathrm{MHz}, \mathrm{CDCl}_{3}\right) 0.88\left(3 \mathrm{H}, \mathrm{t}, J=6.9 \mathrm{~Hz}, \mathrm{CH}_{2} \mathrm{CH}_{3}\right), 1.13(3 \mathrm{H}, \mathrm{d}, J=6.9 \mathrm{~Hz}$, $\left.\mathrm{C}_{3} \mathrm{CH}\right), 1.16\left(3 \mathrm{H}, \mathrm{d}, J=6.9 \mathrm{~Hz}, \mathrm{C}_{3} \mathrm{CH}\right), 1.19-1.34\left(14 \mathrm{H}, \mathrm{m}, 7 \times \underline{\mathrm{C}}_{2}\right), 1.48(2 \mathrm{H}$, quintet, $\left.J=7.6 \mathrm{~Hz}, \mathrm{SCH}_{2} \underline{\mathrm{C}}_{2} \mathrm{CH}_{2}\right), 2.57\left(2 \mathrm{H}, \mathrm{t}, J=7.6 \mathrm{~Hz}, \mathrm{SC}_{2}\right), 3.37(1 \mathrm{H}, \mathrm{d}, J=9.5$ $\mathrm{Hz}, \mathrm{C} \underline{H} H \mathrm{~N}), 3.63(1 \mathrm{H}, \mathrm{d}, J=9.5 \mathrm{~Hz}, \mathrm{CH} \underline{\mathrm{HN}}), 3.68\left(3 \mathrm{H}, \mathrm{s}, \mathrm{CH}_{3} \mathrm{O}\right), 3.81\left(3 \mathrm{H}, \mathrm{s}, \mathrm{C}_{3} \mathrm{O}\right)$, $4.10(1 \mathrm{H}, \mathrm{s}, \mathrm{C} \underline{\mathrm{HS}}), 4.46\left(1 \mathrm{H}\right.$, septet, $\left.J=6.9 \mathrm{~Hz}, \underline{\mathrm{CH}}\left(\mathrm{CH}_{3}\right)_{2}\right), 5.53(1 \mathrm{H}, \mathrm{s}, \mathrm{C} \underline{\mathrm{HCOCH}}), 5.62$ $(1 \mathrm{H}, \mathrm{s}, \mathrm{CHCOC} \underline{\mathrm{H}}) ; \delta_{\mathrm{C}}\left(100 \mathrm{MHz}, \mathrm{CDCl}_{3}\right) 14.0\left(\underline{\mathrm{CH}}_{3} \mathrm{CH}_{2} \mathrm{CH}_{2}\right), 18.8\left(\underline{\mathrm{CH}}{ }_{3} \mathrm{CH}\right), 19.5$ $\left(\underline{\mathrm{CH}}_{3} \mathrm{CH}\right), 22.6\left(\underline{\mathrm{CH}}_{2}\right), 28.6\left(\underline{\mathrm{CH}}_{2}\right), 29.2\left(2 \times \underline{\mathrm{CH}}_{2}\right), 29.4\left(\underline{\mathrm{CH}}_{2}\right), 29.5\left(\underline{\mathrm{CH}}_{2}\right), 29.8\left(\underline{\mathrm{CH}}_{2}\right)$, $31.8\left(\underline{\mathrm{CH}}_{2}\right), 33.4\left(\underline{\mathrm{CH}}_{2} \mathrm{~S}\right), 43.6\left(\underline{\mathrm{C}} \mathrm{H}\left(\mathrm{CH}_{3}\right)_{2}\right), 47.4\left(\underline{\mathrm{CH}}_{2} \mathrm{~N}\right), 50.6(\underline{\mathrm{C}}), 54.4$ (ㅁHS), 55.7

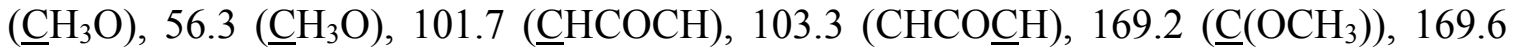
$\left(\underline{\mathrm{C}}\left(\mathrm{OCH}_{3}\right)\right), 172.8(\mathrm{NC} O), 187.1$ (ㅁ) $; v_{\max }\left(\right.$ neat $\left./ \mathrm{cm}^{-1}\right) 2962$ and $2854(\mathrm{CH}), 1693$ $(\mathrm{C}=\mathrm{O}), 1661$ (lactam $\mathrm{C}=\mathrm{O}), 1632$ and $1598(\mathrm{C}=\mathrm{C}) ; \mathrm{m} / \mathrm{z}\left(\mathrm{ES}^{+}\right) 438\left(\mathrm{MH}^{+}, 100 \%\right)$; $\mathrm{C}_{24} \mathrm{H}_{40} \mathrm{NO}_{4} \mathrm{~S}\left(\mathrm{MH}^{+}\right)$found 438.2678, expected 438.2673. 
rac-4-(1H,1H,2H,2H-Heptadecafluorodecylsulfinyl)-2-iso-propyl-6,10-dimethoxy-2aza-spiro-[4.5]-deca,6,9-diene-3,8-dione 29

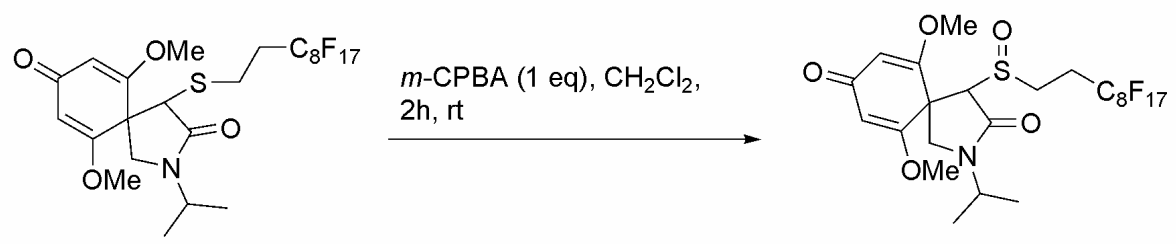

m-CPBA (244 mg, $1.08 \mathrm{mmol}, 1 \mathrm{eq})$ was added to sulfide 11 (800 mg, $1.08 \mathrm{mmol}, 1 \mathrm{eq})$ in $\mathrm{CH}_{2} \mathrm{Cl}_{2}(30 \mathrm{ml})$. The reaction mixture was stirred at room temperature for 2 hours then washed with aqueous saturated $\mathrm{NaHCO}_{3}$ solution $(3 \times 25 \mathrm{ml})$. The organic layer was dried $\left(\mathrm{MgSO}_{4}\right)$ and concentrated in vacuo to give spirocyclic sulfoxide 29 as a 1:1 mixture of diastereomers, as a yellow foam (759 mg, $1.00 \mathrm{mmol}, 93 \%)$. No further purification was required.

$\delta_{\mathrm{H}}\left(500 \mathrm{MHz}, \mathrm{CDCl}_{3}\right) 1.17$ (one diastereoisomer, $3 \mathrm{H}, \mathrm{d}, J=6.9 \mathrm{~Hz}, \mathrm{C}_{3} \mathrm{CH}$ ), 1.18 (one diastereoisomer, $3 \mathrm{H}, \mathrm{d}, J=6.9 \mathrm{~Hz}, \mathrm{CH}_{3} \mathrm{CH}$ ), 1.19 (one diastereoisomer, $3 \mathrm{H}, \mathrm{d}, J=6.9$ $\mathrm{Hz}, \underline{\mathrm{C}}_{3} \mathrm{CH}$ ), 1.22 (one diastereoisomer, $3 \mathrm{H}, \mathrm{d}, J=6.9 \mathrm{~Hz}, \underline{\mathrm{C}}_{3} \mathrm{CH}$ ), 2.43-2.72 (both diastereoisomers, $4 \mathrm{H}, \mathrm{m}, 2 \times \mathrm{CH}_{2} \mathrm{C}_{8} \mathrm{~F}_{17}$ ), 2.79 (one diastereoisomer, $1 \mathrm{H}$, ddd, $J=12.6$

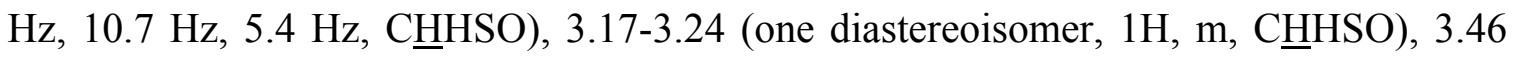
(one diastereoisomer, $1 \mathrm{H}, \mathrm{d}, J=9.8 \mathrm{~Hz}, \mathrm{C} \underline{H} \mathrm{HN}$ ), 3.59 (one diastereoisomer, $1 \mathrm{H}, \mathrm{d}, J=$

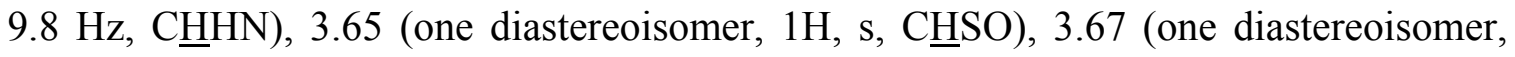
$1 \mathrm{H}, \mathrm{d}, J=9.8 \mathrm{~Hz}, \mathrm{CH} \underline{\mathrm{HN}}$ ), 3.72 (one diastereoisomer, $1 \mathrm{H}, \mathrm{d}, J=9.8 \mathrm{~Hz}, \mathrm{CH} \underline{H N}$ ), 3.78 (one diastereoisomer, $3 \mathrm{H}, \mathrm{s}, \mathrm{CH}_{3} \mathrm{O}$ ), 3.80 (both diastereoisomers, $6 \mathrm{H}, \mathrm{s}, 2 \times \mathrm{C}_{3} \mathrm{O}$ ), 3.84 (one diastereoisomer, $3 \mathrm{H}, \mathrm{s}, \mathrm{C}_{3} \mathrm{O}$ ), 3.82-3.89 (one diastereoisomer, 1H, s, CH$\underline{\mathrm{HSO}}$ ), 4.28-4.39 (one diastereoisomer, 1H, m, CHㅡSO and one diastereoisomer, $1 \mathrm{H}, \mathrm{m}$, $\left.\mathrm{C} \underline{\mathrm{H}}\left(\mathrm{CH}_{3}\right)_{2}\right), 4.41$ (one diastereoisomer, $1 \mathrm{H}, \mathrm{s}, \mathrm{C} \underline{\mathrm{HSO}}$ ), 4.52 (one diastereoisomer, $1 \mathrm{H}$ septet, $J=6.9 \mathrm{~Hz}, \mathrm{C} \underline{\mathrm{H}}\left(\mathrm{CH}_{3}\right)_{2}$ ), 5.59 (one diastereoisomer, 1H, s, $\mathrm{C} \underline{\mathrm{H}}$ ), 5.63 (one diastereoisomer, $1 \mathrm{H}, \mathrm{s}, \mathrm{C} \underline{\mathrm{H}}$ ), 5.66 (one diastereoisomer, $1 \mathrm{H}, \mathrm{s}, \mathrm{C} \underline{\mathrm{H}}$ ), 5.68 (one diastereoisomer, $1 \mathrm{H}, \mathrm{s}, \mathrm{C} \underline{\mathrm{H}}) ; \delta_{\mathrm{C}}\left(125 \mathrm{MHz}, \mathrm{CDCl}_{3}\right)$ one diastereomer $19.0\left(\underline{\mathrm{CH}}_{3} \mathrm{CH}\right), 19.3$ $\left(\underline{\mathrm{C}} \mathrm{H}_{3} \mathrm{CH}\right), 41.3\left(\underline{\mathrm{CH}}_{2}\right), 44.0\left(\underline{\mathrm{CH}}\left(\mathrm{CH}_{3}\right)_{2}\right), 47.5\left(\underline{\mathrm{CH}}_{2}\right), 48.0(\underline{\mathrm{C}}), 56.1\left(\underline{\mathrm{CH}}_{3} \mathrm{O}\right), 56.7$

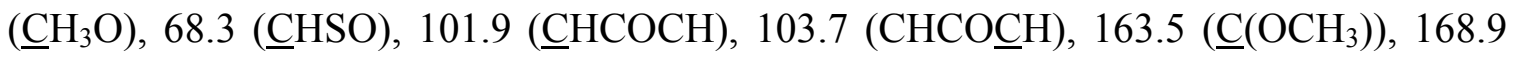


$\left(\underline{\mathrm{C}}\left(\mathrm{OCH}_{3}\right)\right), 169.9(\mathrm{NC} \mathrm{O}), 186.3(\underline{\mathrm{CO}}) ; v_{\max }\left(\right.$ neat $\left./ \mathrm{cm}^{-1}\right) 2979$ and $2941(\mathrm{CH}), 1688$ $(\mathrm{C}=\mathrm{O}), 1657$ (lactam $\mathrm{C}=\mathrm{O}), 1599(\mathrm{C}=\mathrm{C}) ; \mathrm{m} / \mathrm{z}\left(\mathrm{ES}^{+}\right) 782(\mathrm{M}+\mathrm{Na}, 22 \%), 760(100 \%$, $\left.\mathrm{MH}^{+}\right), 461(22 \%) ; \mathrm{C}_{24} \mathrm{H}_{23} \mathrm{NO}_{6} \mathrm{~F}_{17} \mathrm{~S}\left(\mathrm{MH}^{+}\right)$found 760.1022, expected 760.1020 .

rac-4-(1H,1H,2H,2H-Heptadecafluorodecylsulfonyl)-2-iso-propyl-6,10-dimethoxy-2aza-spiro-[4.5]-deca,6,9-diene-3,8-dione 30

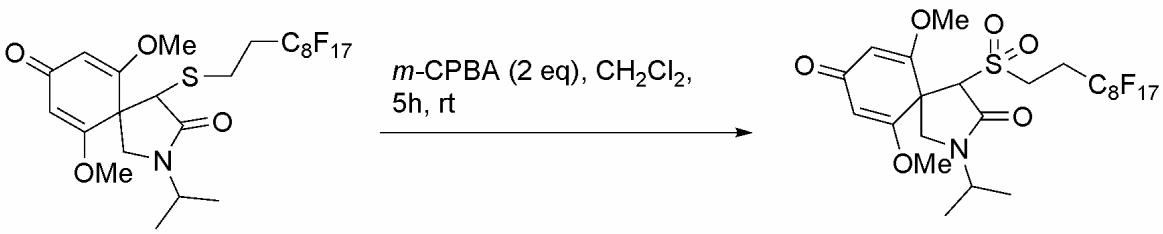

m-CPBA (304 mg, $1.35 \mathrm{mmol}, 2$ eq) was added to sulfide 11 (500 mg, $0.67 \mathrm{mmol}, 1 \mathrm{eq})$ in $\mathrm{CH}_{2} \mathrm{Cl}_{2}(20 \mathrm{ml})$. The reaction mixture was stirred at room temperature for 5 hours then washed with aqueous saturated $\mathrm{NaHCO}_{3}$ solution $(3 \times 20 \mathrm{ml})$ was added. The organic layer was dried $\left(\mathrm{MgSO}_{4}\right)$ and concentrated in vacuo to afford spirocyclic sulfone $\mathbf{3 0}$ as a yellow foam (400 mg, $0.52 \mathrm{mmol}, 78 \%$ ). No further purification was required.

$\delta_{\mathrm{H}}(500 \mathrm{MHz}, \mathrm{CDCl} 3) 1.19\left(3 \mathrm{H}, \mathrm{d}, J=6.9 \mathrm{~Hz}, \mathrm{CH}_{3} \mathrm{CH}\right), 1.20(3 \mathrm{H}, \mathrm{d}, J=6.6 \mathrm{~Hz}$, $\left.\mathrm{C}_{3} \mathrm{CH}\right), 2.55-2.79$ (2H, m, $\left.\mathrm{CH}_{2} \mathrm{CH}_{2} \mathrm{C}_{8} \mathrm{~F}_{17}\right), 3.42-3.51$ (1H, m, $\left.\mathrm{SO}_{2} \mathrm{C} \underline{\mathrm{HH}}\right), 3.47$ (1H, d, J $=9.6 \mathrm{~Hz}, \mathrm{C} \underline{\mathrm{HHN}}), 3.67(1 \mathrm{H}, \mathrm{d}, J=9.6 \mathrm{~Hz}, \mathrm{CH} \underline{\mathrm{HN}}), 3.73\left(3 \mathrm{H}, \mathrm{s}, \mathrm{C}_{3} \mathrm{O}\right), 3.85(3 \mathrm{H}, \mathrm{s}$, $\left.\mathrm{C}_{3} \mathrm{O}\right), 3.96\left(1 \mathrm{H}, \mathrm{ddd}, J=12.9 \mathrm{~Hz}, 12.6 \mathrm{~Hz}, 4.7 \mathrm{~Hz}, \mathrm{SO}_{2} \mathrm{CH} \underline{\mathrm{H}}\right), 4.44$ (1H, apparent septet, $\left.J=6.7 \mathrm{~Hz}, \mathrm{C} \underline{\mathrm{H}}\left(\mathrm{CH}_{3}\right)_{2}\right), 4.63\left(1 \mathrm{H}, \mathrm{s}, \mathrm{C}_{\mathrm{HSO}}\right), 5.61(1 \mathrm{H}, \mathrm{s}, \mathrm{C} \underline{\mathrm{HCOCH}}), 5.69(1 \mathrm{H}, \mathrm{s}$, $\mathrm{CHCOC} \underline{\mathrm{H}}) ; \delta_{\mathrm{C}}\left(125 \mathrm{MHz}, \mathrm{CDCl}_{3}\right) 19.0\left(\underline{\mathrm{CH}}_{3} \mathrm{CH}\right), 19.5\left(\underline{\mathrm{CH}}_{3} \mathrm{CH}\right), 23.3\left(\mathrm{t}, J_{\mathrm{CF}}^{2}=24 \mathrm{~Hz}\right.$,

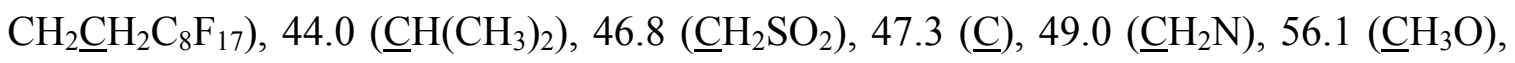
$56.8\left(\underline{\mathrm{CH}}_{3} \mathrm{O}\right), 68.5(\underline{\mathrm{CHSO}})_{2}, 102.2(\underline{\mathrm{CHCOCH}}), 103.7(\mathrm{CHCOC} H), 162.9\left(\underline{\mathrm{C}}\left(\mathrm{OCH}_{3}\right)\right)$,

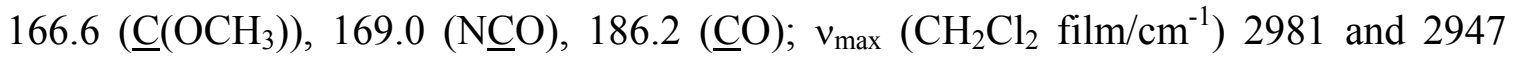
(CH), $1698(\mathrm{C}=\mathrm{O}), 1665$ (lactam $\mathrm{C}=\mathrm{O}), 1600(\mathrm{C}=\mathrm{C}) ; \mathrm{m} / \mathrm{z}\left(\mathrm{ES}^{+}\right) 798(\mathrm{M}+\mathrm{Na}, 70 \%), 576$ $(10 \%) ; \mathrm{C}_{24} \mathrm{H}_{22} \mathrm{NO}_{6} \mathrm{~F}_{17} \mathrm{Sna}(\mathrm{M}+\mathrm{Na})$ found 798.0809, expected 798.0789. 
rac-4-Ethylsulfonyl-2-iso-propyl-6,10-dimethoxy-2-aza-spiro-[4.5]-deca,6,9-diene3,8-dione 31
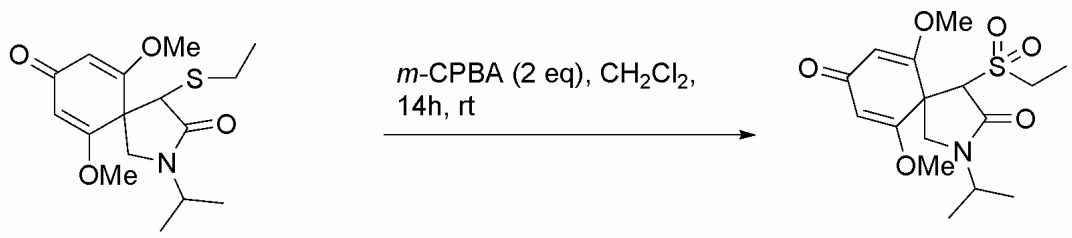

m-CPBA (73 mg, $0.35 \mathrm{mmol}, 2 \mathrm{eq})$ was added to sulfide 12 (56 mg, $0.17 \mathrm{mmol}, 1 \mathrm{eq})$ in $\mathrm{CH}_{2} \mathrm{Cl}_{2}(5 \mathrm{ml})$. The reaction mixture was stirred at room temperature for 14 hours then washed with aqueous saturated $\mathrm{NaHCO}_{3}$ solution $(3 \times 5 \mathrm{ml})$. The organic layer was $\left(\mathrm{Na}_{2} \mathrm{SO}_{4}\right)$ and concentrated in vacuo to give spirocyclic sulfone $\mathbf{3 1}$ as a yellow solid (57 $\mathrm{mg}, 0.16 \mathrm{mmol}, 92 \%)$. No further purification was required.

$\delta_{\mathrm{H}}(500 \mathrm{MHz}, \mathrm{CDCl}) 1.19\left(3 \mathrm{H}, \mathrm{d}, J=6.6 \mathrm{~Hz}, \mathrm{CH}_{3} \mathrm{CH}\right), 1.20(3 \mathrm{H}, \mathrm{d}, J=6.6 \mathrm{~Hz}$, $\left.\mathrm{C}_{3} \mathrm{CH}\right), 1.38\left(3 \mathrm{H}\right.$, apparent t, $\left.J=7.6 \mathrm{~Hz}, \underline{\mathrm{CH}}_{3} \mathrm{CH}_{2}\right), 3.39(1 \mathrm{H}, \mathrm{dd}, J=13.9 \mathrm{~Hz}, 7.3 \mathrm{~Hz}$, $\left.\mathrm{C}_{\text {HHSO }}\right), 3.45(1 \mathrm{H}, \mathrm{d}, J=9.6 \mathrm{~Hz}, \underline{\mathrm{CH}} \mathrm{HN}), 3.51(1 \mathrm{H}, \mathrm{dd}, J=13.9 \mathrm{~Hz}, J=8.6 \mathrm{~Hz}$ $\left.\mathrm{CH}_{\underline{H}} \mathrm{SO}_{2}\right), 3.65(1 \mathrm{H}, \mathrm{d}, J=9.6 \mathrm{~Hz}, \mathrm{CH} \underline{\mathrm{HN}}), 3.74\left(3 \mathrm{H}, \mathrm{s}, \mathrm{C}_{3} \mathrm{O}\right), 3.84\left(3 \mathrm{H}, \mathrm{s}, \mathrm{C}_{3} \mathrm{O}\right)$, $4.44\left(1 \mathrm{H}\right.$, septet, $\left.J=6.6 \mathrm{~Hz}, \mathrm{C} \underline{\mathrm{H}}\left(\mathrm{CH}_{3}\right)_{2}\right), 4.57\left(1 \mathrm{H}, \mathrm{s}, \mathrm{C}_{\mathbf{H S O}}\right), 5.60(1 \mathrm{H}, \mathrm{s}, \mathrm{C} \underline{\mathrm{HCOCH}})$, $5.67(1 \mathrm{H}, \mathrm{s}, \mathrm{CHCOC} \underline{\mathrm{H}}) ; \delta_{\mathrm{C}}\left(125 \mathrm{MHz}, \mathrm{CDCl}_{3}\right) 5.5\left(\underline{\mathrm{CH}}_{3} \mathrm{CH}_{2}\right), 19.0\left(\underline{\mathrm{CH}_{3} \mathrm{CH}}\right), 19.5$ $\left(\underline{\mathrm{C}} \mathrm{H}_{3} \mathrm{CH}\right), 43.8\left(\underline{\mathrm{CH}}\left(\mathrm{CH}_{3}\right)_{2}\right), 47.1(\underline{\mathrm{C}}), 48.9\left(\underline{\mathrm{C}}{ }_{2} \mathrm{~N}\right), 49.3\left(\underline{\mathrm{CH}}_{2} \mathrm{SO}_{2}\right), 56.0\left(\underline{\mathrm{CH}}_{3} \mathrm{O}\right), 56.7$ $\left(\underline{\left.\mathrm{CH}_{3} \mathrm{O}\right),} 66.5(\underline{\mathrm{CHSO}}), 102.0(\underline{\mathrm{CHCOCH}}), 103.6(\mathrm{CHCOC} H), 163.4\left(\underline{\mathrm{C}}\left(\mathrm{OCH}_{3}\right)\right), 166.9\right.$

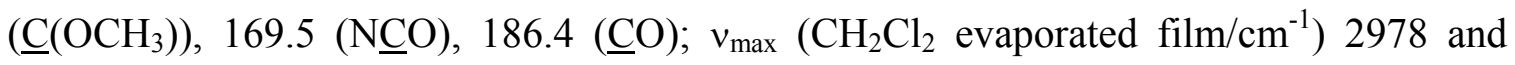
$2943(\mathrm{CH}), 1695(\mathrm{C}=\mathrm{O}), 1661$ (lactam $\mathrm{C}=\mathrm{O}), 1626$ and $1601(\mathrm{C}=\mathrm{C}) ; \mathrm{m} / \mathrm{z}\left(\mathrm{ES}^{+}\right) 380$ $(100 \%, \mathrm{M}+\mathrm{Na}), 262(12 \%), 246(12 \%), 238(10 \%) ; \mathrm{C}_{16} \mathrm{H}_{23} \mathrm{NO}_{6} \mathrm{SNa}(\mathrm{M}+\mathrm{Na})$ found 380.1161, expected 380.1138; mp (EtOAc/petroleum ether) $176.3-178.8^{\circ} \mathrm{C}$. 


\section{rac-(4R,5R)-4-(1H,1H,2H,2H-Heptadecafluorodecylsulfonyl)-2-iso-propyl-6-}

methoxy-2-aza-spiro-[4.5]-deca-9-diene-3,8-dione 32
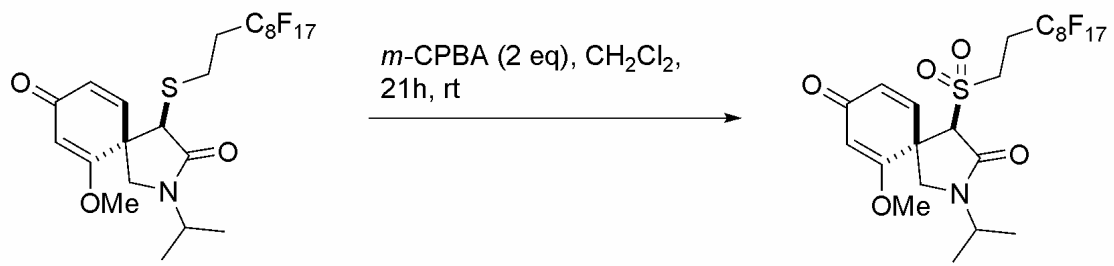

m-CPBA (298 mg, $1.40 \mathrm{mmol}, 2 \mathrm{eq})$ was added to sulfide anti-19 (500 mg, $0.702 \mathrm{mmol}$, 1 eq) in $\mathrm{CH}_{2} \mathrm{Cl}_{2}(20 \mathrm{ml})$. The reaction mixture was stirred at room temperature for 21 hours then washed with aqueous saturated $\mathrm{NaHCO}_{3}$ solution $(3 \times 20 \mathrm{ml})$. The organic layer was dried $\left(\mathrm{Na}_{2} \mathrm{SO}_{4}\right)$ and concentrated in vacuo to give the crude sulfone as a 30:1 anti:syn mixture of diastereoisomers, as a yellow foam. Purification by flash column chromatography on silica gel (50\% ethyl acetate/petroleum ether) afforded spirocyclic sulfone 32 as a >10:1 anti:syn mixture of diastereoisomers, as a cream solid (379 mg, $0.509 \mathrm{mmol}, 73 \%)$.

$\delta_{\mathrm{H}}(500 \mathrm{MHz}, \mathrm{CDCl} 3) 1.19\left(3 \mathrm{H}, \mathrm{d}, J=6.6 \mathrm{~Hz}, \mathrm{CH}_{3} \mathrm{CH}\right), 1.23(3 \mathrm{H}, \mathrm{d}, J=6.9 \mathrm{~Hz}$, $\left.\mathrm{C}_{3} \mathrm{CH}\right), 2.57-2.79$ (2H, m, $\left.\mathrm{CH}_{2} \underline{\mathrm{C}}_{2} \mathrm{C}_{8} \mathrm{~F}_{17}\right), 3.33$ (1H, d, J = 9.8 Hz, CㅂHN), 3.51-3.59 $\left(1 \mathrm{H}, \mathrm{m}, \mathrm{CHHSO}_{2}\right), 3.62(1 \mathrm{H}, \mathrm{d}, J=9.8 \mathrm{~Hz}, \mathrm{CH} \underline{\mathrm{HN}}), 3.85\left(3 \mathrm{H}, \mathrm{s}, \mathrm{CH}_{3} \mathrm{O}\right), 3.92-4.00$ $\left(1 \mathrm{H}, \mathrm{m}, \mathrm{CH}_{\underline{H} S \mathrm{~S}_{2}}\right), 4.46\left(1 \mathrm{H}\right.$, apparent septet, $\left.J=6.7 \mathrm{~Hz}, \underline{\mathrm{C}}\left(\mathrm{CH}_{3}\right)_{2}\right), 4.47(1 \mathrm{H}, \mathrm{s}$, $\left.\mathrm{C}^{\mathrm{HSO}}{ }_{2}\right), 5.77(1 \mathrm{H}, \mathrm{d}, J=1.6 \mathrm{~Hz}, \mathrm{C} \underline{\mathrm{HCOCH}}=\mathrm{CH}), 6.39(1 \mathrm{H}, \mathrm{dd}, J=10.1 \mathrm{~Hz}, 1.6 \mathrm{~Hz}$, $\mathrm{CHCOC} \underline{\mathrm{H}}=\mathrm{CH}), 7.14(1 \mathrm{H}, \mathrm{d}, J=10.1 \mathrm{~Hz}, \mathrm{CHCOCH}=\mathrm{C} \underline{\mathrm{H}}) ; \delta_{\mathrm{C}}\left(125 \mathrm{MHz}, \mathrm{CDCl}_{3}\right) 19.4$

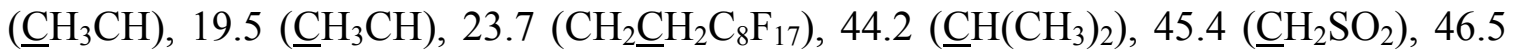

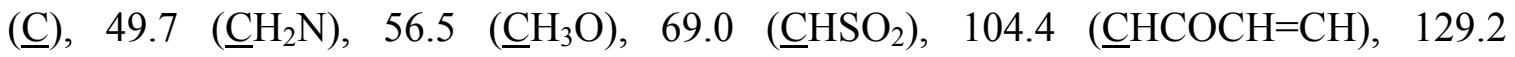

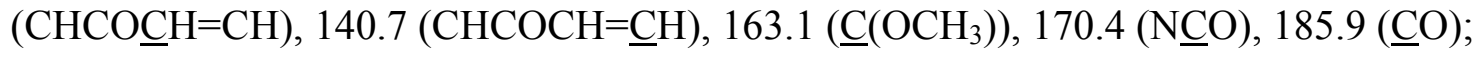
$v_{\max }\left(\mathrm{CH}_{2} \mathrm{Cl}_{2}\right.$ evaporated film/ $\left./ \mathrm{cm}^{-1}\right) 2981$ and $2930(\mathrm{CH}), 1696(\mathrm{C}=\mathrm{O}), 1662$ (lactam $\mathrm{C}=\mathrm{O}), \quad 1603 \quad(\mathrm{C}=\mathrm{C}) ; \quad \mathrm{m} / \mathrm{z} \quad\left(\mathrm{ES}^{+}\right) 768 \quad(100 \%, \mathrm{M}+\mathrm{Na}), 457 \quad(50 \%), 441$ (37\%); $\mathrm{C}_{23} \mathrm{H}_{20} \mathrm{NO}_{5} \mathrm{~F}_{17} \mathrm{SNa}$ found 768.0675 , expected 768.0683; mp (MeOH) $128.8-130.5^{\circ} \mathrm{C}$.

Treatment of sulfide 19 as a 2:1 anti:syn mixture also afforded anti-sulfone 32 as a $>12: 1$ mixture of diastereoisomers, $85 \%$ yield, after flash column chromatography on silica gel. 
rac-(4R,5R)-4-(1H,1H,2H,2H-Heptadecafluorodecylsulfonyl)-2-iso-propyl-6methoxy-2-aza-spiro-[4.5]-deca,6-ene-3,8-dione 33

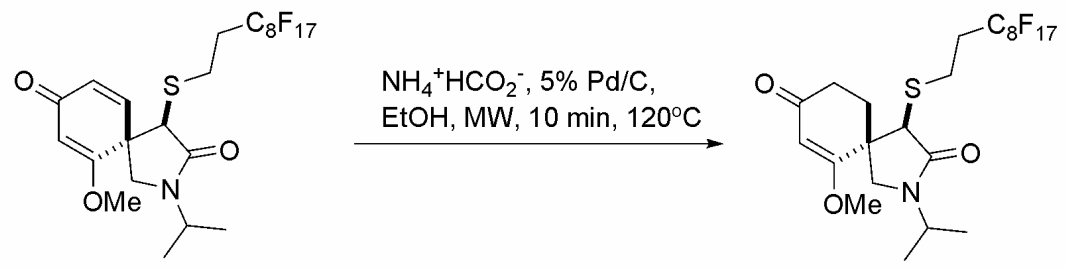

Dienone 32 (77 mg, $0.103 \mathrm{mmol}, 1 \mathrm{eq})$, ammonium formate (30 mg, $0.310 \mathrm{mmol}, 3 \mathrm{eq}$ ), $5 \% \mathrm{Pd} / \mathrm{C}(10 \mathrm{mg}, 5 \mathrm{~mol} \%)$ and $\mathrm{EtOH}(1.5 \mathrm{ml})$ were added to a microwave vial equipped with a magnetic stirrer. The capped vial was purged with $\mathrm{N}_{2}$ for one minute. The reaction mixture was subjected to microwave irradiation (high absorption) at $120{ }^{\circ} \mathrm{C}$ for 10 minutes. After cooling the reaction mixture was immediately filtered through celite, washing with ethyl acetate and the solvent was removed in vacuo to afford enone $\mathbf{3} 3$ as a colourless oil (76 mg, $0.102 \mathrm{mmol}, 98 \%$ ). No further purification was required.

$\delta_{\mathrm{H}}\left(500 \mathrm{MHz}, \mathrm{CDCl}_{3}\right) 1.17\left(3 \mathrm{H}, \mathrm{d}, J=6.9 \mathrm{~Hz}, \underline{\mathrm{C}}_{3} \mathrm{CH}\right), 1.21(3 \mathrm{H}, \mathrm{d}, J=6.6 \mathrm{~Hz}$, $\left.\mathrm{C}_{3} \mathrm{CH}\right), 2.26-2.33(1 \mathrm{H}, \mathrm{m}, \mathrm{C} \underline{\mathrm{H}}), 2.42-2.49(1 \mathrm{H}, \mathrm{m}, \mathrm{C} \underline{\mathrm{H}}), 2.57-2.88(3 \mathrm{H}, \mathrm{m}$, $\mathrm{C}_{2} \mathrm{C}_{8} \mathrm{~F}_{17}$ and $\left.\mathrm{CH} \underline{\mathrm{H}}\right), 2.85-2.91(1 \mathrm{H}, \mathrm{m}, \mathrm{CH} \underline{\mathrm{H}}), 3.41(1 \mathrm{H}, \mathrm{d}, J=9.8 \mathrm{~Hz}, \mathrm{C} \underline{H} H \mathrm{~N}), 3.59-$

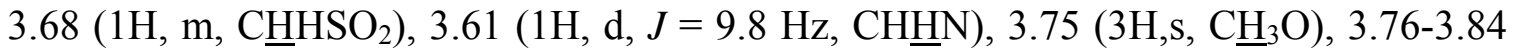
$\left(1 \mathrm{H}, \mathrm{m}, \mathrm{CH}_{\underline{H}} \mathrm{SO}_{2}\right), 4.25(1 \mathrm{H}, \mathrm{s}, \mathrm{C} \underline{\mathrm{H}} \mathrm{S}), 4.42\left(1 \mathrm{H}\right.$, apparent septet, $\left.J=6.8 \mathrm{~Hz}, \mathrm{C} \underline{\mathrm{H}}\left(\mathrm{CH}_{3}\right)_{2}\right)$, $5.45(1 \mathrm{H}, \mathrm{s}, \mathrm{C} \underline{\mathrm{HCO}}) ; \delta_{\mathrm{C}}\left(125 \mathrm{MHz}, \mathrm{CDCl}_{3}\right) 19.3\left(\underline{\mathrm{CH}}_{3} \mathrm{CH}\right), 19.4\left(\underline{\mathrm{CH}}_{3} \mathrm{CH}\right), 26.1\left(\mathrm{t}, J^{2}{ }_{\mathrm{CF}}=\right.$

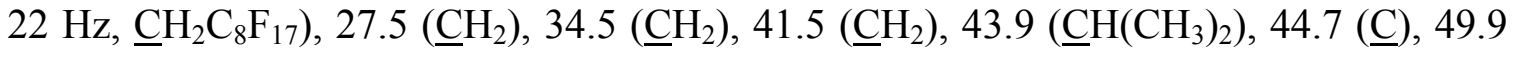
$\left(\underline{\mathrm{C}} \mathrm{H}_{2} \mathrm{~N}\right), 56.7\left(\underline{\mathrm{CH}}_{3} \mathrm{O}\right), 68.1 \quad(\underline{\mathrm{CHSO}}), 103.1(\mathrm{C}=\underline{\mathrm{CHCO}}), 164.2\left(\underline{\mathrm{C}}\left(\mathrm{OCH}_{3}\right)\right), 175.2$ (NCO), 196.9 (ㅁ); $v_{\max }\left(\mathrm{CH}_{2} \mathrm{Cl}_{2}\right.$ evaporated film/ $\left./ \mathrm{cm}^{-1}\right) 2980$ and $2947(\mathrm{CH}), 1695$ $(\mathrm{C}=\mathrm{O}), 1659$ (lactam $\mathrm{C}=\mathrm{O}), 1608(\mathrm{C}=\mathrm{C}) ; \mathrm{m} / \mathrm{z}\left(\mathrm{ES}^{+}\right) 770(42 \%, \mathrm{M}+\mathrm{Na}), 458$ (12\%); $\mathrm{C}_{23} \mathrm{H}_{22} \mathrm{NO}_{5} \mathrm{~F}_{17} \mathrm{SNa}(\mathrm{M}+\mathrm{Na})$ found 770.0846 , expected 770.0839 . 
rac-4-(1H,1H,2H,2H-Heptadecafluorodecylsulfonyl)-2-iso-propyl-6,10-dimethoxy-4methyl-2-aza-spiro-[4.5]-deca,6,9-diene-3,8-dione 34

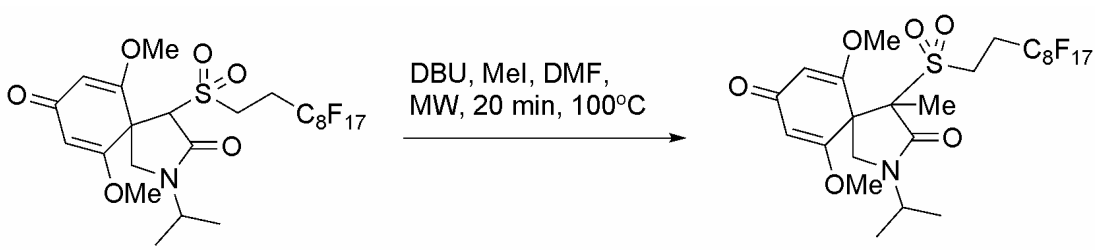

Sulfone 30 (62 mg, $0.080 \mathrm{mmol}, 1 \mathrm{eq})$, DMF (1.5 ml), DBU (0.06 ml, $0.40 \mathrm{mmol}, 5 \mathrm{eq})$ and $\mathrm{MeI}(0.03 \mathrm{ml}, 0.40 \mathrm{mmol}, 5 \mathrm{eq})$ were added to a microwave vial equipped with a magnetic stirrer and the vial was capped. The reaction mixture was subjected to microwave irradiation (high absorption) at $100{ }^{\circ} \mathrm{C}$ for 10 minutes. After cooling, DBU $(0.06 \mathrm{ml}, 0.40 \mathrm{mmol}, 5 \mathrm{eq})$ and $\mathrm{MeI}(0.03 \mathrm{ml}, 0.40 \mathrm{mmol}, 5 \mathrm{eq})$ were added and the reaction mixture was subjected to a second period of microwave irradiation (high absorption) at $100{ }^{\circ} \mathrm{C}$ for 10 minutes. After cooling the reaction mixture was diluted with ethyl acetate $(1 \mathrm{ml})$ and washed with water $(3 \times 2 \mathrm{ml})$, the organic layer was dried $\left(\mathrm{Na}_{2} \mathrm{SO}_{4}\right)$ and concentrated in vacuo to give an orange oil. Purification by flash column chromatography on silica gel (50\% ethyl acetate/petroleum ether) afforded 34 as a colourless oil (35 mg, $0.044 \mathrm{mmol}, 55 \%)$.

$\delta_{\mathrm{H}}\left(500 \mathrm{MHz}, \mathrm{CDCl}_{3}\right) 1.20\left(3 \mathrm{H}, \mathrm{d}, J=6.6 \mathrm{~Hz}, \mathrm{CH}_{3} \mathrm{CH}\right), 1.25(3 \mathrm{H}, \mathrm{d}, J=6.9 \mathrm{~Hz}$, $\left.\mathrm{C}_{3} \mathrm{CH}\right), 1.45\left(3 \mathrm{H}, \mathrm{s}, \mathrm{CH}_{3} \mathrm{C}\right), 2.48-2.70\left(2 \mathrm{H}, \mathrm{m}, \mathrm{C}_{2} \mathrm{C}_{8} \mathrm{~F}_{17}\right), 3.36(1 \mathrm{H}, \mathrm{ddd}, J=12.6 \mathrm{~Hz}$, $\left.12.3 \mathrm{~Hz}, 4.4 \mathrm{~Hz}, \mathrm{C}_{\mathbf{H} H S O}\right), 3.44(1 \mathrm{H}, \mathrm{d}, J=9.8 \mathrm{~Hz}, \mathrm{C} \underline{\mathrm{HHN}}), 3.68\left(3 \mathrm{H}, \mathrm{s}, \mathrm{C}_{3} \mathrm{O}\right), 3.73$ $\left(3 \mathrm{H}, \mathrm{s}, \mathrm{CH}_{3} \mathrm{O}\right), 3.84(1 \mathrm{H}, \mathrm{ddd}, J=12.6 \mathrm{~Hz}, 12.6 \mathrm{~Hz}, 5.0 \mathrm{~Hz}), 4.26(1 \mathrm{H}, \mathrm{d}, J=9.8 \mathrm{~Hz}$, CHㅍN), $4.47\left(1 \mathrm{H}\right.$, apparent septet, $\left.J=6.7 \mathrm{~Hz}, \mathrm{C} \underline{\mathrm{H}}\left(\mathrm{CH}_{3}\right)_{2}\right), 5.60(1 \mathrm{H}, \mathrm{s}, \mathrm{C} \underline{\mathrm{HCOCH}}), 5.66$

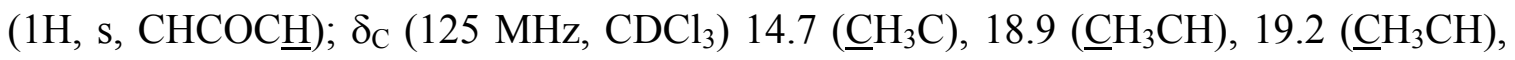
$23.8\left(\underline{\mathrm{CH}}_{2} \mathrm{C}_{8} \mathrm{~F}_{17}, \mathrm{t}, J=23 \mathrm{~Hz}\right), 43.9\left(\underline{\mathrm{CH}}_{2}\right), 44.4\left(\underline{\mathrm{CH}}\left(\mathrm{CH}_{3}\right)_{2}\right), 45.0\left(\underline{\mathrm{CH}_{2}}\right), 52.6(\underline{\mathrm{C}}), 56.1$ $\left(\underline{\mathrm{C}} \mathrm{H}_{3} \mathrm{O}\right), 56.2\left(\underline{\mathrm{CH}}_{3} \mathrm{O}\right), 76.5\left(\underline{\mathrm{C}}\left(\mathrm{CH}_{3}\right) \mathrm{SO}_{2}\right), 103.4(\underline{\mathrm{CHCOCH}}), 104.4(\mathrm{CHCO} \underline{\mathrm{H}}), 165.8$ $\left(\underline{\mathrm{C}}\left(\mathrm{OCH}_{3}\right)\right), 166.4\left(\underline{\mathrm{C}}\left(\mathrm{OCH}_{3}\right)\right), 169.2(\mathrm{NCO}), 186.3(\underline{\mathrm{CO}}) ; v_{\max }\left(\mathrm{CH}_{2} \mathrm{Cl}_{2}\right.$ evaporated film/cm $\left.\mathrm{cm}^{-1}\right) 2941$ and $2979(\mathrm{CH}), 1695(\mathrm{C}=\mathrm{O}), 1658$ (lactam $\left.\mathrm{C}=\mathrm{O}\right), 1594(\mathrm{C}=\mathrm{C}) ; \mathrm{m} / \mathrm{z}\left(\mathrm{ES}^{+}\right)$ $812(\mathrm{M}+\mathrm{Na}, 100 \%), 807\left(\mathrm{M}+\mathrm{NH}_{4}^{+}, 38 \%\right) ; \mathrm{C}_{25} \mathrm{H}_{25} \mathrm{NO}_{6} \mathrm{SF}_{17}\left(\mathrm{MH}^{+}\right)$found 790.1131, expected 790.1126 . 
rac-(4R,5R,8S,10R)-4-(1H,1H,2H,2H-Heptadecafluoro decylsulfanyl)-8-hydroxy-2iso-propyl-6,10-dimethoxy-2-aza spiro-[4.5]-deca-6-ene-3-one 36 rac-(4R,5R,10R)-4-(1H,1H,2H,2H perfluorodecylsulfanyl)-2-iso-propyl-10-methoxy2-aza spiro-[4.5]-deca-7-ene-3,6-dione 38

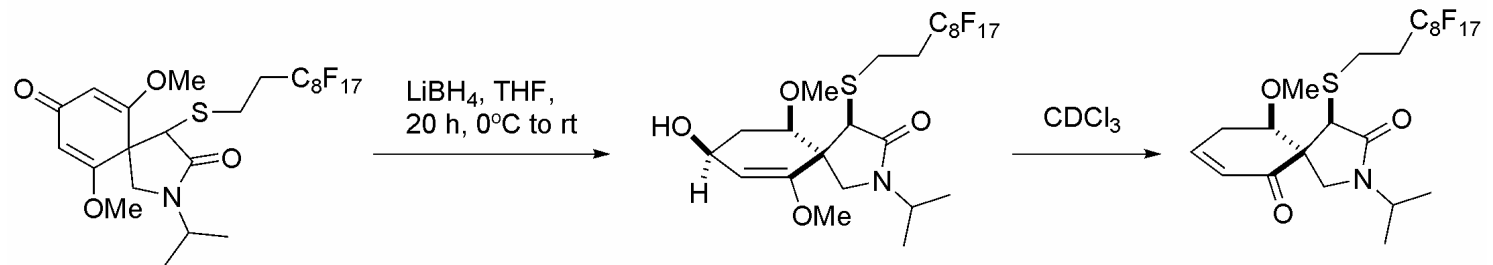

$\mathrm{LiBH}_{4}$ (12 mg, $\left.0.57 \mathrm{mmol}, 6 \mathrm{eq}\right)$ was added to spirocyclic dieneone 11 (71 mg, 0.096 $\mathrm{mmol}, 1 \mathrm{eq}$ ) in $\mathrm{THF}$ at $0{ }^{\circ} \mathrm{C}$. The mixture was stirred at room temperature for 20 hours, during which time the solution became orange in colour. The solvent was removed in vacuo and the residual orange solid was taken up in $\mathrm{H}_{2} \mathrm{O}(4 \mathrm{ml})$ and the solution was extracted with EtOAc $(5 \times 10 \mathrm{ml})$. The combined organic layers were dried $\left(\mathrm{Na}_{2} \mathrm{SO}_{4}\right)$ and concentrated in vacuo to give the crude product. Purification by flash column chromatography (50\% ethyl aetate/petroleum ether) gave 36 as a colourless oil (33 mg, $0.044 \mathrm{mmol}, 46 \%)$.

$\delta_{\mathrm{H}}\left(300 \mathrm{MHz}, \mathrm{CDCl}_{3}\right) 1.11\left(3 \mathrm{H}, \mathrm{d}, J=6.7 \mathrm{~Hz}, \underline{\mathrm{CH}}_{3} \mathrm{CH}\right), 1.12(3 \mathrm{H}, \mathrm{d}, J=6.9 \mathrm{~Hz}$, $\left.\mathrm{C}_{3} \mathrm{CH}\right), 1.78-1.93\left(1 \mathrm{H}, \mathrm{m}, \mathrm{C} \underline{\mathrm{HHCH}}\left(\mathrm{OCH}_{3}\right)\right), 2.35$ (1H, m, $\left.\mathrm{CH} \underline{\mathrm{HCH}}\left(\mathrm{OCH}_{3}\right)\right), 2.45-2.69$ $\left(2 \mathrm{H}, \mathrm{m}, \mathrm{C}_{2} \mathrm{C}_{8} \mathrm{~F}_{17}\right), 2.93-3.13\left(2 \mathrm{H}, \mathrm{m}, \mathrm{C}_{2} \mathrm{CH}_{2} \mathrm{C}_{8} \mathrm{~F}_{17}\right), 3.16-3.25$ (2H, m, C$H \mathrm{HN}$ and $\mathrm{C} \underline{\mathrm{H}}\left(\mathrm{OCH}_{3}\right), 3.37(1 \mathrm{H}, \mathrm{d}, J=9.1 \mathrm{~Hz}, \mathrm{CH} \underline{\mathrm{HN}}), 3.42\left(3 \mathrm{H}, \mathrm{s}, \mathrm{C}_{3} \mathrm{O}\right), 3.47\left(3 \mathrm{H}, \mathrm{s}, \mathrm{C}_{3} \mathrm{O}\right)$, $3.83(1 \mathrm{H}, \mathrm{s}, \mathrm{C} \underline{\mathrm{HS}}), 4.28-4.45\left(2 \mathrm{H}, \mathrm{m}, \underline{\mathrm{CH}}\left(\mathrm{CH}_{3}\right)_{2}\right.$ and $\left.\mathrm{CH}(\mathrm{O} \underline{\mathrm{H}})\right), 4.84(1 \mathrm{H}, \mathrm{d}, J=2.6 \mathrm{~Hz}$, $=\mathrm{C} \underline{H C H}(\mathrm{OH})) ; \quad \delta_{\mathrm{C}} \quad\left(75 \quad \mathrm{MHz}, \quad \mathrm{CDCl}_{3}\right) \quad 19.3 \quad\left(\underline{\mathrm{CH}}_{3} \mathrm{CH}\right), \quad 19.7 \quad\left(\underline{\mathrm{CH}}_{3} \mathrm{CH}\right), \quad 24.0$

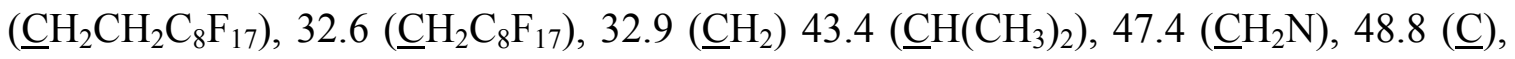
$49.3(\underline{\mathrm{C} H S}), 54.7\left(\underline{\left.\mathrm{CH}_{3} \mathrm{O}\right),} 57.3\left(\underline{\mathrm{CH}}_{3} \mathrm{O}\right), 65.2(\underline{\mathrm{CH}}(\mathrm{OH})), 78.9\left(\underline{\mathrm{CH}}\left(\mathrm{OCH}_{3}\right)\right), 101.4\right.$ $(\underline{\mathrm{CH}}=\mathrm{CHOH}), 156.2\left(\mathrm{CH}=\underline{\mathrm{C}}\left(\mathrm{OCH}_{3}\right)\right), 171.8(\mathrm{NCO}) ; v_{\max }\left(\mathrm{CH}_{2} \mathrm{Cl}_{2}\right.$ evaporated film/cm $\left.{ }^{-1}\right)$ 3419 (broad, OH), 2976 and $2932(\mathrm{CH}), 1694$ (lactam C=O); m/z (ES $) 770(\mathrm{M}+\mathrm{Na}$, 100\%), $748\left(\mathrm{MH}^{+}, 33 \%\right) ; \mathrm{C}_{24} \mathrm{H}_{26} \mathrm{NO}_{4} \mathrm{~F}_{17} \mathrm{SNa}(\mathrm{M}+\mathrm{Na})$ found 770.1211, expected 770.1203 . 
On standing in $\mathrm{CDCl}_{3} 36$ was found to undergo elimination to afford enone 38 as a yellow oil.

$\delta_{\mathrm{H}}\left(500 \mathrm{MHz}, \mathrm{CDCl}_{3}\right) 1.16\left(3 \mathrm{H}, \mathrm{d}, J=6.9 \mathrm{~Hz}, \underline{\mathrm{CH}}_{3} \mathrm{CH}\right), 1.22(3 \mathrm{H}, \mathrm{d}, J=6.9 \mathrm{~Hz}$, $\left.\mathrm{C}_{3} \mathrm{CH}\right), 2.31-2.45\left(2 \mathrm{H}, \mathrm{m}, \mathrm{C}_{2} \mathrm{C}_{8} \mathrm{~F}_{17}\right), 2.49$ (1H, ddt, $J=18.7 \mathrm{~Hz}, 9.5 \mathrm{~Hz}, 2.8 \mathrm{~Hz}$, $\left.\mathrm{CHC} \underline{H} H C H\left(\mathrm{OCH}_{3}\right)\right), 2.73-2.88\left(2 \mathrm{H}, \mathrm{m}, \mathrm{C}_{2} \mathrm{CH}_{2} \mathrm{C}_{8} \mathrm{~F}_{17}\right), 2.90(1 \mathrm{H}, \mathrm{dt}, J=18.7 \mathrm{~Hz}, 5.0$ $\left.\mathrm{Hz}, \mathrm{CHCH} \underline{\mathrm{HCH}}\left(\mathrm{OCH}_{3}\right)\right), 3.13(1 \mathrm{H}, \mathrm{d}, J=9.8 \mathrm{~Hz}, \mathrm{C} \underline{\mathrm{HHN}}), 3.41\left(3 \mathrm{H}, \mathrm{s}, \mathrm{C}_{3} \mathrm{O}\right), 3.60(1 \mathrm{H}$, dd, $\left.J=9.5 \mathrm{~Hz}, 5.0 \mathrm{~Hz}, \mathrm{CH}_{2} \mathrm{C} \underline{\mathrm{H}}\left(\mathrm{OCH}_{3}\right)\right), 3.79(1 \mathrm{H}, \mathrm{s}, \mathrm{C} \underline{\mathrm{HS}}), 4.07$ (1H, d, $J=9.8 \mathrm{~Hz}$, CHㅍN), $4.40\left(1 \mathrm{H}\right.$, septet, $\left.J=6.9 \mathrm{~Hz}, \underline{\mathrm{CH}}\left(\mathrm{CH}_{3}\right)_{2}\right), 6.19(1 \mathrm{H}, \mathrm{dd}, J=10.1 \mathrm{~Hz}, 2.8 \mathrm{~Hz}$, $\mathrm{COC} \underline{\mathrm{H}}=\mathrm{CH}), 6.85-6.91(1 \mathrm{H}, \mathrm{m}, \mathrm{COCH}=\mathrm{C} \underline{\mathrm{H}}) ; \delta_{\mathrm{C}}\left(125 \mathrm{MHz}, \mathrm{CDCl}_{3}\right) 19.2\left(\underline{\mathrm{CH}_{3} \mathrm{CH}}\right), 19.6$

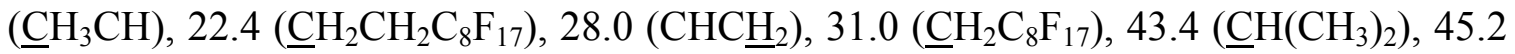
$(\underline{\mathrm{CHS}}), 45.5\left(\underline{\mathrm{CH}_{2} \mathrm{~N}}\right), 55.6(\underline{\mathrm{C}}), 57.2\left(\underline{\mathrm{CH}}_{3} \mathrm{O}\right), 80.7\left(\underline{\mathrm{CH}}\left(\mathrm{OCH}_{3}\right) 130.4(\mathrm{CO} \underline{\mathrm{CH}}=\mathrm{CH}), 144.9\right.$ $(\mathrm{COCH}=\underline{\mathrm{CH}}), 168.8(\mathrm{NCO}), 195.7(\underline{\mathrm{CO}}) ; v_{\max }\left(\mathrm{CH}_{2} \mathrm{Cl}_{2}\right.$ evaporated film/cm $\left.{ }^{-1}\right) 2977$ and $2936(\mathrm{CH}), 1685(\mathrm{C}=\mathrm{O}), 1608(\mathrm{C}=\mathrm{C}) ; \mathrm{m} / \mathrm{z}\left(\mathrm{ES}^{+}\right) 738$ (100\%, M+Na), 418 (39\%); $\mathrm{C}_{23} \mathrm{H}_{22} \mathrm{NO}_{3} \mathrm{~F}_{17} \mathrm{SNa}(\mathrm{M}+\mathrm{Na})$ found 738.0936, expected 738.0941.

rac-(4R,5R,8S,10R)-4-Decylsulfanyl-8-hydroxy-2-iso-propyl-6,10-dimethoxy-2-aza spiro-[4.5]-deca-6-ene-3-one 37
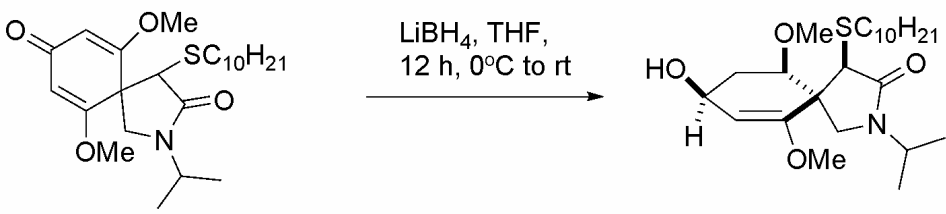

$\mathrm{LiBH}_{4}(30 \mathrm{mg}, 1.38 \mathrm{mmol}, 6 \mathrm{eq})$ was added to dienone 35 (100 mg, $\left.0.230 \mathrm{mmol}, 1 \mathrm{eq}\right)$ in THF $(10 \mathrm{ml})$ at $0{ }^{\circ} \mathrm{C}$. The reaction mixture was stirred at room temperature for 12 hours, during which time the solution became orange. $\mathrm{MeOH}(5 \mathrm{ml})$ was added and the solvents were removed in vacuo. The orange residue was taken up in $\mathrm{H}_{2} \mathrm{O}(10 \mathrm{ml})$ and the solution was extracted with EtOAc $(5 \times 10 \mathrm{ml})$. The combined organic layers were dried $\left(\mathrm{Na}_{2} \mathrm{SO}_{4}\right)$ and concentrated in vacuo to afford 37 as a colourless oil $(89 \mathrm{mg}, 0.201 \mathrm{mmol}$, $88 \%$ ). No further purification was required. 
$\delta_{\mathrm{H}}\left(500 \mathrm{MHz}, \mathrm{CDCl}_{3}\right) 0.88\left(3 \mathrm{H}, \mathrm{t}, J=6.9 \mathrm{~Hz}, \mathrm{C}_{3} \mathrm{CH}_{2}\right), 1.12(3 \mathrm{H}, \mathrm{d}, J=6.6 \mathrm{~Hz}$, $\left.\mathrm{C}_{3} \mathrm{CH}\right), 1.13\left(3 \mathrm{H}, \mathrm{d}, J=6.9 \mathrm{~Hz}, \underline{\mathrm{C}}_{3} \mathrm{CH}\right), 1.20-1.33\left(12 \mathrm{H}, \mathrm{m}, 6 \times \mathrm{C}_{2}\right), 1.33-1.41(2 \mathrm{H}$, $\left.\mathrm{m}, \mathrm{C}_{2}\right), 1.54-1.67\left(2 \mathrm{H}, \mathrm{m}, \mathrm{C}_{2}\right), 1.89(1 \mathrm{H}, \mathrm{ddd}, J=12.2 \mathrm{~Hz}, 12.0 \mathrm{~Hz}, 9.1 \mathrm{~Hz}$, $\left.\mathrm{C} \underline{H H C H}\left(\mathrm{OCH}_{3}\right)\right), 2.46\left(1 \mathrm{H}\right.$, ddd, $\left.J=12.0 \mathrm{~Hz}, 6.7 \mathrm{~Hz}, 3.5 \mathrm{~Hz}, \mathrm{CH} \underline{\mathrm{HC}}\left(\mathrm{OCH}_{3}\right)\right), 2.79-2.92$ $\left(2 \mathrm{H}, \mathrm{m}, \underline{\mathrm{C}}_{2} \mathrm{~S}\right), 3.19\left(1 \mathrm{H}, \mathrm{dd}, J=12.2 \mathrm{~Hz}, 3.5 \mathrm{~Hz}, \mathrm{C} \underline{\mathrm{H}}\left(\mathrm{OCH}_{3}\right)\right), 3.20(1 \mathrm{H}, \mathrm{d}, J=8.8 \mathrm{~Hz}$, CㅂHN), $3.36(1 \mathrm{H}, \mathrm{d}, J=8.8 \mathrm{~Hz}, \mathrm{CH} \underline{H N}), 3.42\left(3 \mathrm{H}, \mathrm{s}, \mathrm{C}_{3} \mathrm{O}\right), 3.48\left(3 \mathrm{H}, \mathrm{s}, \mathrm{C}_{3} \mathrm{O}\right), 3.83$ $(1 \mathrm{H}, \mathrm{s}, \mathrm{C} \underline{\mathrm{HS}}), 4.30-4.42\left(2 \mathrm{H}, \mathrm{m}, \mathrm{C} \underline{\mathrm{H}}(\mathrm{OH})\right.$ and $\left.\underline{\mathrm{C}}\left(\mathrm{CH}_{3}\right)_{2}\right), 4.82(1 \mathrm{H}, \mathrm{d}, J=2.8$, $\left.\mathrm{C} \underline{H}=\mathrm{C}\left(\mathrm{OCH}_{3}\right)\right) ; \delta_{\mathrm{C}}\left(125 \mathrm{MHz}, \mathrm{CDCl}_{3}\right) 14.1\left(\underline{\mathrm{CH}}_{3} \mathrm{CH}_{2}\right), 19.1\left(\underline{\mathrm{CH}}_{3} \mathrm{CH}\right), 19.5\left(\underline{\mathrm{C}} \mathrm{H}_{3} \mathrm{CH}\right)$, $\left.22.7\left(\underline{\mathrm{CH}_{2}}\right), 29.1-29.6\left(6 \times \underline{\mathrm{CH}}_{2}\right), 31.9\left(\underline{\mathrm{CH}}_{2}\right), 32.8\left(\underline{\mathrm{CH}}_{2} \mathrm{~S}\right), 32.9(\mathrm{CH}(\mathrm{OH}) \underline{\mathrm{CH}})_{2}\right), 43.0$ $\left(\underline{\mathrm{C}} \mathrm{H}\left(\mathrm{CH}_{3}\right)_{2}\right), 46.5\left(\underline{\mathrm{C}} \mathrm{H}_{2} \mathrm{~N}\right), 47.7(\underline{\mathrm{CHS}}), 48.6(\underline{\mathrm{C}}), 54.5\left(\underline{\mathrm{CH}}_{3} \mathrm{O}\right), 57.1\left(\underline{\left.\mathrm{CH}_{3} \mathrm{O}\right), 65.1}\right.$ $(\underline{\mathrm{C}} \mathrm{H}(\mathrm{OH})), 78.6\left(\underline{\mathrm{CH}}\left(\mathrm{OCH}_{3}\right)\right), 100.7\left(\underline{\mathrm{CH}}=\mathrm{C}\left(\mathrm{OCH}_{3}\right)\right), 156.4\left(\mathrm{CH}=\underline{\mathrm{C}}\left(\mathrm{OCH}_{3}\right)\right), 172.2$ (NCO); $v_{\max }\left(\mathrm{CH}_{2} \mathrm{Cl}_{2}\right.$ evaporated film/ $\left./ \mathrm{cm}^{-1}\right) 3412$ (broad, $\left.\mathrm{OH}\right), 2926$ and $2854(\mathrm{CH})$, 1681 (lactam C=O); $m / z\left(E^{-}\right) 440(100 \%, M-H), 266(39 \%) ; \mathrm{C}_{24} \mathrm{H}_{44} \mathrm{NO}_{4} \mathrm{~S}\left(\mathrm{MH}^{+}\right)$found 442.2981, expected 442.2986. 
NMR spectra : $\alpha$-hydroxyamides

$500 \mathrm{MHz}\left(\mathrm{CDCl}_{3}\right)$

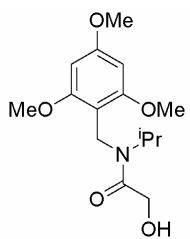

39

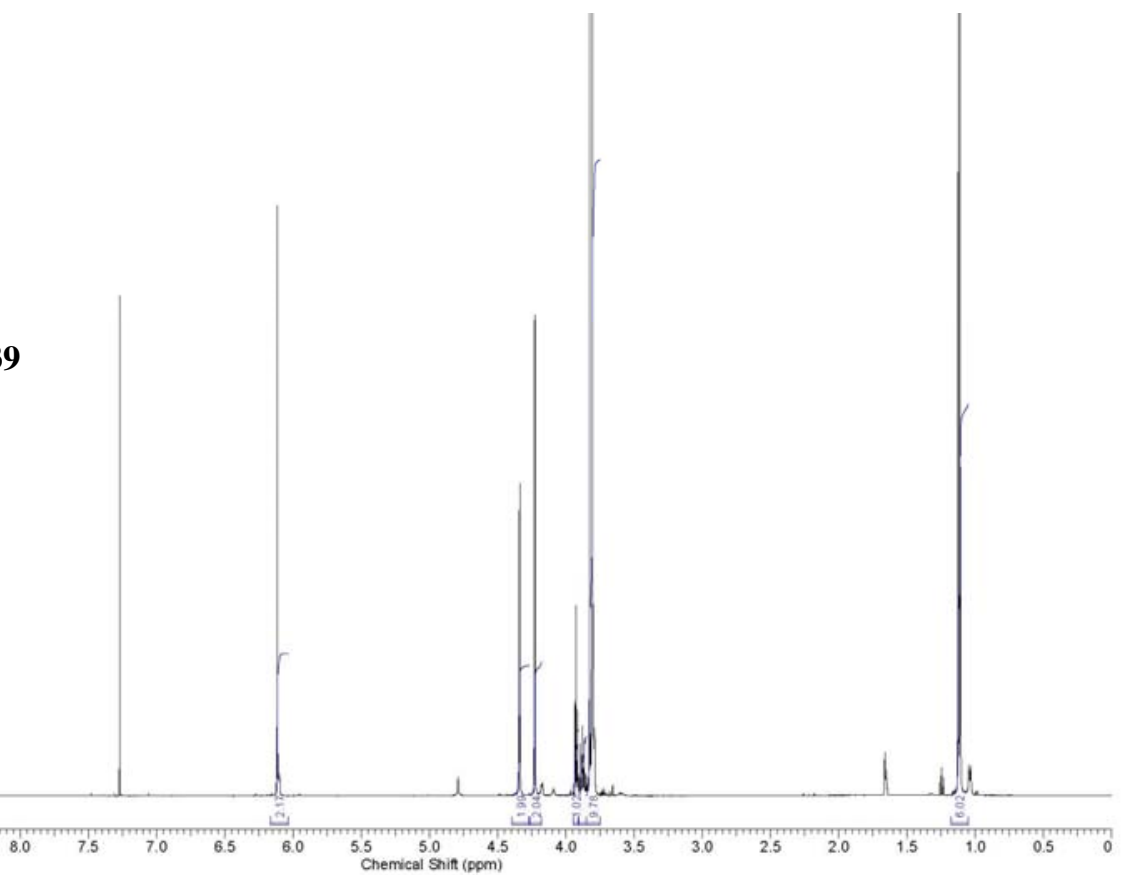

$125 \mathrm{MHz}\left(\mathrm{CDCl}_{3}\right)$

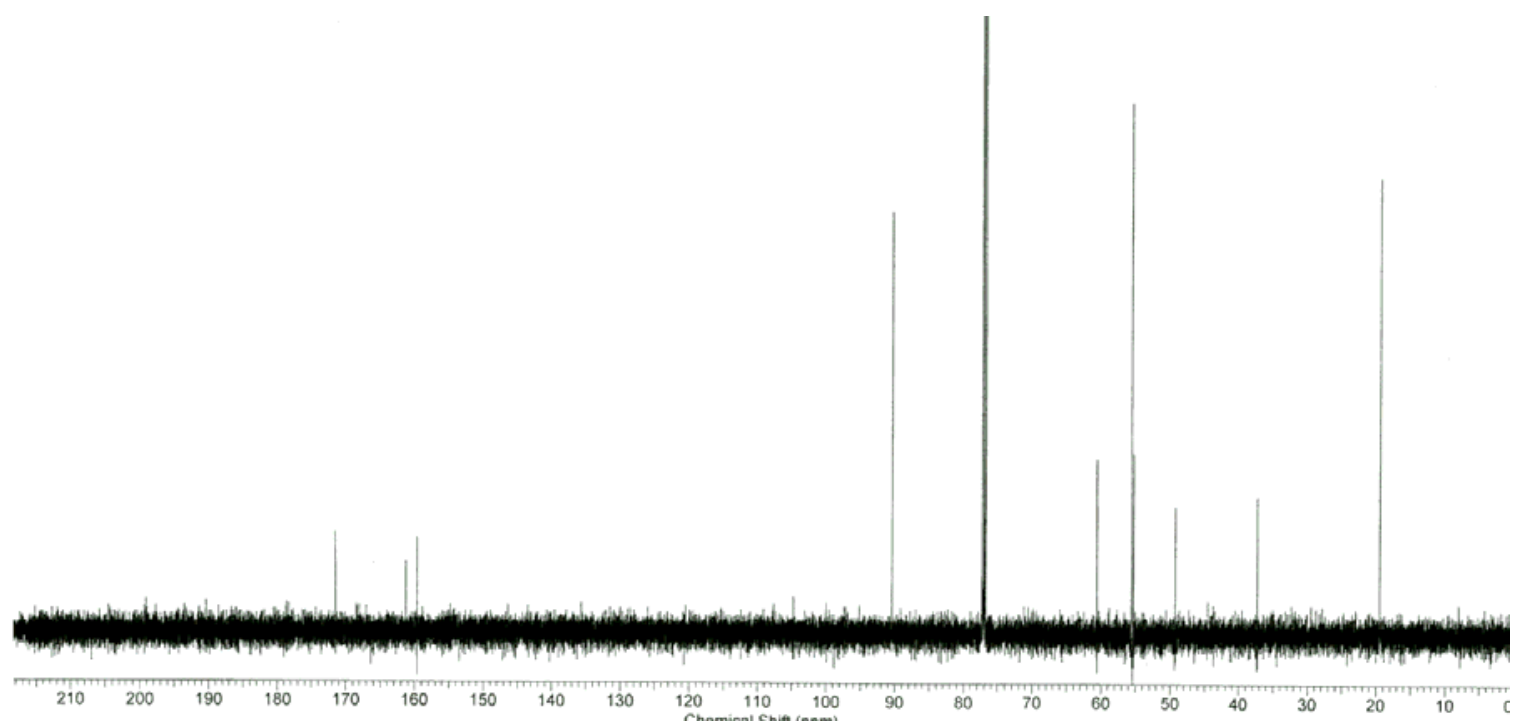


$500 \mathrm{MHz}\left(\mathrm{CDCl}_{3}\right)$

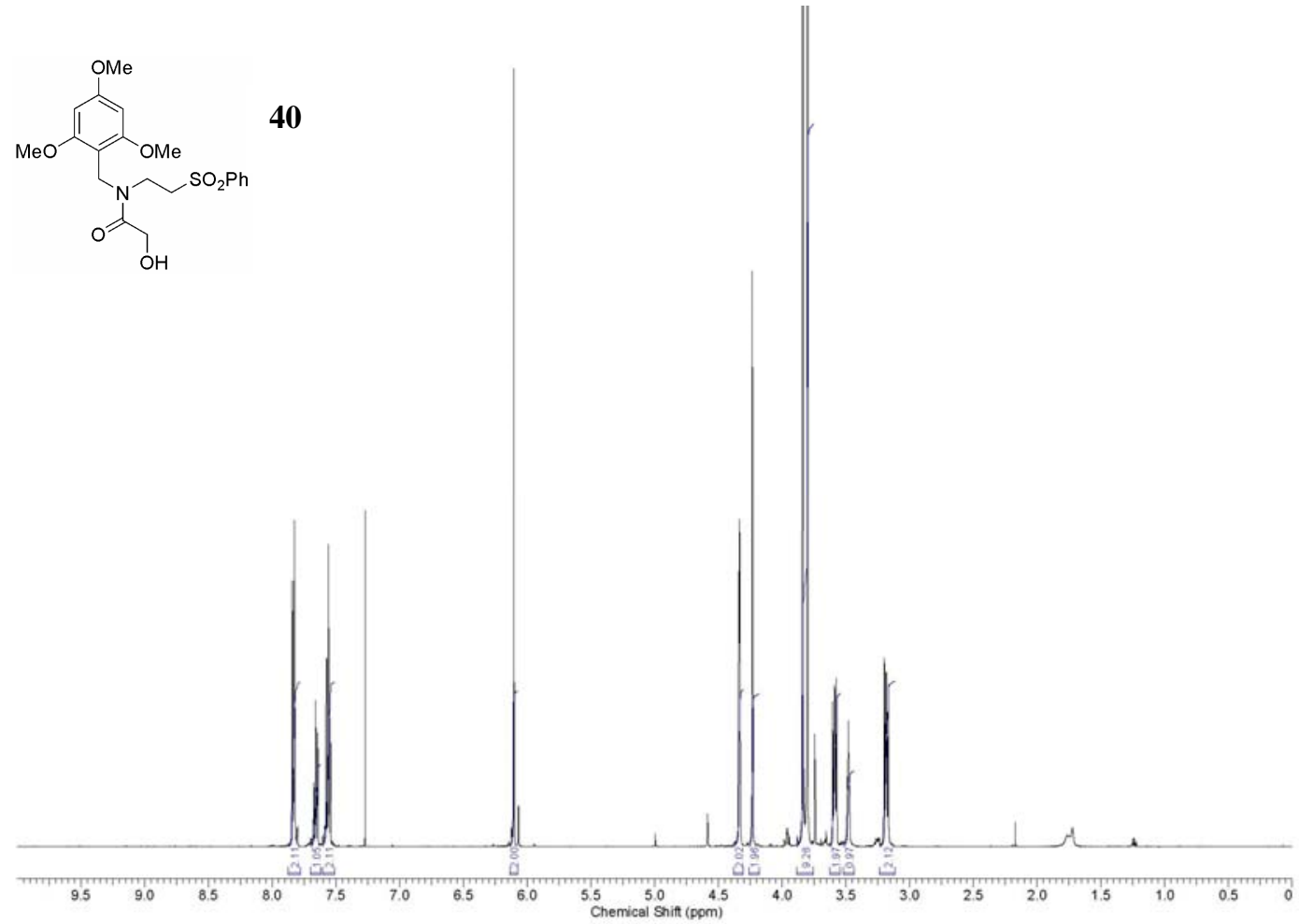

$125 \mathrm{MHz}\left(\mathrm{CDCl}_{3}\right)$

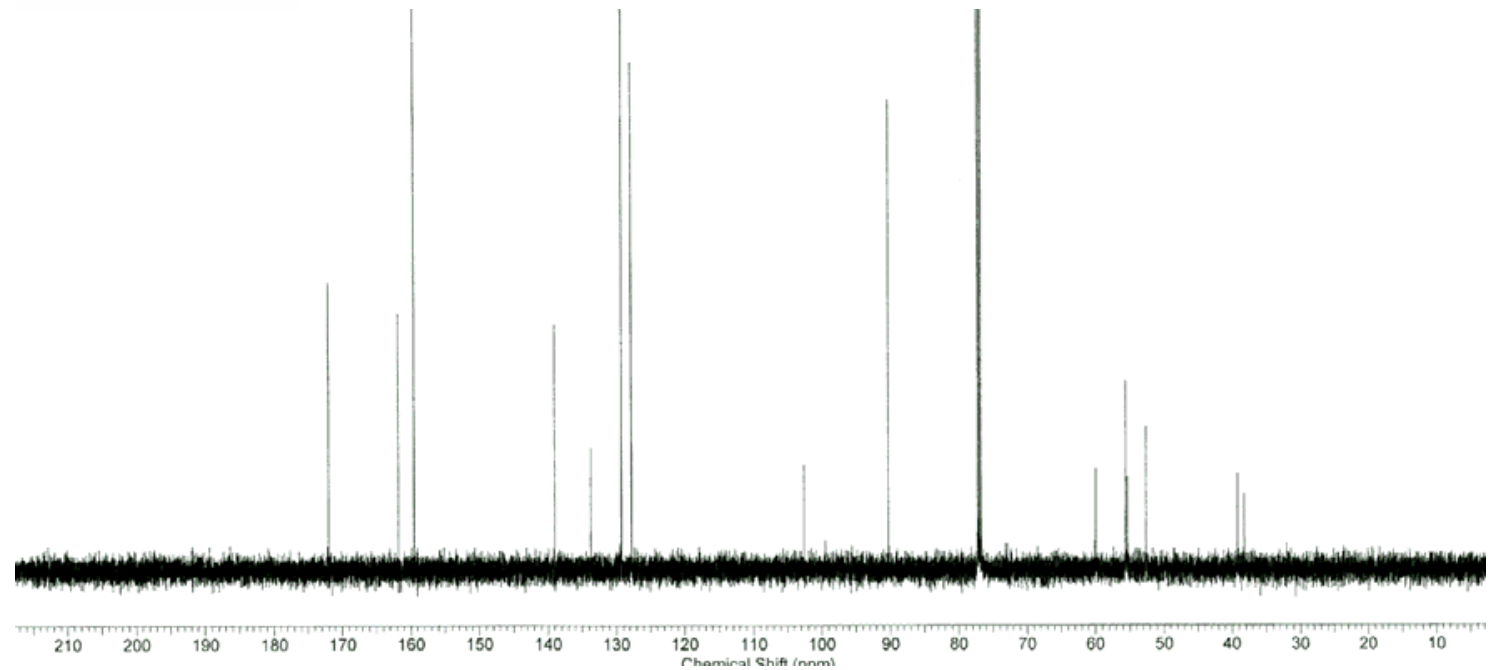


$300 \mathrm{MHz}\left(\mathrm{CDCl}_{3}\right)$
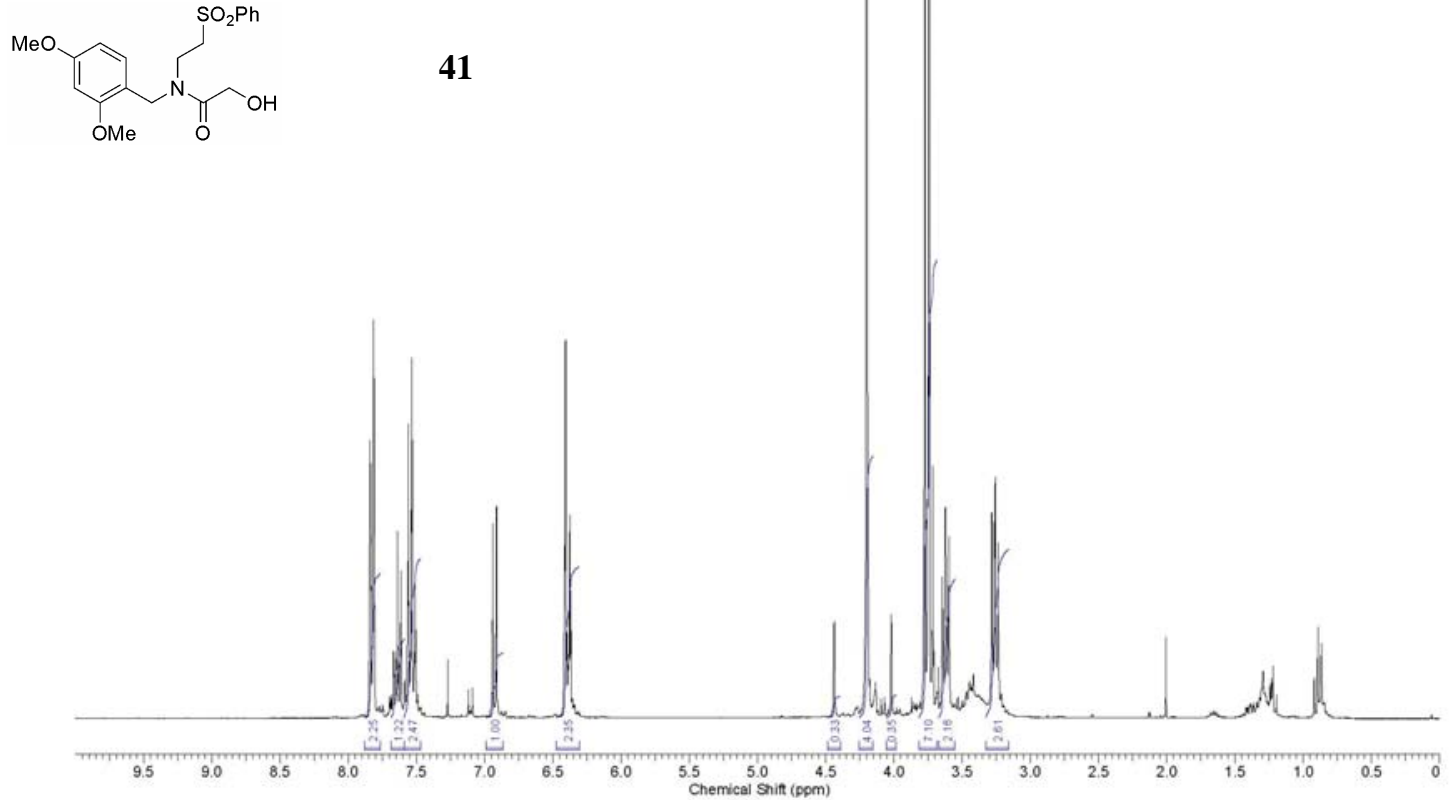

$75 \mathrm{MHz}\left(\mathrm{CDCl}_{3}\right)$

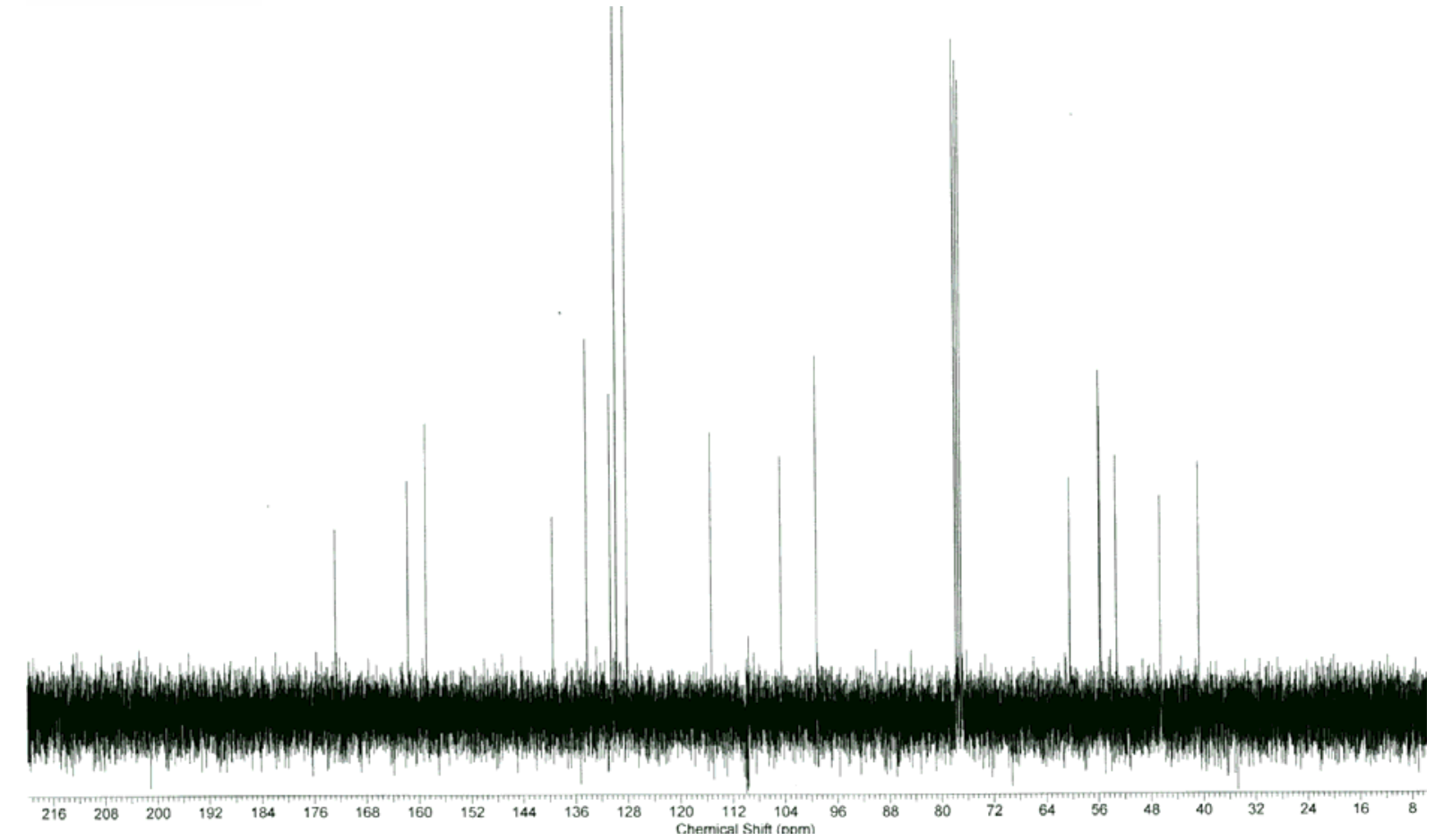


$500 \mathrm{MHz}\left(\mathrm{CDCl}_{3}\right)$
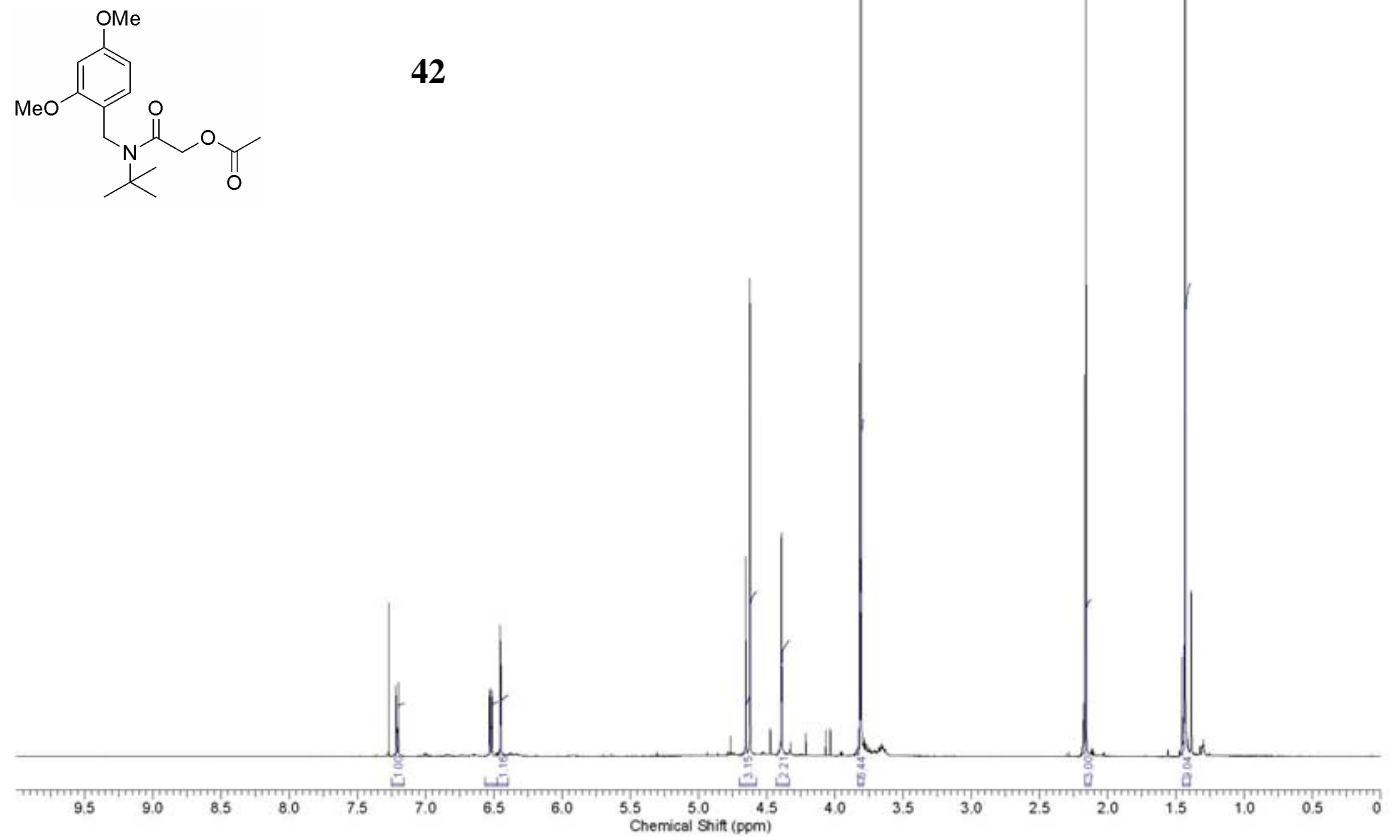

$125 \mathrm{MHz}\left(\mathrm{CDCl}_{3}\right)$

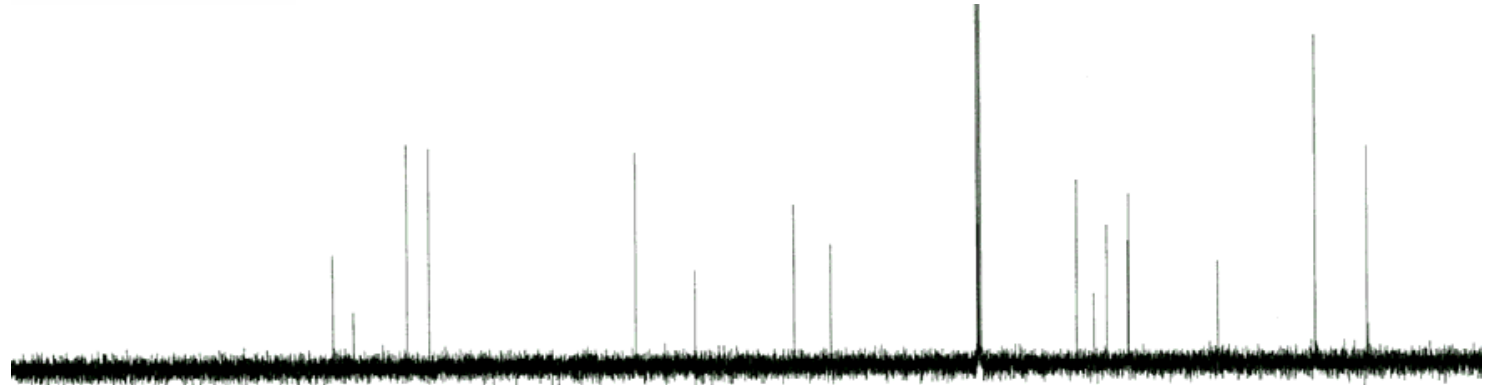

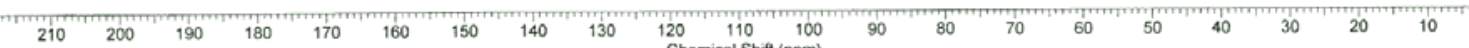


$500 \mathrm{MHz}\left(\mathrm{CDCl}_{3}\right)$
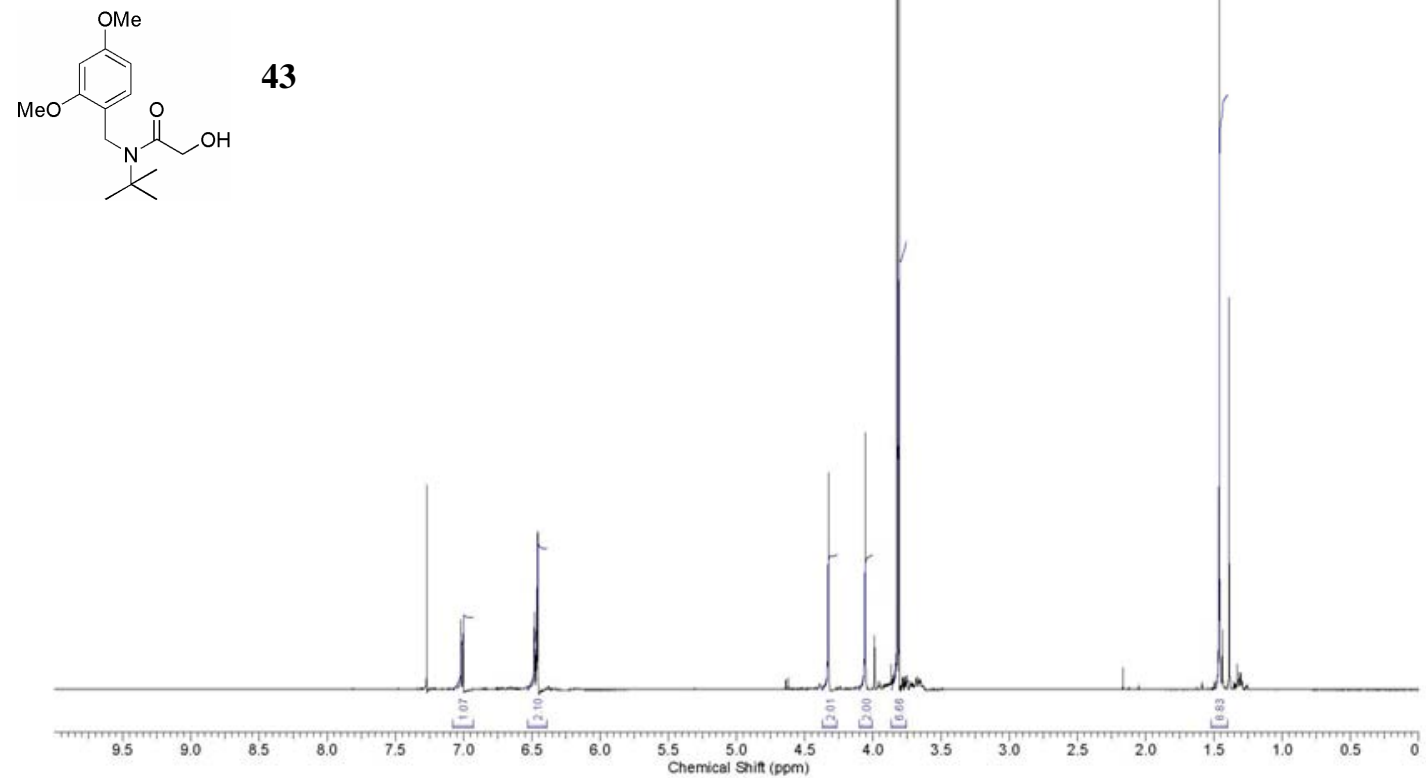

$125 \mathrm{MHz}\left(\mathrm{CDCl}_{3}\right)$

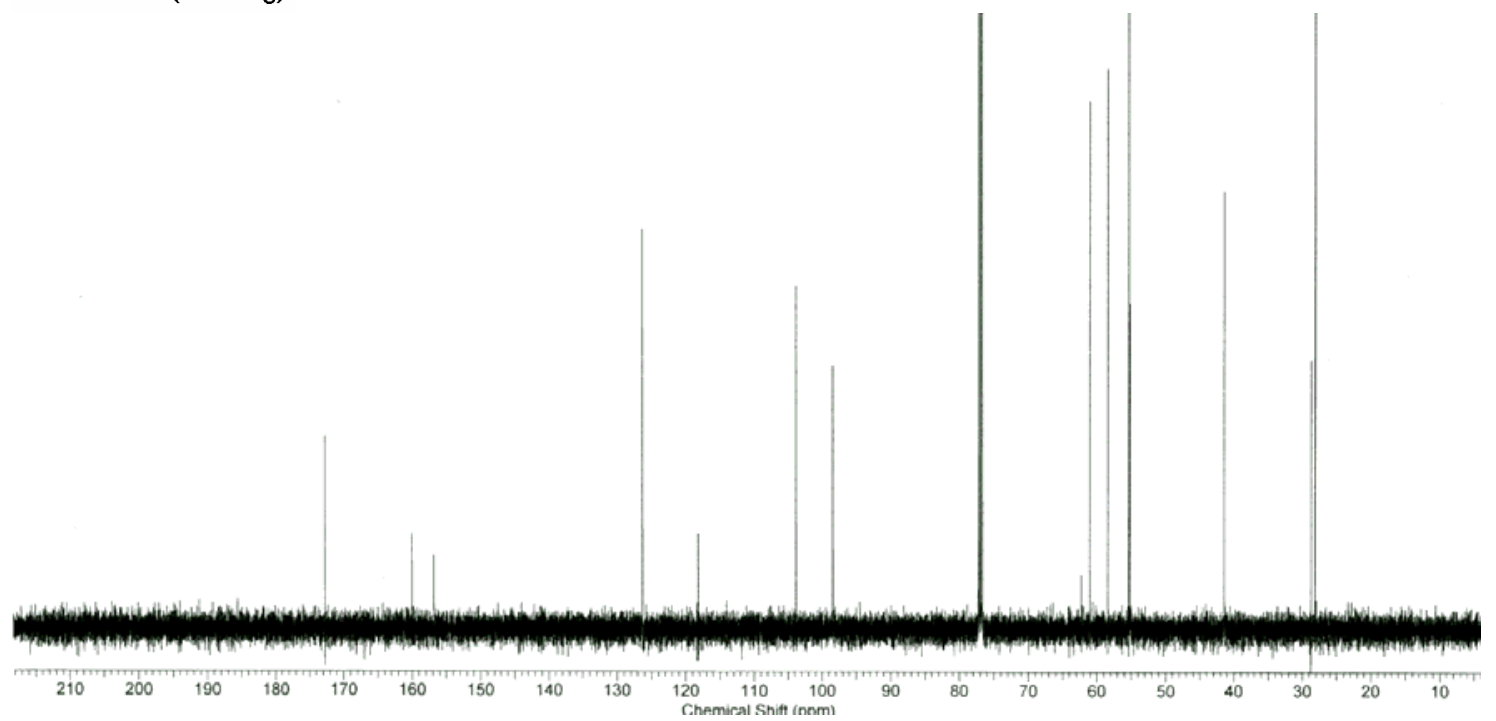


$300 \mathrm{MHz}\left(\mathrm{CDCl}_{3}\right)$

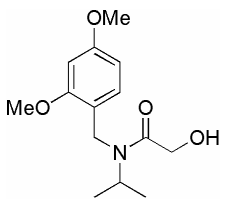

44

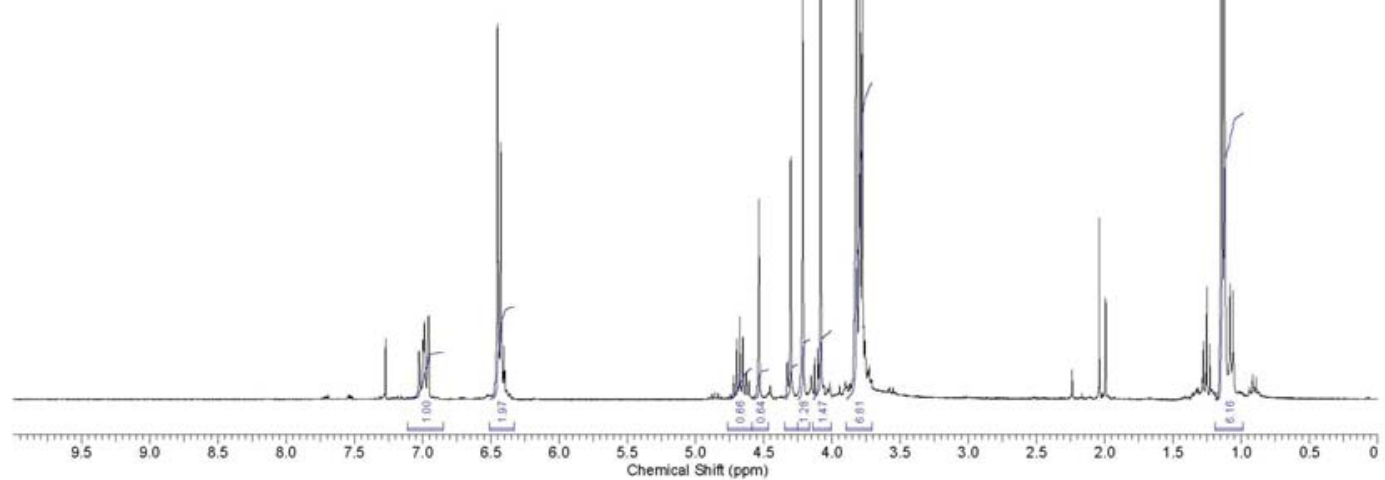

$75 \mathrm{MHz}\left(\mathrm{CDCl}_{3}\right)$
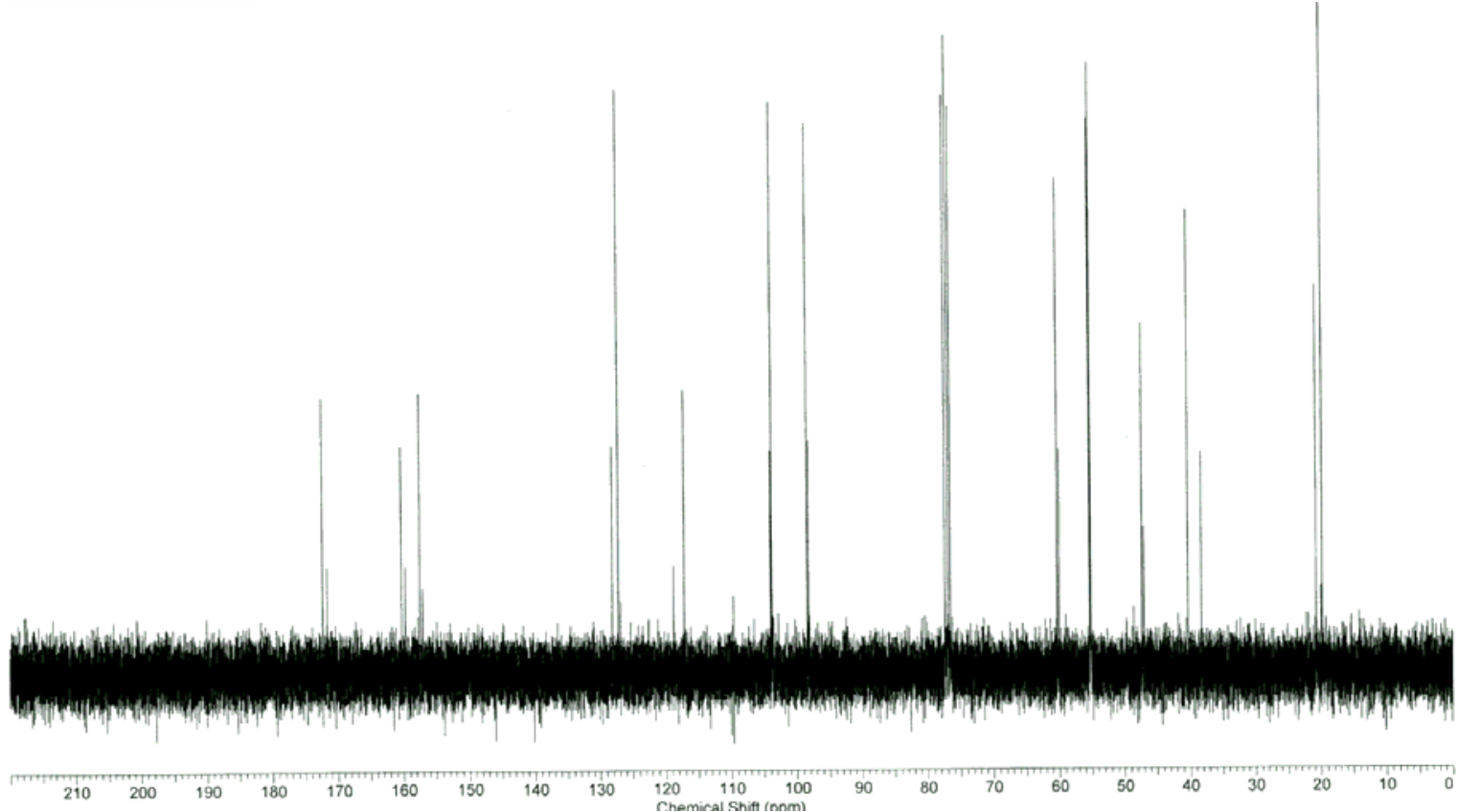


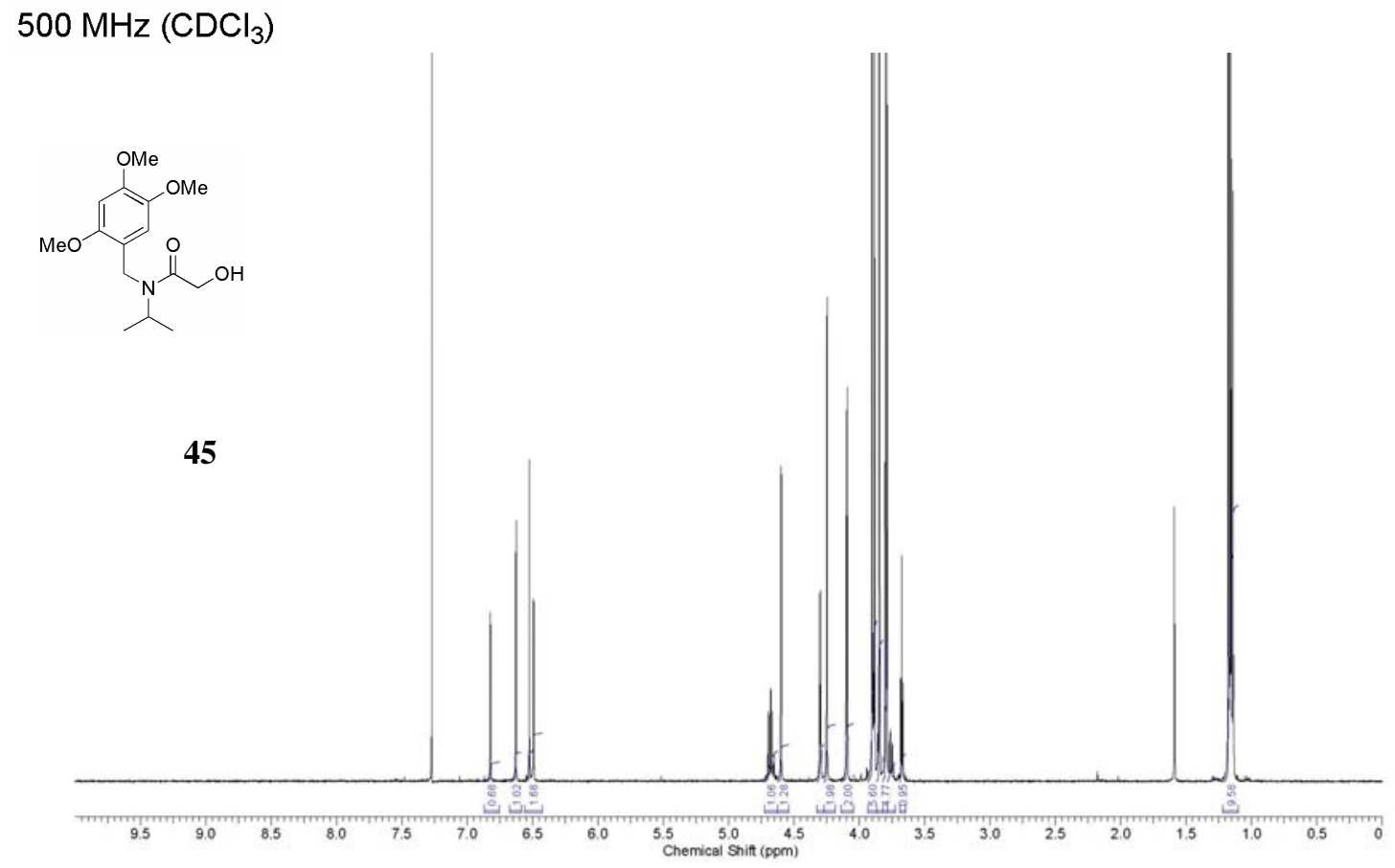

$125 \mathrm{MHz}\left(\mathrm{CDCl}_{3}\right)$

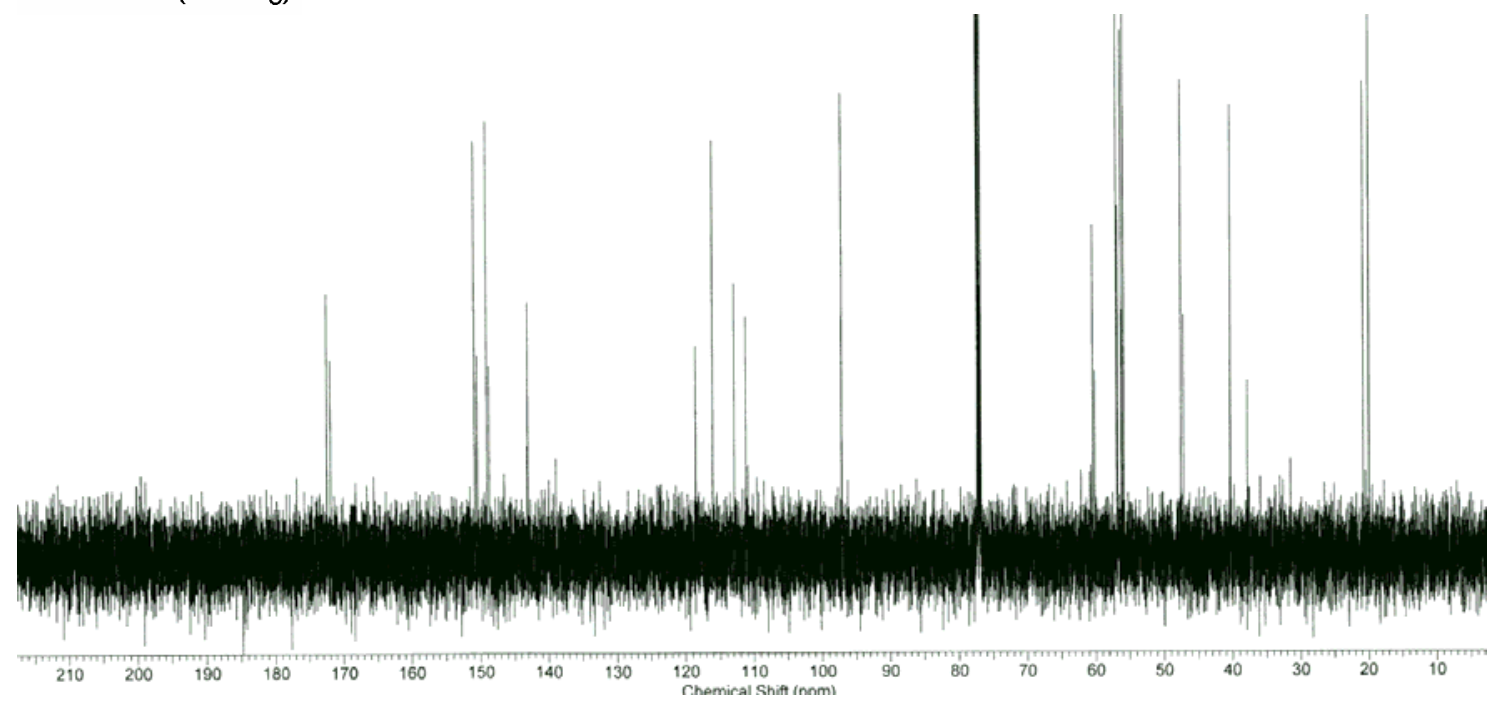


$500 \mathrm{MHz}\left(\mathrm{CDCl}_{3}\right)$
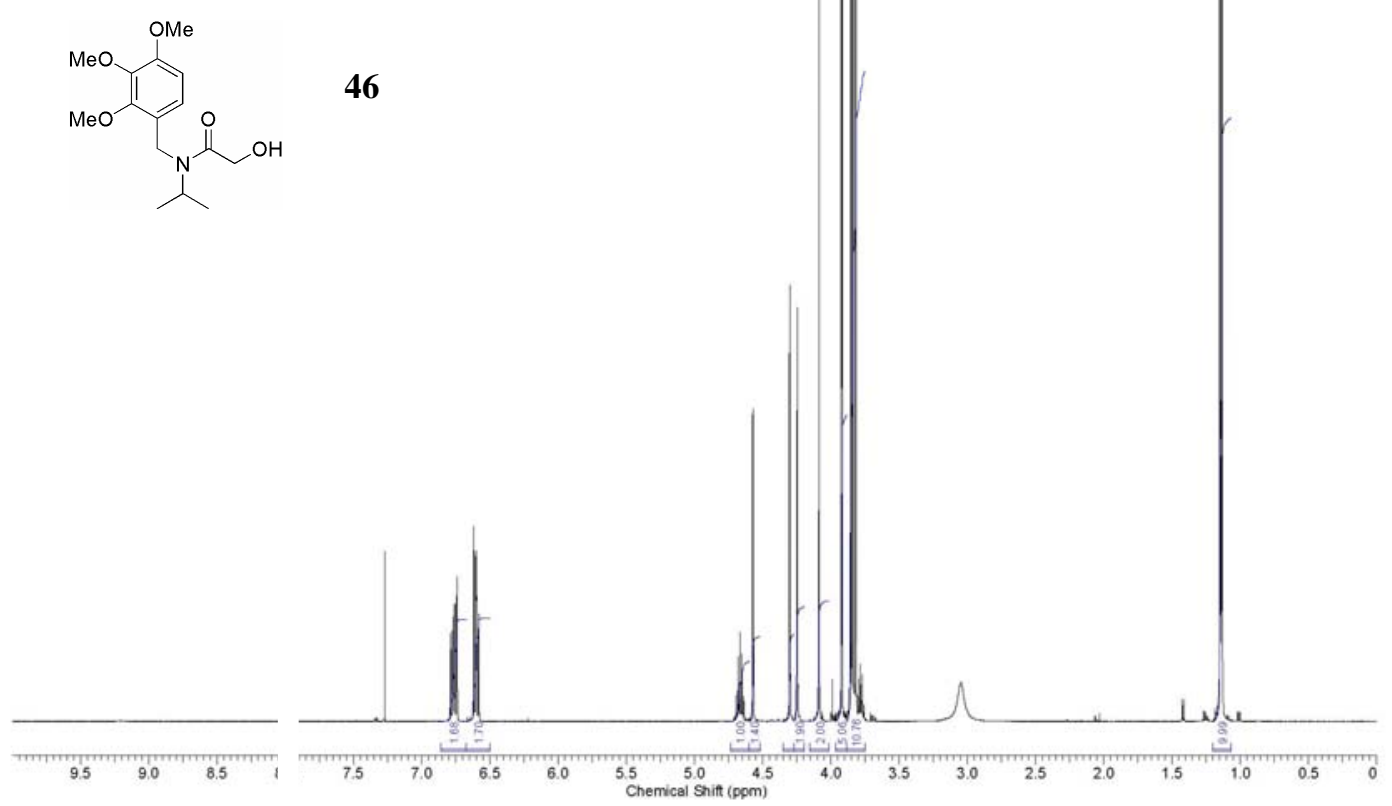

$125 \mathrm{MHz}\left(\mathrm{CDCl}_{3}\right)$

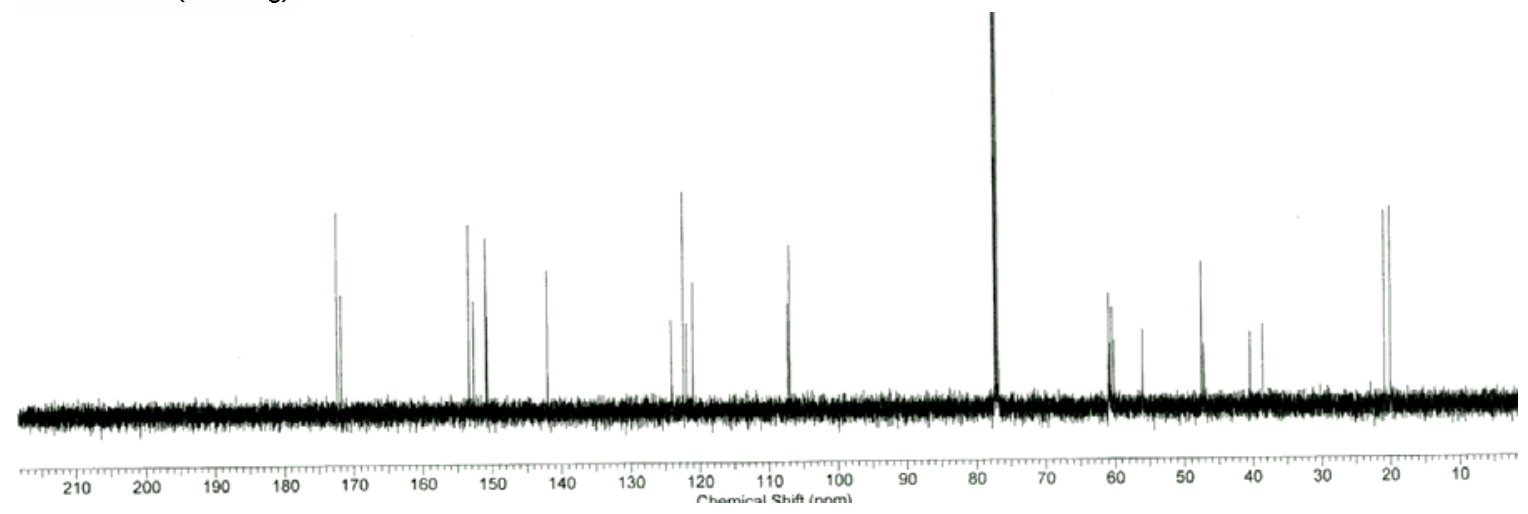




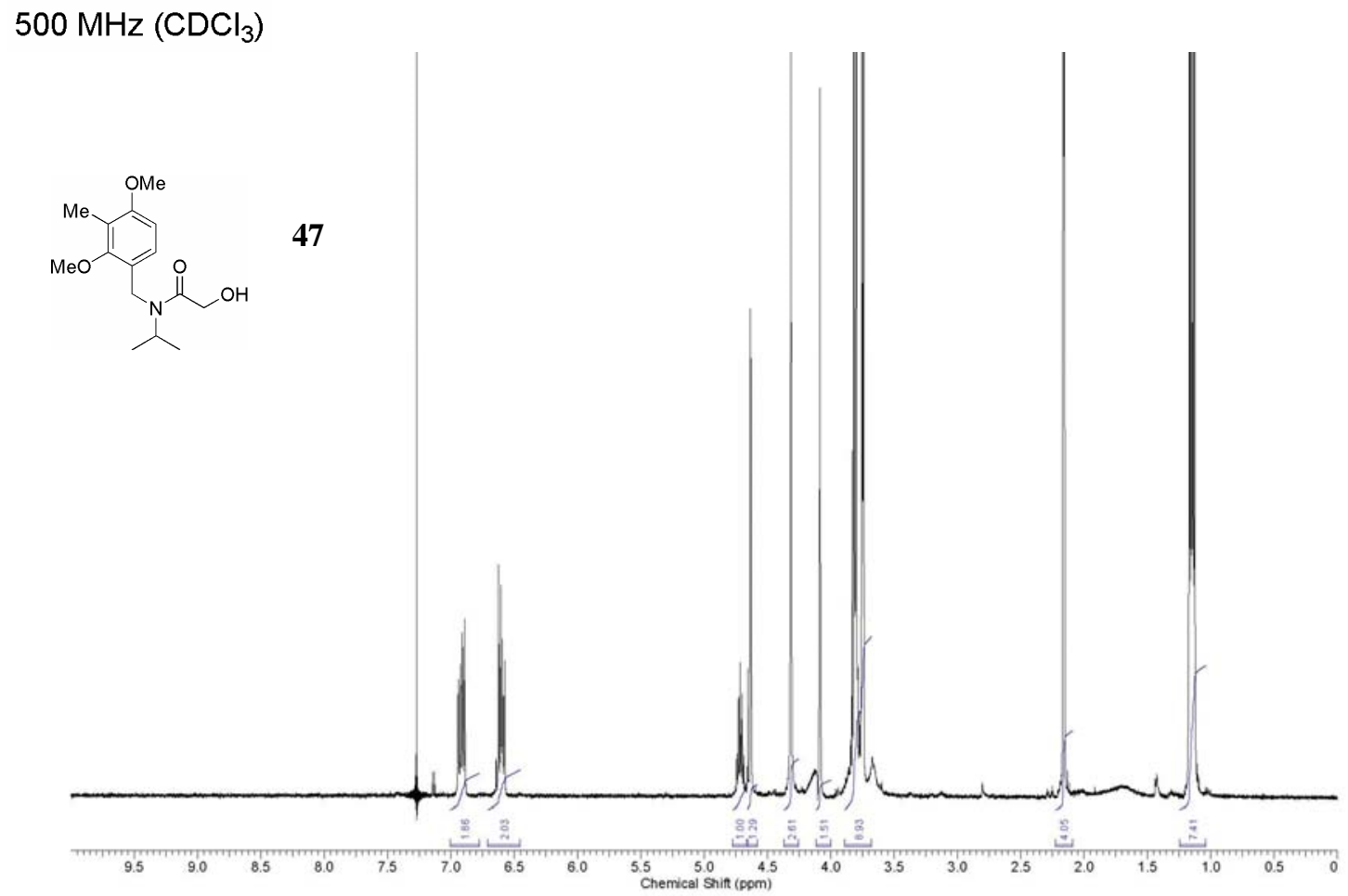

$125 \mathrm{MHz}\left(\mathrm{CDCl}_{3}\right)$

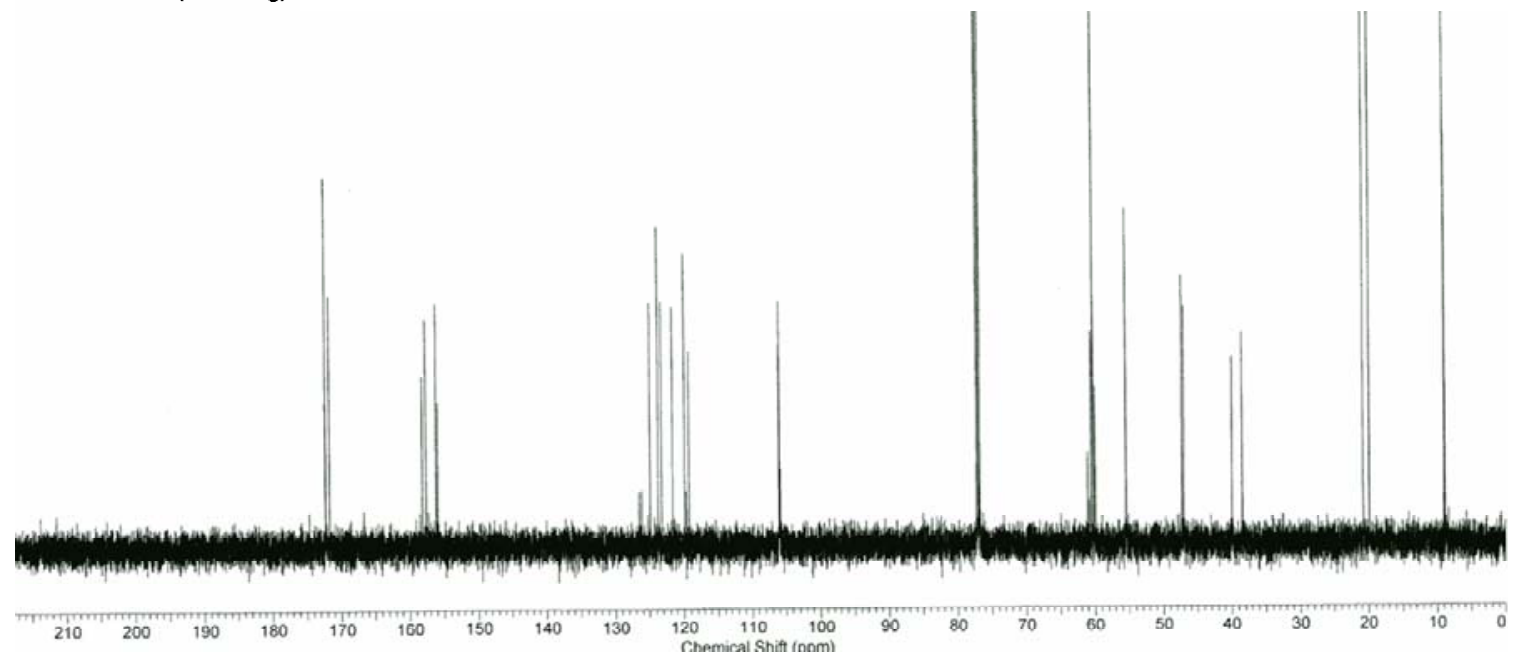


$500 \mathrm{MHz}\left(\mathrm{CDCl}_{3}\right)$<smiles>COc1cc(O)c(CN(C(=O)CO)C(C)C)c(F)c1</smiles>

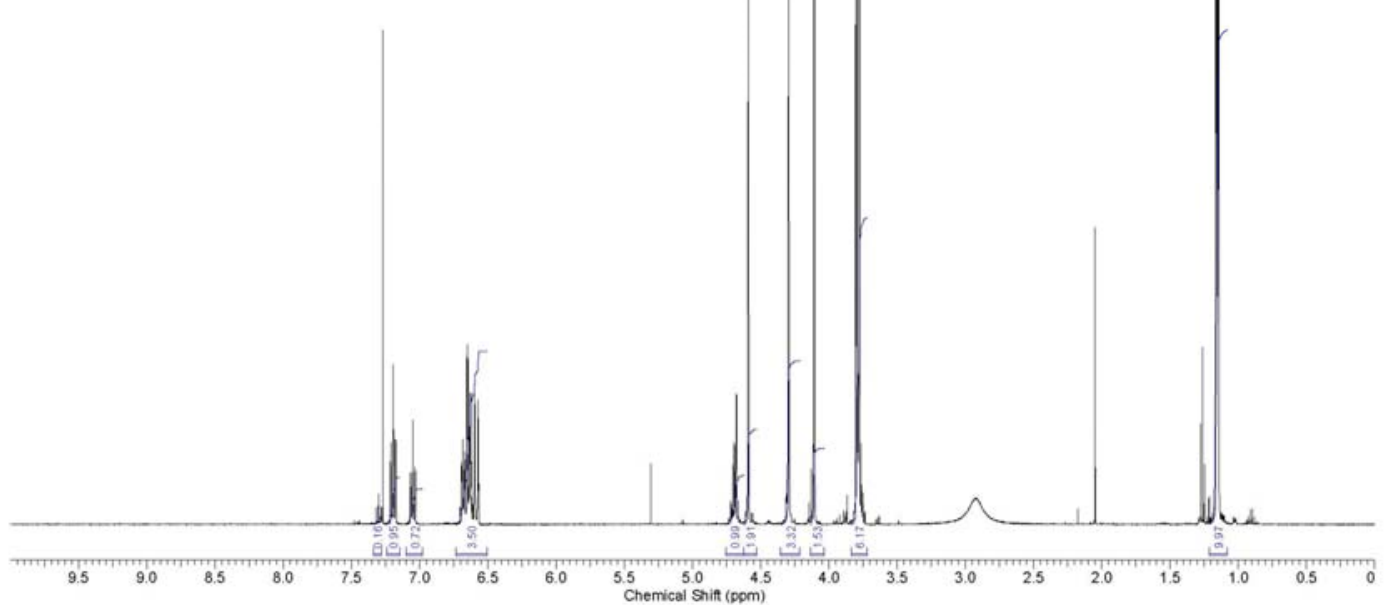

$125 \mathrm{MHz}\left(\mathrm{CDCl}_{3}\right)$

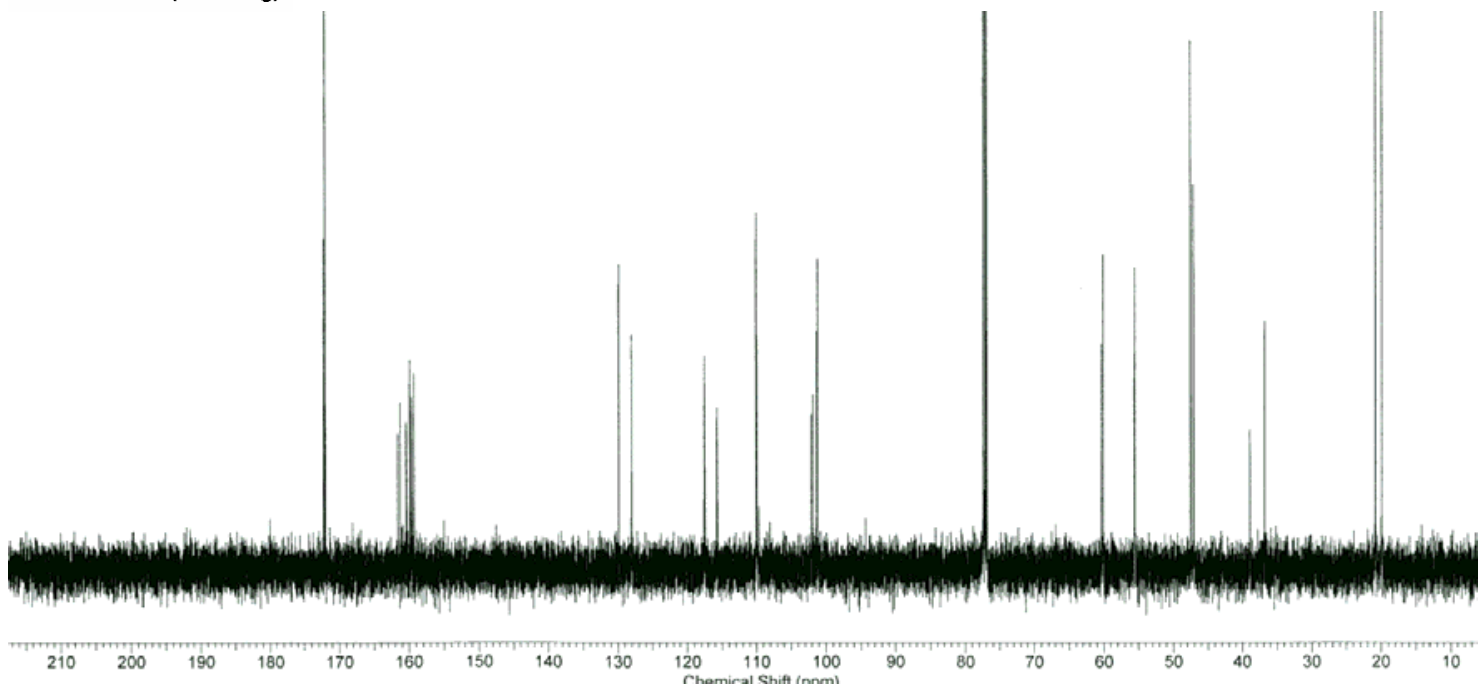


$500 \mathrm{MHz}\left(\mathrm{CDCl}_{3}\right)$

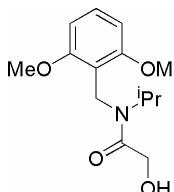

49

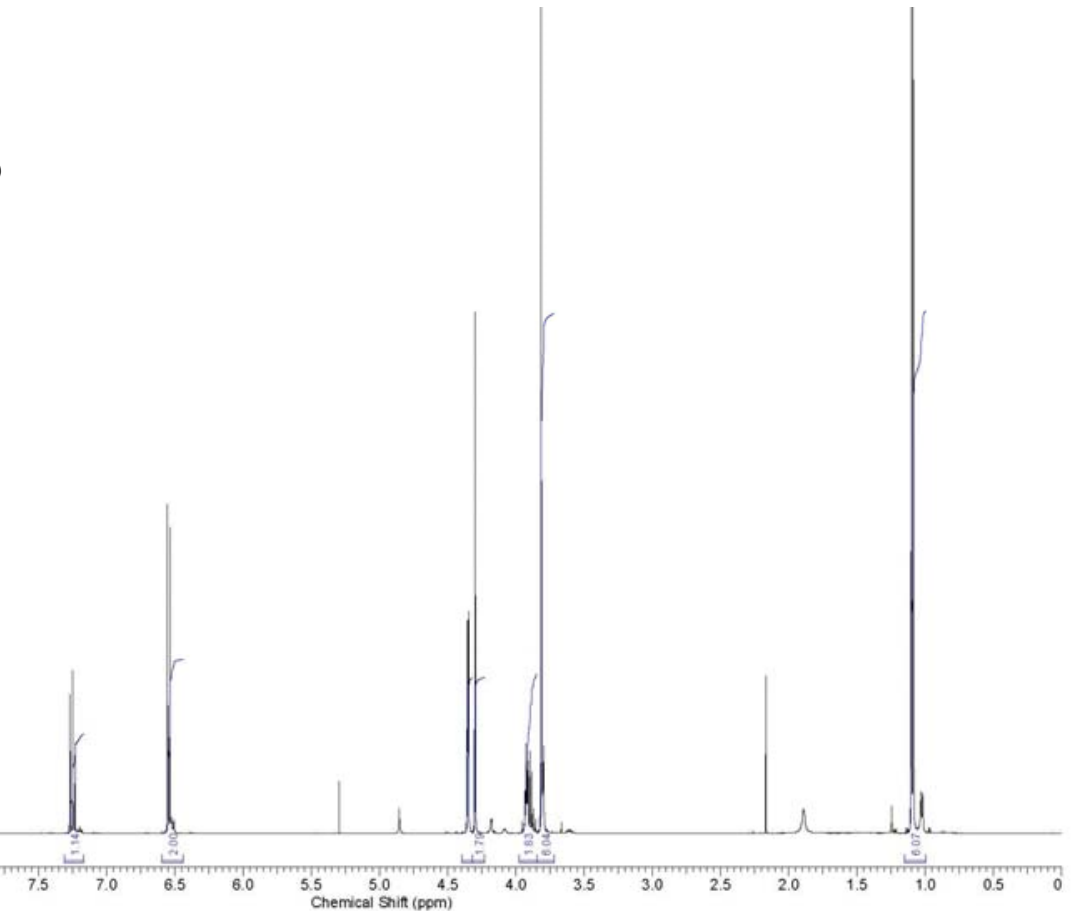

$125 \mathrm{MHz}\left(\mathrm{CDCl}_{3}\right)$

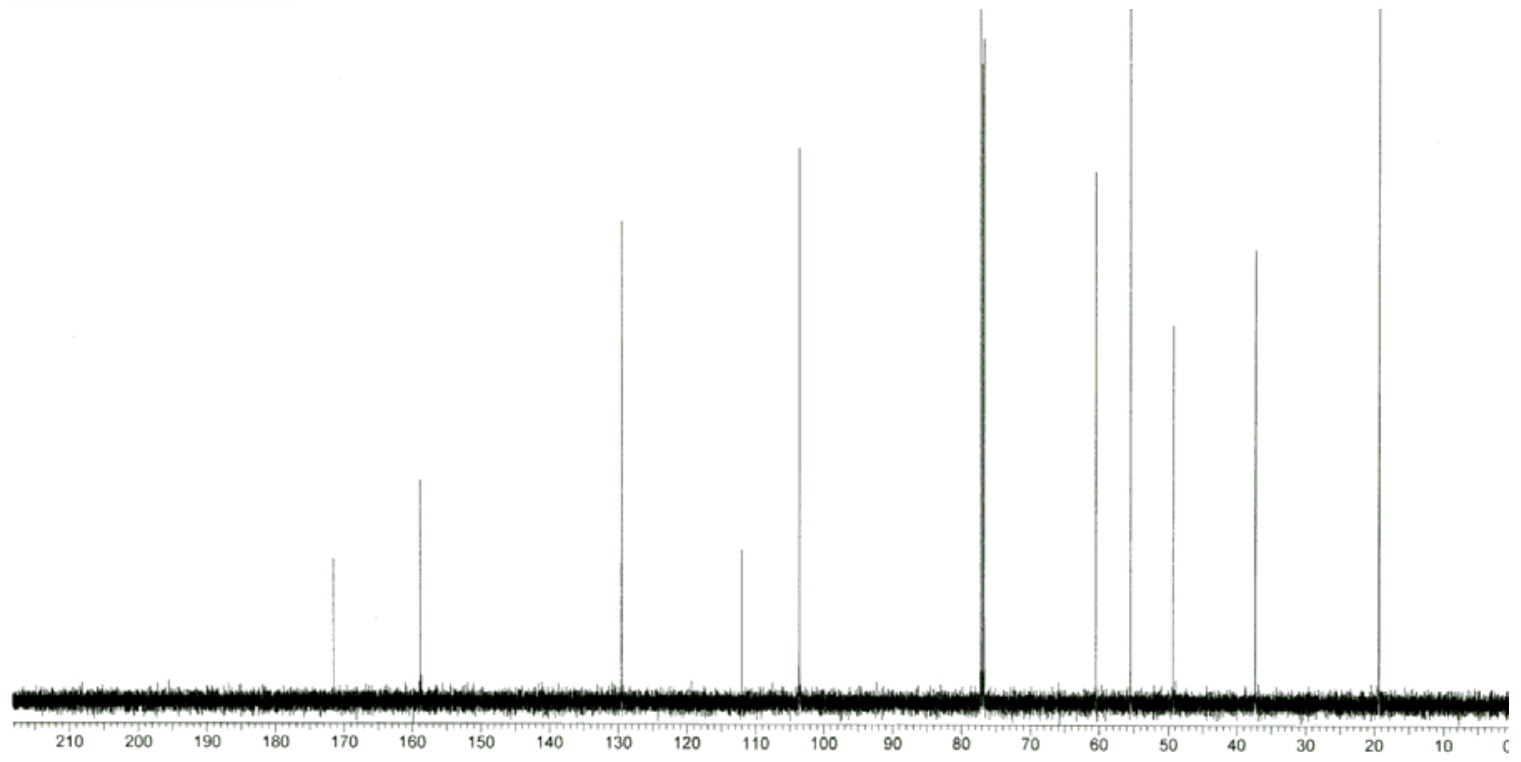


NMR spectra: cyclisations

$400 \mathrm{MHz}\left(\mathrm{CDCl}_{3}\right)$

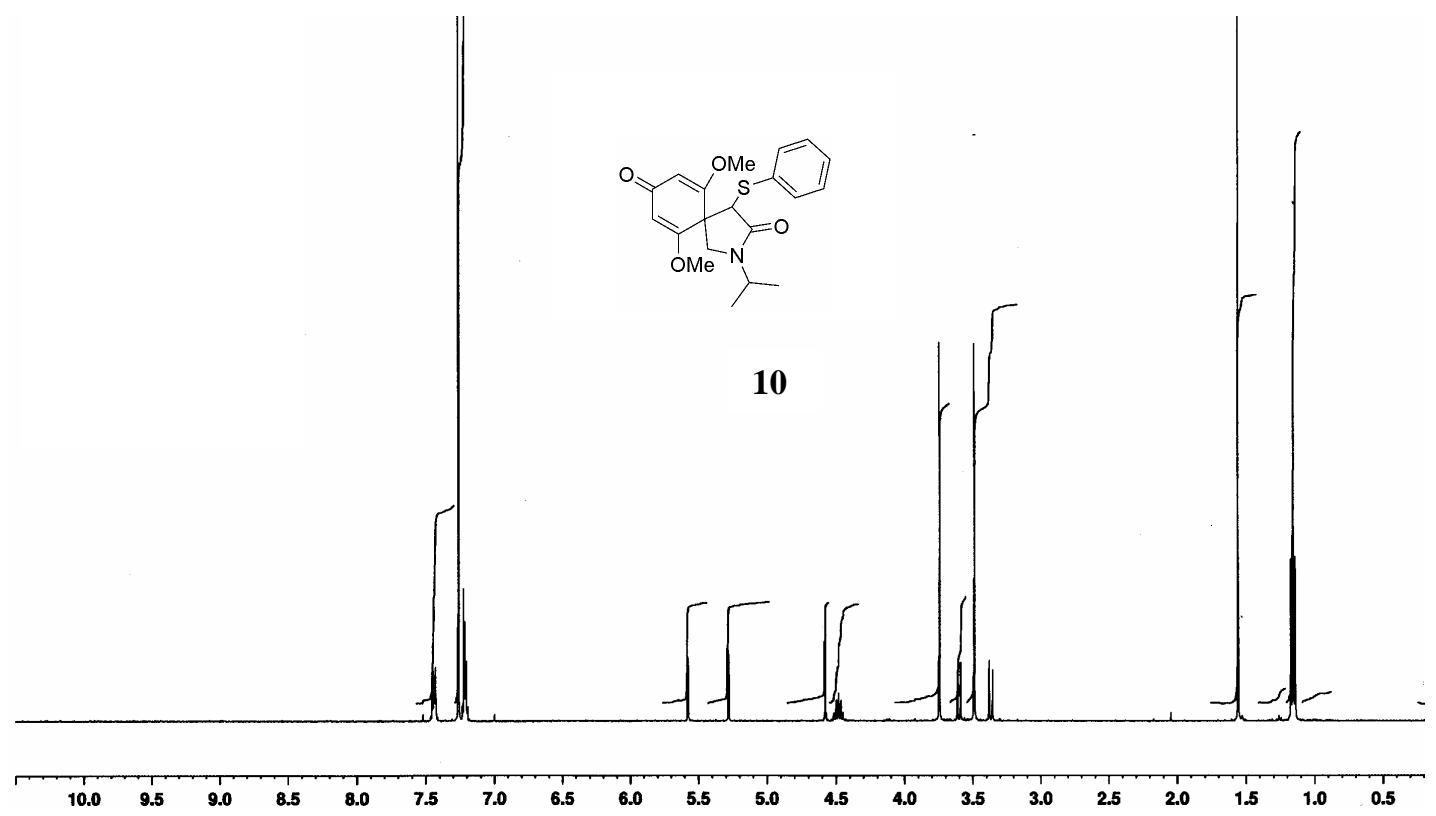

$100 \mathrm{MHz}\left(\mathrm{CDCl}_{3}\right)$

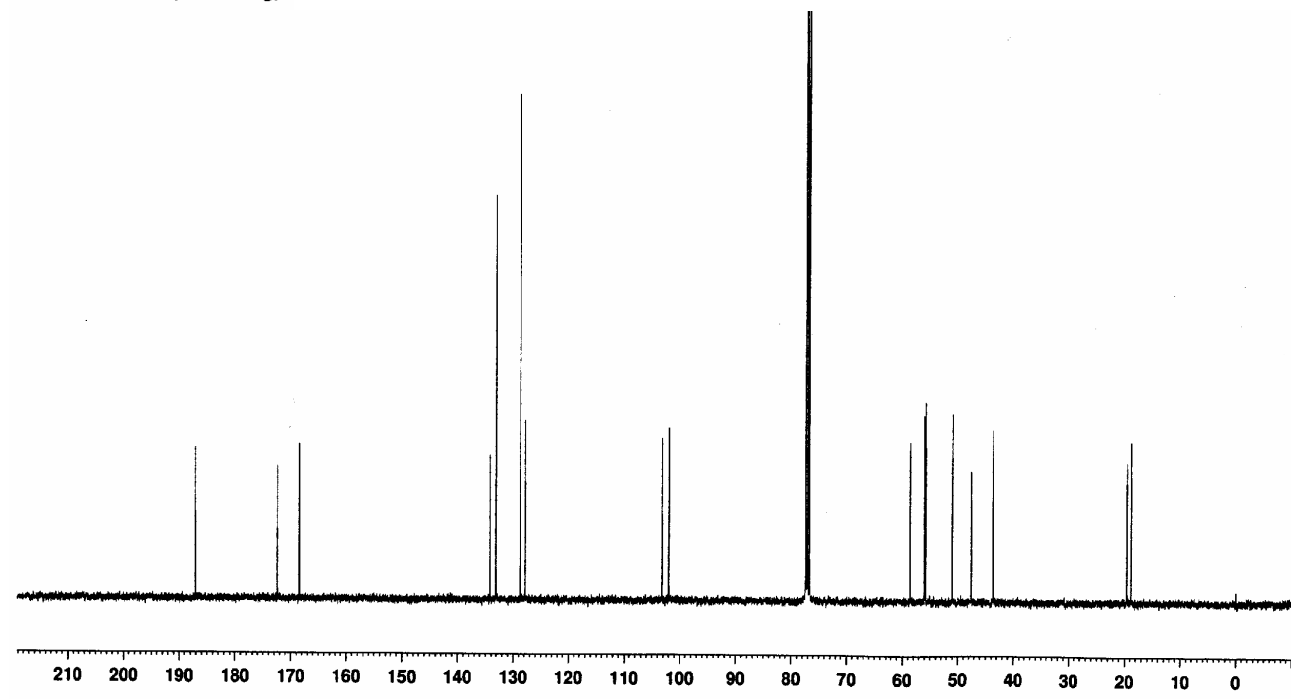


$500 \mathrm{MHz}\left(\mathrm{CDCl}_{3}\right)$

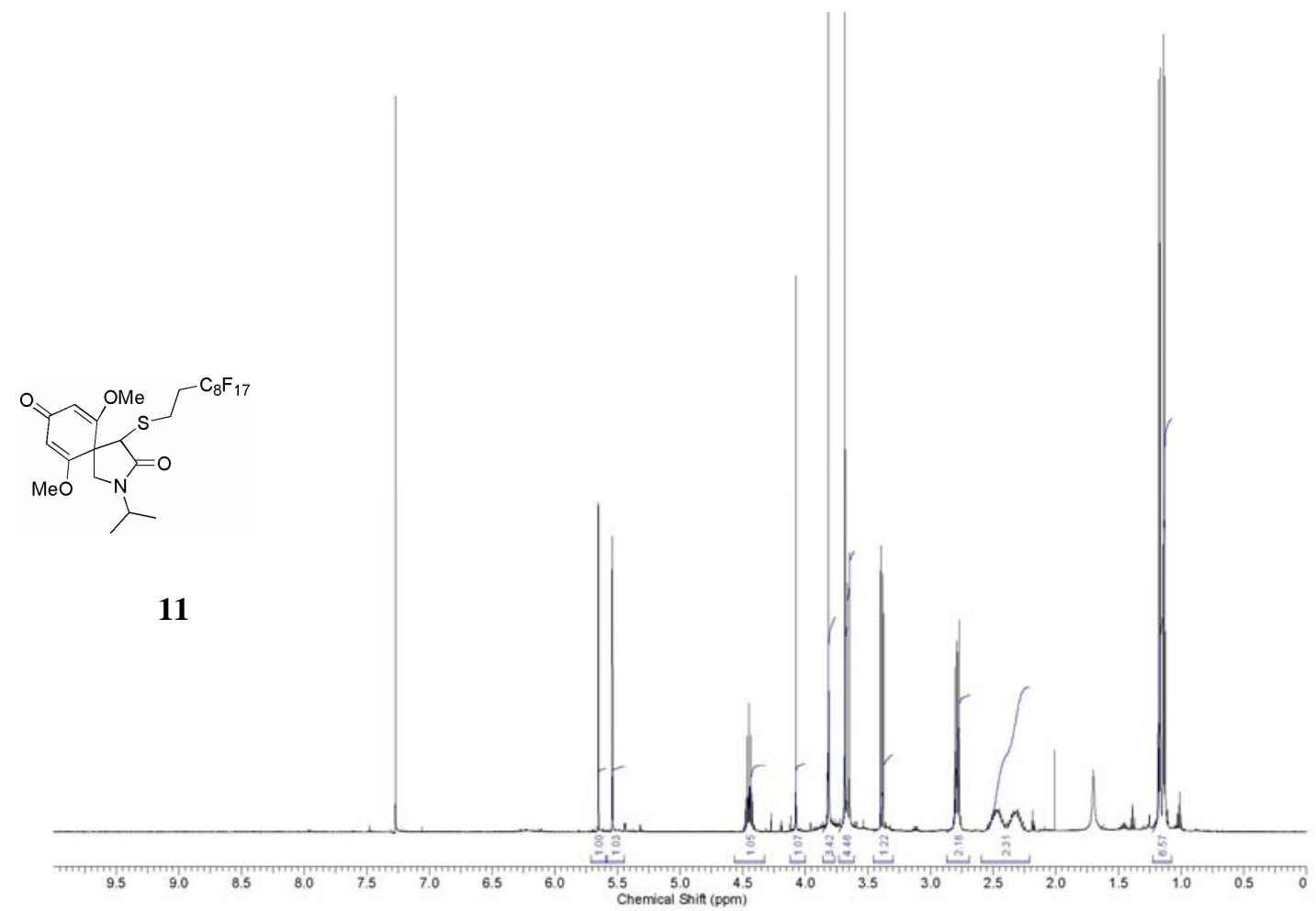

$75 \mathrm{MHz}\left(\mathrm{CDCl}_{3}\right)$

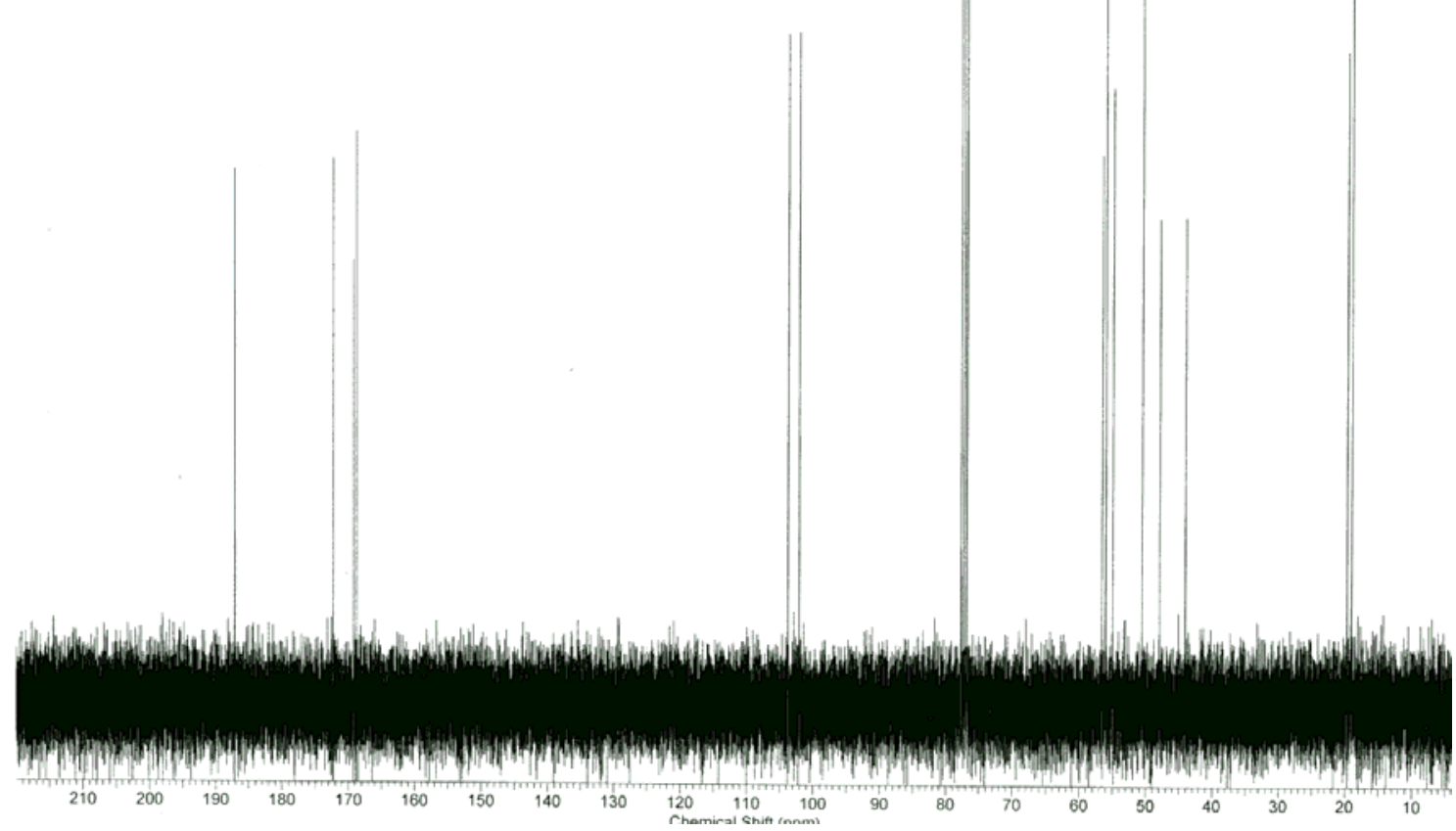


$500 \mathrm{MHz}\left(\mathrm{CDCl}_{3}\right)$
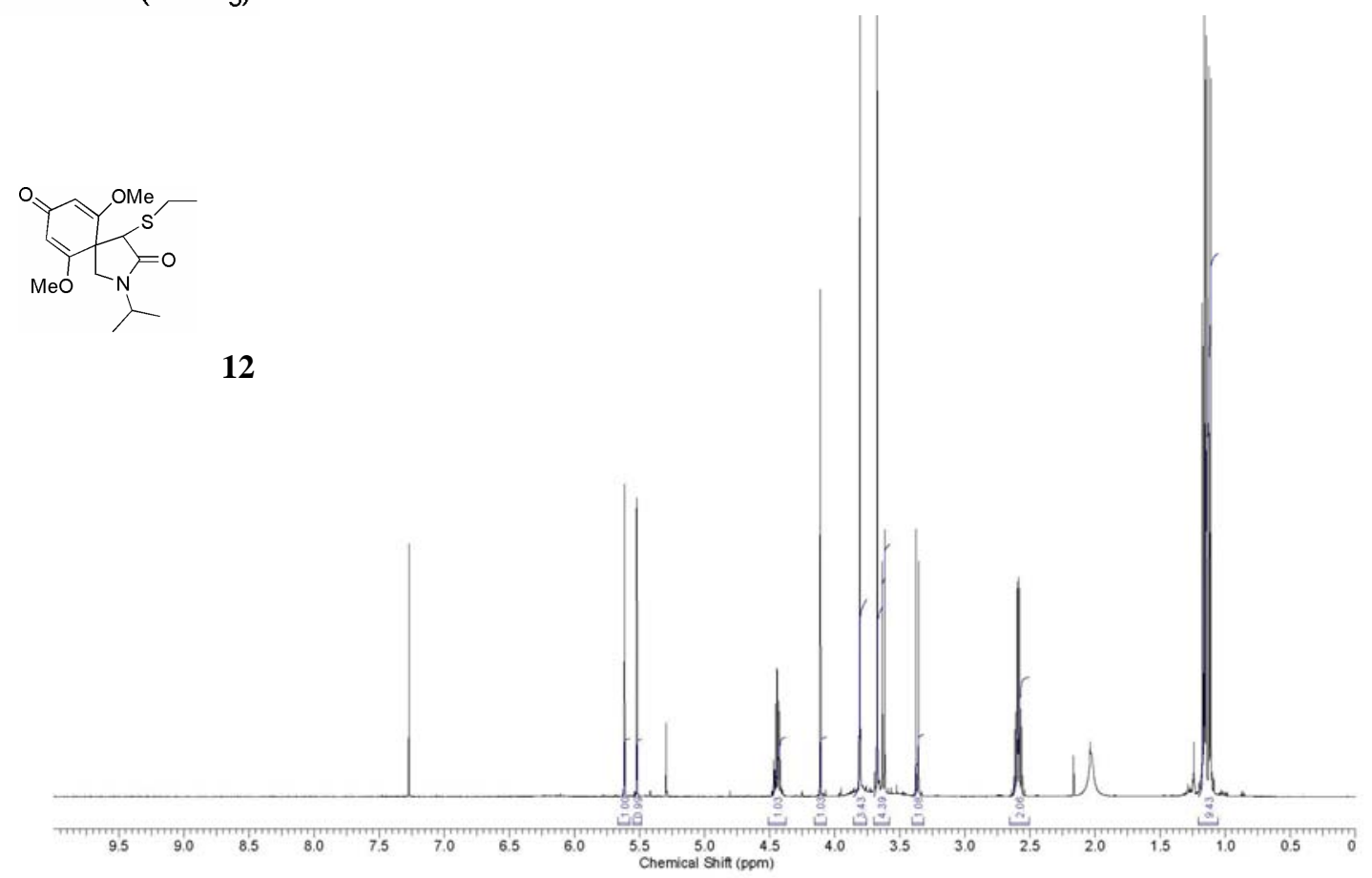

$125 \mathrm{MHz}\left(\mathrm{CDCl}_{3}\right)$

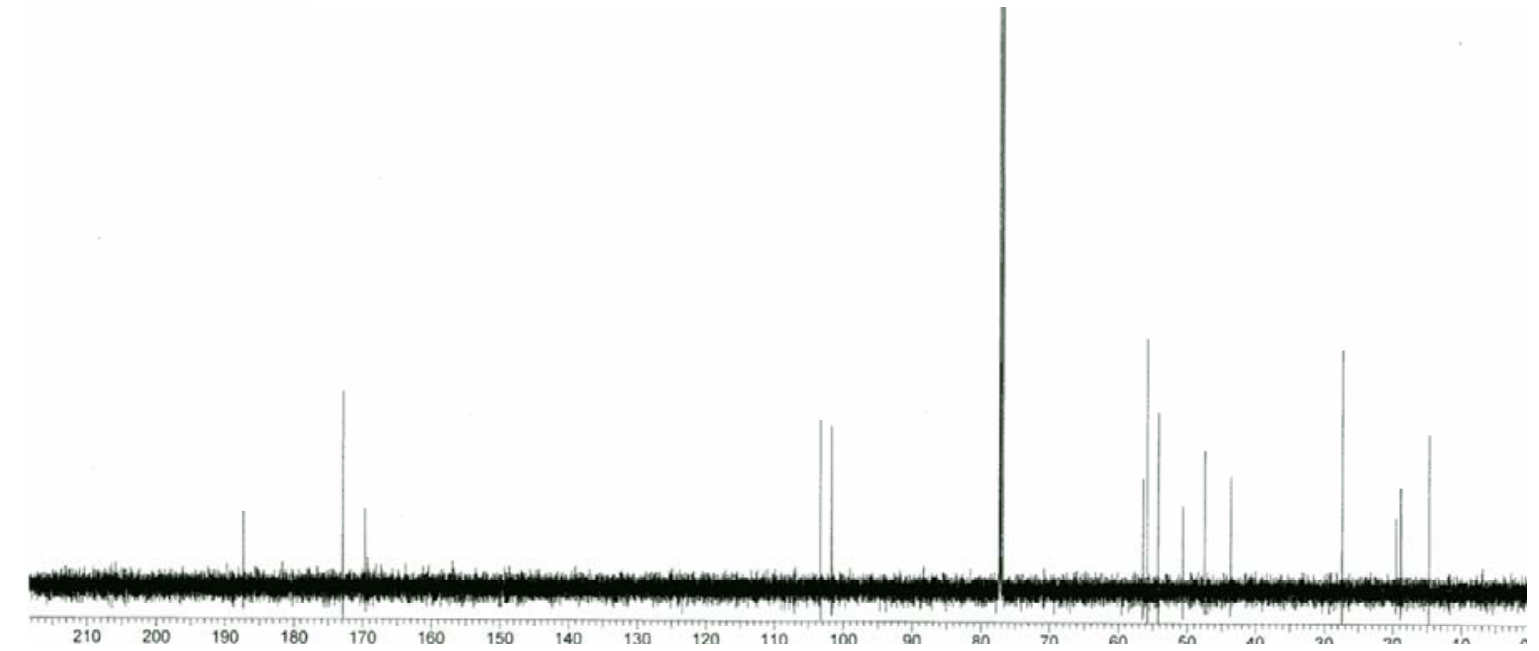




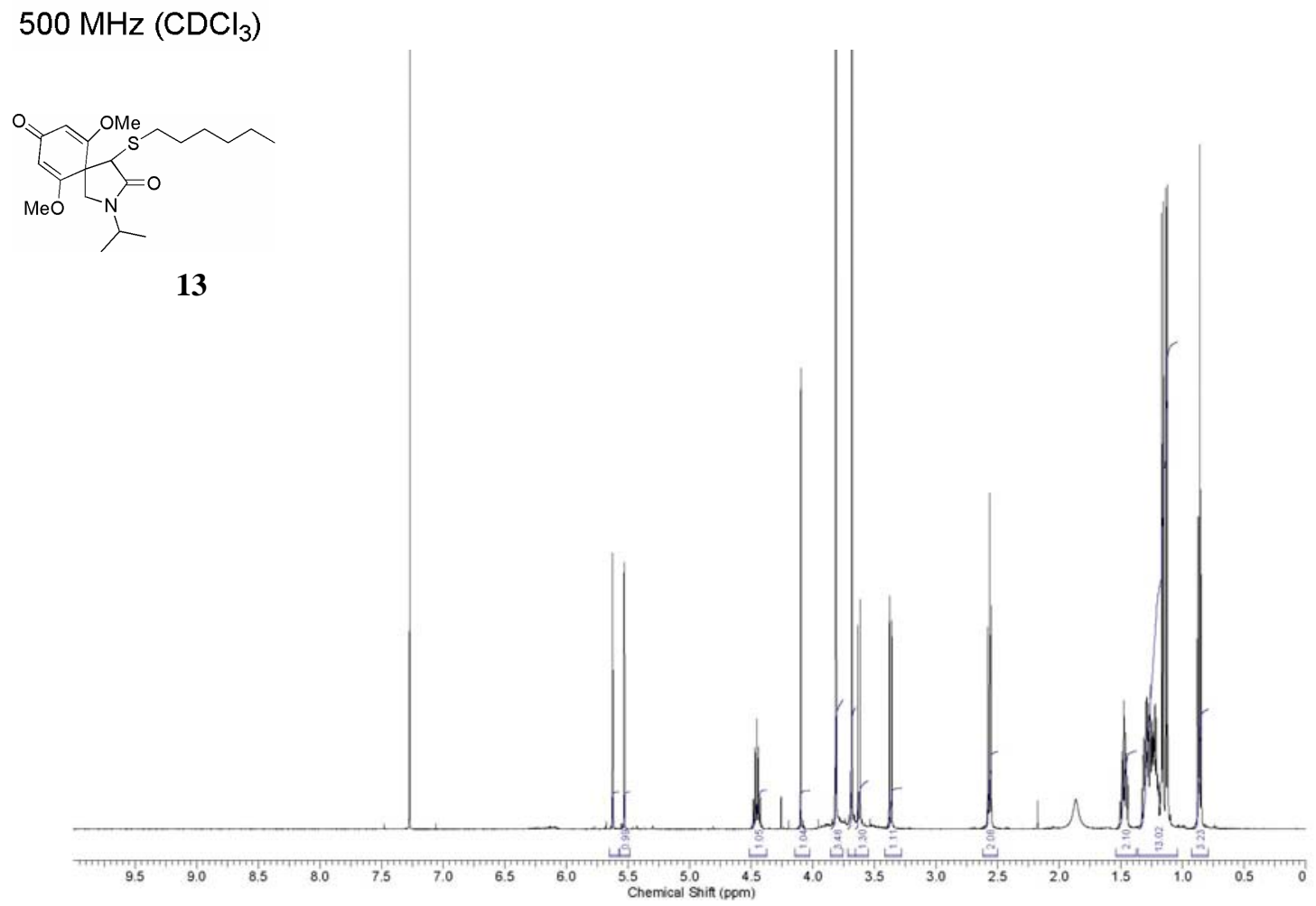

$125 \mathrm{MHz}\left(\mathrm{CDCl}_{3}\right)$

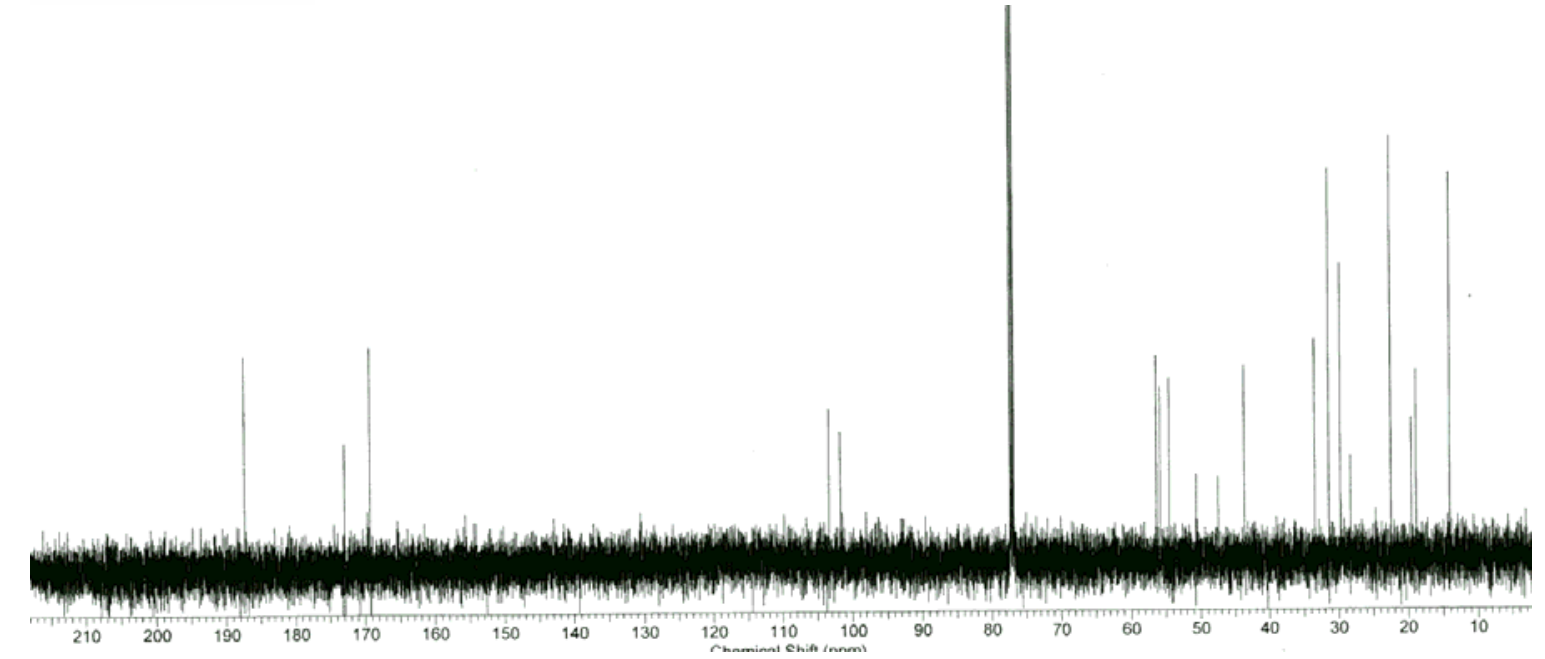


$500 \mathrm{MHz}\left(\mathrm{CDCl}_{3}\right)$

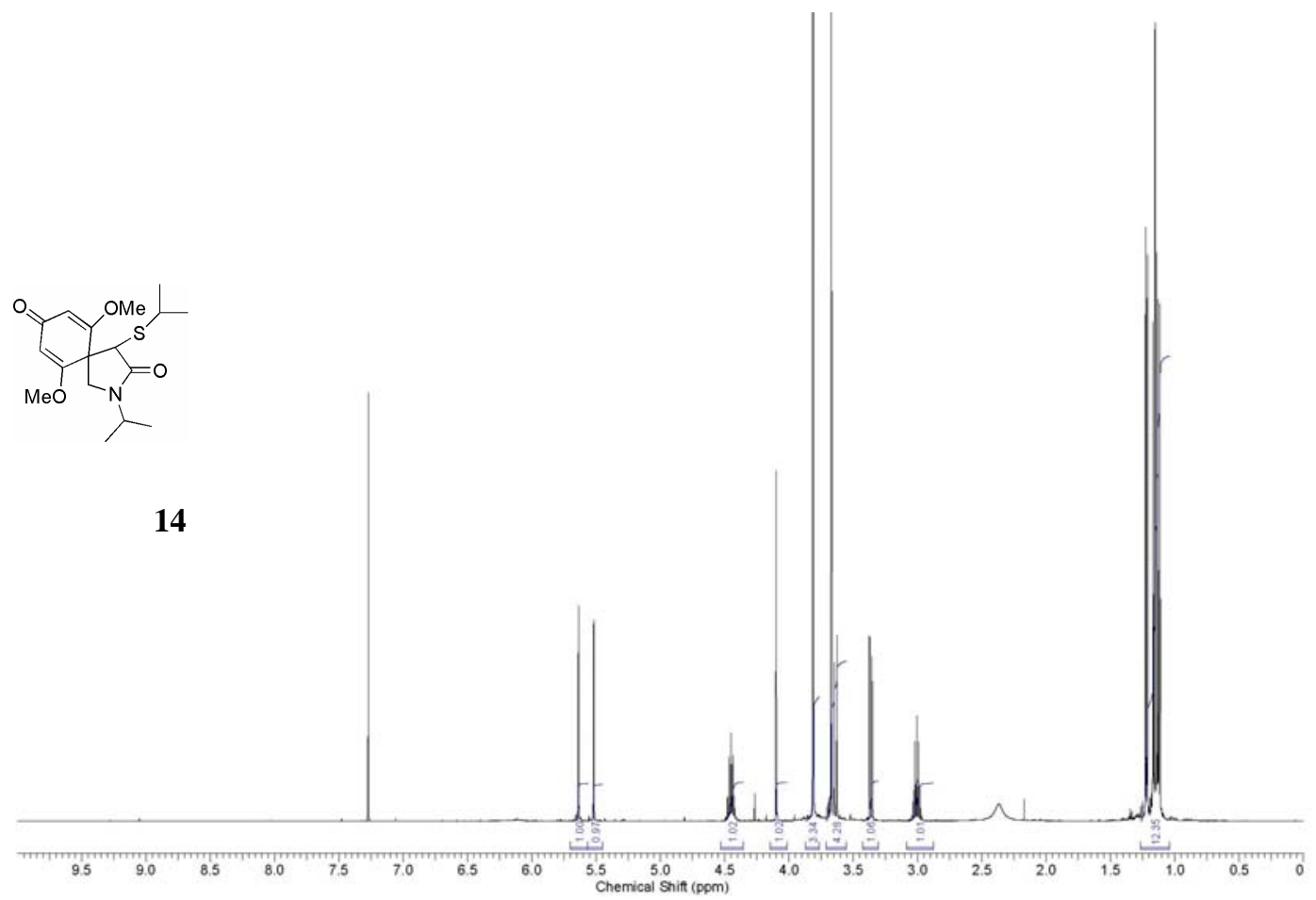

$125 \mathrm{MHz}\left(\mathrm{CDCl}_{3}\right)$

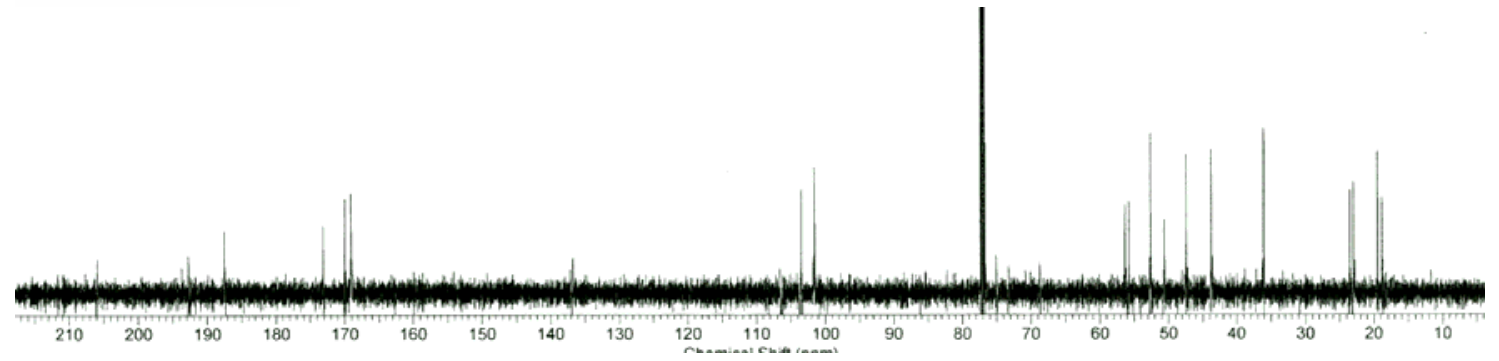


$500 \mathrm{MHz}\left(\mathrm{CDCl}_{3}\right)$

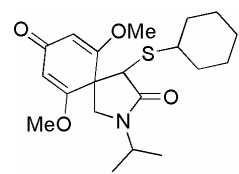

15

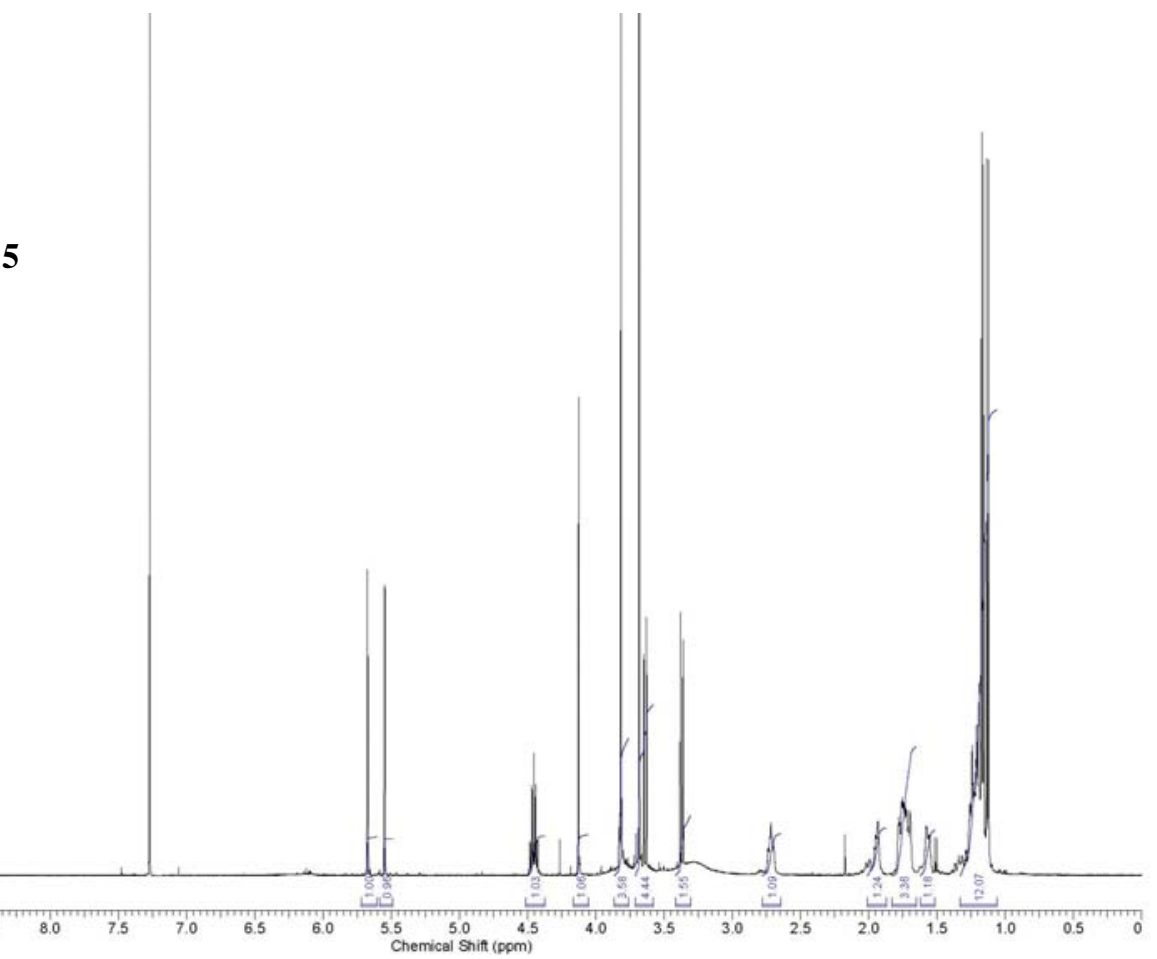

$125 \mathrm{MHz}\left(\mathrm{CDCl}_{3}\right)$

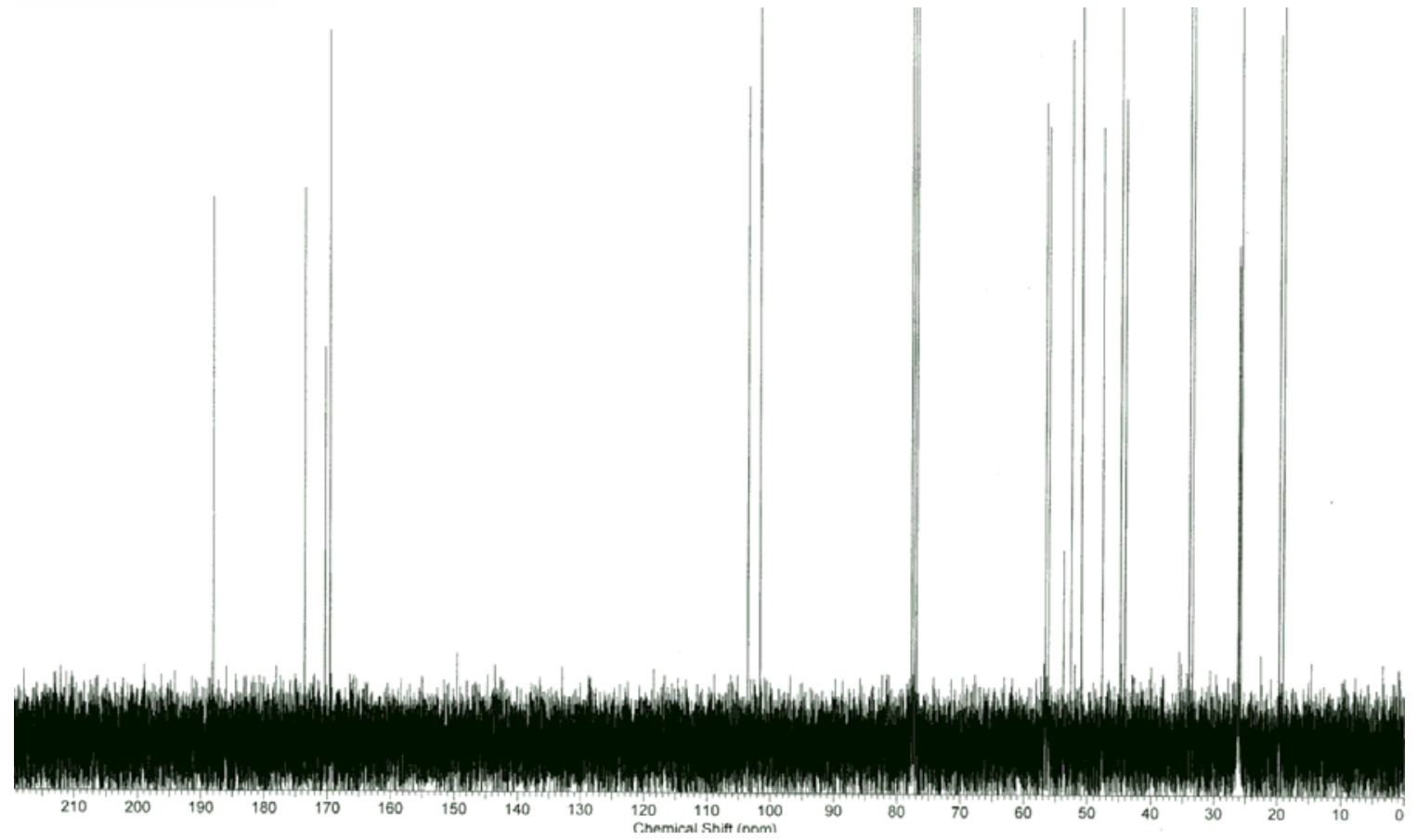


$500 \mathrm{MHz}\left(\mathrm{CDCl}_{3}\right)$

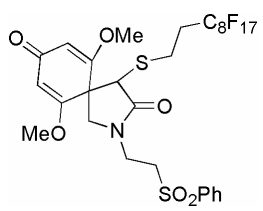

16

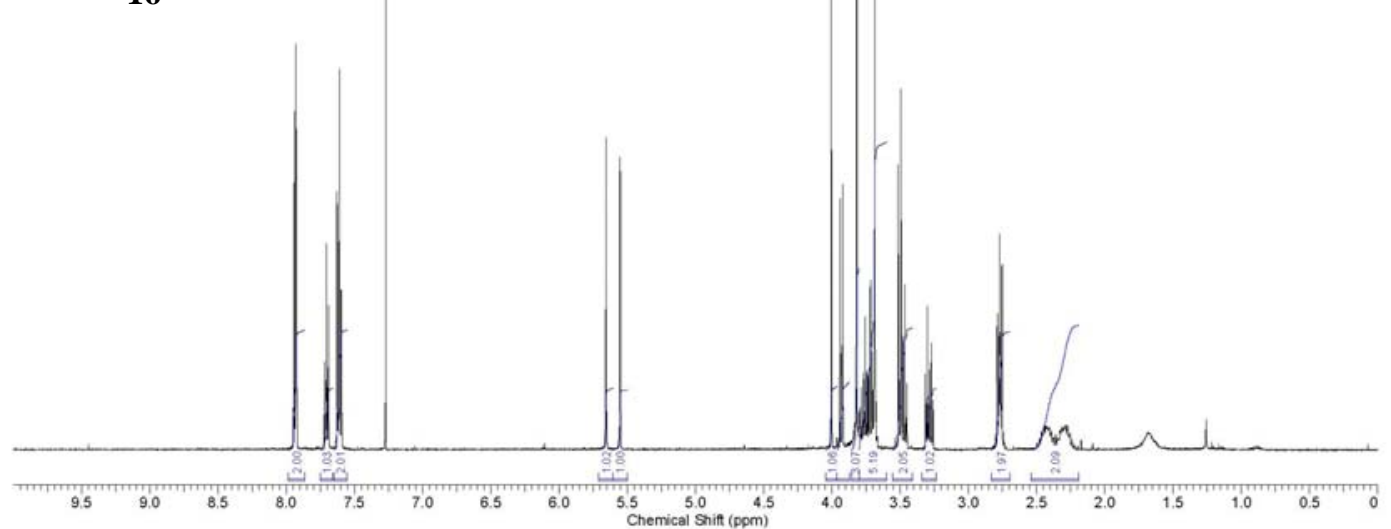

$75 \mathrm{MHz}\left(\mathrm{CDCl}_{3}\right)$

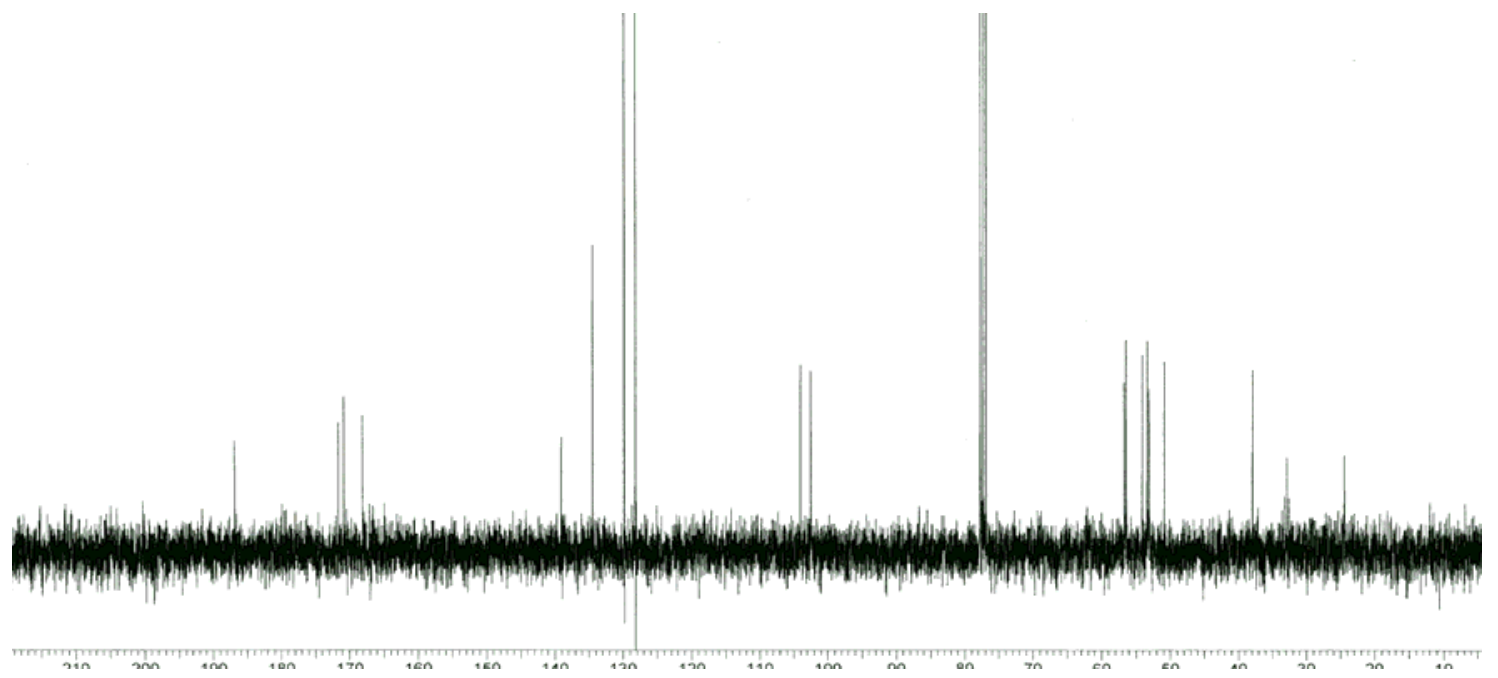


$500 \mathrm{MHz}\left(\mathrm{CDCl}_{3}\right)$

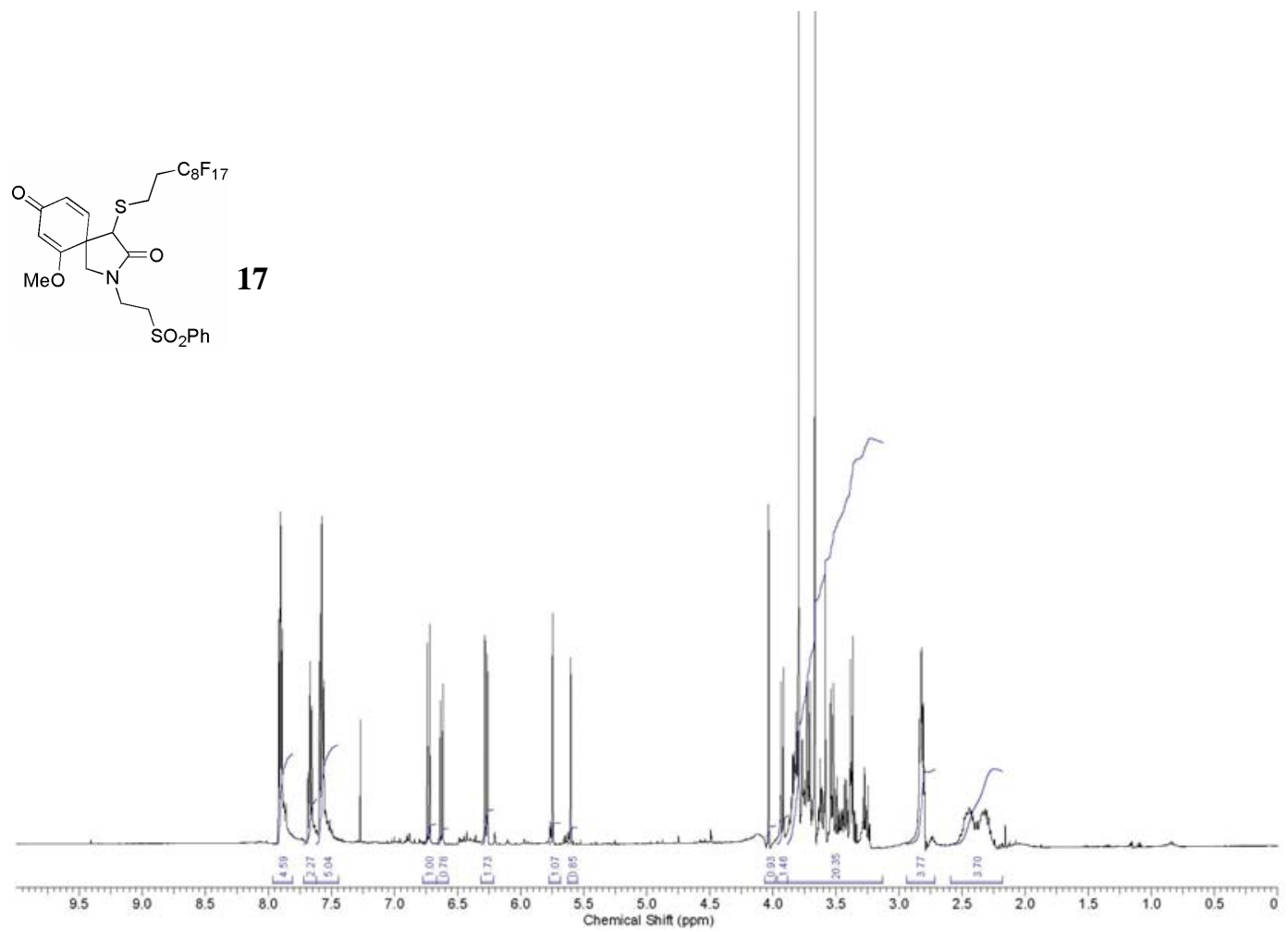

$75 \mathrm{MHz}\left(\mathrm{CDCl}_{3}\right)$

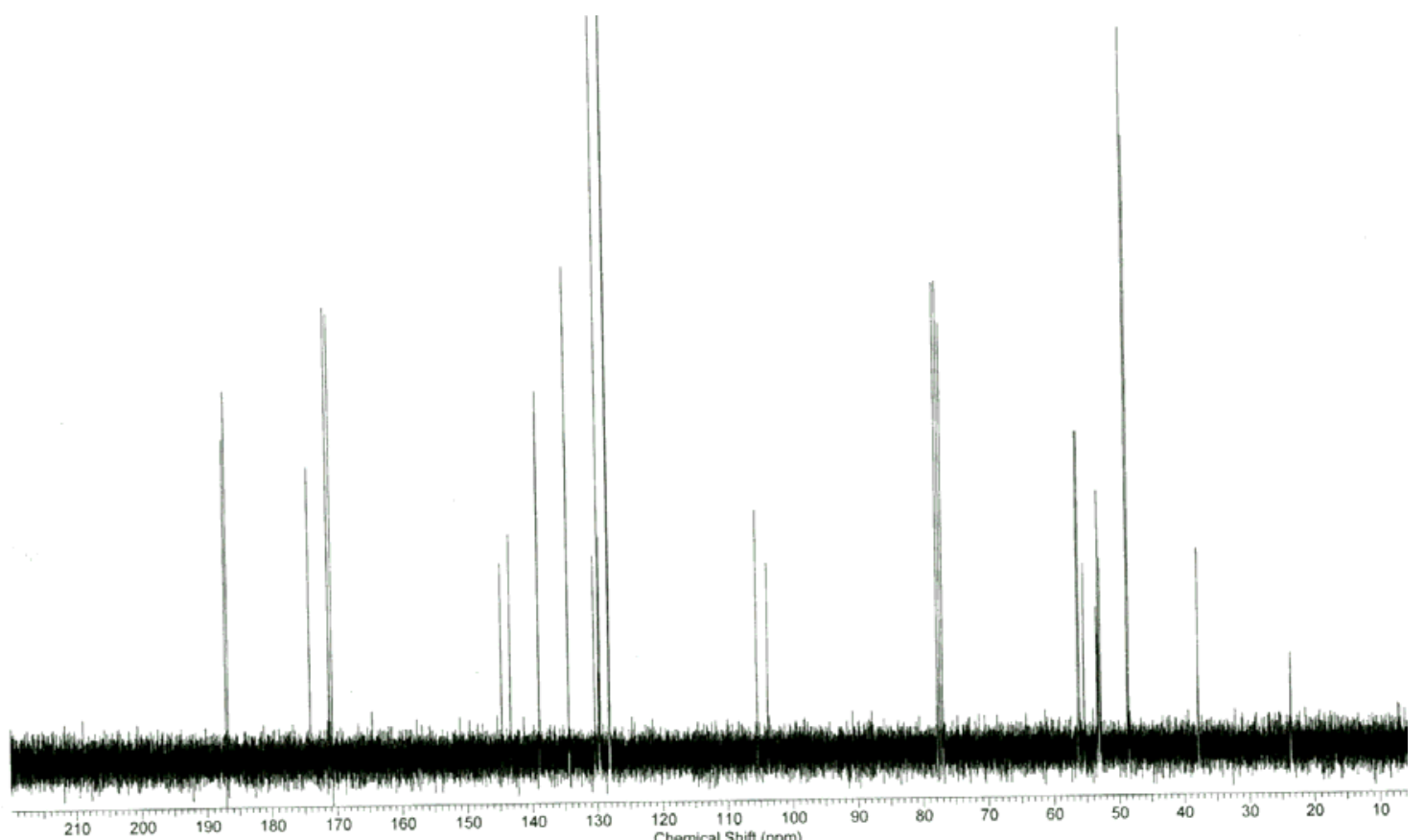


$500 \mathrm{MHz}\left(\mathrm{CDCl}_{3}\right)$

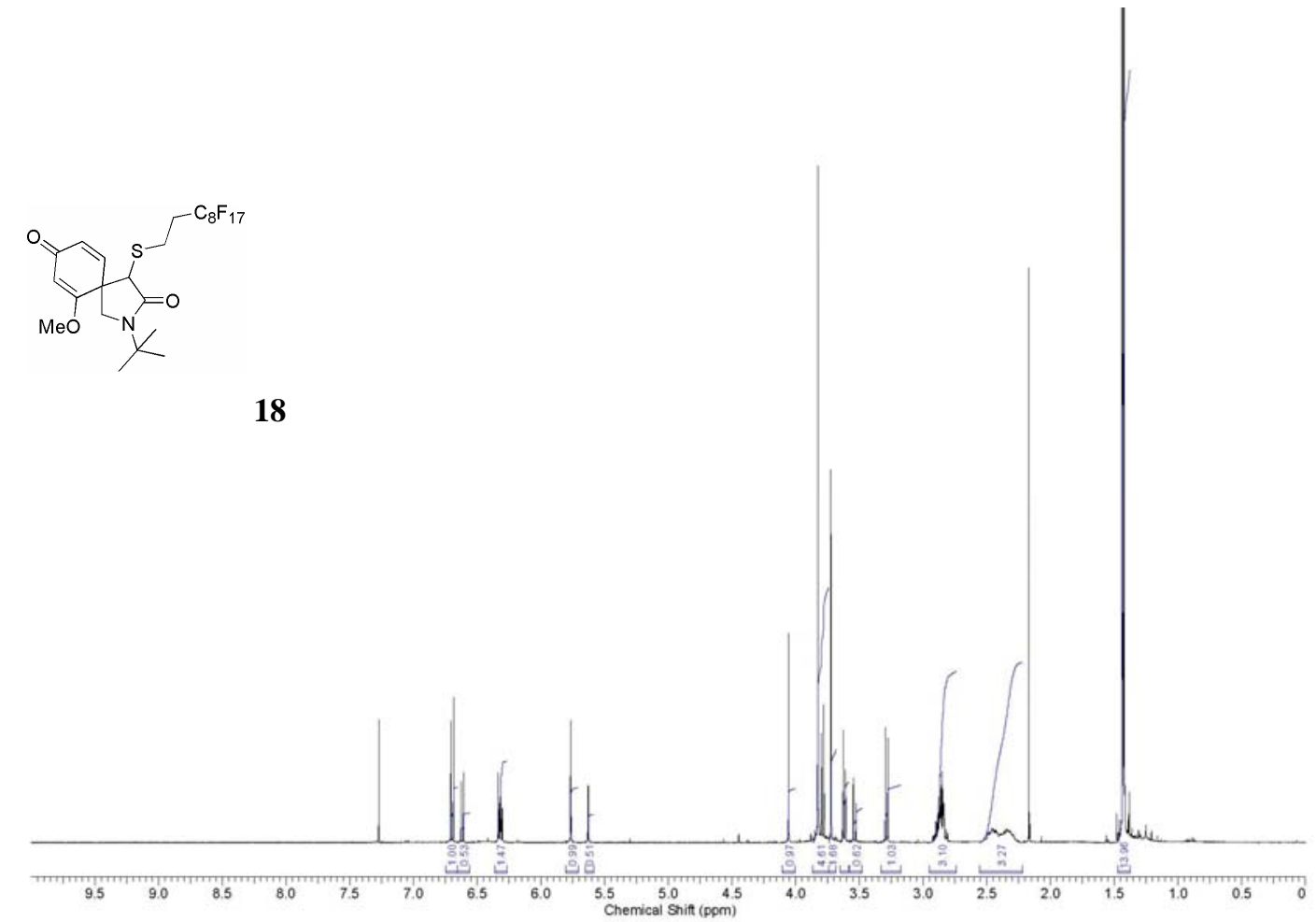

$125 \mathrm{MHz}\left(\mathrm{CDCl}_{3}\right)$

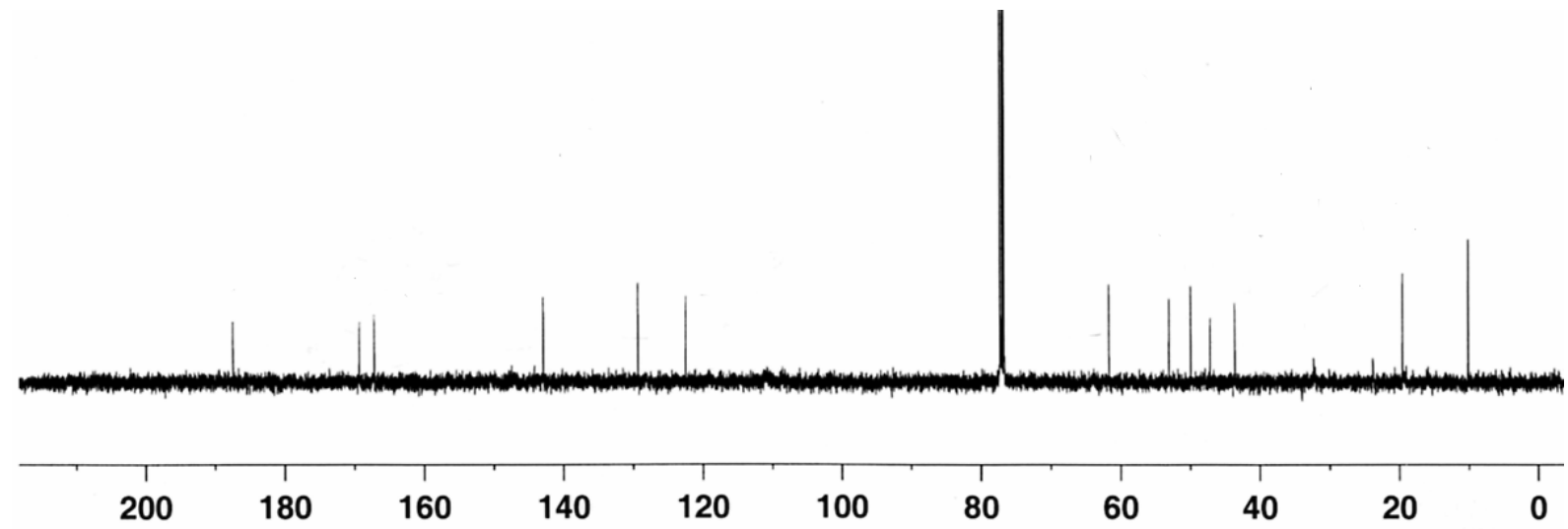


$300 \mathrm{MHz}\left(\mathrm{CDCl}_{3}\right)$

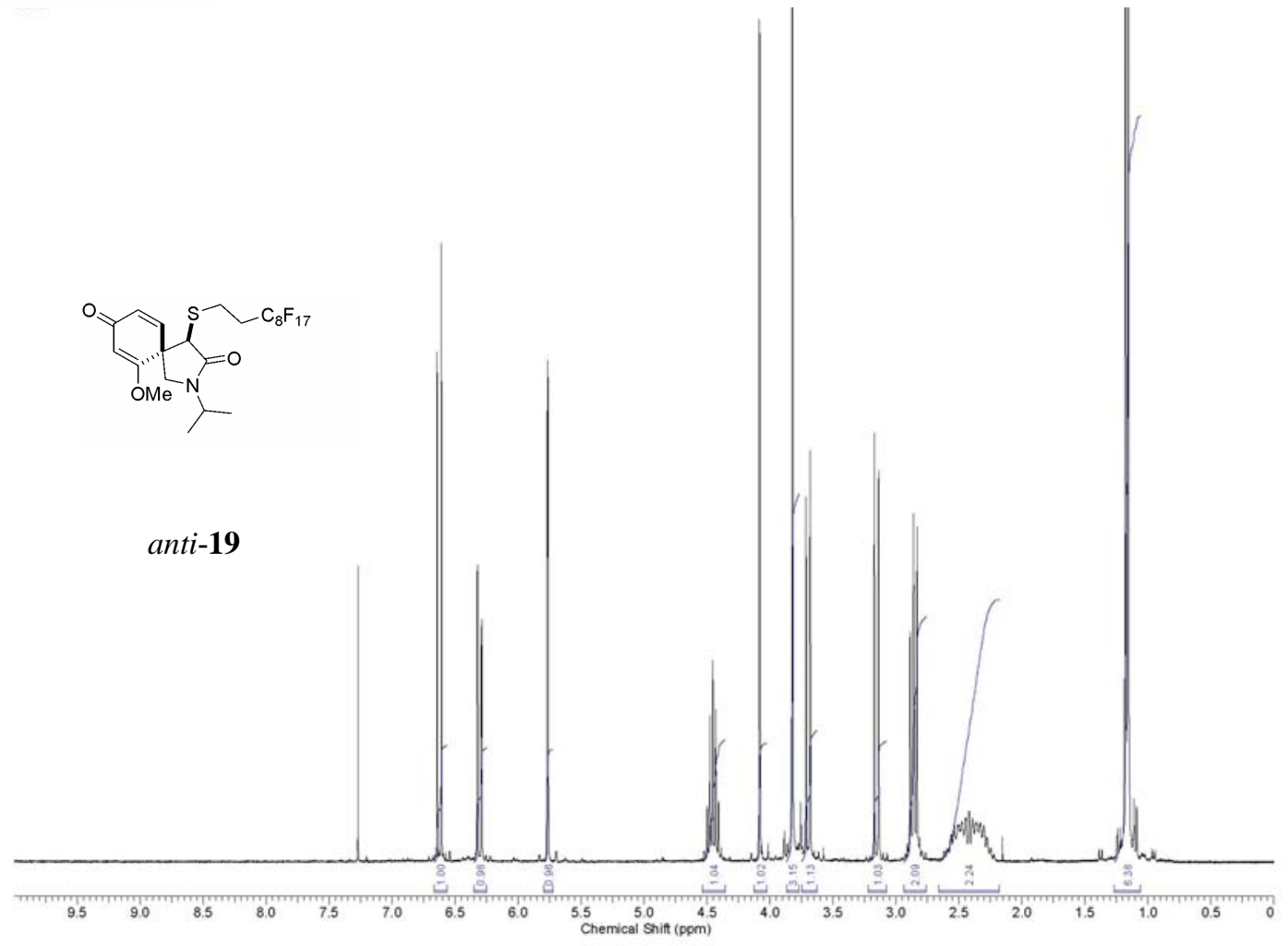

$100 \mathrm{MHz}\left(\mathrm{CDCl}_{3}\right)$

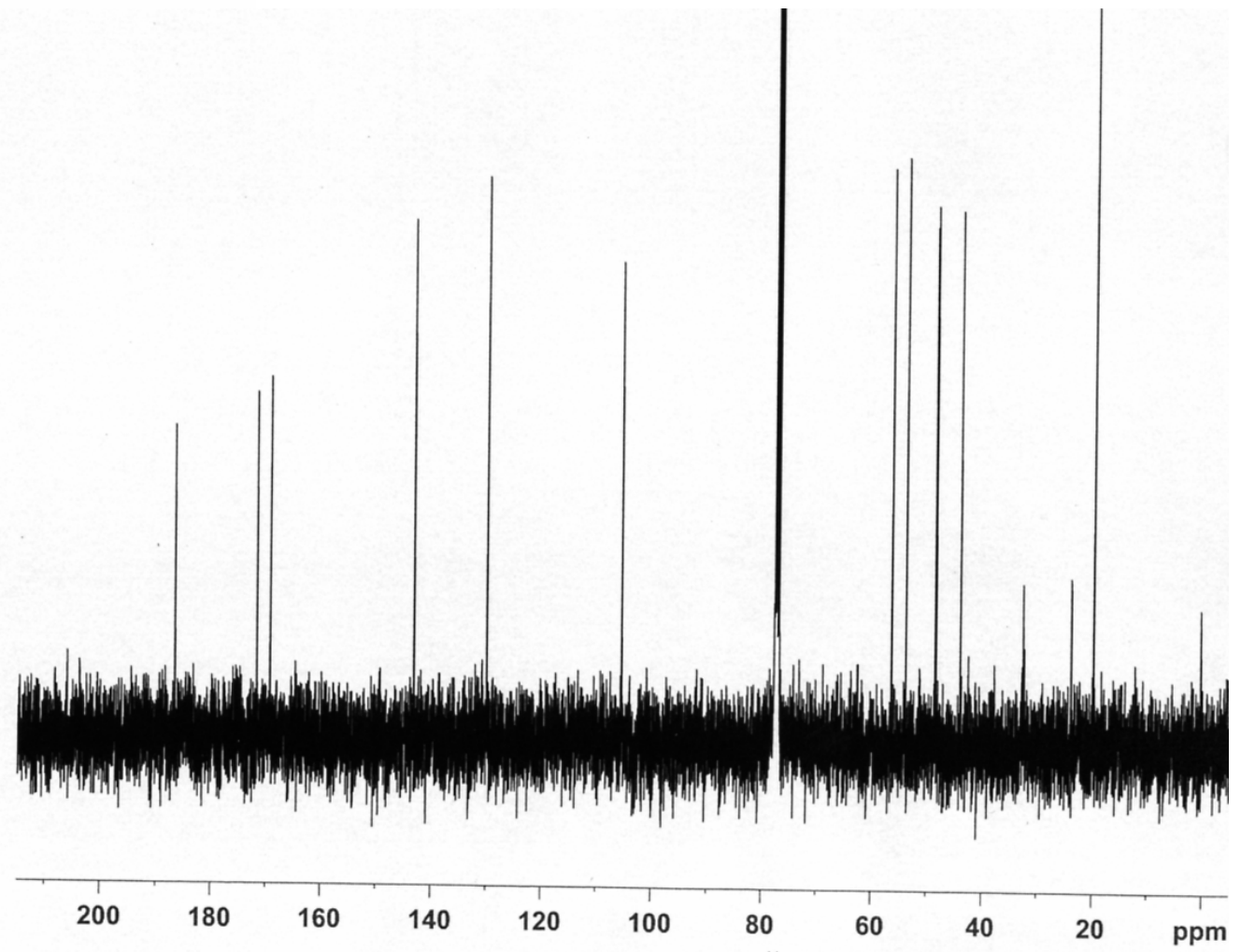


$300 \mathrm{MHz}\left(\mathrm{CDCl}_{3}\right)$

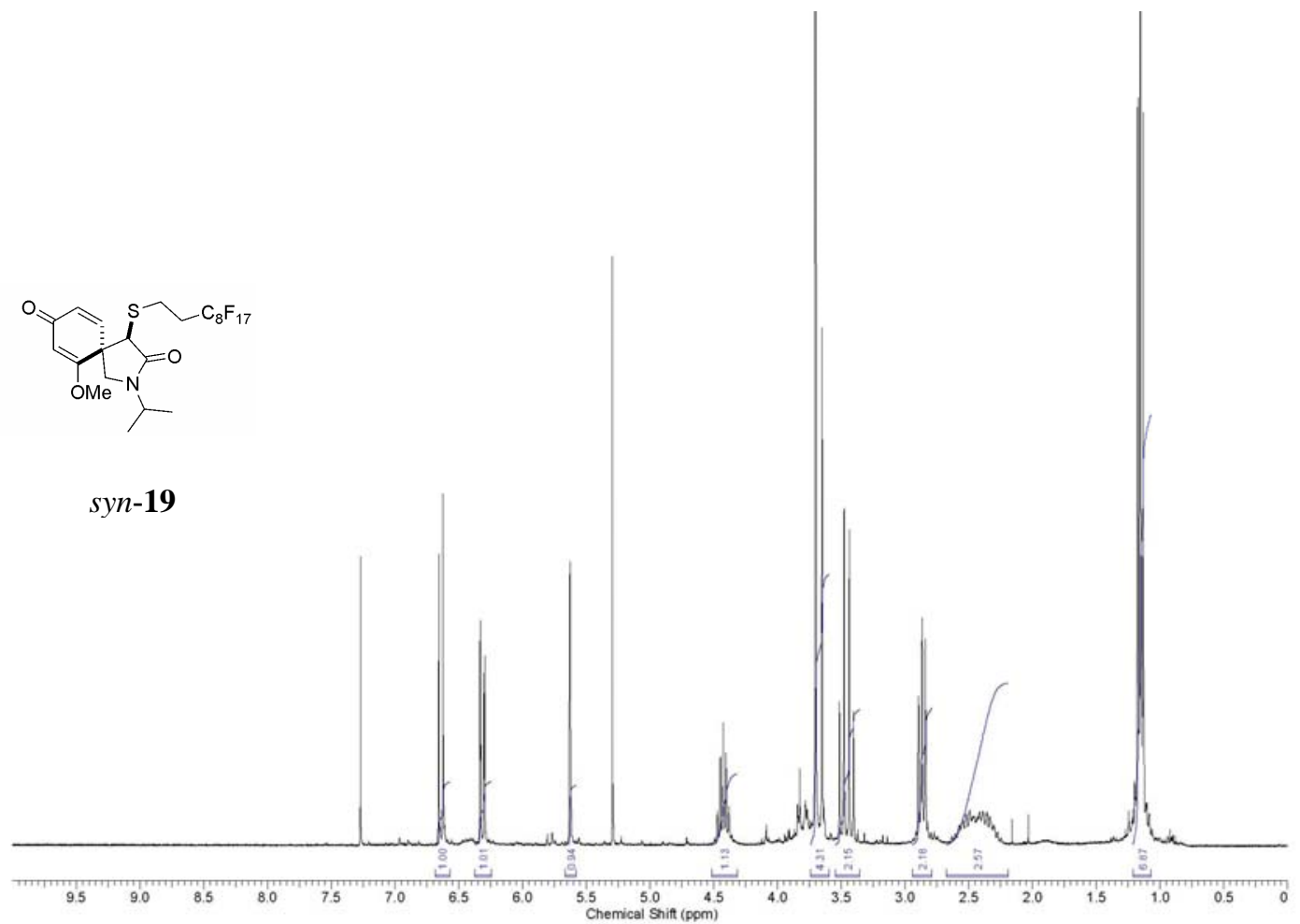

$100 \mathrm{MHz}\left(\mathrm{CDCl}_{3}\right)$

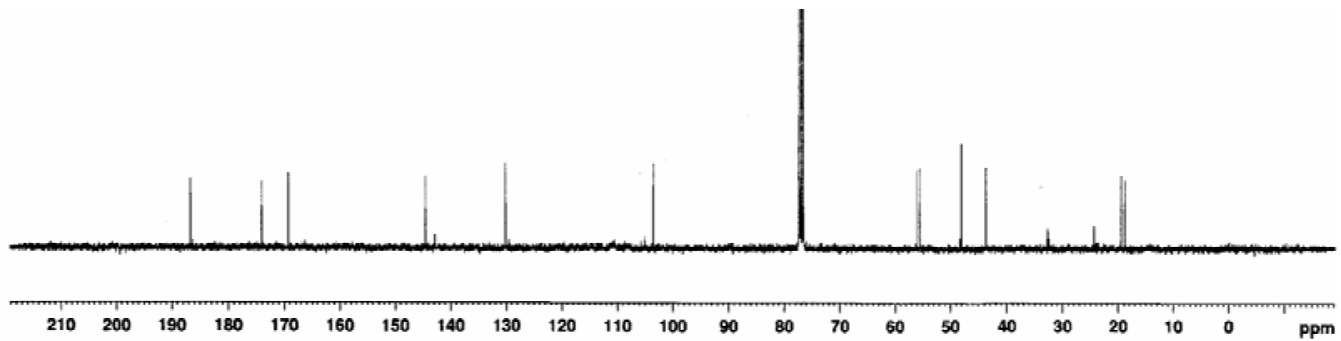


$300 \mathrm{MHz}\left(\mathrm{CDCl}_{3}\right)$

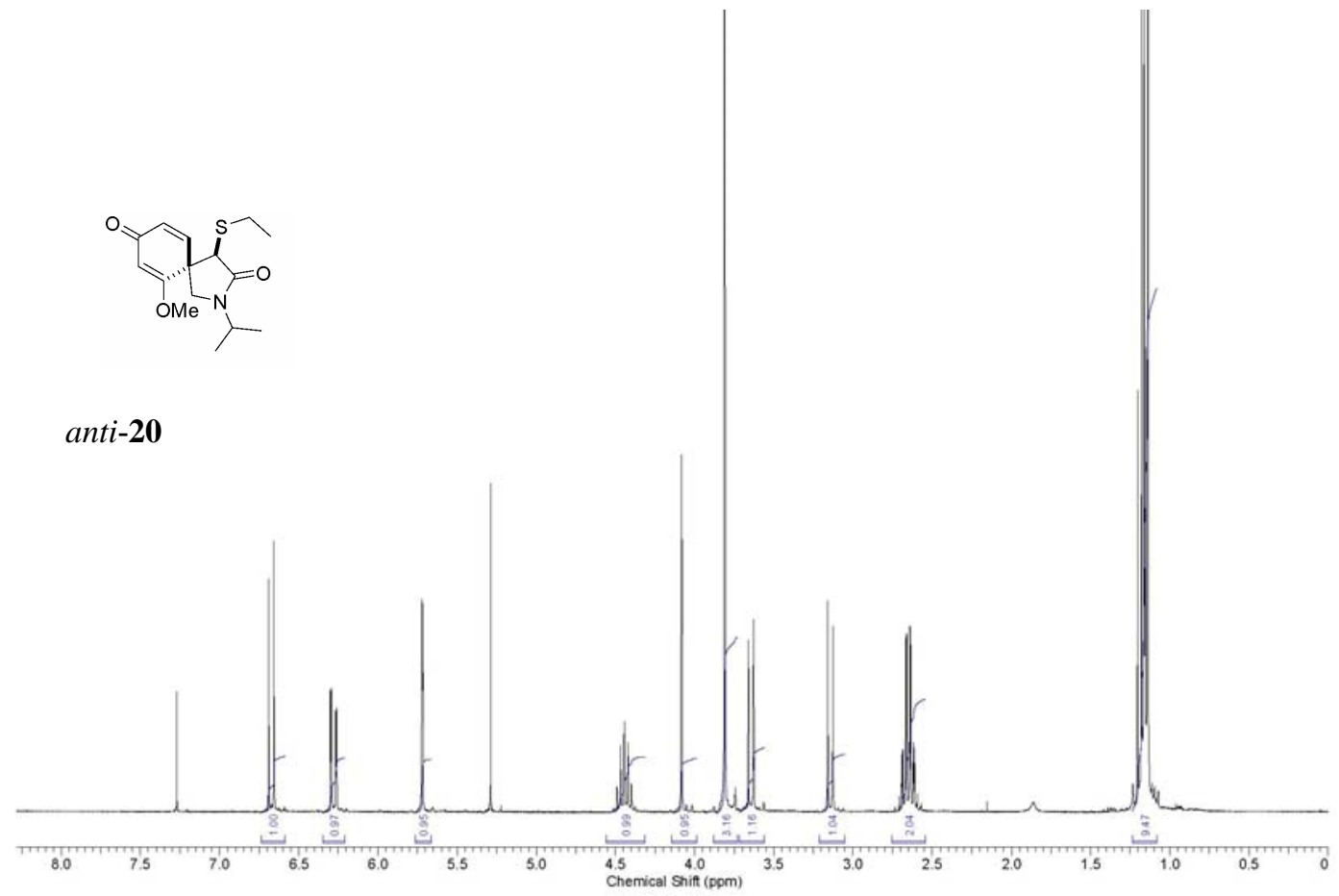

$75 \mathrm{MHz}\left(\mathrm{CDCl}_{3}\right)$

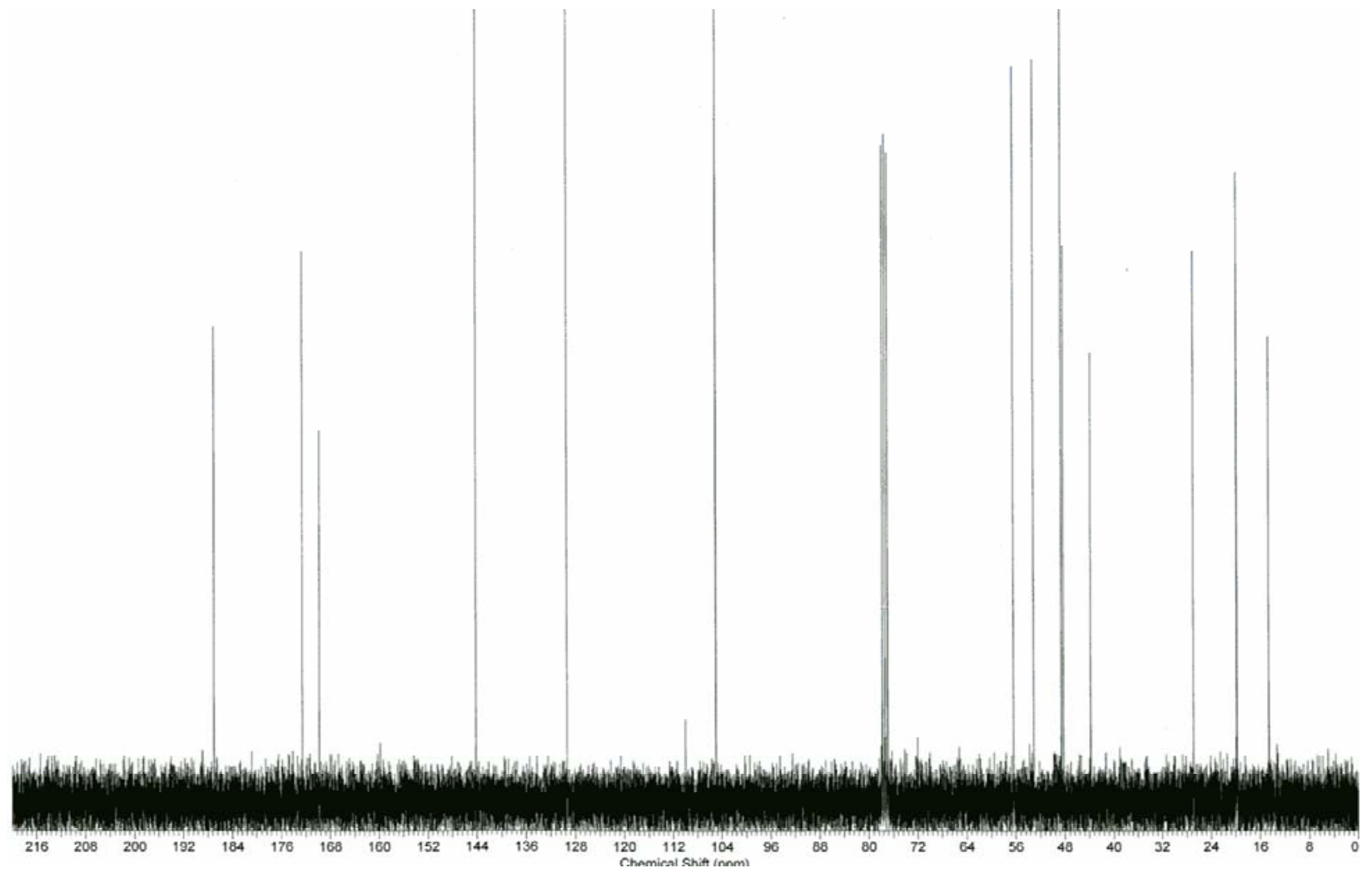


$300 \mathrm{MHz}\left(\mathrm{CDCl}_{3}\right)$

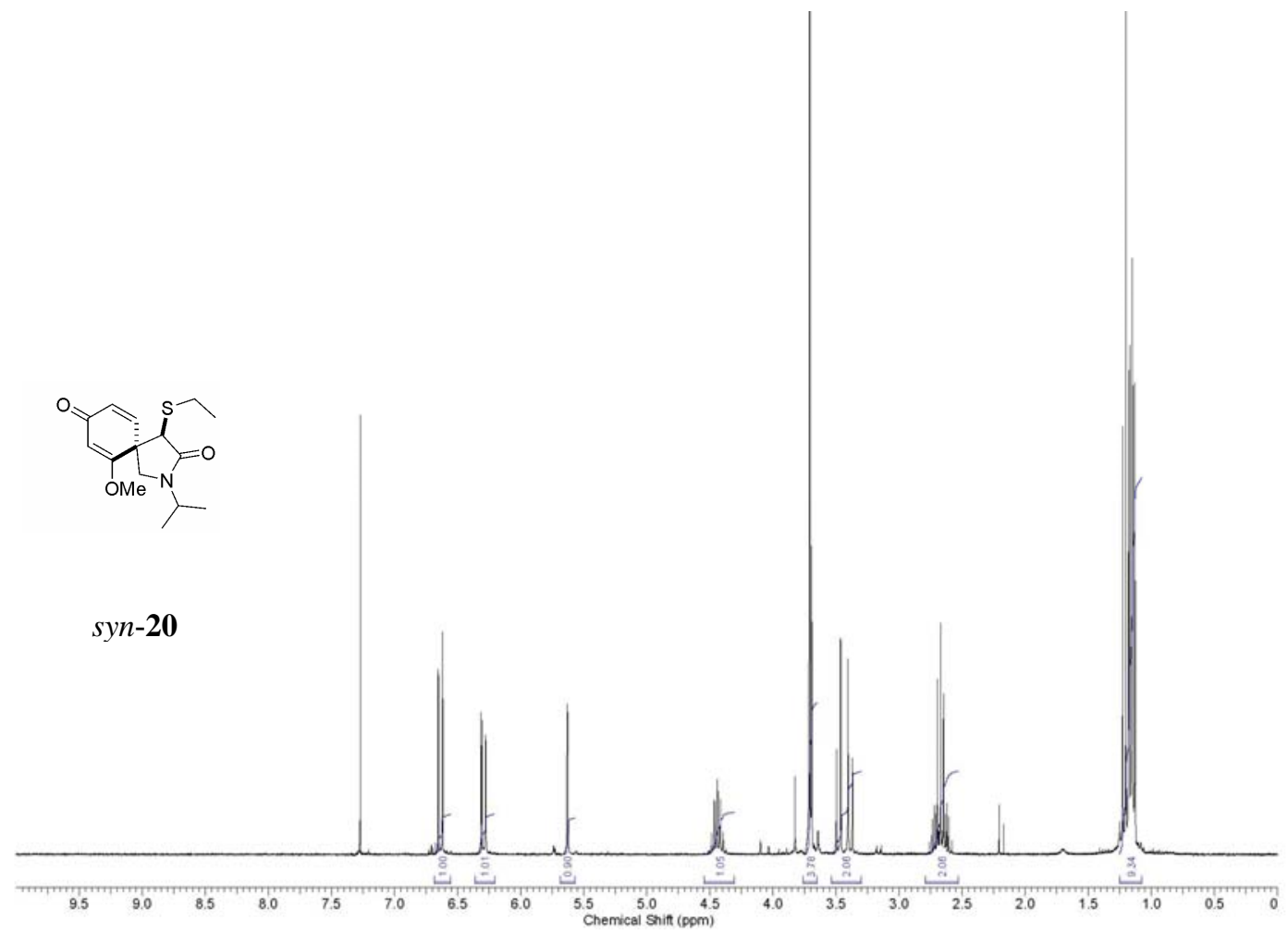

$75 \mathrm{MHz}\left(\mathrm{CDCl}_{3}\right)$

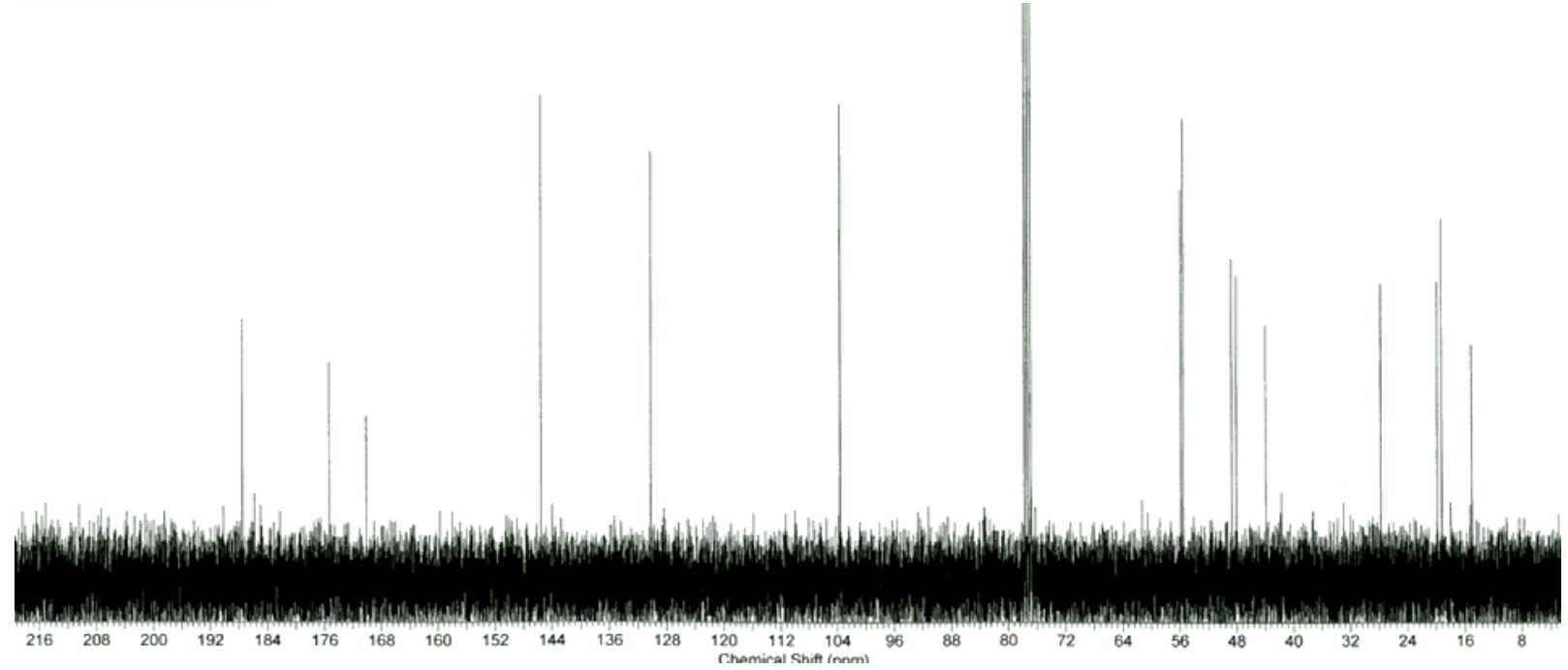


$500 \mathrm{MHz}\left(\mathrm{CDCl}_{3}\right)$

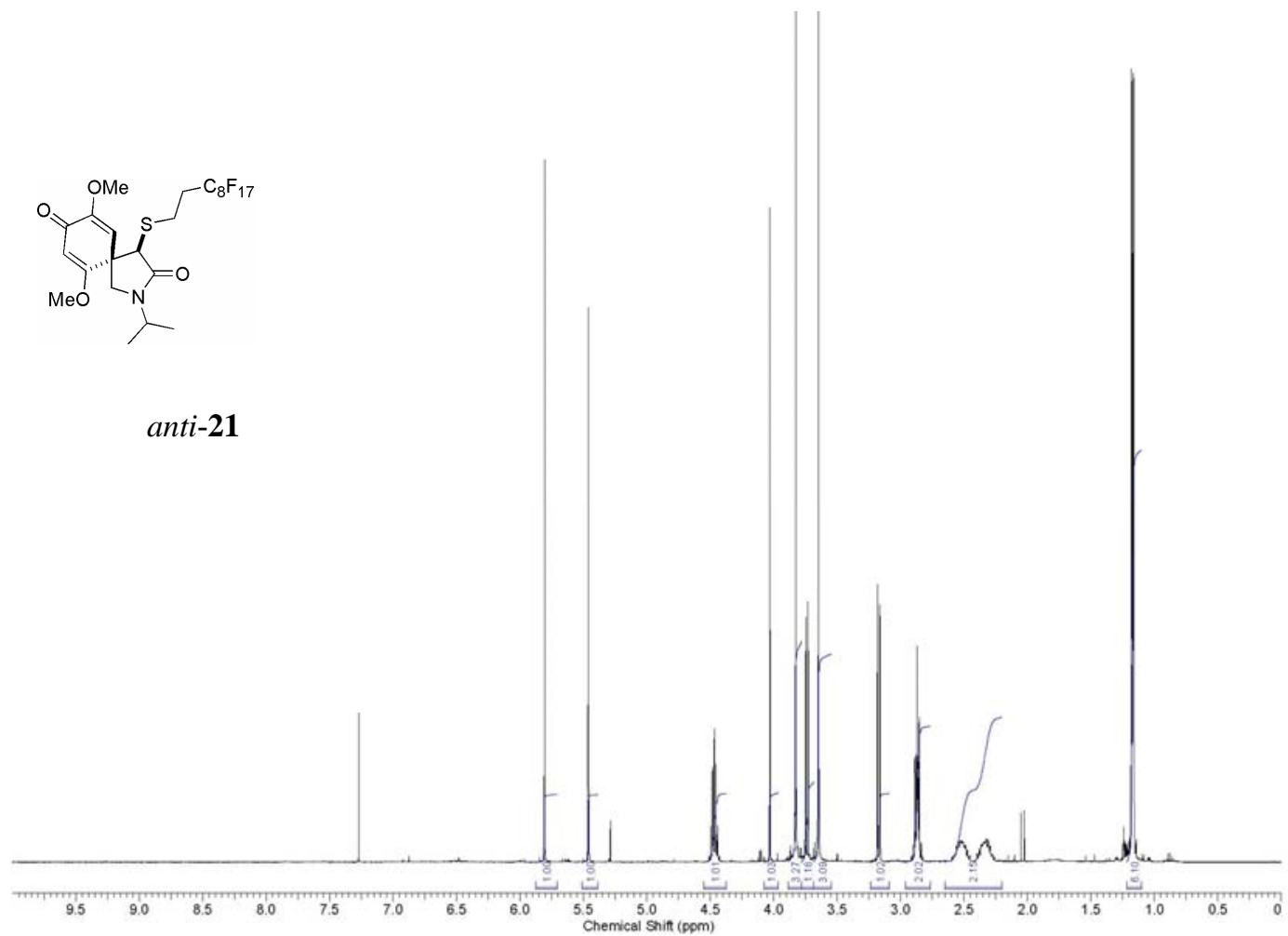

$125 \mathrm{MHz}\left(\mathrm{CDCl}_{3}\right)$

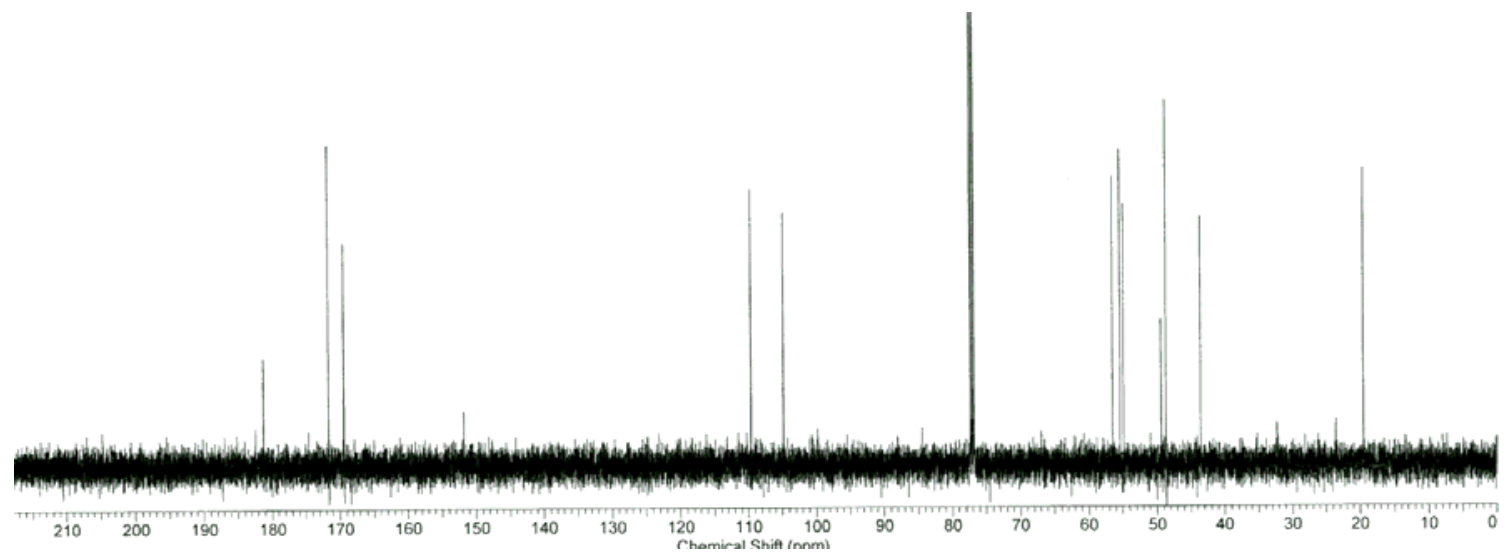


$500 \mathrm{MHz}\left(\mathrm{CDCl}_{3}\right)$

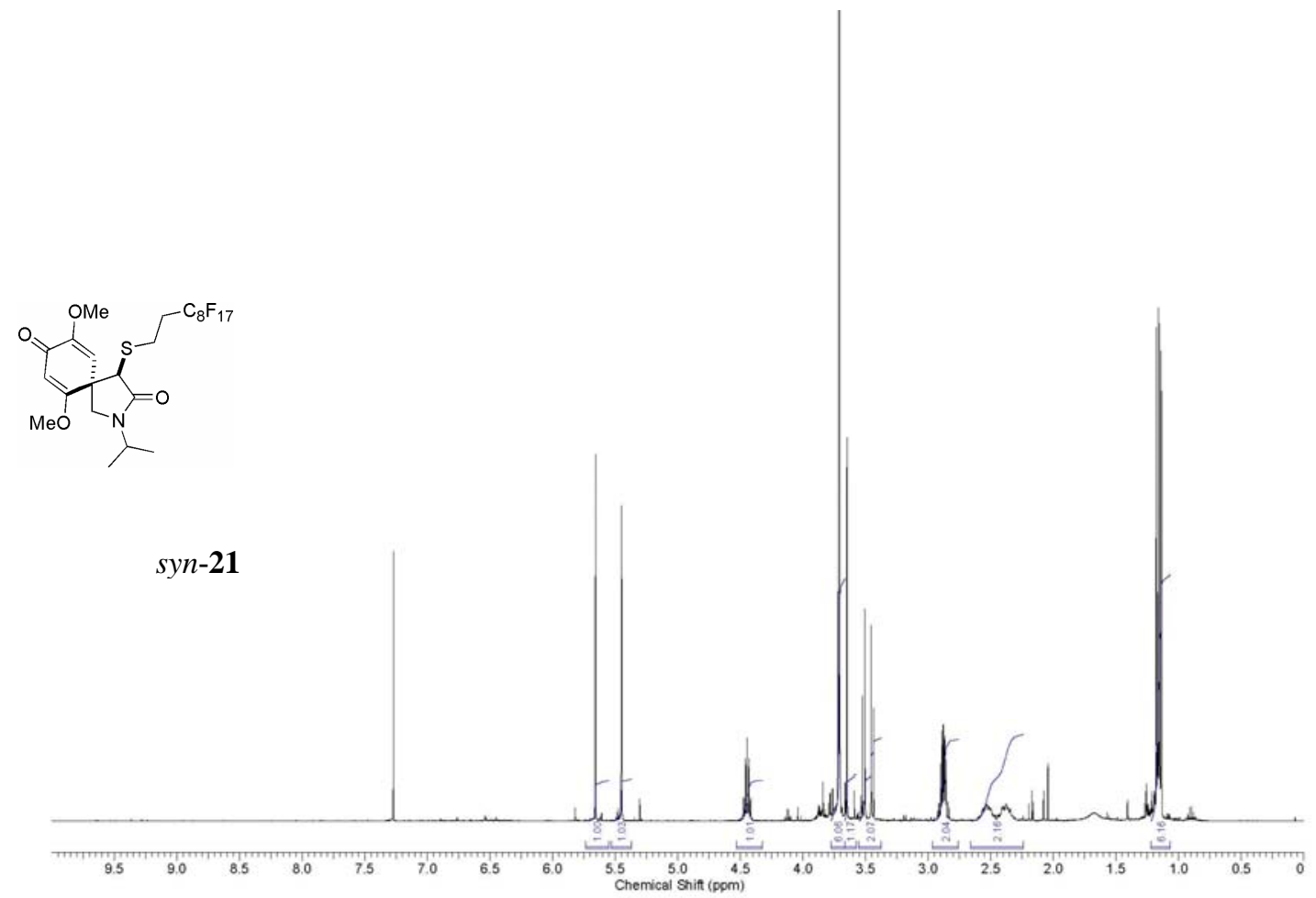

$100 \mathrm{MHz}\left(\mathrm{CDCl}_{3}\right)$

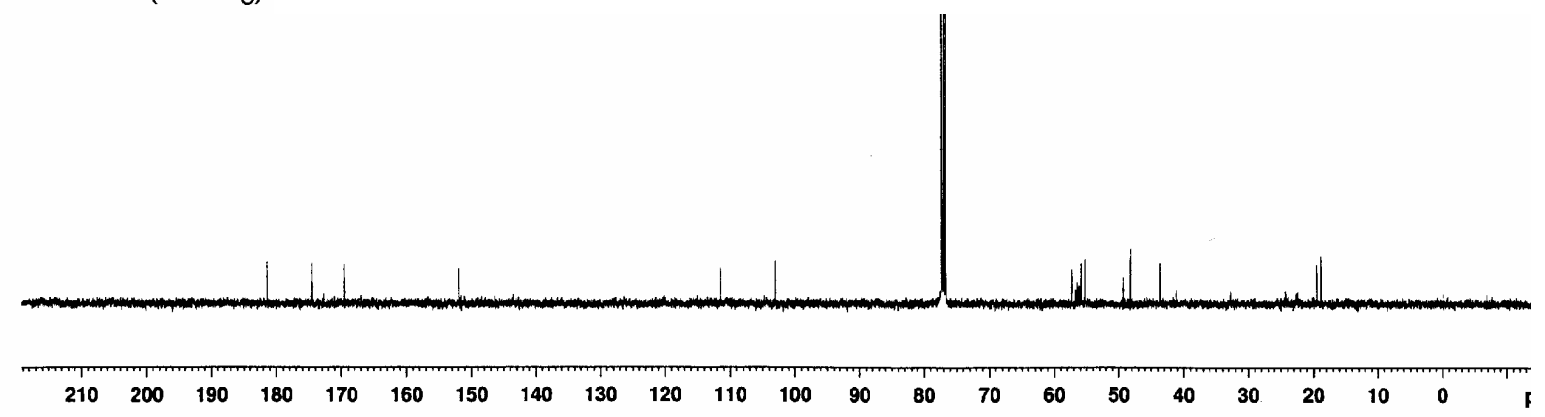




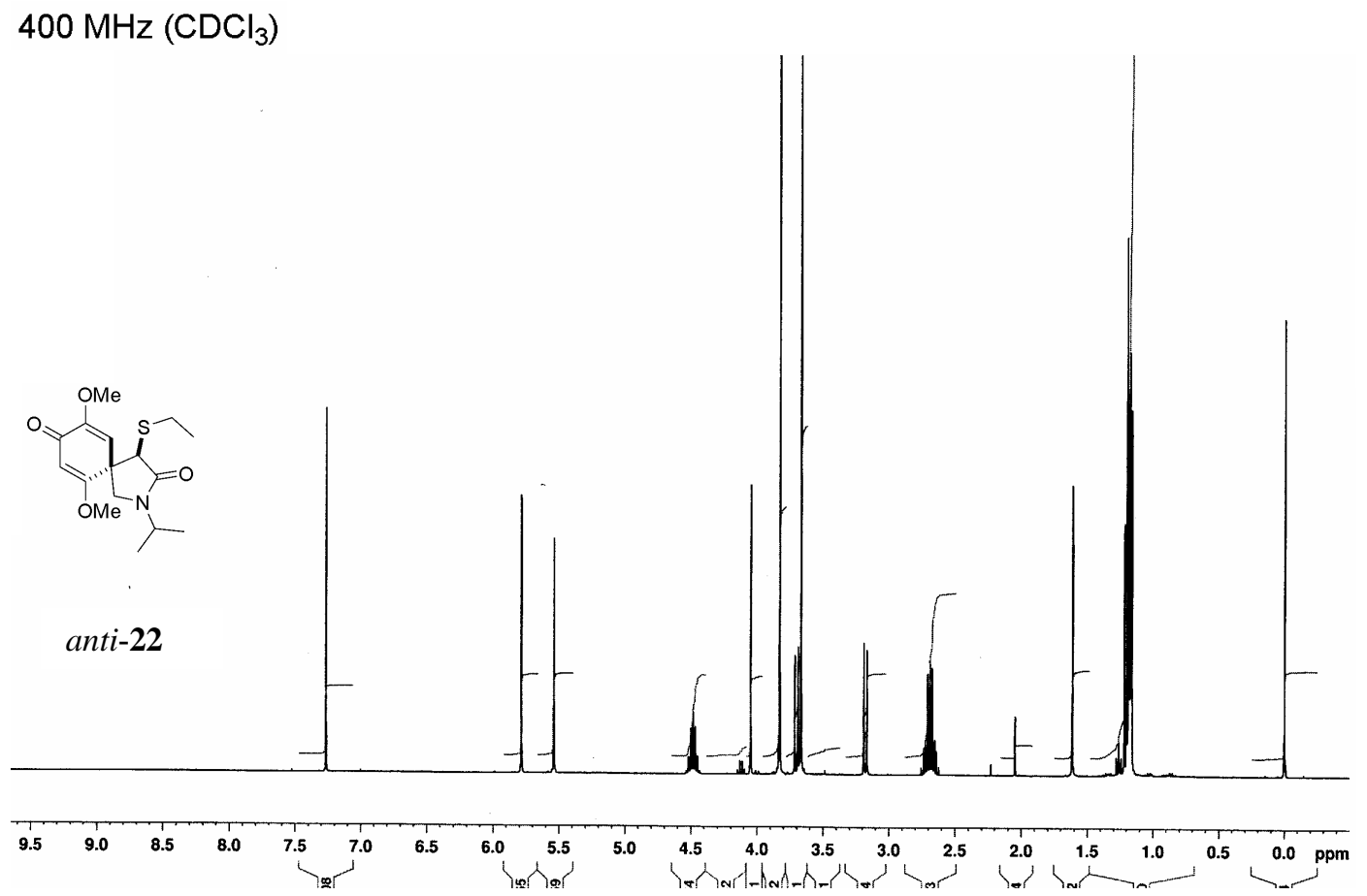

$100 \mathrm{MHz}\left(\mathrm{CDCl}_{3}\right)$

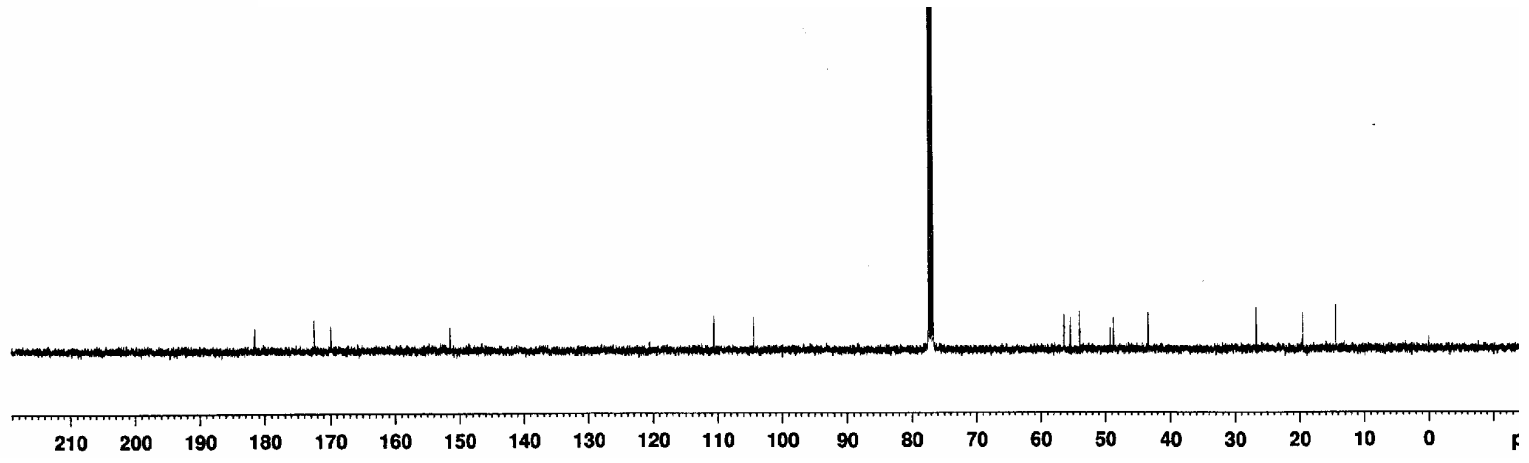




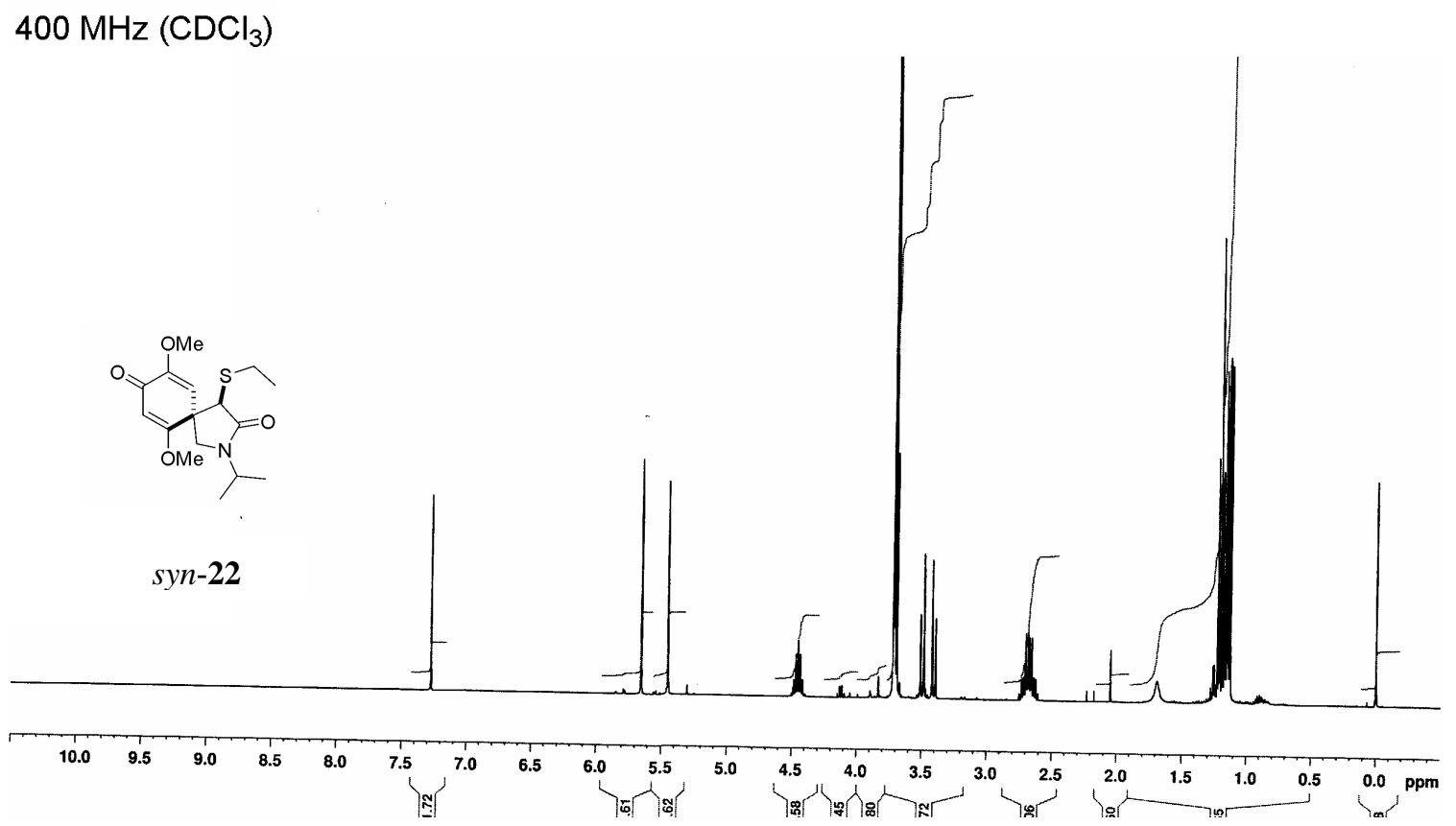

$100 \mathrm{MHz}\left(\mathrm{CDCl}_{3}\right)$

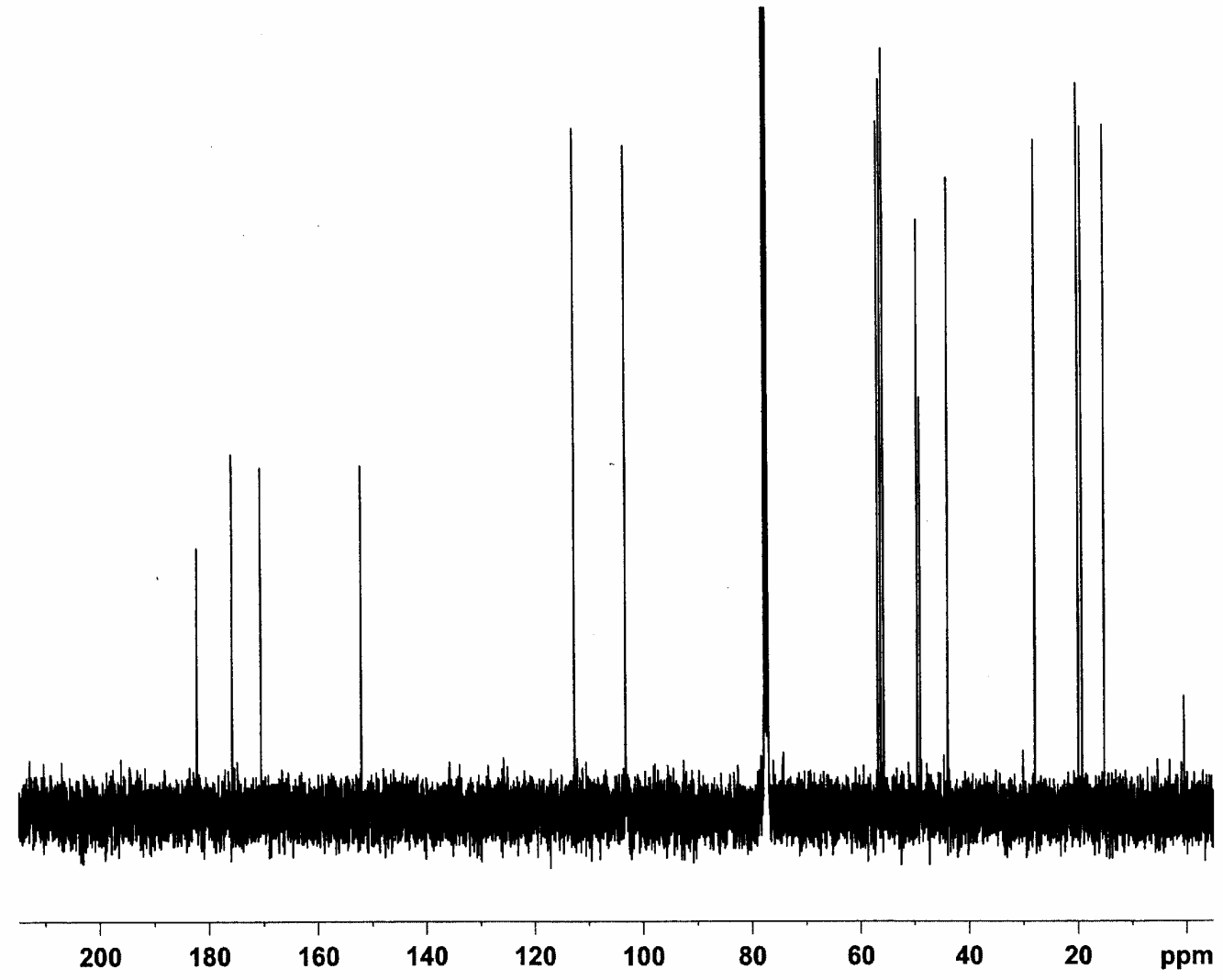


$500 \mathrm{MHz}\left(\mathrm{CDCl}_{3}\right)$

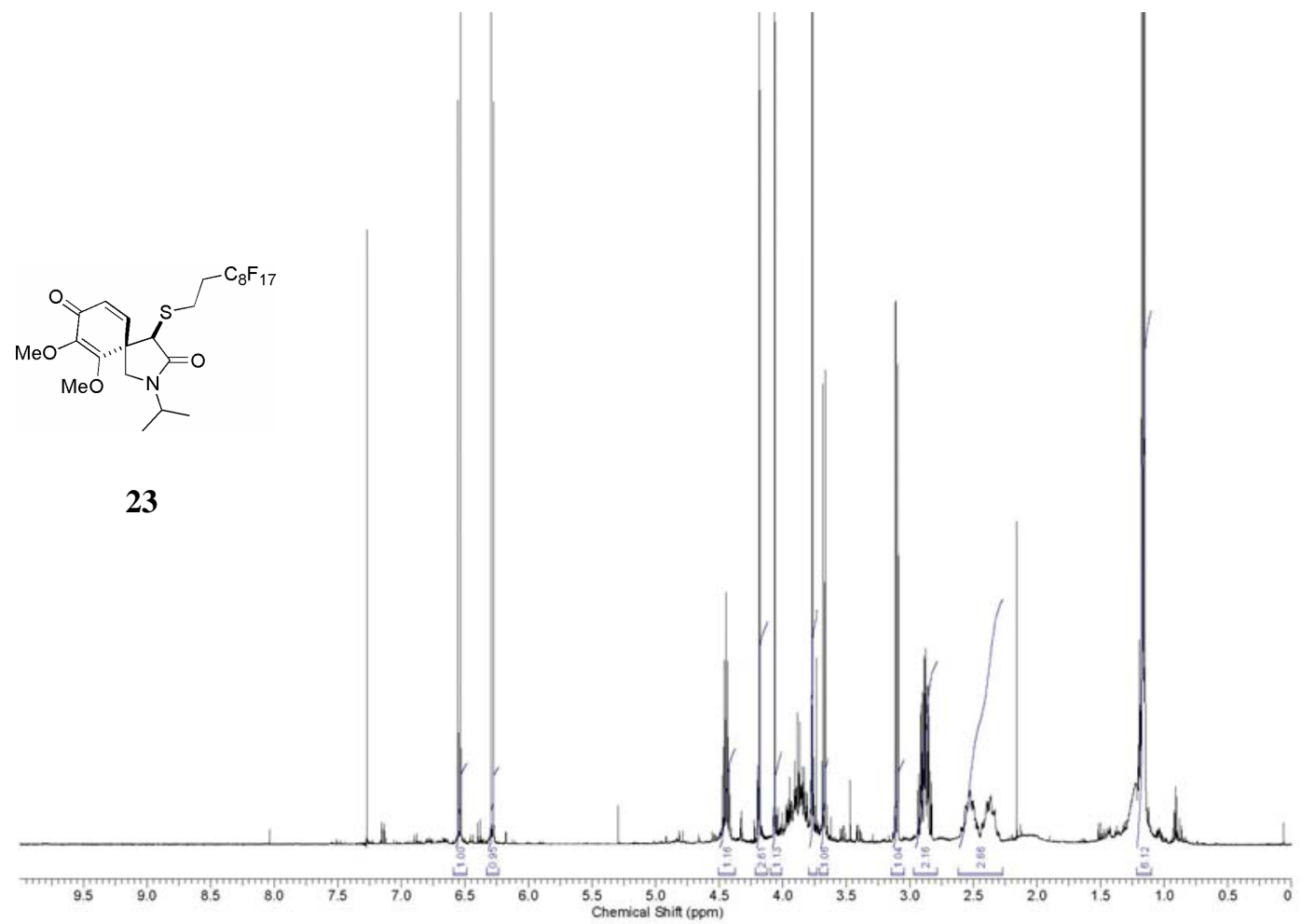

$125 \mathrm{MHz}\left(\mathrm{CDCl}_{3}\right)$

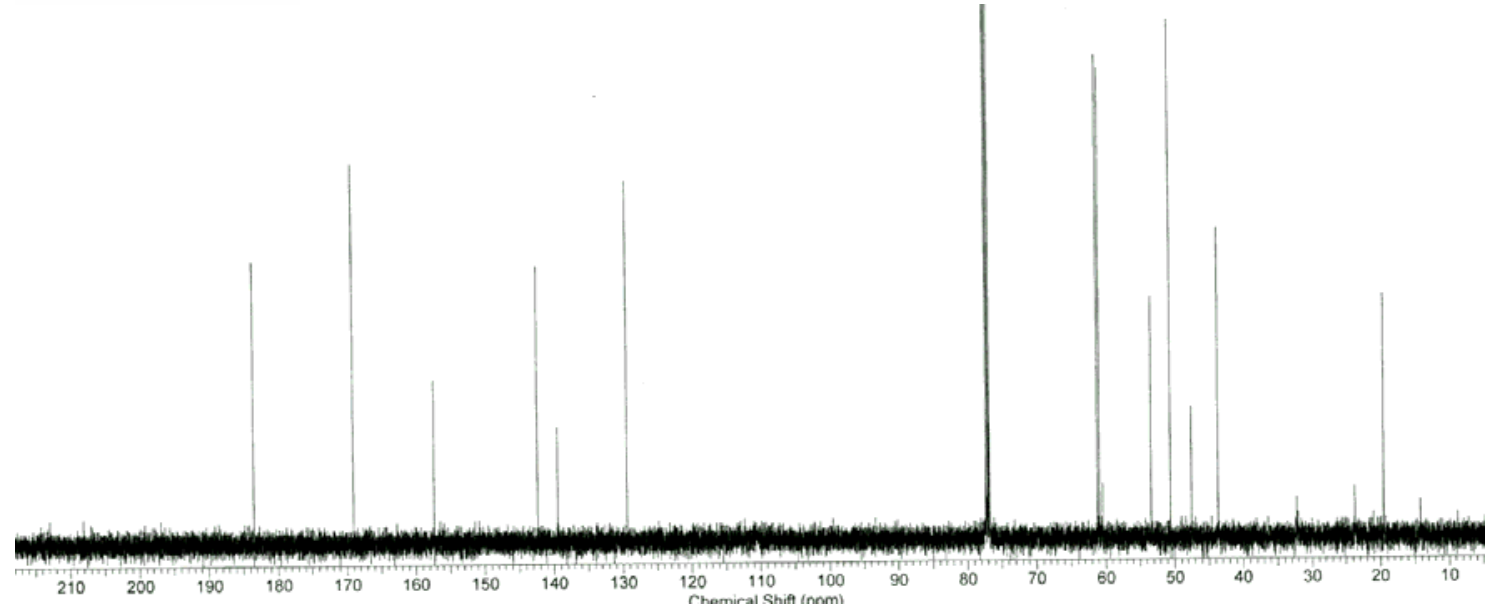


$500 \mathrm{MHz}\left(\mathrm{CDCl}_{3}\right)$

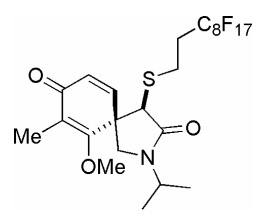

24

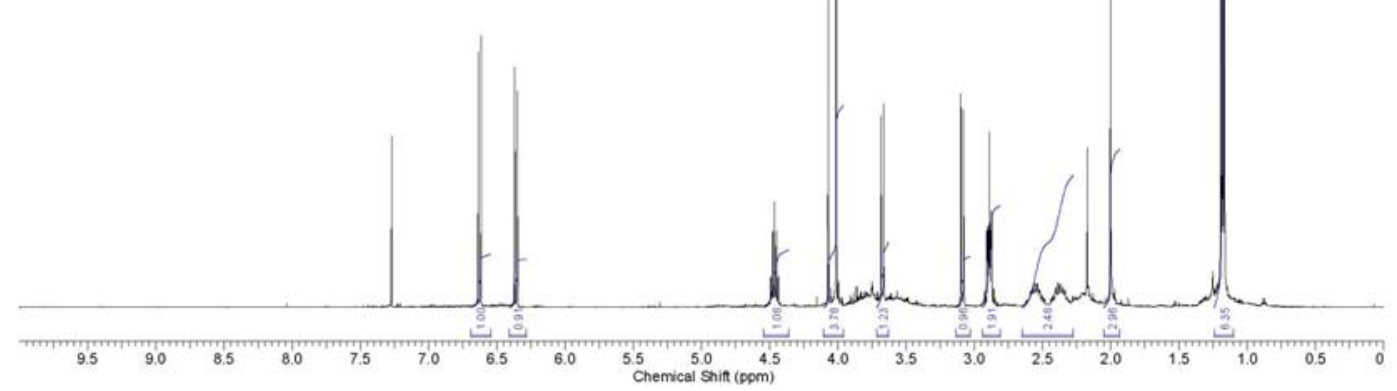

$100 \mathrm{MHz}\left(\mathrm{CDCl}_{3}\right)$

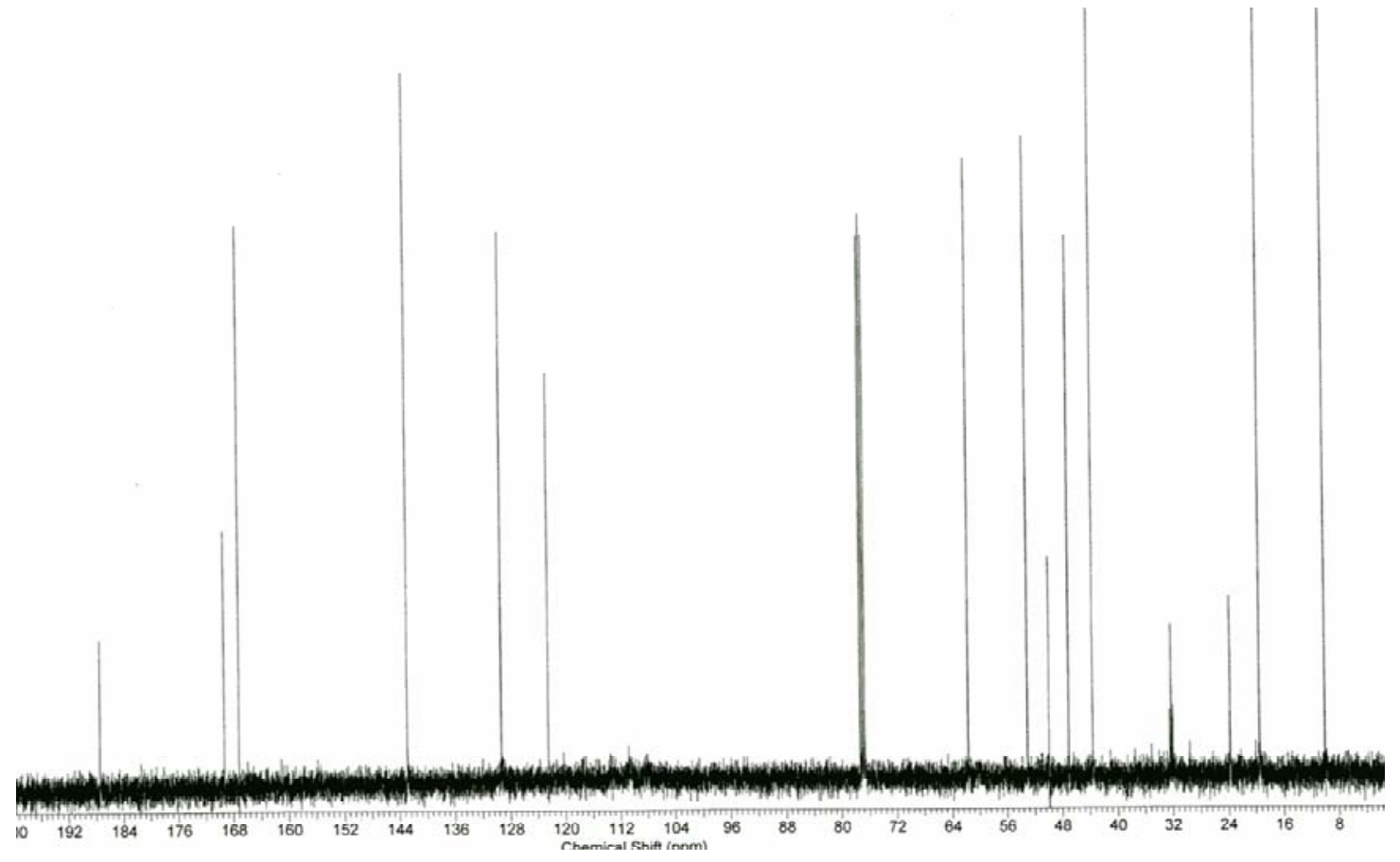


$500 \mathrm{MHz}\left(\mathrm{CDCl}_{3}\right)$

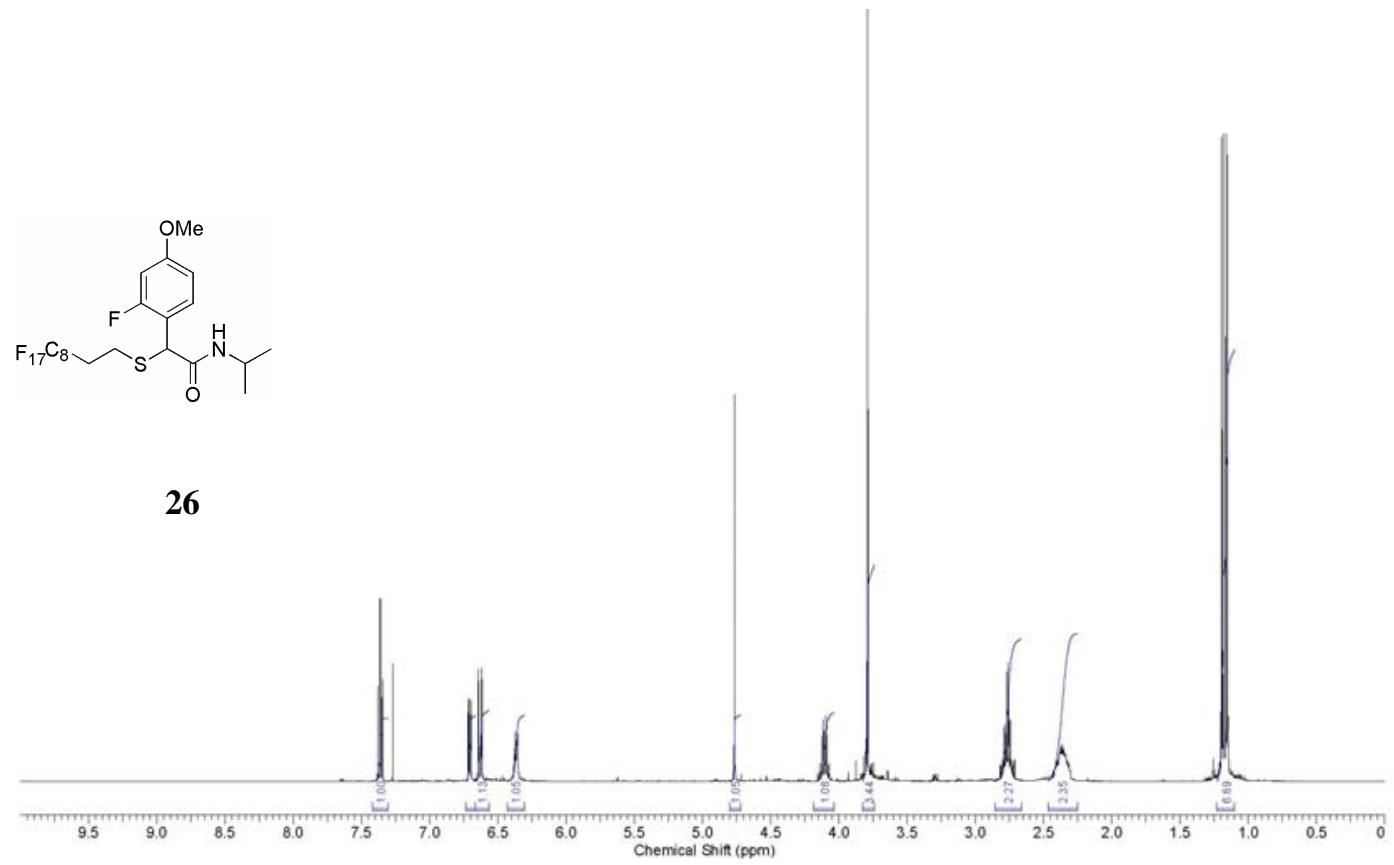

$125 \mathrm{MHz}\left(\mathrm{CDCl}_{3}\right)$

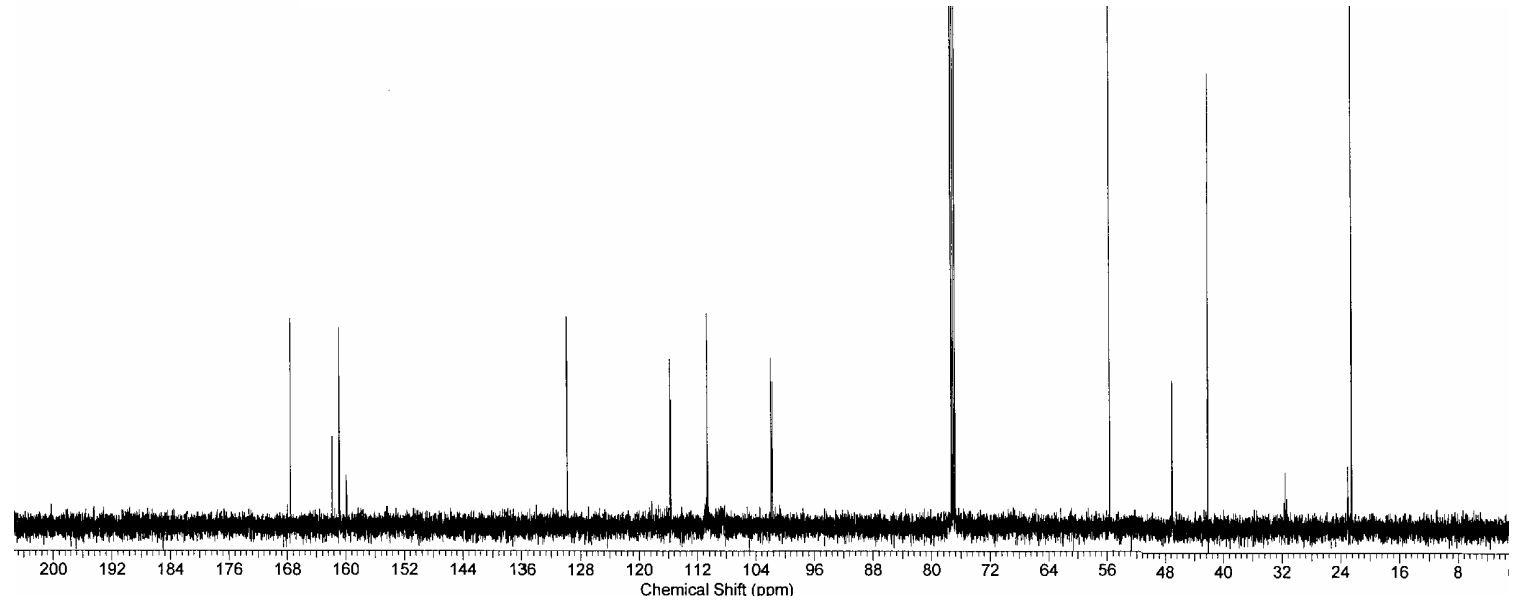


$500 \mathrm{MHz}\left(\mathrm{CDCl}_{3}\right)$

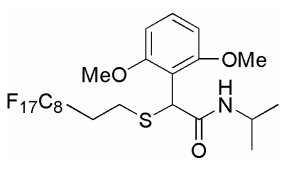

28

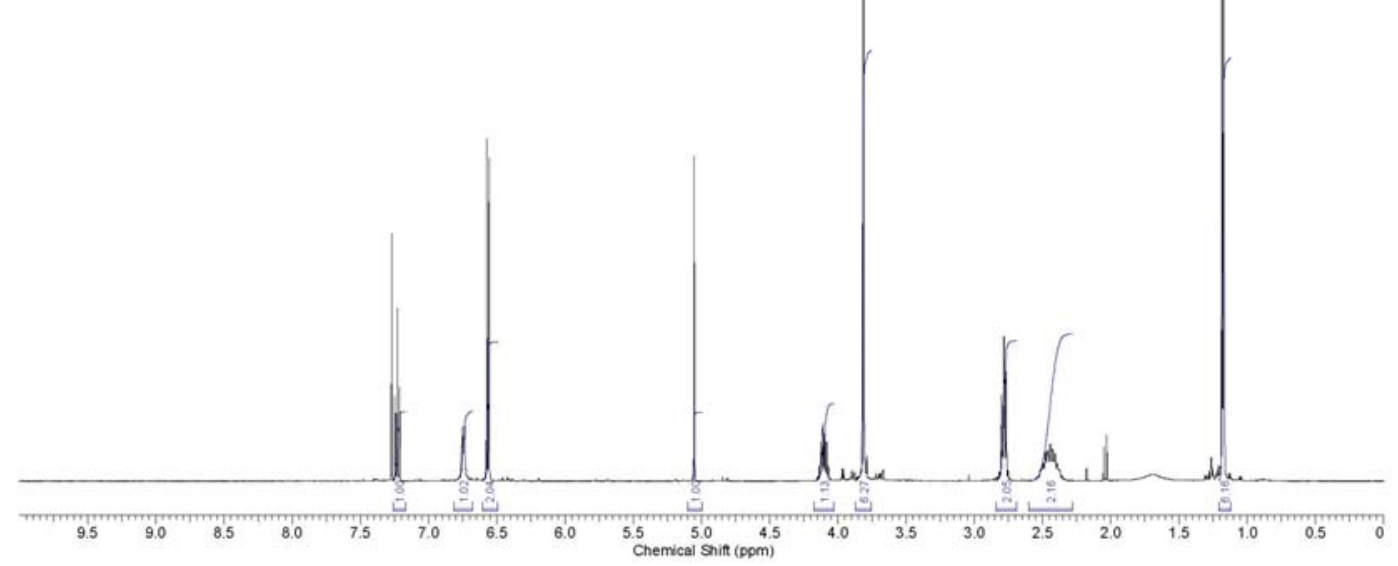

$125 \mathrm{MHz}\left(\mathrm{CDCl}_{3}\right)$

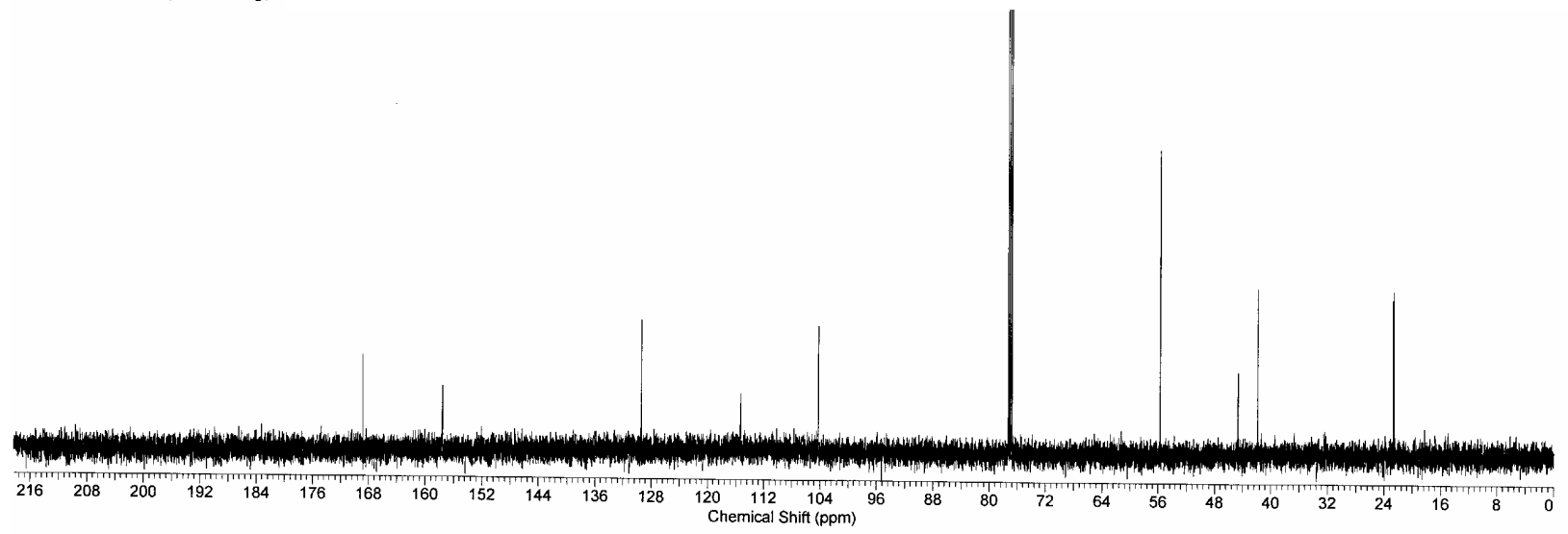




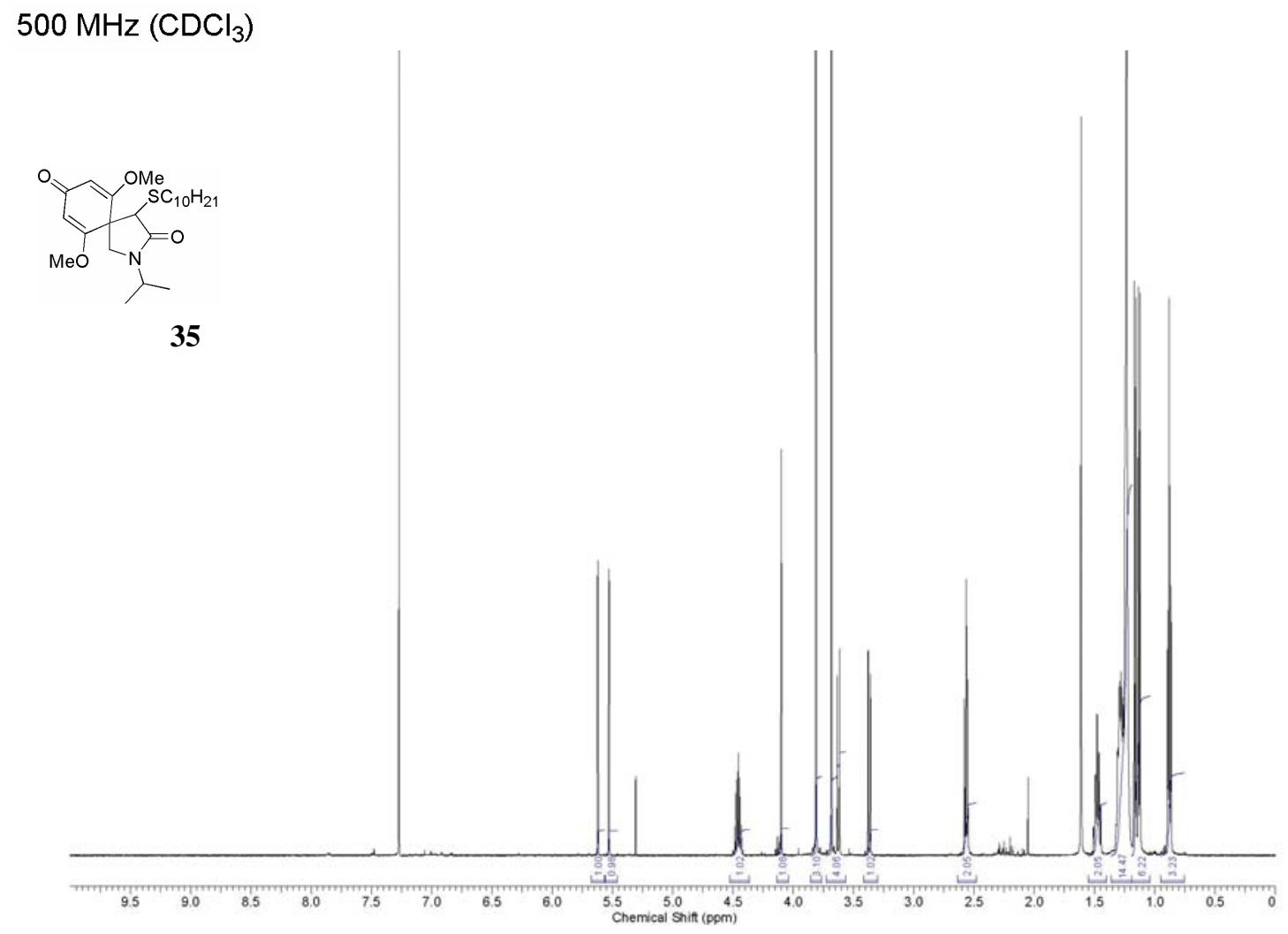

$100 \mathrm{MHz}\left(\mathrm{CDCl}_{3}\right)$

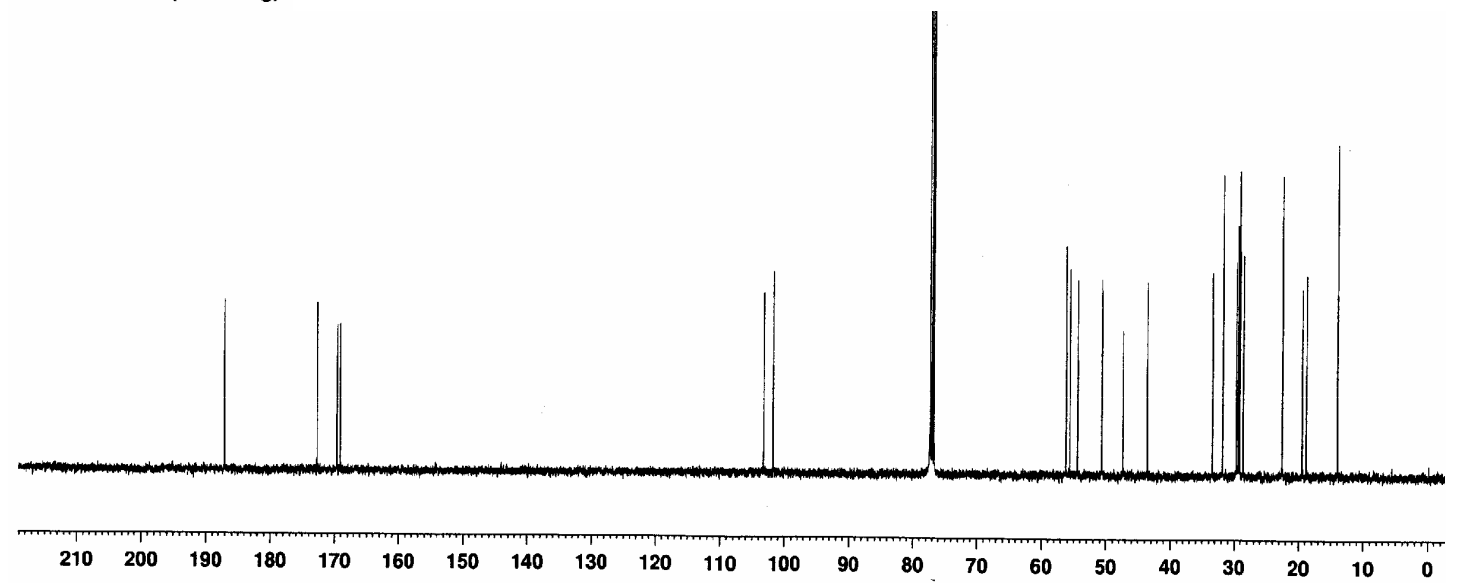


NMR spectra: modifications

$500 \mathrm{MHz}\left(\mathrm{CDCl}_{3}\right)$

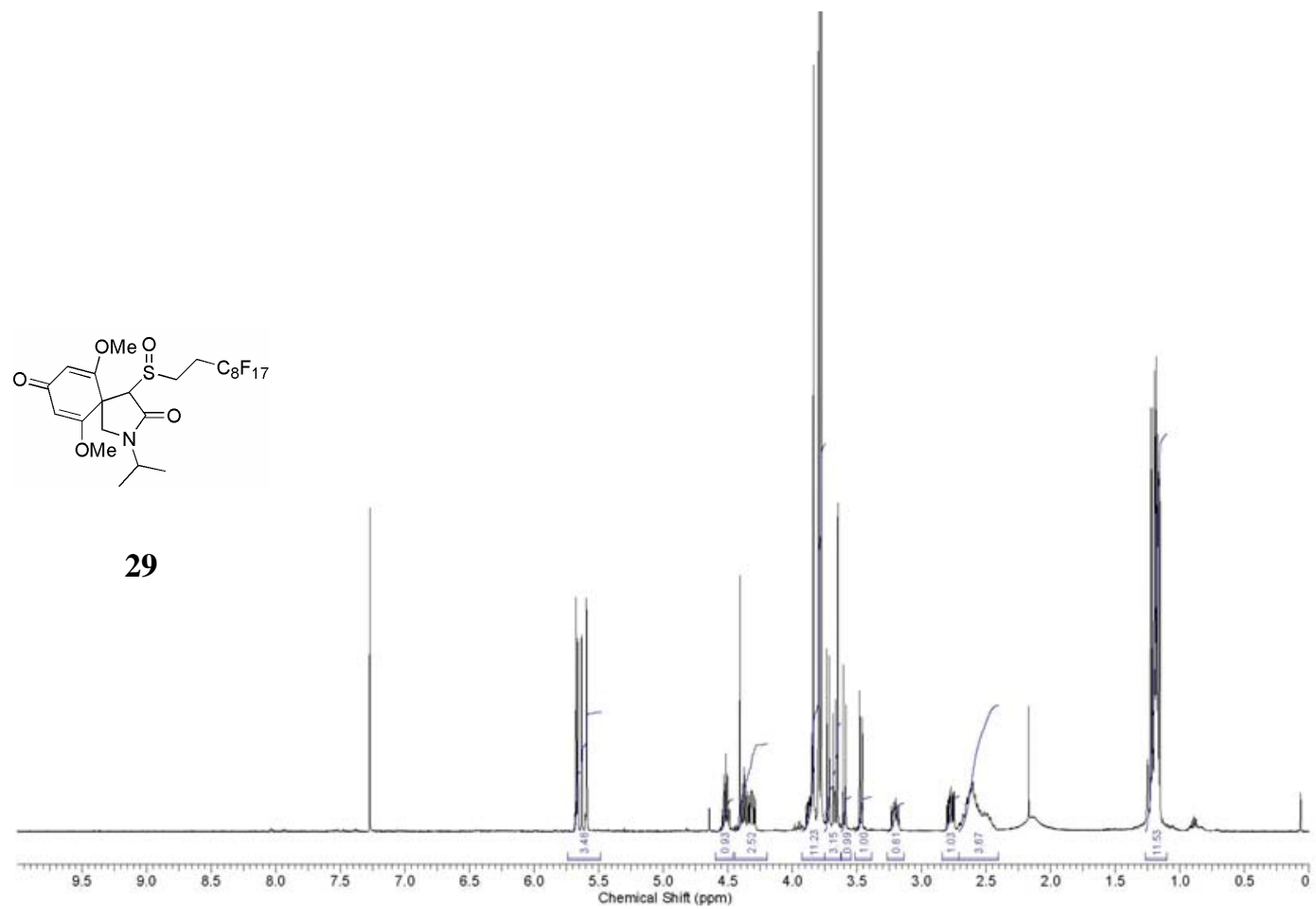

$125 \mathrm{MHz}\left(\mathrm{CDCl}_{3}\right)$

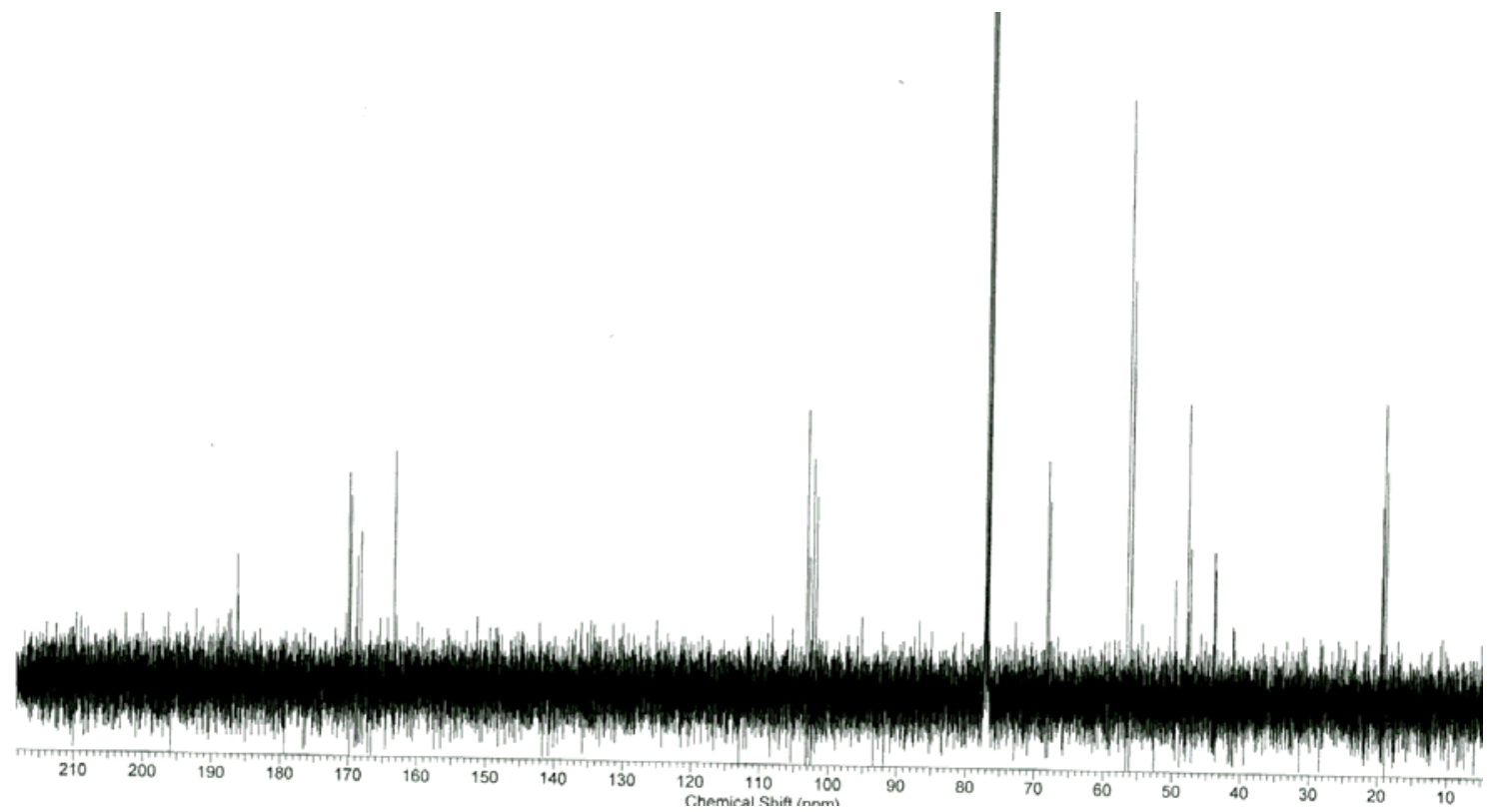


$500 \mathrm{MHz}\left(\mathrm{CDCl}_{3}\right)$

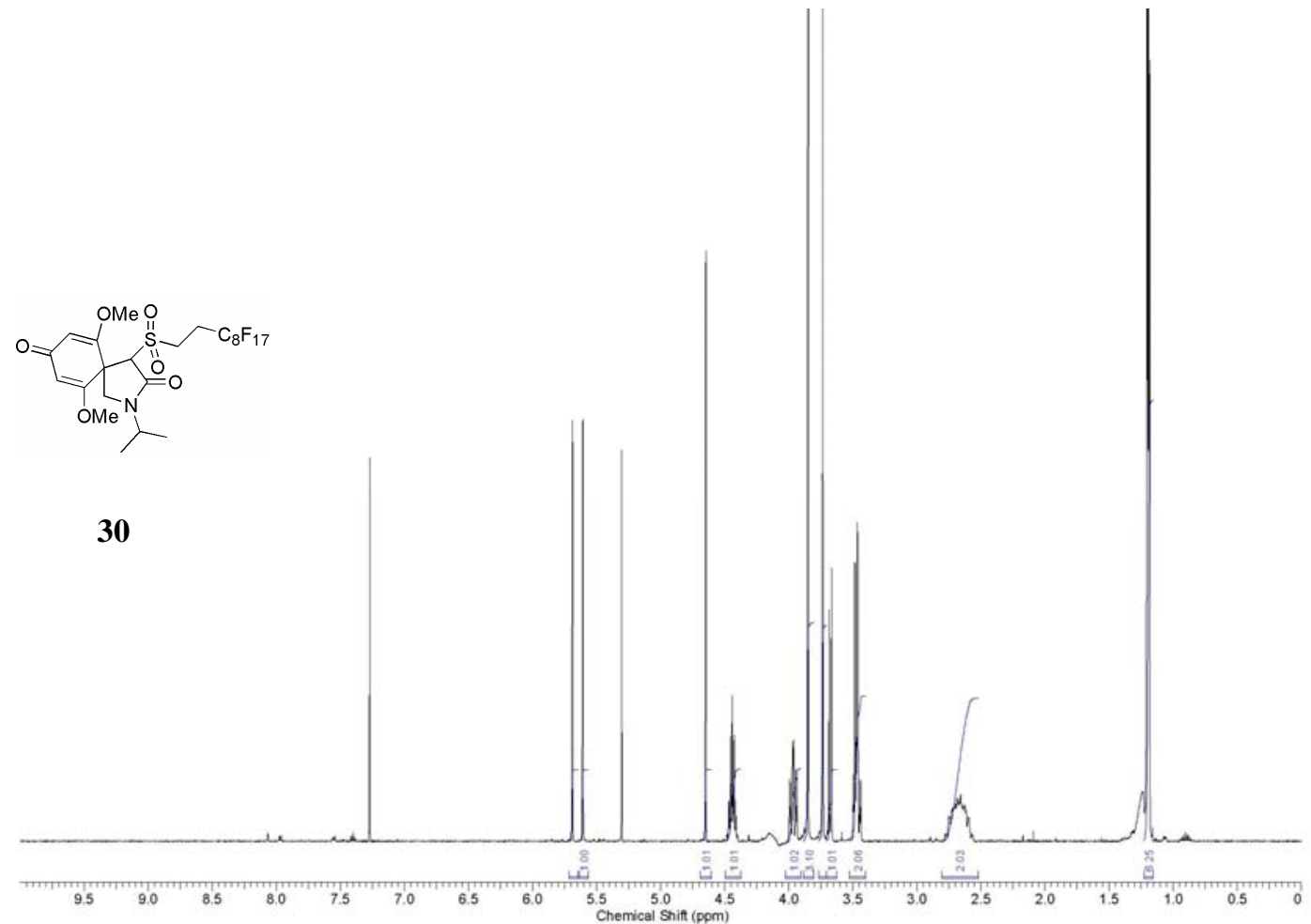

$125 \mathrm{MHz}\left(\mathrm{CDCl}_{3}\right)$

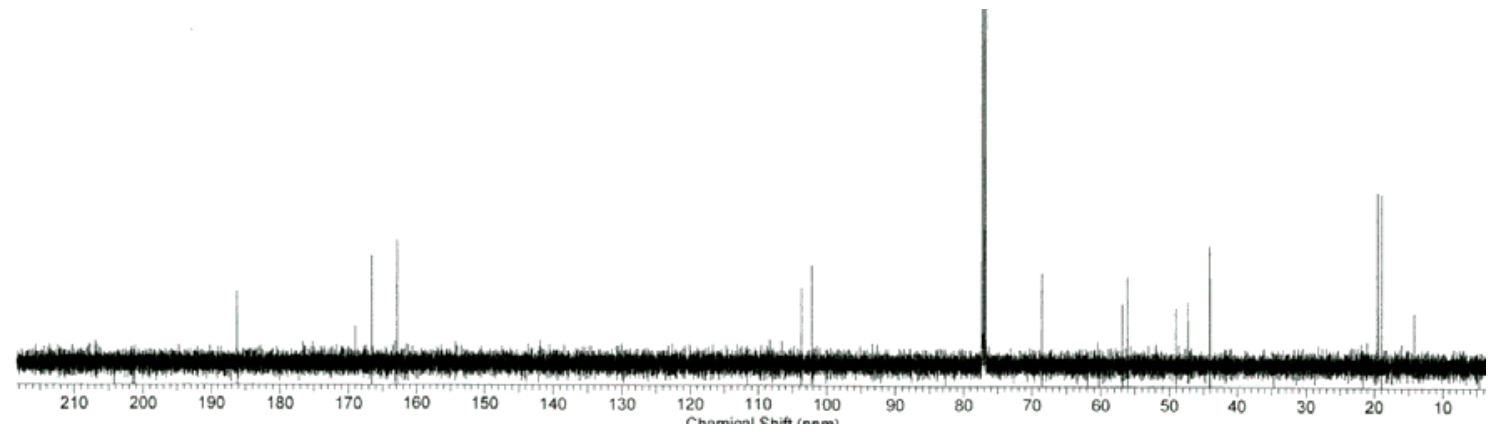


$500 \mathrm{MHz}\left(\mathrm{CDCl}_{3}\right)$

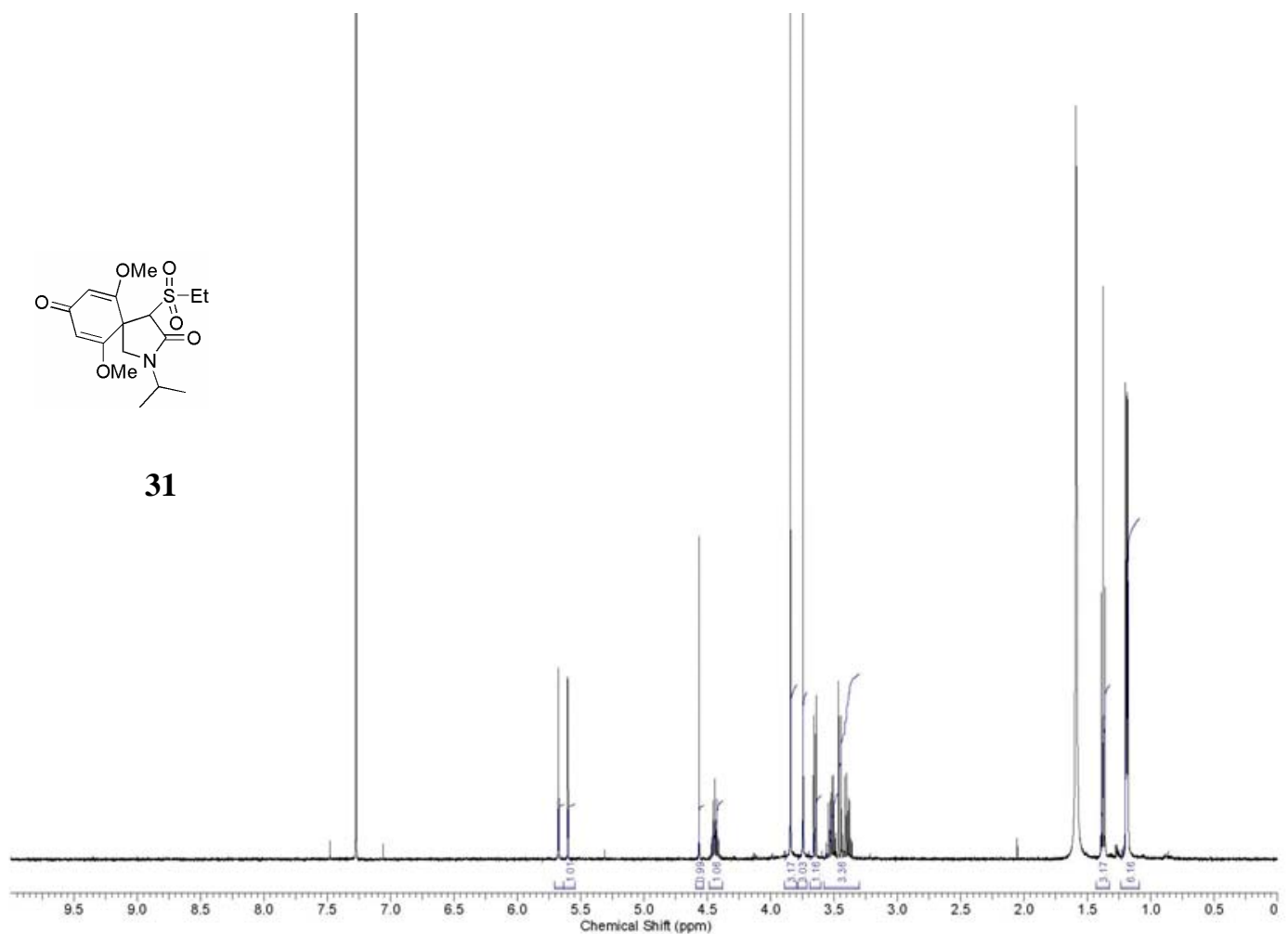

$125 \mathrm{MHz}\left(\mathrm{CDCl}_{3}\right)$

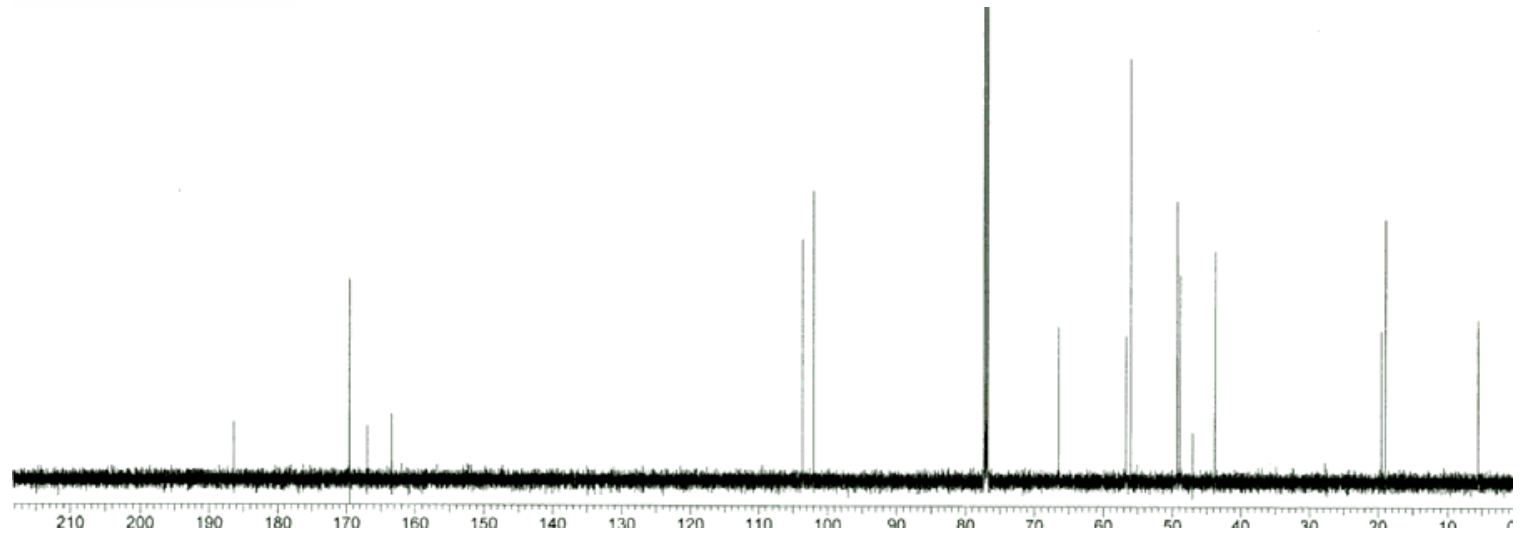


$500 \mathrm{MHz}\left(\mathrm{CDCl}_{3}\right)$

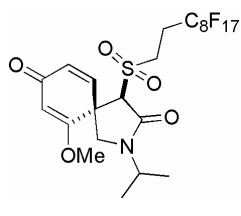

32

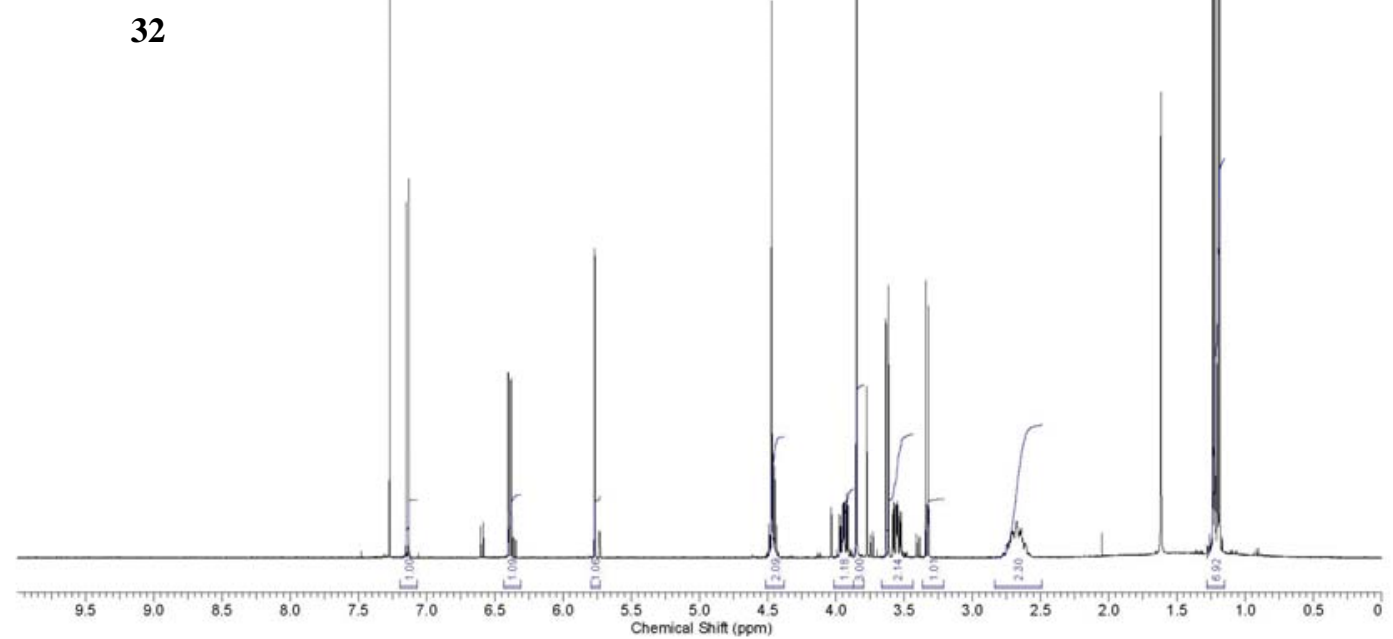

$125 \mathrm{MHz}\left(\mathrm{CDCl}_{3}\right)$

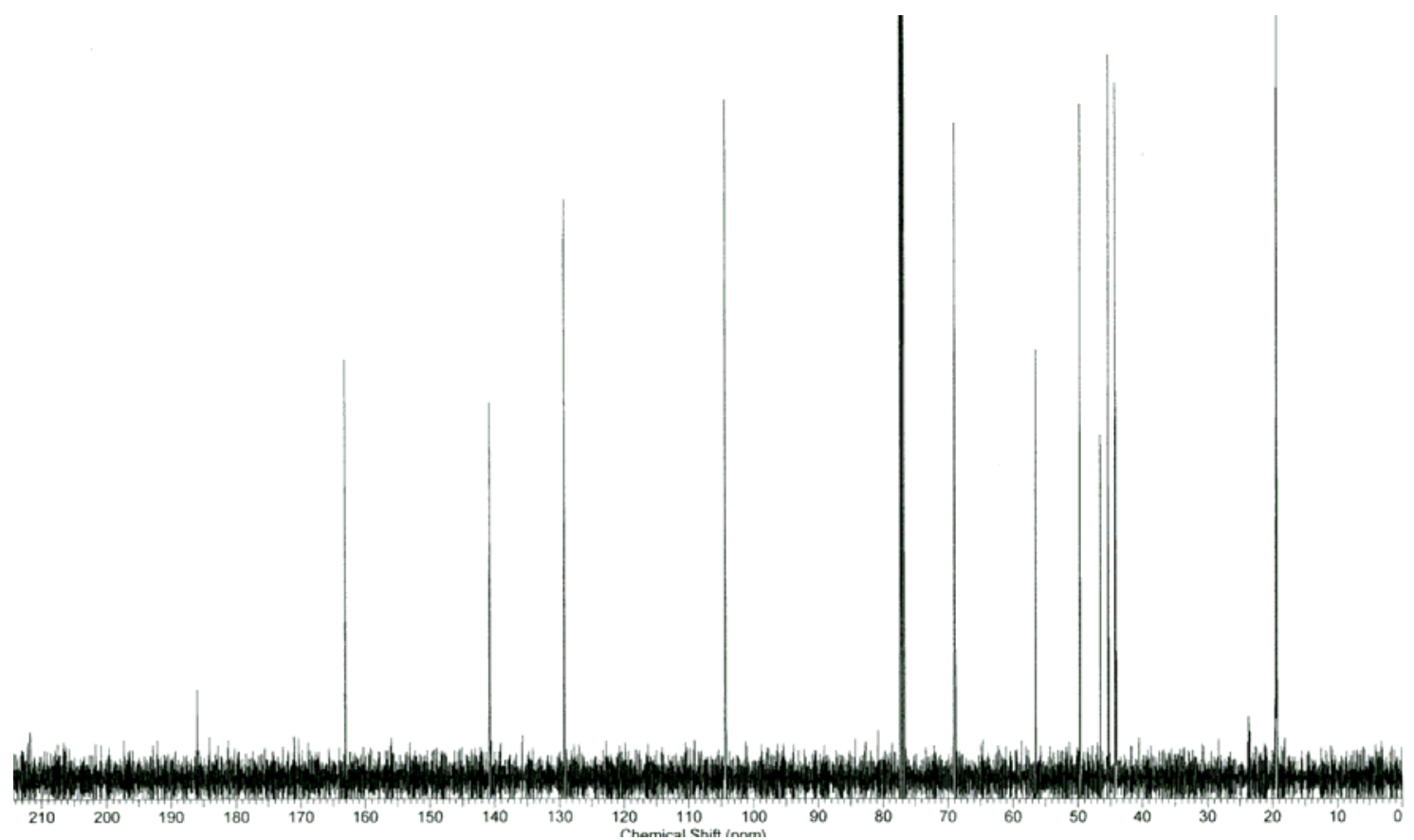


$500 \mathrm{MHz}\left(\mathrm{CDCl}_{3}\right)$

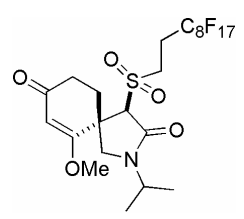

33

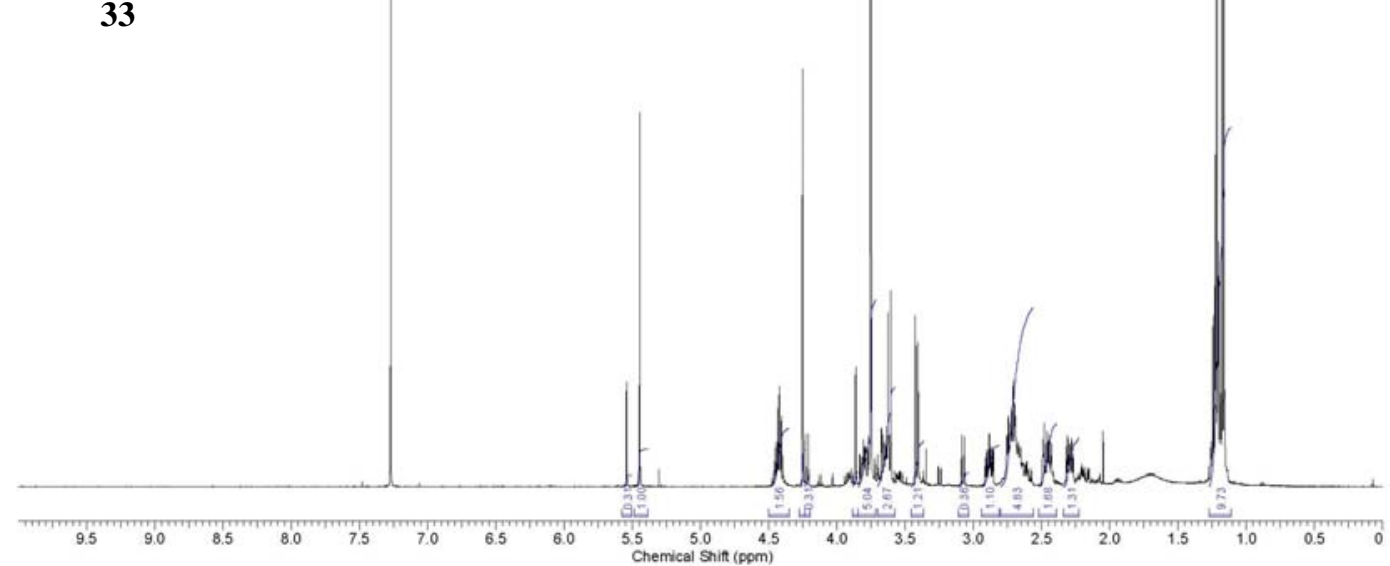

$125 \mathrm{MHz}\left(\mathrm{CDCl}_{3}\right)$

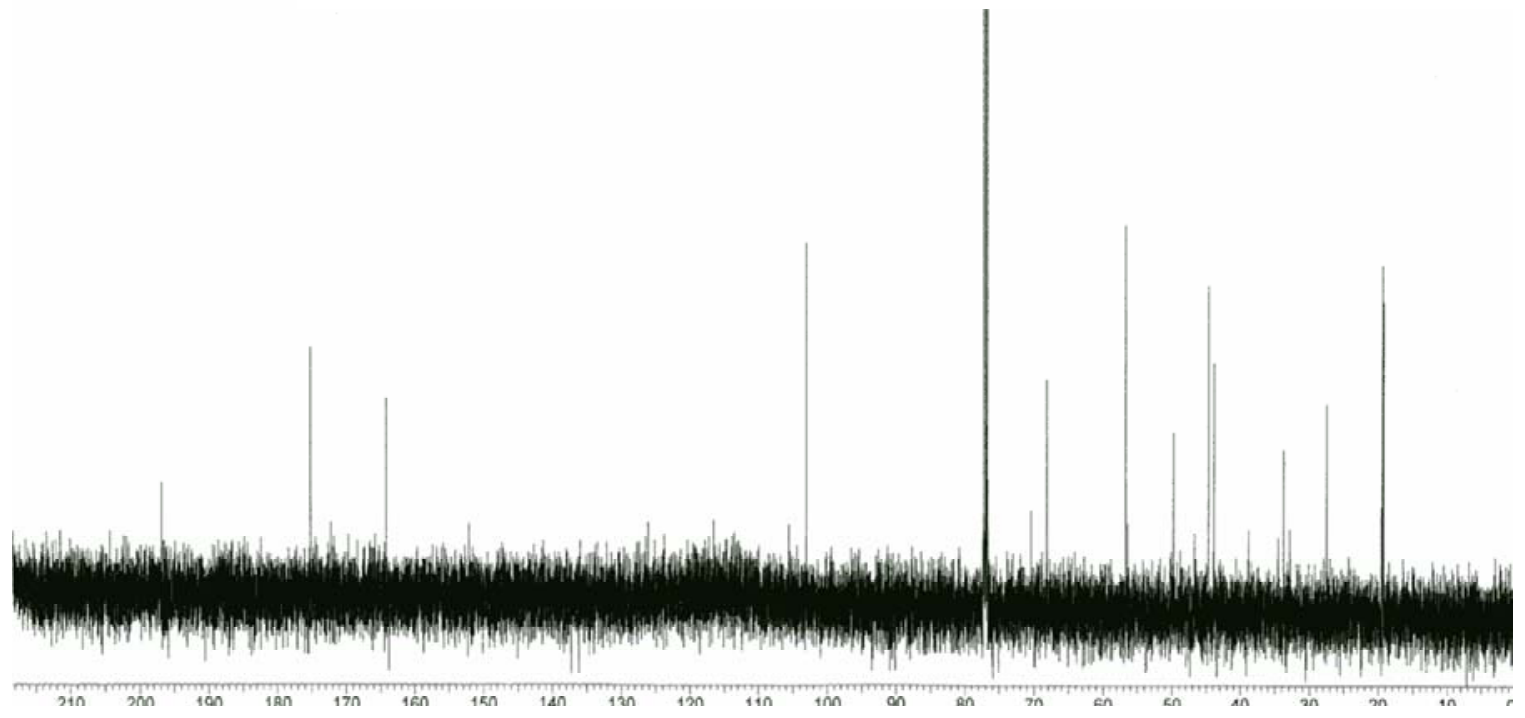


$500 \mathrm{MHz}\left(\mathrm{CDCl}_{3}\right)$

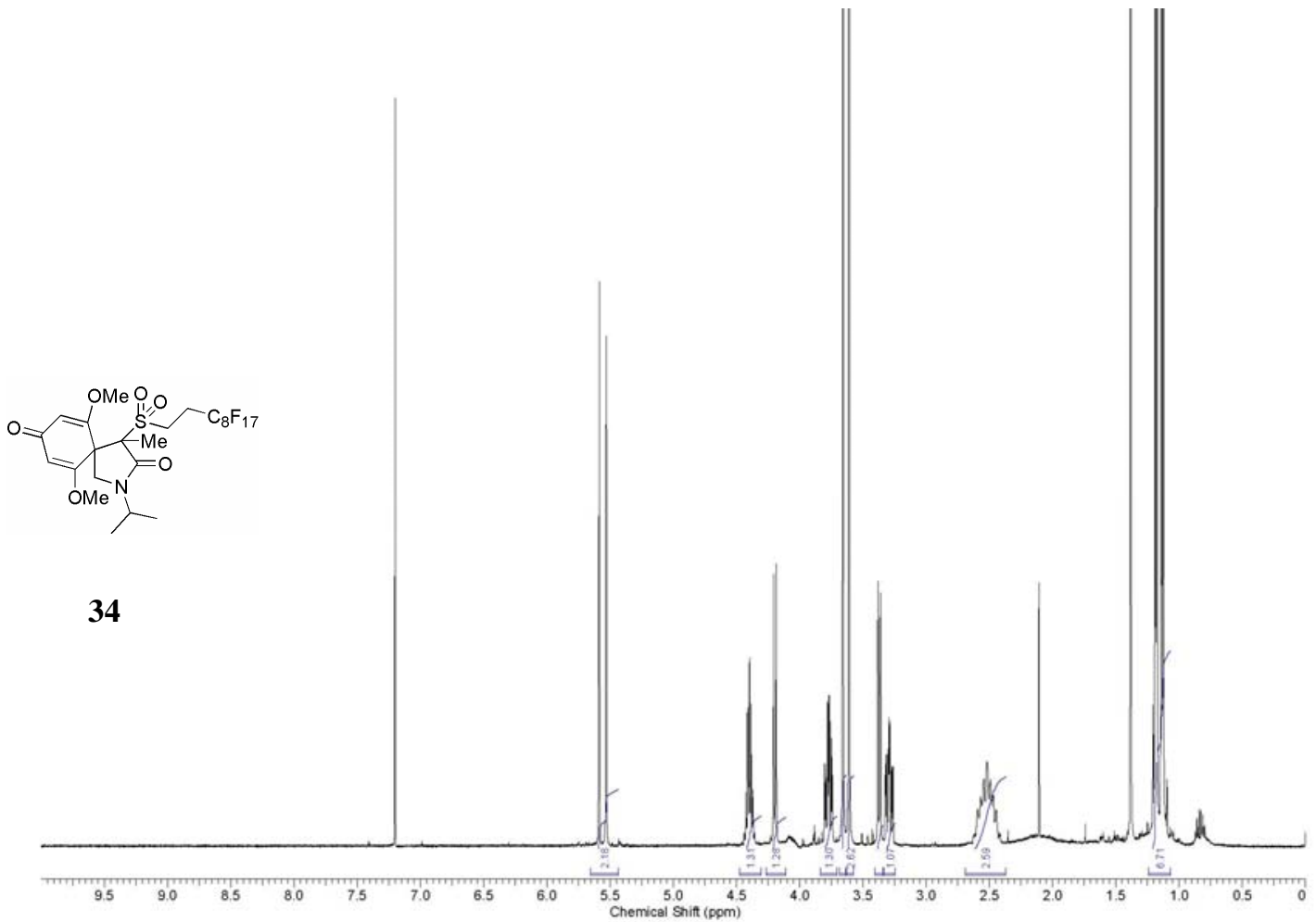

$125 \mathrm{MHz}\left(\mathrm{CDCl}_{3}\right)$

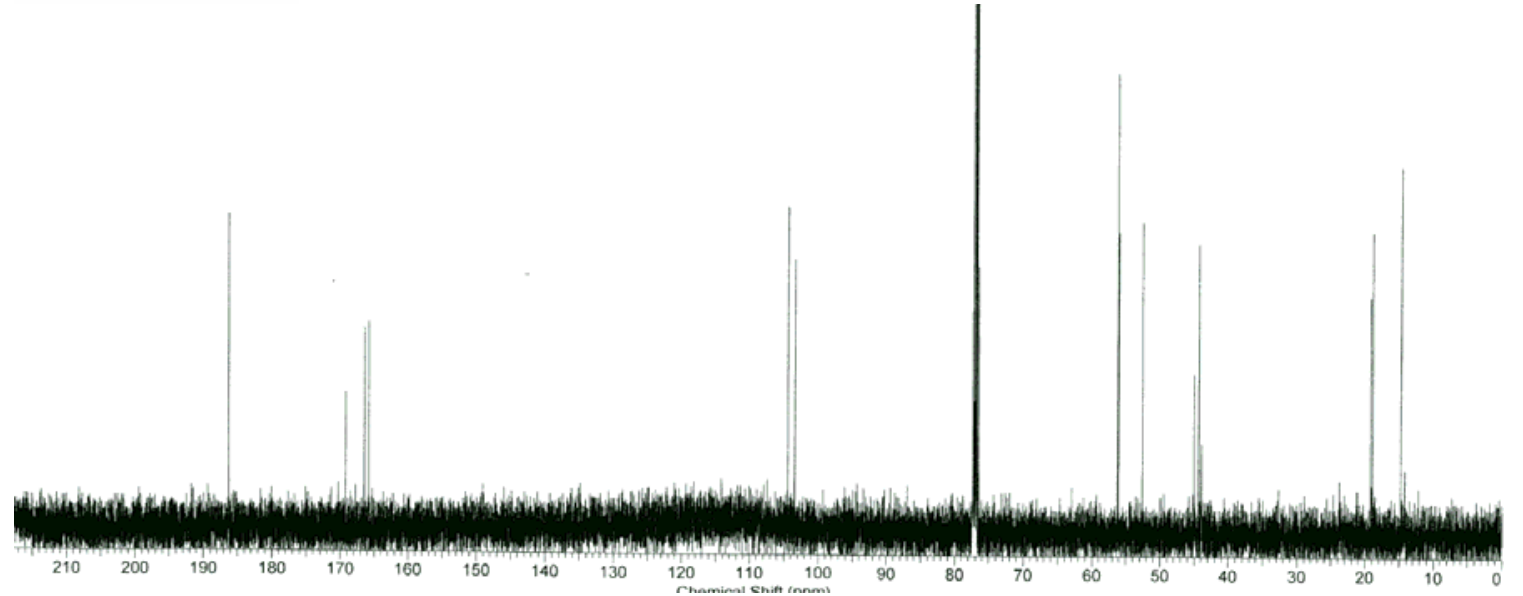


$300 \mathrm{MHz}\left(\mathrm{CDCl}_{3}\right)$

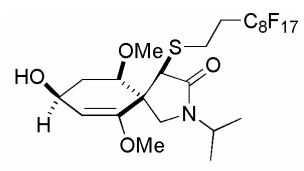

36

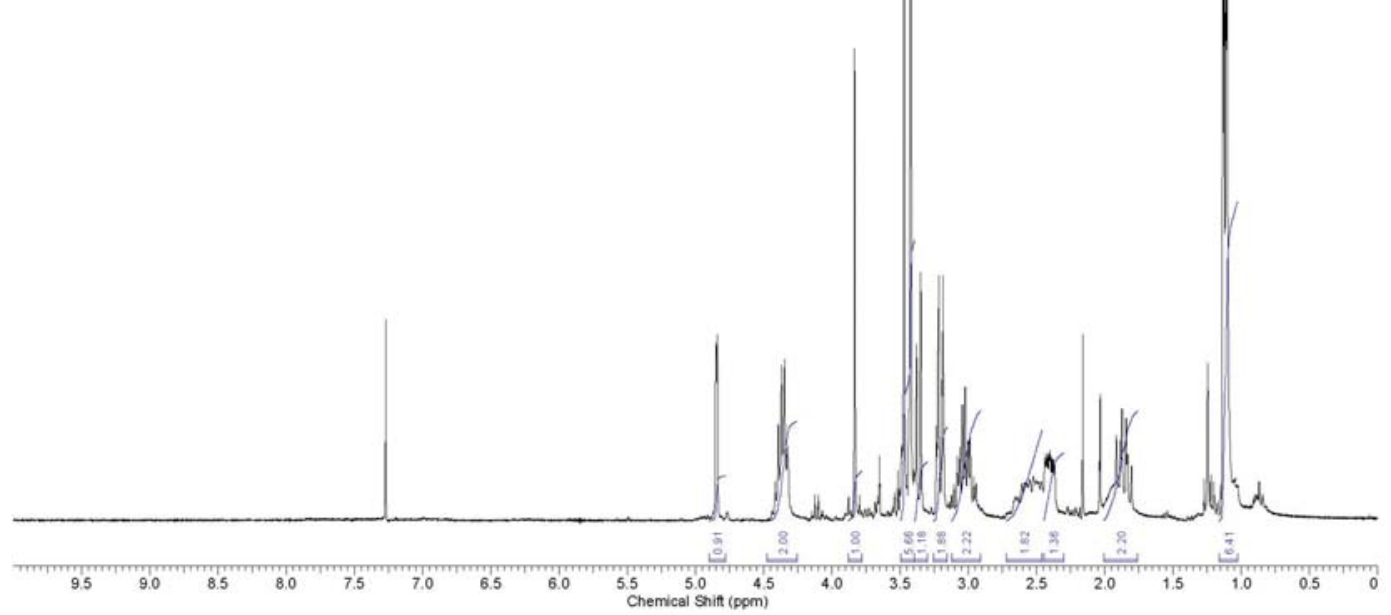

$75 \mathrm{MHz}\left(\mathrm{CDCl}_{3}\right)$

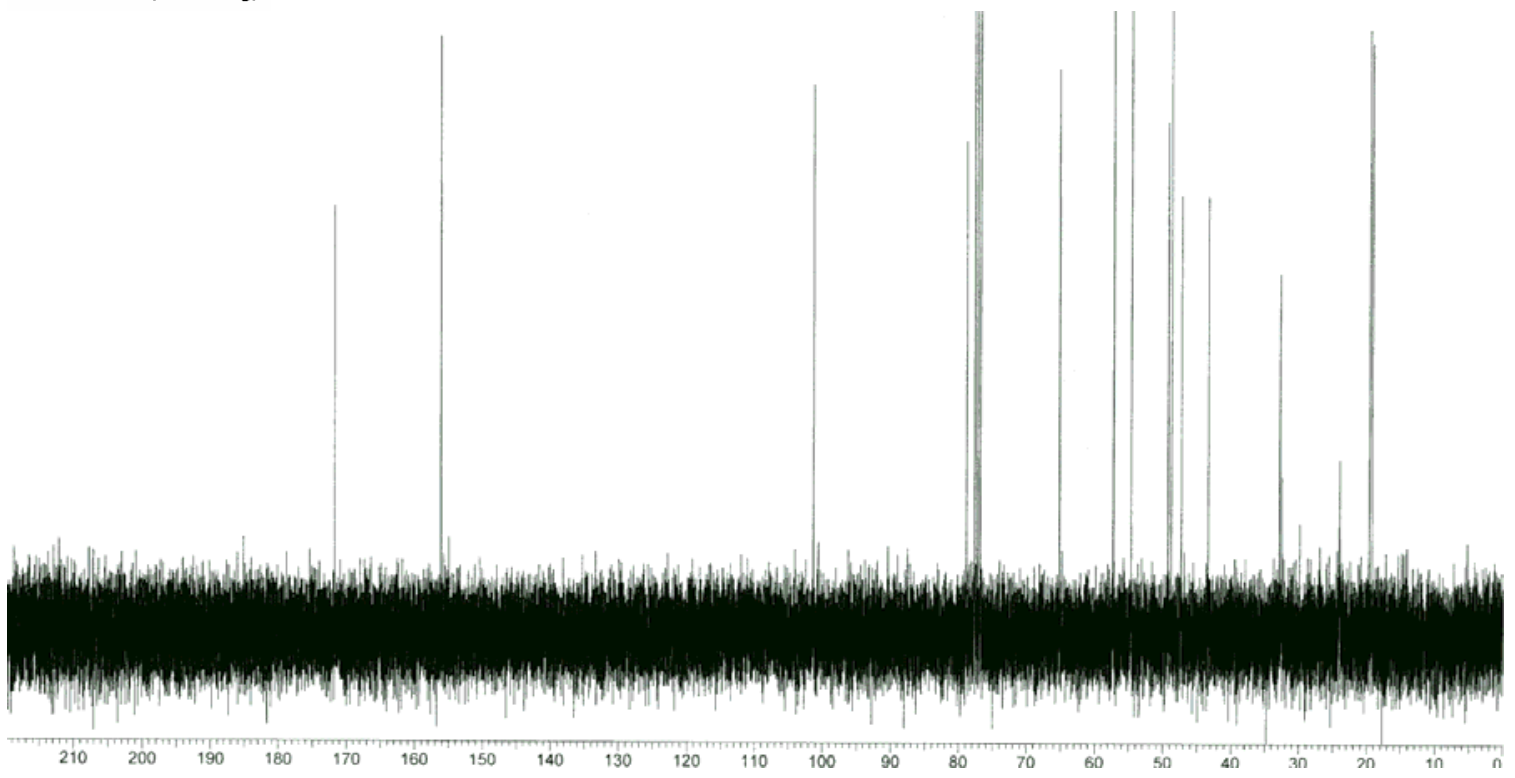


$500 \mathrm{MHz}\left(\mathrm{CDCl}_{3}\right)$

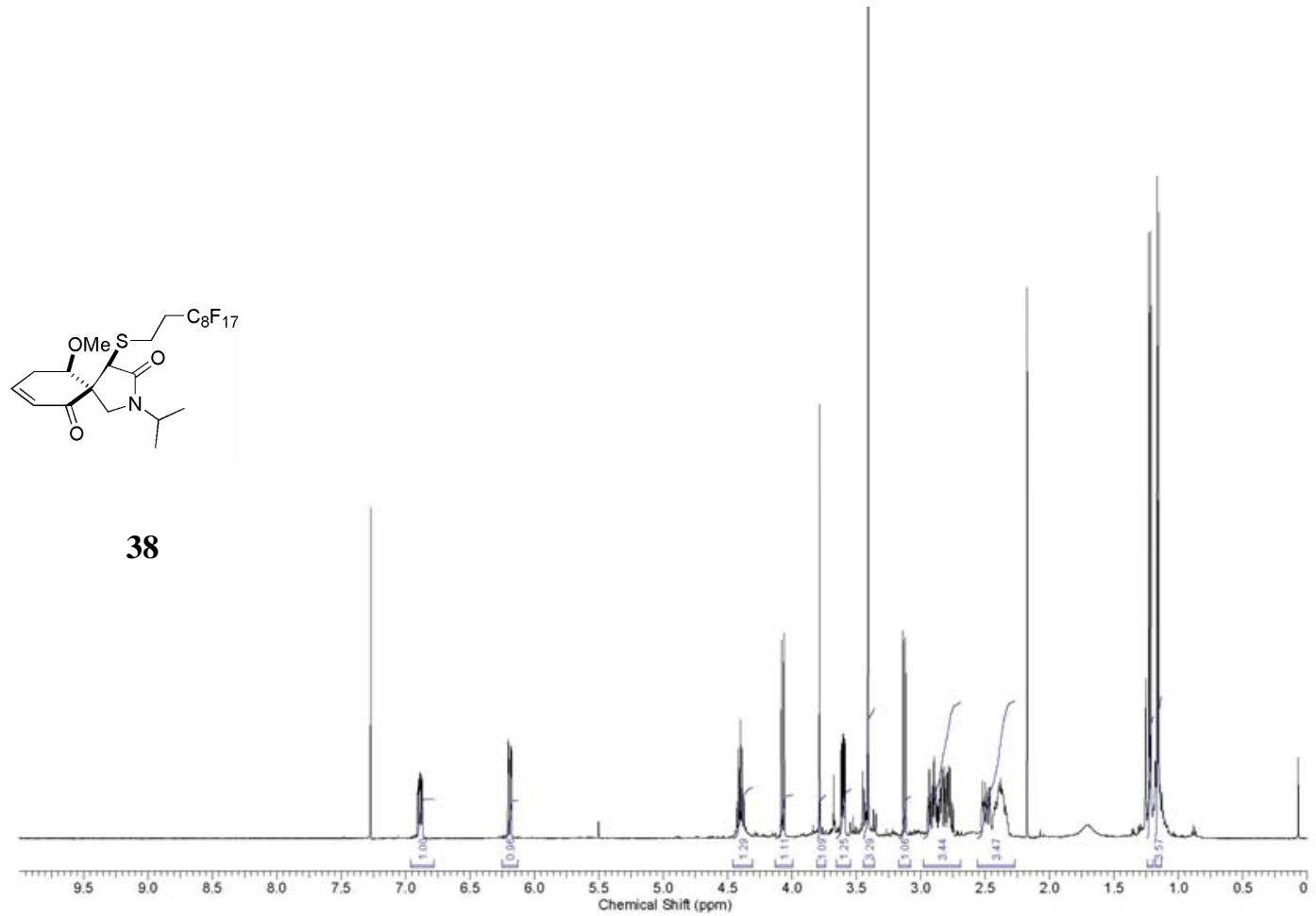

$125 \mathrm{MHz}\left(\mathrm{CDCl}_{3}\right)$

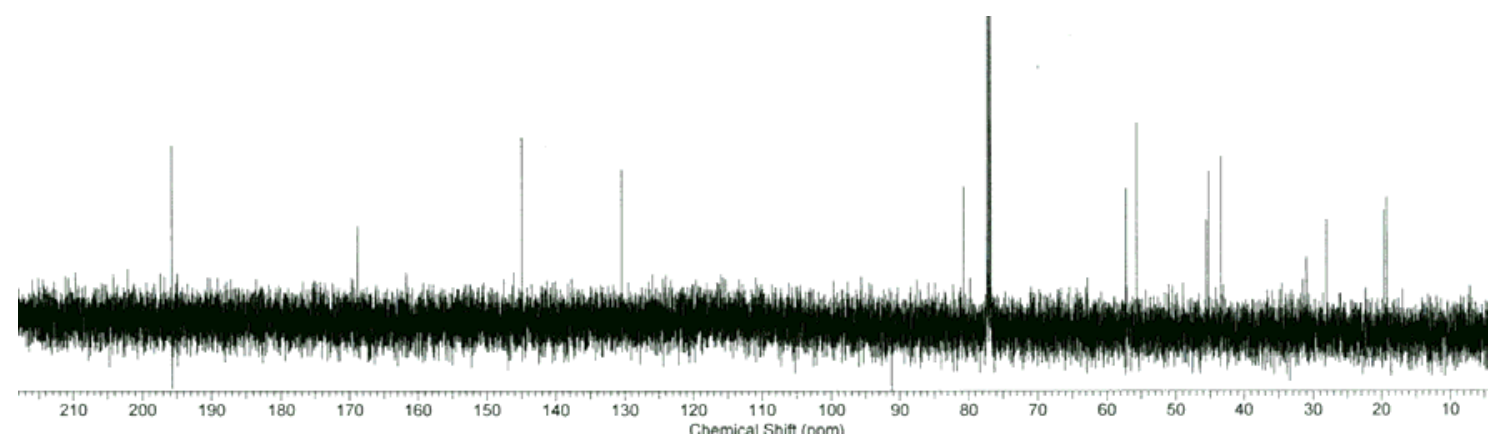


$500 \mathrm{MHz}\left(\mathrm{CDCl}_{3}\right)$

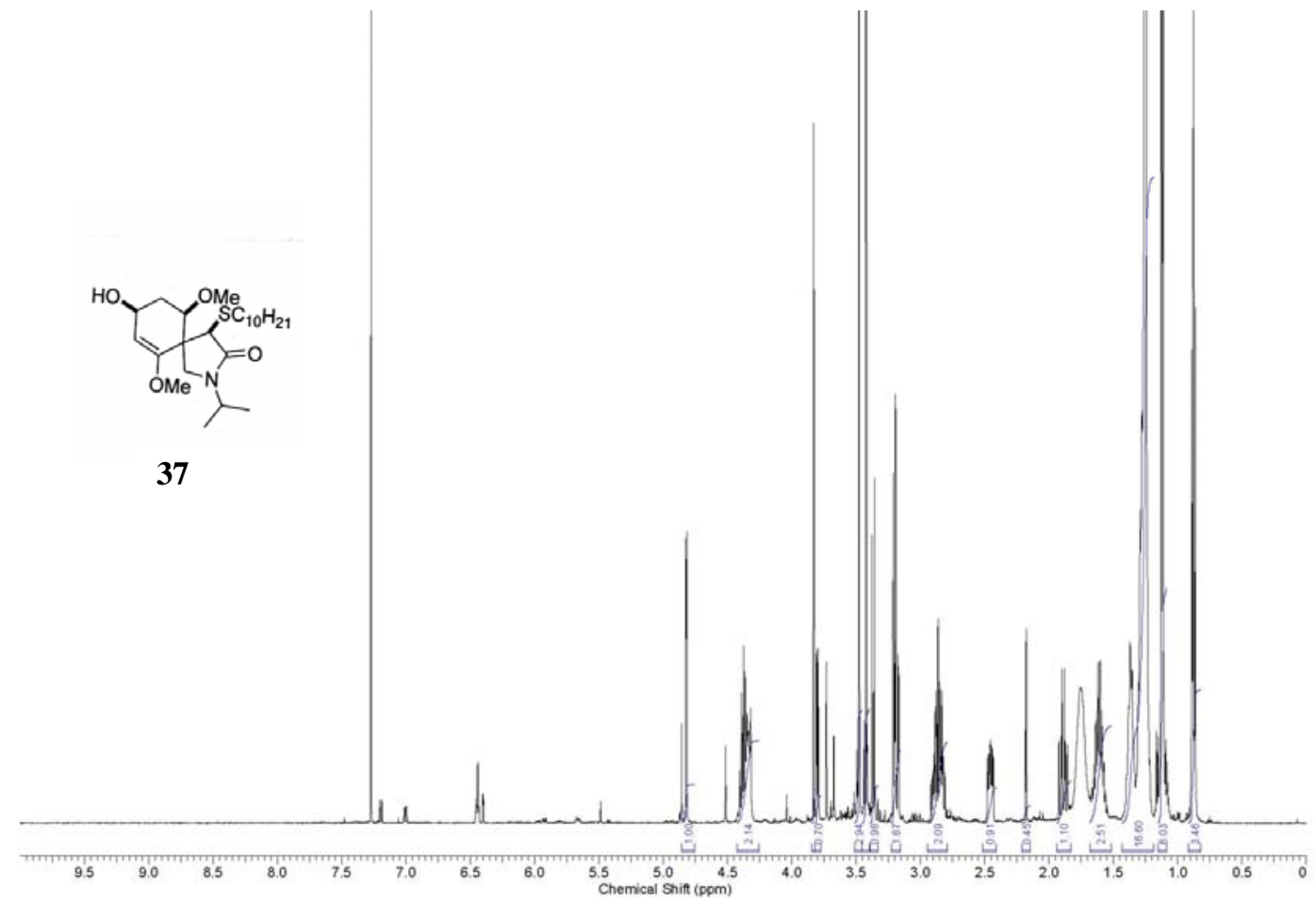

$125 \mathrm{MHz}\left(\mathrm{CDCl}_{3}\right)$

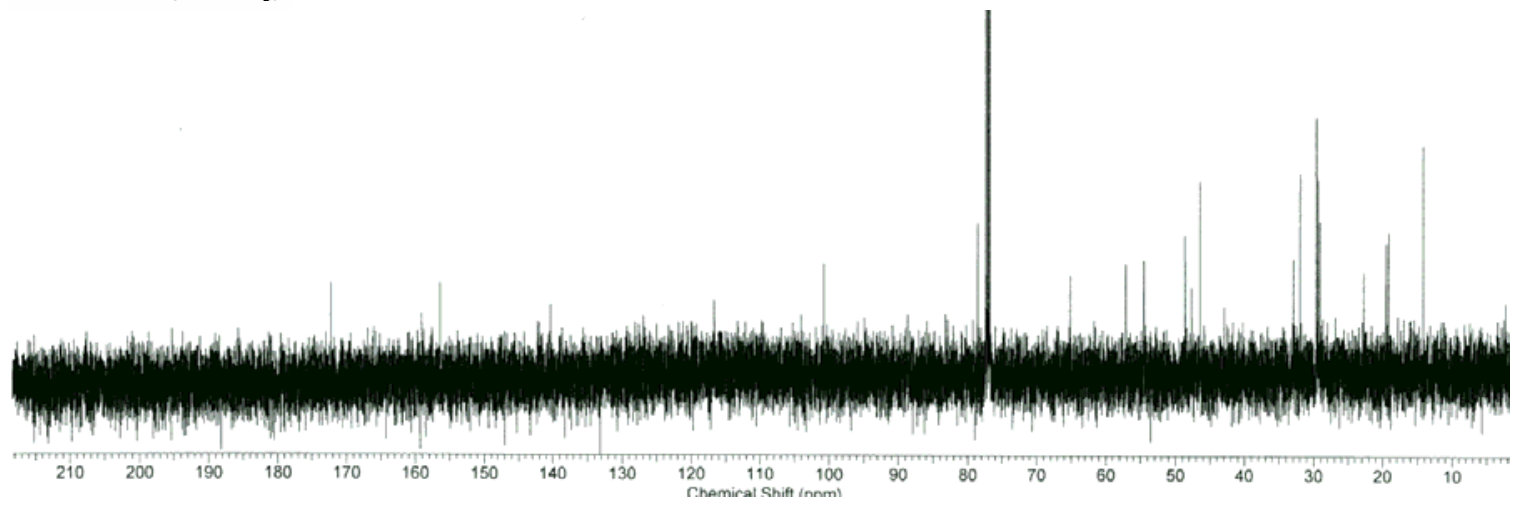


X-Ray crystal structure of $\mathbf{1 0}$

CCDC 669889

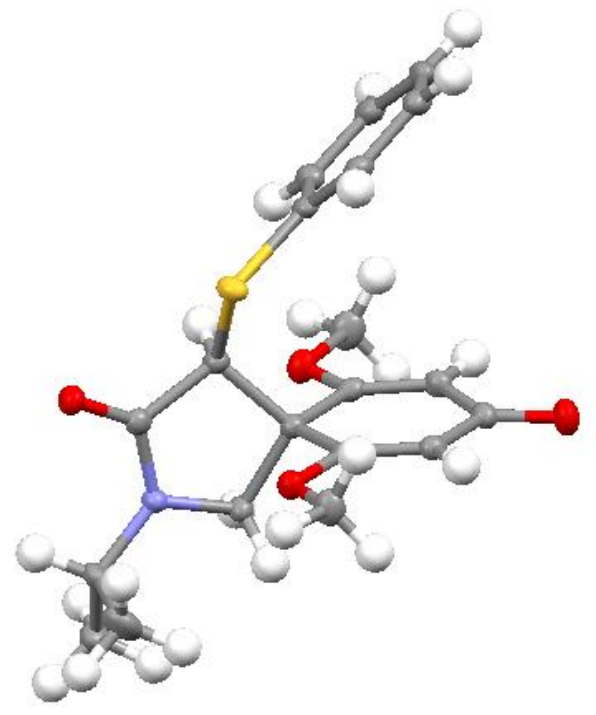

X-Ray crystal structure of 31

CCDC 669888

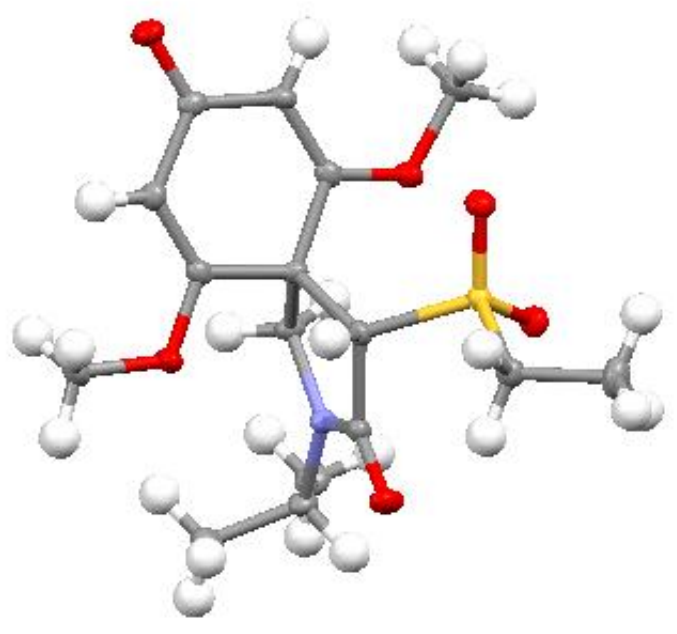

